\title{
Cochrane
}

Library

Cochrane Database of Systematic Reviews

\section{Selective noradrenaline reuptake inhibitors for schizophrenia} (Review)

Matthews PRL, Horder J, Pearce M

Matthews PRL, Horder J, Pearce M.

Selective noradrenaline reuptake inhibitors for schizophrenia.

Cochrane Database of Systematic Reviews 2018, Issue 1. Art. No.: CD010219.

DOI: 10.1002/14651858.CD010219.pub2.

www.cochranelibrary.com 
TABLE OF CONTENTS

ABSTRACT

PLAIN LANGUAGE SUMMARY

SUMMARY OF FINDINGS

BACKGROUND

OBJECTIVES

METHODS

RESULTS

Figure 1.

Figure 2.

Figure 3.

DISCUSSION

AUTHORS' CONCLUSIONS

ACKNOWLEDGEMENTS

REFERENCES

CHARACTERISTICS OF STUDIES

DATA AND ANALYSES

Analysis 1.1. Comparison 1 SELECTIVE NORADRENALINE REUPTAKE INHIBITORS versus PLACEBO, Outcome 1 Primary outcome

- A. Mental state: Specific - clinically significant response or improvement in negative symptoms (SANS, high=worse) - short term (12 weeks).

Analysis 1.2. Comparison 1 SELECTIVE NORADRENALINE REUPTAKE INHIBITORS versus PLACEBO, Outcome 2 Primary outcome - B. Clinical global response: Clinically significant response or improvement in global status (CGI-S, high=worse) - short term (4 weeks).

Analysis 1.3. Comparison 1 SELECTIVE NORADRENALINE REUPTAKE INHIBITORS versus PLACEBO, Outcome 3 Mental state: 1. General - clinically significant response or improvement in general/overall symptoms as defined in each study - short term (2-12 weeks)

Analysis 1.4. Comparison 1 SELECTIVE NORADRENALINE REUPTAKE INHIBITORS versus PLACEBO, Outcome 4 Mental state: $2 a$. General: Average general/overall symptoms score (various scales, high=worse) - short term (2 - 12 weeks).

Analysis 1.5. Comparison 1 SELECTIVE NORADRENALINE REUPTAKE INHIBITORS versus PLACEBO, Outcome 5 Mental state: $2 \mathrm{~b}$. General: Average general/overall symptoms score (various scales, high=worse) - medium term (13 - 26 weeks).

Analysis 1.6. Comparison 1 SELECTIVE NORADRENALINE REUPTAKE INHIBITORS versus PLACEBO, Outcome 6 Mental state: $3 a$. Specific: Average symptoms score (various scales, high=worse) - short term (2 - 12 weeks).

Analysis 1.7. Comparison 1 SELECTIVE NORADRENALINE REUPTAKE INHIBITORS versus PLACEBO, Outcome 7 Mental state: $3 \mathrm{~b}$. Specific: Average symptoms score (various scales, high=worse) - medium term (13 - 26 weeks).

Analysis 1.8. Comparison 1 SELECTIVE NORADRENALINE REUPTAKE INHIBITORS versus PLACEBO, Outcome 8 Mental state: $4 a$. General and specific: Average score (various scales, high=worse) - skewed results - short term (2 - 12 weeks).

Analysis 1.9. Comparison 1 SELECTIVE NORADRENALINE REUPTAKE INHIBITORS versus PLACEBO, Outcome 9 Mental state: $4 \mathrm{~b}$. General and specific: Average score (various scales, high=worse) - skewed results - medium term (13 - 26 weeks).

Analysis 1.10. Comparison 1 SELECTIVE NORADRENALINE REUPTAKE INHIBITORS versus PLACEBO, Outcome 10 Cognitive functioning: 1. General - average composite cognitive functioning score (SMD).

Analysis 1.11. Comparison 1 SELECTIVE NORADRENALINE REUPTAKE INHIBITORS versus PLACEBO, Outcome 11 Cognitive functioning: 2. Specific - average individual scores (SMD) - short term (2 - 12 weeks).

Analysis 1.12. Comparison 1 SELECTIVE NORADRENALINE REUPTAKE INHIBITORS versus PLACEBO, Outcome 12 Quality of life: Average quality of life score (GQOLI-74, high=better) - short term (2 - 12 weeks).

Analysis 1.13. Comparison 1 SELECTIVE NORADRENALINE REUPTAKE INHIBITORS versus PLACEBO, Outcome 13 Clinical global response: 1a. Average clinical global status score (CGI-S, high=worse) - short term (2 - 12 weeks).

Analysis 1.14. Comparison 1 SELECTIVE NORADRENALINE REUPTAKE INHIBITORS versus PLACEBO, Outcome 14 Clinical global response: 1 b. Average clinical global status score (various scales, high=worse) - short term (2 - 12 weeks) - skewed results. .... Analysis 1.15. Comparison 1 SELECTIVE NORADRENALINE REUPTAKE INHIBITORS versus PLACEBO, Outcome 15 Leaving the study early: 1a. Short term (2 - 12 weeks).

Analysis 1.16. Comparison 1 SELECTIVE NORADRENALINE REUPTAKE INHIBITORS versus PLACEBO, Outcome 16 Leaving the study early: 1b. Medium term (13 - 26 weeks).

Analysis 1.17. Comparison 1 SELECTIVE NORADRENALINE REUPTAKE INHIBITORS versus PLACEBO, Outcome 17 Adverse effects: 1. General - short term (binary, 2 - 12 weeks). 
Analysis 1.18. Comparison 1 SELECTIVE NORADRENALINE REUPTAKE INHIBITORS versus PLACEBO, Outcome 18 Adverse effects: 2a.i. Specific - short term (binary, 2 - 12 weeks).

Analysis 1.19. Comparison 1 SELECTIVE NORADRENALINE REUPTAKE INHIBITORS versus PLACEBO, Outcome 19 Adverse effects: 2a.ii. Specific - extrapyramidal - average change score (continuous, various scales, high=worse) - short term ( 2 - 12 weeks). .... Analysis 1.20. Comparison 1 SELECTIVE NORADRENALINE REUPTAKE INHIBITORS versus PLACEBO, Outcome 20 Adverse effects: 2a.iii. Specific - extrapyramidal - average change score (continuous, various scales, high=worse) - short term (2 - 12 weeks) skewed results.

Analysis 1.21. Comparison 1 SELECTIVE NORADRENALINE REUPTAKE INHIBITORS versus PLACEBO, Outcome 21 Adverse effects: 2b.i. Specific - medium term (binary, 13 - 26 weeks).

Analysis 1.22. Comparison 1 SELECTIVE NORADRENALINE REUPTAKE INHIBITORS versus PLACEBO, Outcome 22 Adverse effects: 2a.iv. Specific - metabolic - average weight gain (continuous, increase in $\mathrm{kg}$ ).

Analysis 1.23. Comparison 1 SELECTIVE NORADRENALINE REUPTAKE INHIBITORS versus PLACEBO, Outcome 23 Social or general functioning: Average social functioning score (various subscales) - short-term (2 - 12 weeks).

Analysis 2.1. Comparison 2 SELECTIVE NORADRENALINE REUPTAKE INHIBITORS versus ACTIVE CONTROL, Outcome 1 Mental state: 1a. General: Average general/overall symptoms score (PANSS general, high=worse) - short term (2 - 12 weeks).

Analysis 2.2. Comparison 2 SELECTIVE NORADRENALINE REUPTAKE INHIBITORS versus ACTIVE CONTROL, Outcome 2 Mental state: 1b. General: Average general/overall symptoms score (various scales, high=worse) - medium term (13 - 26 weeks).

Analysis 2.3. Comparison 2 SELECTIVE NORADRENALINE REUPTAKE INHIBITORS versus ACTIVE CONTROL, Outcome 3 Mental state: 2a. Specific: Average symptoms score (various scales, high=worse) - short term (2 - 12 weeks).

Analysis 2.4. Comparison 2 SELECTIVE NORADRENALINE REUPTAKE INHIBITORS versus ACTIVE CONTROL, Outcome 4 Mental state: 2b. Specific: Average symptoms score (various scales, high=worse) - medium term (13 - 26 weeks).

Analysis 2.5. Comparison 2 SELECTIVE NORADRENALINE REUPTAKE INHIBITORS versus ACTIVE CONTROL, Outcome 5 Mental state: 3a. General and specific: Average score (various scales, high=worse) - skewed results - short term (2 - 12 weeks).

Analysis 2.6. Comparison 2 SELECTIVE NORADRENALINE REUPTAKE INHIBITORS versus ACTIVE CONTROL, Outcome 6 Mental state: 3b. General and specific: Average score (various scales, high=worse) - skewed results - medium term (13 - 26 weeks). .... Analysis 2.7. Comparison 2 SELECTIVE NORADRENALINE REUPTAKE INHIBITORS versus ACTIVE CONTROL, Outcome 7 Clinical global response: Average clinical global status score (CGI-S, high=worse) - short term (2 - 12 weeks).

Analysis 2.8. Comparison 2 SELECTIVE NORADRENALINE REUPTAKE INHIBITORS versus ACTIVE CONTROL, Outcome 8 Leaving the study early: $1 \mathrm{a}$. Short term (2 - 12 weeks).

Analysis 2.9. Comparison 2 SELECTIVE NORADRENALINE REUPTAKE INHIBITORS versus ACTIVE CONTROL, Outcome 9 Leaving the study early: $1 \mathrm{~b}$. Medium term (13 - 26 weeks).

ADDITIONAL TABLES

APPENDICES

HISTORY

CONTRIBUTIONS OF AUTHORS 
[Intervention Review]

\section{Selective noradrenaline reuptake inhibitors for schizophrenia}

Paul R L Matthews ${ }^{1}$, Jamie Horder ${ }^{2}$, Michael Pearce ${ }^{3}$

1North Kildare Mental Health Service, Kildare West Wicklow MHS, Celbridge, Ireland. ${ }^{2}$ Department of Forensic and Neurodevelopmental Sciences, King's College London, London, UK. ${ }^{3}$ Department of General and Older Adult Psychiatry, Oxford Health NHS Foundation Trust, Oxford, UK

Contact: Paul R L Matthews, North Kildare Mental Health Service, Kildare West Wicklow MHS, Celbridge Community Health Centre, Shackleton Road, Celbridge, Co. Kildare, Ireland. paul.matthews@hse.ie.

Editorial group: Cochrane Schizophrenia Group.

Publication status and date: New, published in Issue 1, 2018.

Citation: Matthews PRL, Horder J, Pearce M. Selective noradrenaline reuptake inhibitors for schizophrenia. Cochrane Database of Systematic Reviews 2018, Issue 1. Art. No.: CD010219. DOI: 10.1002/14651858.CD010219.pub2.

Copyright ( 2018 The Cochrane Collaboration. Published by John Wiley \& Sons, Ltd.

\section{A B S T R A C T}

\section{Background}

Schizophrenia is frequently a chronic and disabling illness with a heterogeneous range of symptoms. The positive symptoms usually respond to antipsychotics but the cognitive and negative symptoms of schizophrenia are difficult to treat with conventional antipsychotics and significantly impact on quality of life and social outcomes. Selective noradrenaline reuptake inhibitors (NRIs) increase prefrontal dopamine and noradrenaline levels without significantly affecting subcortical dopamine levels, making them an attractive candidate for treating cognitive and negative symptoms.

\section{Objectives}

To investigate the effects of selective noradrenaline reuptake inhibitors (NRIs), compared with a placebo or control treatment, for people with schizophrenia.

\section{Search methods}

We searched the Cochrane Schizophrenia Group's Trials Register (up to 7 February 2017) which is based on regular searches of MEDLINE, Embase, CINAHL, BIOSIS, AMED, PubMed, PsycINFO, and registries of clinical trials. There are no language, date, document type, or publication status limitation for inclusion of records into the register. We inspected references of all included studies for further relevant studies.

\section{Selection criteria}

We included randomised controlled trials (RCTs) comparing NRIs with either a control treatment or placebo for people with schizophrenia or related disorders (such as schizoaffective disorder) by any means of diagnosis. We included trials that met our selection criteria and provided useable information.

\section{Data collection and analysis}

We independently inspected all citations from searches, identified relevant abstracts, and independently extracted data from all included studies. For binary data we calculated risk ratio (RR), for continuous data we calculated mean difference (MD), and for cognitive outcomes we derived standardised mean difference (SMD) effect sizes, all with 95\% confidence intervals (CI) and using a random-effects model. We assessed risk of bias for the included studies and used the GRADE approach to produce a 'Summary of findings' table which included our prespecified main outcomes of interest. 


\section{Main results}

Searching identified 113 records. We obtained the full text of 48 of these records for closer inspection. Sixteen trials, randomising a total of 919 participants are included. The majority of trials included adults with schizophrenia or similar illness who were inpatients, and while they were poorly characterised, most appeared to include patients with a chronic presentation. The intervention NRI in nine of the 16 trials was reboxetine, with atomoxetine and viloxazine used in the remaining trials. 14 trials compared NRIs with placebo. Only two trials provided data to compare NRIs against an active control and both compared reboxetine to citalopram but at 4 weeks and 24 weeks respectively so they could not be combined in a meta-analysis.

One trial was described as 'open' and we considered it to be at high risk of bias for randomisation and blinding, three trials were at high risk of bias for attrition, six for reporting, and two for other sources of bias. Our main outcomes of interest were significant response or improvement in positive/negative mental state, global state and cognitive functioning, average cognitive functioning scores, significant response or improvement in quality of life and incidence of nausea. All data for main outcomes were short term.

\section{NRIs versus placebo}

Mental state results showed significantly greater rates of improvement in negative symptoms scores $(1 \mathrm{RCT}, \mathrm{n}=50 ; \mathrm{RR} 3.17,95 \% \mathrm{Cl} 1.52$ to 6.58; very low quality evidence) with NRIs on the PANSS negative. No data were reported for significant response or improvement in positive symptoms, but average endpoint PANSS positive scores were available and showed no difference between NRIs and placebo (5 RCTs, $n=294 ; M D-0.16,95 \% \mathrm{Cl}-0.96$ to 0.63 ; low-quality evidence). Improvement in clinical global status was similar between groups (1 $\mathrm{RCT}, \mathrm{n}=28$; RR $0.99,95 \% \mathrm{Cl} 0.45$ to 2.20 ; very low quality evidence). Significant response or improvement in cognitive functioning data were not reported. Average composite cognitive scores showed no difference between NRIs and placebo (4 RCTs, $n=180 ;$ SMD 0.04, 95\% $\mathrm{Cl}-0.28$ to 0.36 ; low-quality evidence). Significant response or improvement in quality of life data were not reported, however average endpoint scores from the GQOLI-74 were reported. Those receiving NRIs had better quality of life scores compared to placebo $(1 \mathrm{RCT}, \mathrm{n}=$ 114; MD 9.36, $95 \% \mathrm{Cl} 7.89$ to 10.83; very low quality evidence). All-cause withdrawals did not differ between the treatment groups (8 RCTs, $\mathrm{n}=401$, RR $0.9495 \% \mathrm{Cl} 0.63$ to 1.39; moderate-quality evidence). Rates of nausea were not greater with NRIs (3 RCTs, $n=176 ;$ RR $0.49,95 \%$ $\mathrm{Cl} 0.10$ to 2.41 ; low-quality evidence).

\section{Authors' conclusions}

Our results provide tentative very low quality evidence that compared to placebo, NRIs (specifically reboxetine) may have a benefit on the negative symptoms of schizophrenia. Limited evidence also suggests that NRIs have no effect on the positive symptoms of schizophrenia or cognitive functioning. NRIs appear generally well tolerated with no real differences in adverse effects such as nausea noted between NRIs and placebo. However, these results are based on short-term follow-up and are poor quality - there is need for more good-quality evidence. A large RCT of reboxetine over a longer period of time, focusing specifically on negative and cognitive symptoms as well as more detailed and comprehensive reporting of outcomes, including adverse events, is required.

\section{PLAIN LANGUAGE SUMMARY}

\section{Using selective noradrenaline reuptake inhibitors (NRIs) to treat schizophrenia}

\section{Review question}

Are selective noradrenaline reuptake inhibitors (NRIs) effective for treating the symptoms, particularly the negative symptoms, of schizophrenia?

\section{Background}

People with schizophrenia often have positive symptoms such as hearing voices (hallucinations), bizarre beliefs (delusions), or unclear thinking (formal thought disorder). These can be treated successfully with antipsychotic medication. People with schizophrenia also have negative symptoms such as social withdrawal or lack of motivation and cognitive symptoms such as difficulties making decisions and problems with attention or memory. Negative symptoms often are long term and reduce quality of life. Unlike the positive symptoms, there is a lack of effective medications to treat these negative symptoms.

Noradrenaline reuptake inhibitors (such as reboxetine or atomoxetine) are medicines that might help with the negative symptoms of schizophrenia in particular. There have been trials investigating the effectiveness of NRIs for people with schizophrenia but results found NRIs had little benefit. However, these were very small studies. We wanted to see whether combining results from all these trials would provide better-quality evidence.

\section{Searching and study characteristics}

The Information Specialist of Cochrane Schizophrenia searched their specialised register for relevant trials up to February 2017. We found sixteen trials that could be included. These trials randomised 919 adults with schizophrenia to receive either an NRI, a placebo (dummy treatment), or an antidepressant. All participants continued to receive the antipsychotic medications they were already taking. Most trials included participants who were in hospital and who had had symptoms of schizophrenia for a long time. 


\section{Key results and quality of the evidence available}

Our main areas of interest were the effect NRIs have on improving mental and global state, cognitive functioning and quality of life for people with schizophrenia; and if NRIs cause unpleasant side-effects such as nausea.

We found that compared to placebo treatment, NRIs (reboxetine in particular) have an effect on improving negative symptoms. However, we did not find evidence that NRIs have an effect on improving positive symptoms, cognitive functioning or incidence of nausea. One trial reported a benefit of reboxetine on quality of life scores.

\section{Conclusions}

The results of our review should be viewed with caution as the quality of evidence available is very low due to the small size of studies and poor quality of the trials. In order to make firm conclusions regarding the effectiveness of NRIs for people with schizophrenia we need larger and better quality trials of NRIs. These should be long term and look particularly at negative and cognitive symptoms as well as side-effects. 
SUMMARY OF FINDINGS

Summary of findings for the main comparison. Noradrenaline reuptake inhibitors versus placebo

Selective noradrenaline reuptake inhibitor (NRI) versus placebo in schizophrenia

Patient or population: adults with schizophrenia

Setting: inpatient and outpatient

Intervention: NRI

Comparison: placebo

\begin{tabular}{|c|c|c|c|c|c|c|}
\hline \multirow[t]{2}{*}{ Outcomes } & \multicolumn{2}{|c|}{ Anticipated absolute effects ${ }^{\star}(95 \% \mathrm{Cl})$} & \multirow{2}{*}{$\begin{array}{l}\text { Relative effect } \\
(95 \% \mathrm{CI})\end{array}$} & \multirow{2}{*}{$\begin{array}{l}\text { № of partici- } \\
\text { pants } \\
\text { (studies) }\end{array}$} & \multirow{2}{*}{$\begin{array}{l}\text { Quality of the } \\
\text { evidence } \\
\text { (GRADE) }\end{array}$} & \multirow[t]{2}{*}{ Comments } \\
\hline & $\begin{array}{l}\text { Risk with } \\
\text { placebo }\end{array}$ & Risk with NRI & & & & \\
\hline \multirow{2}{*}{$\begin{array}{l}\text { Mental state: significant response } \\
\text { or improvement in negative symp- } \\
\text { toms (PANSS negative subscale, high = } \\
\text { worse) - short term ( } 12 \text { weeks) }\end{array}$} & \multicolumn{2}{|c|}{ Study population } & \multirow{2}{*}{$\begin{array}{l}\text { RR } 3.17 \\
\text { (1.52 to } 6.58)\end{array}$} & \multirow{2}{*}{$\begin{array}{l}50 \\
(1 \mathrm{RCT})\end{array}$} & \multirow{2}{*}{$\begin{array}{l}\oplus \ominus \ominus \ominus \\
\text { VERY LOW } 123 \\
45\end{array}$} & \multirow{2}{*}{$\begin{array}{l}\text { Additional information } \\
\text { from average negative } \\
\text { symptoms score was } \\
\text { equivocal }\end{array}$} \\
\hline & 240 per 1000 & $\begin{array}{l}761 \text { per } 1000 \\
\text { (365 to } 1000)\end{array}$ & & & & \\
\hline \multirow[t]{2}{*}{$\begin{array}{l}\text { Mental state: specific - average posi- } \\
\text { tive symptoms score (PANSS positive } \\
\text { subscale, high = worse) - short term ( } 2 \\
\text { to } 12 \text { weeks) }\end{array}$} & - & $\begin{array}{l}\text { The mean PANSS pos- } \\
\text { itive score in the inter- } \\
\text { vention group was } 0.16 \\
\text { less ( } 0.96 \text { less to } 0.63 \\
\text { more) }\end{array}$ & \multirow[t]{2}{*}{-} & \multirow[t]{2}{*}{$\begin{array}{l}294 \\
\text { (5 RCTs) }\end{array}$} & \multirow[t]{2}{*}{$\begin{array}{l}\oplus \oplus \odot \odot \\
\text { LOW } 1,6\end{array}$} & $\begin{array}{l}\text { No data reported for our } \\
\text { prespecified outcome: } \\
\text { significant response or } \\
\text { improvement in positive } \\
\text { symptoms }\end{array}$ \\
\hline & & & & & & $\begin{array}{l}\text { SAPS and BPRS posi- } \\
\text { tive subscale outcomes } \\
\text { showed similar results }\end{array}$ \\
\hline \multirow{2}{*}{$\begin{array}{l}\text { Clinical global response: significant } \\
\text { response or improvement in glob- } \\
\text { al status (CGI-S, high = worse) - short } \\
\text { term ( } 4 \text { weeks) }\end{array}$} & \multicolumn{2}{|c|}{ Study population } & \multirow{2}{*}{$\begin{array}{l}\text { RR } 0.99 \\
\text { (0.45 to } 2.20 \text { ) }\end{array}$} & \multirow{2}{*}{$\begin{array}{l}28 \\
(1 \mathrm{RCT})\end{array}$} & \multirow{2}{*}{$\begin{array}{l}\oplus \ominus \ominus \ominus \\
\text { VERY LOW } 345 \\
79\end{array}$} & \multirow{2}{*}{$\begin{array}{l}\text { Additional informa- } \\
\text { tion from average clin- } \\
\text { ical global status score } \\
\text { found a similar lack of } \\
\text { effect }\end{array}$} \\
\hline & 467 per 1000 & $\begin{array}{l}462 \text { per } 1000 \\
(210 \text { to } 1000)\end{array}$ & & & & \\
\hline
\end{tabular}

Cognitive functioning: significant re- no data reported for this outcome sponse or improvement in cognitive functioning

Cognitive functioning: average com-

postive endpoint score (SMD, low

= favours NRI) - short term (2 to 12

- weeks)

\begin{tabular}{|c|c|}
\hline $\begin{array}{l}\text { The mean composite } \\
\text { cognitive functioning } \\
\text { SMD in the intervention }\end{array}$ & $\begin{array}{l}180 \\
\text { (4 RCTs) }\end{array}$ \\
\hline
\end{tabular}

SMD in the intervention

group was 0.04 standard 


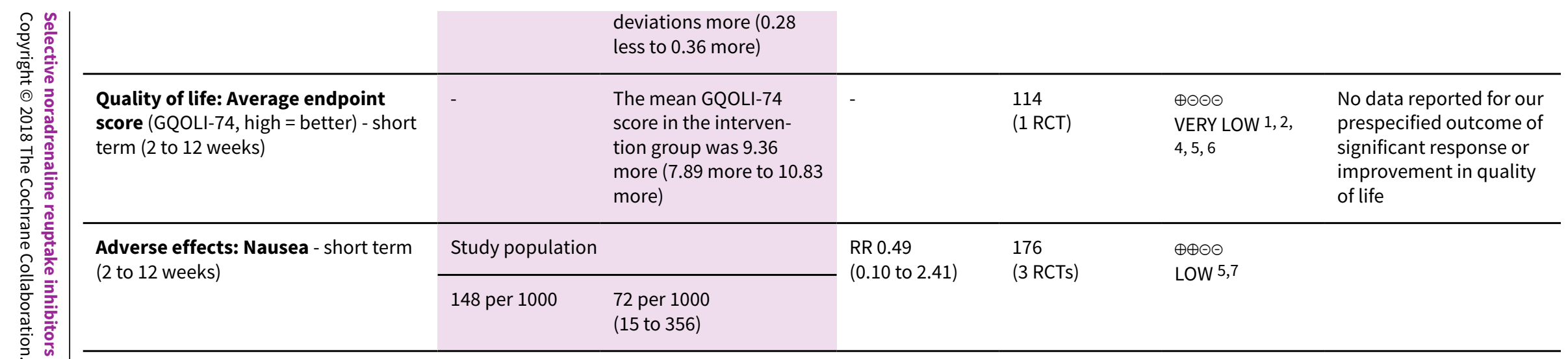

${ }^{*}$ The risk in the intervention group (and its $95 \%$ confidence interval) is based on the assumed risk in the comparison group and the relative effect of the intervention (and its $95 \% \mathrm{Cl})$.

Cl: Confidence interval; RR: Risk ratio; OR: Odds ratio; SMD: Standardised mean difference

\section{GRADE Working Group grades of evidence}

High quality: We are very confident that the true effect lies close to that of the estimate of the effect

Moderate quality: We are moderately confident in the effect estimate: The true effect is likely to be close to the estimate of the effect, but there is a possibility that it is substantially different

Low quality: Our confidence in the effect estimate is limited: The true effect may be substantially different from the estimate of the effect

Very low quality: We have very little confidence in the effect estimate: The true effect is likely to be substantially different from the estimate of effect

1 Imprecision: 'Serious' - Small sample size - Downgraded by 1 level

2 Risk of bias: 'Serious' - Methods of random sequence generation and allocation concealment are poorly described - Downgraded by 1 level

3 Risk of bias: 'Serious' - Evidence of selective reporting bias - Downgraded by 1 level

4 Inconsistency: 'No' - Only 1 study - Not downgraded

5 Publication bias: 'Strongly suspected' - Evidence of significant relevant unpublished or unusable results - Downgraded by 1 level

6 Indirectness: 'Serious' - Outcome of interest was not reported so a related outcome was substituted as a surrogate - Downgraded by 1 level

7 Imprecision: 'Serious' - Small sample size, confidence intervals include clinically significant benefits or harms - Downgraded by 1 level

8 Risk of bias: 'Serious' - Includes studies with high risk of attrition, reporting, and other sources of bias - Downgraded by 1 level

9 Indirectness: 'Serious' - Participants recruited specifically with depressive symptoms so unrepresentative of most patients with schizophrenia - Downgraded by 1 level 


\section{B A C K G R O U N D}

\section{Description of the condition}

Schizophrenia is frequently a chronic and disabling illness (Prudo 1987) but it has a variable course and patients present with a heterogeneous range of symptoms. These have been grouped into 'positive' and 'negative' categories (Crow 1980). Positive symptoms include psychotic phenomena such as hallucinations and delusions, and disorganised features including formal thought disorder, bizarre behaviour and inappropriate affect. Negative symptoms include affective flattening, lack of speech (alogia), lack of motivation (avolition) and inability to experience pleasure (anhedonia). In addition to the diagnostic symptoms associated with schizophrenia, it is now clear that it is also accompanied by cognitive deficits, particularly in working memory and attention (Bilder 2000; Weickert 2000). Cognitive and negative symptoms are difficult to treat with conventional antipsychotics and significantly impact on quality of life and social outcomes (Goldberg 2007; Green 1996; Makinen 2008).

\section{Description of the intervention}

The selective noradrenaline reuptake inhibitors (NRIs) were originally designed to specifically inhibit noradrenaline reuptake as part of the trend towards more selective targeting of antidepressant action that started with the development of the selective serotonin reuptake inhibitors (SSRIs) (Lopez-Munoz 2009). The pharmacology of NRIs differs from the SSRIs (e.g. fluoxetine) and serotonin-noradrenaline reuptake inhibitors (e.g. venlafaxine) by having minimal serotonergic effect.

The two main NRIs are atomoxetine and reboxetine. Atomoxetine (as tomoxetine) was originally trialled in the $1980 \mathrm{~s}$ as an antidepressant but development was discontinued by Eli Lilly to focus on its use in attention deficit hyperactivity disorder (ADHD) for which it was licensed in the mid-1990s (Preti 2002). Evidence for an antidepressant effect is largely negative (Fleurence 2009). Conversely, reboxetine was successfully launched by Pfizer as an antidepressant in the mid-1990s with claims for similar efficacy to SSRIs. However, recently it has been suggested that it is in fact less effective than other antidepressants (Cipriani 2009); and, when unpublished trials are taken into account, may in fact be no better (or even harmful) compared with placebo (Eyding 2010).

Reboxetine is used in two divided doses from $8 \mathrm{mg}$ to $12 \mathrm{mg}$ daily (BNF 2016) with a half-life of around 13 hours and it is primarily metabolised by the CYP3A4 isozyme of cytochrome P450 (Pfizer 2015). Atomoxetine is used in doses from $40 \mathrm{mg}$ to $120 \mathrm{mg}$ daily (BNF 2016). It is metabolised by the CYP2D6 isozyme of cytochrome $\mathrm{P} 450$ and has a half-life that ranges from 3.6 hours in extensive metabolisers to 21 hours in poor metabolisers (Eli Lilly 2015). The main side-effects of reboxetine and atomoxetine are loss of appetite, nausea, agitation, insomnia, dizziness, constipation, fatigue, dry mouth, sedation, sweating, and palpitations. Reboxetine is associated with postural hypotension and atomoxetine with hypertension. Rare but more serious complications include liver damage with atomoxetine and suicidal thoughts with both (BNF 2016).

\section{How the intervention might work}

In contrast with other stimulant medication (such as amphetamines), NRIs increase prefrontal dopamine without significantly affecting subcortical dopamine levels (Bymaster 2002; Masana 2011; Marcus 2010), which leads to a substantially decreased abuse potential (Jasinski 2008). This makes NRIs an attractive candidate for treating schizophrenia, and particularly negative and cognitive symptoms (Apud 2007b; Friedman 1999; Raedler 2004), since it is thought that subcortical dopamine excess underlies positive symptoms (hence the efficacy of dopamine receptor blockade with antipsychotic medication; Seeman 1975) while relative prefrontal dopamine deficit is hypothesised to contribute to negative symptoms and cognitive dysfunction (Knable 1997).

Meta-analyses have concluded that antidepressants used as add-on therapy show a moderate beneficial effect on negative symptoms in schizophrenia (Rummel-Kluge 2006; Singh 2010). Amphetamines have potent dopaminergic and noradrenergic activity and there is some evidence for a beneficial effect on negative and cognitive symptoms (Daniel 1991; Nolte 2004). In ADHD (Chamberlain 2007; Faraone 2005) and in animal models (Seu 2009), atomoxetine has been associated with improved cognitive function.

\section{Why it is important to do this review}

Current recommendations for pharmacological management of negative symptoms in schizophrenia are limited and there are no strategies recommended for the pharmacological treatment of cognitive symptoms. While there is some evidence of a small benefit of certain atypical antipsychotics over typical antipsychotics the effect is small (e.g. an effect size of 0.3 for amisulpride) (Leucht 2009). Augmentation of clozapine can also give some additional benefit (e.g. an effect size of 0.4 for lamotrigine) (Tiihonen 2009). However, SSRIs or mirtazapine are usually recommended first-line for negative symptoms (Barnes 2011; Maudsley 2015). The overall benefit of antidepressants on negative symptoms is still quite small (an effect size of 0.5 overall, 0.4 for fluoxetine specifically) (Singh 2010) with some evidence of a larger benefit with mirtazapine and mianserin (an effect size of 0.8 to 0.9) (Hecht 2012; Kishi 2014). The relatively larger benefit of mirtazapine and mianserin is interesting as these drugs act as alpha-2 autoreceptor and heteroreceptor antagonists promoting efflux of not only serotonin but also noradrenaline.

There have been a number of trials of NRIs, both atomoxetine and reboxetine, as add-on therapy for schizophrenia, all largely negative in their findings but each underpowered with small sample sizes. We sought to combine the data from these trials to increase the power and assess whether the totality of evidence favours the use of NRIs in schizophrenia, given strong theoretical reasons to suspect that they would be beneficial for negative and cognitive symptoms in particular.

\section{O B J E C T IVES}

To investigate the effects of selective noradrenaline reuptake inhibitors (NRIs), compared with a placebo or control treatment, for people with schizophrenia. 


\section{METHODS}

\section{Criteria for considering studies for this review \\ Types of studies}

All relevant randomised controlled trials. If a trial was described as 'double blind' but implied randomisation, we would have included it in a sensitivity analysis. We excluded quasi-randomised studies, such as those allocating by alternate days of the week. Where people were given additional treatments within a selective noradrenaline reuptake inhibitor (NRI) trial, we only included data if the adjunct treatment was evenly distributed between groups and it was only the NRI treatment that was randomised.

\section{Types of participants}

Trials with a majority of participants ( $\geq 70 \%$ ) with schizophrenia or related disorders (including schizophreniform disorder, schizoaffective disorder, and delusional disorder, but excluding bipolar disorder or psychotic depression) by any means of diagnosis.

\section{Types of interventions}

\section{Selective noradrenaline reuptake inhibitor treatment}

Defined as treatment with atomoxetine, reboxetine or other NRI by any dose or mode of administration but for a minimum duration of one week; most likely as an augmentation strategy but not excluding studies as monotherapy; excluding non-selective compounds such as those also significantly affecting dopamine or serotonin reuptake.

\section{Placebo treatment}

Defined as treatment with an inactive compound.

\section{Active control treatment}

Defined as treatment with an active compound other than an NRI.

\section{Types of outcome measures}

We grouped the outcomes into brief (less than two weeks), shortterm (two to 12 weeks), medium-term (13 to 26 weeks), and longterm (over 26 weeks) durations. If outcomes were available for multiple time-points within the same overall duration we used the longest duration data (e.g. if there were outcomes for 2 weeks, 4 weeks, and 8 weeks, and 24 weeks then we used the data from 8 weeks when considering the short term and 24 weeks for the medium term).

\section{Primary outcomes}

\section{Mental state}

Specific - significant response or improvement in negative symptoms as defined by each study.

\section{Cognitive functioning}

Significant response or improvement in clinical scale of cognitive functioning as defined by each study.

\section{Quality of life}

Significant response or improvement in quality of life as defined by each study.

\section{Clinical global response}

Significant response or improvement in clinical global status as defined by each study.

\section{Secondary outcomes}

\section{Mental state}

1.1 General: significant response or improvement in general/overall symptoms as defined by each study

1.2 General: average general/overall symptoms score

1.3 Specific: significant response or improvement in positive symptoms as defined by each study

1.4 Specific: average positive symptoms score

1.5 Specific: average negative symptoms score

1.6 Specific: significant response or improvement in mood as defined by each study

1.7 Specific: average mood score

1.8 Specific: average score for other symptoms

\section{Cognitive functioning}

2.1 Average clinical scale of cognitive functioning score

2.2 Significant response or improvement in composite cognitive functioning

2.3 Average composite cognitive functioning score

2.4 Significant response or improvement in working memory as defined by each study

2.5 Average working memory score

2.6 Significant response or improvement in reasoning/problem solving as defined by each study

2.7 Average reasoning/problem solving score

2.8 Significant response or improvement in speed of processing as defined by each study

2.9 Average speed of processing score

2.10 Significant response or improvement in attention as defined by each study

2.11 Average attention score

2.12 Significant response or improvement in verbal learning/ memory as defined by each study

2.13 Average verbal learning/memory score

2.14 Significant response or improvement in visual learning/ memory as defined by each study

2.15 Average visual learning/memory score

\section{Quality of life}

3.1 Average quality of life score

\section{Clinical global response}

4.1 Average clinical global status score

4.2 Relapse as defined by each study

\section{Service utilisation outcomes}

5.1 Days in hospital

5.2 Admission to hospital

\section{Leaving the study early}

6.1 for any reason

6.2 due to specific event (e.g. adverse event)

\section{Adverse effects}

7.1 Death 
7.2 General adverse events and side-effects

7.3 Significant extrapyramidal side-effects/movement disorder as defined by each study

7.4 Average extrapyramidal side-effects/movement disorder score

7.5 Incidence of use of antiparkinson medication

7.6 Cumulative dosage of antiparkinson medication

7.7 Average weight gain

7.8 Significant weight gain as defined by each study

\section{Satisfaction with treatment}

8.1 Significant improvement in satisfaction with treatment as defined by each study

8.2 Average satisfaction with treatment score

8.3 General impression of carer/other

\section{Social or general functioning}

9.1 Significant response or improvement in social functioning as defined by each study

9.2 Average social functioning score

9.3 Occupational status

\section{Economic outcomes}

\section{1. 'Summary of findings' table}

We have used the GRADE approach to interpret findings (Schünemann 2008) and used GRADE profiler (GRADE PRO) to import data from Review Manager 5 (Review Manager) to create 'Summary of findings' tables. These tables provide outcomespecific information concerning the overall quality of evidence from each included study in the comparison, the magnitude of effect of the interventions examined, and the sum of available data on all outcomes we rate as important to patient-care and decision making. We intended to select the following main outcomes for inclusion in the 'Summary of findings' table.

1. Mental state: Specific - significant response or improvement in negative symptoms as defined by each study

2. Mental state: Specific - significant response or improvement in positive symptoms as defined by each study

3. Clinical global response significant response or improvement in clinical global status as defined by each study

4. Cognitive functioning - significant response or improvement in cognitive functioning as defined by each study

5. Cognitive functioning - average composite cognitive functioning score

6. Quality of life - significant response or improvement in quality of life as defined by each study

7. Specific adverse effect - incidence of nausea as defined by each study

\section{Search methods for identification of studies}

\section{Electronic searches}

\section{Cochrane Schizophrenia Group's Trials Register}

The Trials Search Coordinator (TSC) searched the Cochrane Schizophrenia Group's Registry of Trials (20 August 2014; 17 November 2015; 7 February 2017) using the following search strategy:

(*atomoxetine* or *attentin* or *beloxepin* or *davedax* or ${ }^{*}$ edonax* or *edronax* or *esreboxetine ${ }^{\star}$ or *FCE $20124^{*}$ or * FCE $21684^{*}$ or * $\mathrm{LY} 139602^{*}$ or * $\mathrm{LY} 139603^{*}$ or *nisoxetine* or * norebox ${ }^{*}$ or * Org $4428^{*}$ or * prolift* or * ${ }^{\star}$ reboxetine* or * ${ }^{*}$ olvex* or *SPN

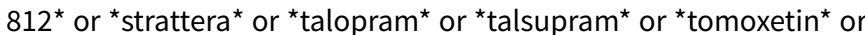
${ }^{\star}$ vestra* or ${ }^{\star} \mathrm{NRI}^{\star}$ or * noradrenaline reuptake ${ }^{\star}$ or * norepinephrine reuptake*) in Title, Abstract and Keyword Fields of REFERENCE and Intervention Field of Study

In such study-based registers, searching the major concept retrieves all the synonyms and relevant studies because all the studies have already been organised based on their interventions and linked to the relevant topics (Shokraneh 2017).

This register is compiled by systematic searches of major resources (AMED, BIOSIS, CINAHL, ClinicalTrials.Gov, Embase, MEDLINE, PsycINFO, PubMed, WHO ICTRP) and their monthly updates; ProQuest Dissertations and Theses A\&I and its quarterly update; Chinese databases (CBM, CNKI, and Wanfang) and their annual updates; handsearches; grey literature; and conference proceedings (see Group's Module). There are no language, date, document type, or publication status limitations for inclusion of records in the register.

For previous searches, see Appendix 1.

\section{Searching other resources}

\section{Reference searching}

We inspected references of all identified studies for further relevant studies.

\section{Personal contact}

We attempted to contact the corresponding author of each included study for information regarding unpublished trials.

\section{Data collection and analysis}

\section{Selection of studies}

Two authors (PM and $\mathrm{JH}$ ) independently inspected citations from the searches and identified relevant abstracts. We obtained full reports of the abstracts meeting the review criteria or references/ abstracts that authors disagreed on; and both review authors inspected them. We would have contacted the authors of studies for clarification if it had not been possible to resolve disagreement by discussion.

\section{Data extraction and management}

\section{Extraction}

Two review authors (PM and MP) independently extracted data from all included studies, discussed any disagreement and documented decisions. We extracted data presented only in graphs and figures whenever possible, but included only if both review authors independently had the same result. We made attempts to contact authors through an open-ended request in order to obtain missing information or for clarification whenever necessary. If studies were multicentre we attempted to extract data relevant to each component centre separately.

\section{Management}

\subsection{Forms}

We extracted data onto standard, simple forms. Information included relevant study details including trial intervention (e.g. atomoxetine), participant numbers and diagnoses (including 
clinical subgroups and illness duration), baseline symptom severity (and differences between arms), demographics (age, sex), medication (concomitant antipsychotic, NRI dosage), trial duration, outcome measures, and trial quality. Baseline severity was estimated based on Clinical Global Impression (CGI) descriptors (Guy 1976); and utilising correlations between other measures and the CGI when the latter was not reported (Leucht 2005b; Levine 2013; Rabinowitz 2006).

\subsection{Scale-derived data}

We included continuous data from rating scales only if:

a. the psychometric properties of the measuring instrument had been described in a peer-reviewed journal (Marshall 2000); and b. the measuring instrument had not been written or modified by one of the trialists for that particular trial.

Ideally, the measuring instrument should either be i. a self-report or ii. completed by an independent rater or relative (not the therapist). We realise that this is not often reported clearly, and noted in Description of studies if this was the case or not.

\subsection{Endpoint versus change data}

There are advantages of both endpoint and change data. Change data can remove a component of between-person variability from the analysis. On the other hand, calculation of change needs two assessments (baseline and endpoint) which can be difficult in unstable and difficult-to-measure conditions such as schizophrenia. We decided to preferentially use endpoint data if both endpoint and change data were available, if the standard deviation (SD) of end scores needed to be imputed but the SD for change scores was available then we used the latter in order to minimise the use of imputation. We combined endpoint and change data from different trials in analyses using mean differences (MD) where possible.

\subsection{Skewed data}

Continuous data on clinical and social outcomes are often not normally distributed. To avoid the pitfall of applying parametric tests to non-parametric data, we applied the following standards to relevant data before inclusion.

a) Standard deviations (SDs) and means were reported in the paper or obtainable from the authors (this is not possible if the SD is imputed).

b) When a scale starts from the finite number zero, the SD, when multiplied by two, is less than the mean (as otherwise the mean is unlikely to be an appropriate measure of the centre of the distribution (Altman 1996)).

c) If a scale started from a positive value (such as the Positive and Negative Syndrome Scale, PANSS, which can have values from 30 to 210), we modified the calculation described above to take the scale starting point into account. In these cases skew is present if $2 S D>(S$ - Smin), where S is the mean score and Smin is the minimum score. Endpoint scores on scales often have a finite start and end point and these rules can be applied. When continuous data are presented on a scale that includes a possibility of negative values (such as change data), it is difficult to tell whether data are skewed or not. Skewed data pose less of a problem when looking at means if the sample size is large and would have been entered into syntheses. We entered skewed endpoint data from studies of less than 200 participants as Additional tables rather than into analyses.
Although we could not determine whether change scores were skewed we attempted to calculate endpoint scores from these where possible and assessed these for evidence of skew. While using the associated change scores rather than endpoint scores could correct the skew it was not possible to test this so we performed a sensitivity analysis where we excluded those change scores which were associated with skewed endpoint scores.

\subsection{Common measure}

To facilitate comparison between trials, we converted variables that could be reported in different metrics, such as days in hospital (mean days per year, per week or per month) to a common metric (e.g. mean days per month).

\subsection{Conversion of continuous to binary}

We made efforts to convert outcome measures to dichotomous data where sufficient information was available. This can be done by identifying cut-off points on rating scales and dividing participants accordingly into 'clinically improved' or 'not clinically improved'. It is generally assumed that if there is a $50 \%$ reduction in a scale-derived score such as the Brief Psychiatric Rating Scale (BPRS, Overall 1962) or the PANSS (Kay 1986), this could be considered as a clinically significant response (Leucht 2005a; Leucht 2005b). If data based on these thresholds were not available, we used the primary cut-off presented by the original authors. We did not use methods to estimate dichotomous outcomes directly from summary statistics (mean and SD) of continuous data. Even if we had been able to convert continuous data to dichotomous data, we would also have analysed the continuous data separately as it provides additional, complementary information to dichotomous data.

\subsection{Direction of graphs}

Where possible, we entered data in such a way that the area to the left of the line of no effect indicates a favourable outcome for NRIs. Where keeping to this made it impossible to avoid outcome titles with clumsy double-negatives (e.g. 'Not un-improved') we reported data where the left of the line indicates an unfavourable outcome and noted this in the relevant graphs.

\subsection{Composite cognitive scores}

We used composite scores for cognitive functioning as defined in each study. If composite scores were not reported directly then we calculated these as the mean of the individual effect sizes of outcomes presented in that study where possible (after Wykes 2011).

\section{Assessment of risk of bias in included studies}

Again, two review authors (PM and MP) worked independently to assess risk of bias by using criteria described in the Cochrane Handbook for Systematic Reviews of Interventions to assess trial quality (Higgins 2011). This set of criteria is based on evidence of associations between overestimate of effect and high risk of bias of the article such as sequence generation, allocation concealment, blinding, incomplete outcome data and selective reporting.

If the raters disagreed, they made the final rating decision by consensus. Where inadequate details of randomisation and other characteristics of trials were provided, we contacted authors of the studies in order to obtain further information. We report nonconcurrence in quality assessment, but if disputes arose as to 
which category a trial was to be allocated, again we resolved by discussion.

The level of risk of bias is noted in both the text of the review and in the Summary of findings for the main comparison.

\section{Measures of treatment effect}

\section{Binary data}

For binary outcomes we calculated a standard estimation of the risk ratio (RR) and its $95 \%$ confidence interval $(\mathrm{Cl})$. It has been shown that RR is more intuitive than odds ratios (Boissel 1999); and that odds ratios tend to be interpreted as RR by clinicians (Deeks 2000). Further illustrative comparative risks are found in the Summary of findings for the main comparison.

\section{Continuous data}

For continuous outcomes we estimated mean difference (MD) between groups where possible. However, we were particularly interested in measures of cognitive function and traditionally a number of different tests have been used to measure each cognitive domain. Frequently these are only really interpretable, or meaningful, when normalised. Therefore, we used effect size measures (standardised mean difference, SMD) to combine all tests reported by a study that measure the same cognitive domain and also to calculate an overall composite cognitive score for that study.

We pooled the SMD effect size measures using generic inverse variance. The mean effect size approach does not take into account covariation and therefore leads to conservative estimates (Wykes 2011) and we included effect sizes calculated this way in a sensitivity analysis. We have interpreted these estimates of composite effect size cautiously and would have contrasted them with outcomes derived from individual cognitive tests if available. When effect sizes are relatively homogeneous (as would reasonably be assumed for tests measuring the same cognitive domain), the simple mean is a suitable estimate even without taking into account the covariance structure (Marin-Martinez 1999).

We have drawn our cognitive domains of interest in schizophrenia from the MATRICS-NIMH review and assigned cognitive tests to domains as outlined in that paper (Nuechterlein 2004). For cognitive function scores which are not covered in Nuechterlein 2004 we referred to the primary literature to assign the best fitting cognitive domain (if any). We understand that there are significant assumptions involved in combining different outcomes scales as SMD (Higgins 2011); and we have interpreted this cautiously and only used the SMD where there is not a substantial level of heterogeneity (see Subgroup analysis and investigation of heterogeneity).

\section{Unit of analysis issues}

\section{Cluster trials}

Studies increasingly employ 'cluster randomisation' (such as randomisation by clinician or practice) but analysis and pooling of clustered data poses problems. Firstly, authors often fail to account for intra-class correlation in clustered studies, leading to a 'unit of analysis' error whereby P values are spuriously low, confidence intervals unduly narrow and statistical significance overestimated (Divine 1992). This causes type I errors (Bland 1997; Gulliford 1999).
If clustering had not been accounted for in primary studies, we would have presented data in a table, with a $\left({ }^{*}\right)$ symbol to indicate the presence of a probable unit of analysis error. We would have contacted first authors of studies to obtain intra-class correlation coefficients (ICCS) for their clustered data and adjusted for this by using accepted methods (Gulliford 1999). If clustering had been incorporated into the analysis of primary studies, we would have presented these data as if from a non-cluster randomised study, but adjusted for the clustering effect.

We have sought statistical advice and have been advised that the binary data as presented in a report should be divided by a 'design effect'. This is calculated using the mean number of participants per cluster $(\mathrm{m})$ and the ICC [Design effect $=1+(\mathrm{m}-1)^{\star} \mathrm{ICC}$ ] (Donner 2002). If the ICC is not reported, it will be assumed to be 0.1 (Ukoumunne 1999).

If cluster studies had been appropriately analysed taking into account ICCs and relevant data had been documented in the report, we would have been able to synthesise with other studies using the generic inverse variance technique.

\section{Cross-over trials}

A major concern of cross-over trials is the carry-over effect. It occurs if an effect (pharmacological, physiological or psychological) of the treatment in the first phase is carried over to the second phase. As a consequence, on entry to the second phase the participants can differ systematically from their initial state despite a wash-out phase. For the same reason cross-over trials are not appropriate if the condition of interest is unstable (Elbourne 2002). As both effects are very likely in severe mental illness, we would have only used data from the first phase of cross-over studies.

\section{Studies with multiple treatment groups}

Where a study involved more than two treatment arms, if relevant we presented the additional treatment arms in comparisons. For binary data we simply added and combined within the two-bytwo table. If data were continuous we would have combined data following the formula in section 7.7.3.8 (Combining groups) of the Cochrane Handbook for Systematic Reviews of Interventions. We would not reproduce data that is not relevant to this review.

\section{Dealing with missing data}

\section{Overall loss of credibility}

At some degree of loss of follow-up, data must lose credibility (Xia 2009). We chose that, for any particular outcome, should more than $50 \%$ of data be unaccounted for, we would not reproduce these data or use them within analyses, except for the outcome of leaving the study early. If, however, more than $50 \%$ of those in one arm of a study were lost, but the total loss was less than $50 \%$, we would mark such data with $\left(^{*}\right)$ to indicate that such a result may well be prone to bias.

\section{Binary}

In the case where attrition for a binary outcome is between $0 \%$ and $50 \%$ and where these data are not clearly described, data are presented on a 'once-randomised-always-analyse' basis (an intention-to-treat analysis). Those leaving the study early are assumed to have the 'worst case scenario' (e.g. no clinical response). For the outcomes of death and adverse effects we used 
the rate of those who stayed in the study - in that particular arm of the trial - for those who did not and undertook a sensitivity analysis comparing 'completer' data only with the intention-totreat analysis. Where the denominator for withdrawals was not clear we included all those in that arm of the study who did not withdraw for other reasons.

\section{Continuous}

\subsection{Attrition}

In the case where attrition for a continuous outcome was between $0 \%$ and $50 \%$ and completer-only data were reported, we reproduced these.

\subsection{Standard deviations}

If SDs were not reported, we first tried to obtain the missing values from the authors. If not available, where there were missing measures of variance for continuous data, but an exact standard error (SE) and Cls were available for group means, and either $\mathrm{P}$ value or $t$ value available for differences in mean, we could calculate them according to the rules described in theCochrane Handbook for Systematic Reviews of Interventions (Higgins 2011). When only the $\mathrm{SE}$ is reported, SDs are calculated by the formula SD $=\mathrm{SE} * \sqrt{ }(\mathrm{n})$. Chapters 7.7.3 and 16.1.3 of the Cochrane Handbook for Systematic Reviews of Interventions present detailed formulae for estimating SDs from $\mathrm{P}$ values, $\mathrm{t}$ or $\mathrm{F}$ values, $\mathrm{Cls}$, ranges or other statistics (Higgins 2011). If these formulae did not apply, we calculated the SDs according to an imputation method which is based on the SDs of the other included studies (Furukawa 2006). An exception to this was when we were imputing the SD for change scores when we already had the SD for endpoint data from that study: in this case we assumed a correlation between baseline and endpoint scores of 0.5 used the formulae in the Cochrane Handbook for Systematic Reviews of Interventions (after Singh 2010). We intended to validate the use of a correlation of 0.5 by deriving estimates from other studies where possible and including these empirical estimates of correlation in a sensitivity analysis. For imputing the SD for endpoint data where we have baseline and change scores we used the baseline SD and examined this assumption in a sensitivity analysis.

Although these imputation strategies can introduce error, the alternative would be to exclude a given study's outcome and thus to lose information. We nevertheless examined the validity of the imputations in a sensitivity analysis excluding imputed values.

\subsection{Last observation carried forward}

We anticipated that in some studies the method of last observation carried forward (LOCF) would be employed within the study report. As with all methods of imputation to deal with missing data, LOCF introduces uncertainty about the reliability of the results (Leucht 2007). Therefore, where LOCF data were used in the trial, if less than $50 \%$ of the data had been assumed we reproduced these data if completer-only data were not available, and indicated that they were the product of LOCF assumptions.

\section{Assessment of heterogeneity}

\section{Clinical heterogeneity}

We considered all included studies initially, without seeing comparison data, to judge clinical heterogeneity. We then simply inspected all studies for clearly outlying populations or situations which we had not predicted would arise. When such situations or participant groups arose, we fully discussed these.

\section{Methodological heterogeneity}

We considered all included studies initially, without seeing comparison data, to judge methodological heterogeneity. We simply inspected all studies for clearly outlying methods which we had not predicted would arise. When such methodological outliers arose, we fully discussed these.

\section{Statistical heterogeneity}

\subsection{Visual inspection}

We visually inspected graphs to investigate the possibility of statistical heterogeneity.

\subsection{Employing the $I^{2}$ statistic}

We investigated heterogeneity between studies by considering the $\mathrm{I}^{2}$ statistic alongside the $\mathrm{Chi}^{2} \mathrm{P}$ value. The $\mathrm{I}^{2}$ statistic provides an estimate of the percentage of inconsistency thought to be due to chance (Higgins 2003). The importance of the observed value of $\mathrm{I}^{2}$ depends on i. magnitude and direction of effects and ii. strength of evidence for heterogeneity (e.g. $\mathrm{P}$ value from $\mathrm{Chi}^{2}$ test, or a confidence interval for $I^{2}$ ). We interpreted an $I^{2}$ estimate greater than or equal to $50 \%$ accompanied by a statistically significant $\mathrm{Chi}^{2}$ statistic as evidence of substantial levels of heterogeneity (Section 9.5.2 - Higgins 2011). When substantial levels of heterogeneity were found in the outcome, we explored some potential reasons for heterogeneity (Subgroup analysis and investigation of heterogeneity).

\section{Assessment of reporting biases}

Reporting biases arise when the dissemination of research findings is influenced by the nature and direction of results (Egger 1997). These are described in Section 10 of the Cochrane Handbook for Systematic Reviews of Interventions (Higgins 2011). We are aware that funnel plots may be useful in investigating reporting biases but are of limited power to detect small-study effects (Sterne 2011). We did not use funnel plots for outcomes where there were 10 or fewer studies, or where all studies were of similar size. In other cases, if funnel plots were possible we would have sought statistical advice in their interpretation.

Where study protocols have been published, we compared these to the reported results to look for outcome reporting bias. Otherwise we looked for unreported outcome measures mentioned in the 'Methods' section of the study.

\section{Data synthesis}

We understand that there is no closed argument for preference for use of fixed-effect or random-effects models. The random-effects method incorporates an assumption that the different studies are estimating different, yet related, intervention effects. This often seems to be true to us and the random-effects model takes into account differences between studies even if there is no statistically significant heterogeneity. There is, however, a disadvantage to the random-effects model: it puts added weight onto small studies which often are the most biased ones. Depending on the direction of effect, these studies can either inflate or deflate the effect size. We chose random-effects models for all analyses but we examined the consequences of our choice in sensitivity analyses. 


\section{Subgroup analysis and investigation of heterogeneity}

We only conducted formal subgroup or sensitivity analyses where there were more than three trials available but we investigated all outcomes for sources of heterogeneity.

\section{Subgroup analyses}

1.1 Individual noradrenaline reuptake inhibitors (NRIs)

As well as reporting findings from NRIs combined as a class, we also analysed data from the two main NRIs (reboxetine and atomoxetine) separately.

\subsection{Clinical state, stage or problem}

We are interested in making sure that information is as relevant to the current care of people with schizophrenia as possible so intended to clearly highlight the current clinical state (acute, early post-acute, partial remission, remission) as well as the stage (prodromal, first episode, early illness, persistent) and as to whether the studies primarily focused on people with particular problems (for example, negative symptoms, treatment-resistant illnesses).

\subsection{Participant age}

We grouped each study by the age range of the majority of participants included (under 18 years, 18 to 60 years, over 60 years) and analysed separately. If the proportion within each age range was not available then we allocated studies by mean age.

\section{Investigation of heterogeneity}

We reported if inconsistency was high. First, we investigated whether data were entered correctly. Second, if data were correct, we visually inspected the graph and removed outlying studies to see if homogeneity was restored. If substantial heterogeneity remained, we discussed this but did not perform further investigation of potential causes of heterogeneity (e.g. meta-regression) beyond our planned subgroup and sensitivity analyses.

When unanticipated clinical or methodological heterogeneity was obvious, we simply stated hypotheses regarding these for future reviews or versions of this review. We did not undertake analyses relating to this.

\section{Sensitivity analysis}

\section{Implication of randomisation}

We aimed to include trials in a sensitivity analysis if they were described in some way as to imply randomisation. We included these studies and if there was no substantive difference when the implied randomised studies were added to those with better description of randomisation, then we used all relevant data from these studies.

\section{Assumptions for lost binary data}

Where assumptions had to be made regarding people lost to followup (see Dealing with missing data), we compared our intentionto-treat assumptions with completer data only. If there was a substantial difference, we reported results and discussed them but continued to employ our assumption.

\section{Risk of bias}

We analysed the effects of excluding trials that were judged to be at high risk of bias across one or more of the domains of randomisation, allocation concealment, blinding and outcome reporting for the meta-analysis. If the exclusion of trials at high risk of bias did not substantially alter the direction of effect or the precision of the effect estimates, then we entered relevant data from these trials in the analysis.

\section{Imputed values}

We undertook a sensitivity analysis to assess the effects of including data from trials where we used imputed values (see Dealing with missing data). If we noted substantial differences in the direction or precision of effect estimates in these sensitivity analyses then we did not pool data from the imputed trials with the other trials contributing to the outcome, but presented them separately. Where imputed SDs depend on an assumption of 0.5 correlation of baseline to endpoint scores, we attempted to derive an estimate of this correlation coefficient empirically from included studies and to compare this estimate with the assumption of 0.5 in a sensitivity analysis.

\section{Mean effect sizes for composite cognitive scores}

Where we calculated mean effect sizes to create composite cognitive scores, we attempted to examine the effect of including or excluding these from other studies where composite scores were reported directly. Where there were substantial differences, we presented these data separately.

\section{Fixed-effect and random-effects}

We synthesised all data using a random-effects model but we also reported outcomes using a fixed-effect model to evaluate whether the greater weights assigned to larger trials with greater event rates altered the significance of the results compared with the more evenly distributed weights in the random-effects model.

\section{Skew}

Where we used change scores, but these were associated with skewed endpoint scores, we examined the effect of excluding these change scores.

\section{RES U L T S}

\section{Description of studies}

\section{Results of the search}

See Figure 1 for the search flow diagram. We identified 80 distinct records from searches up to 2012, a further six records repeating the search in 2014, a further three records in 2015, and a further 17 records in 2017. In addition we identified one further record when the search criteria were broadened in 2015 . We also identified three records through handsearching citations, responses from authors contacted, and other sources. 
Figure 1. Study flow diagram.

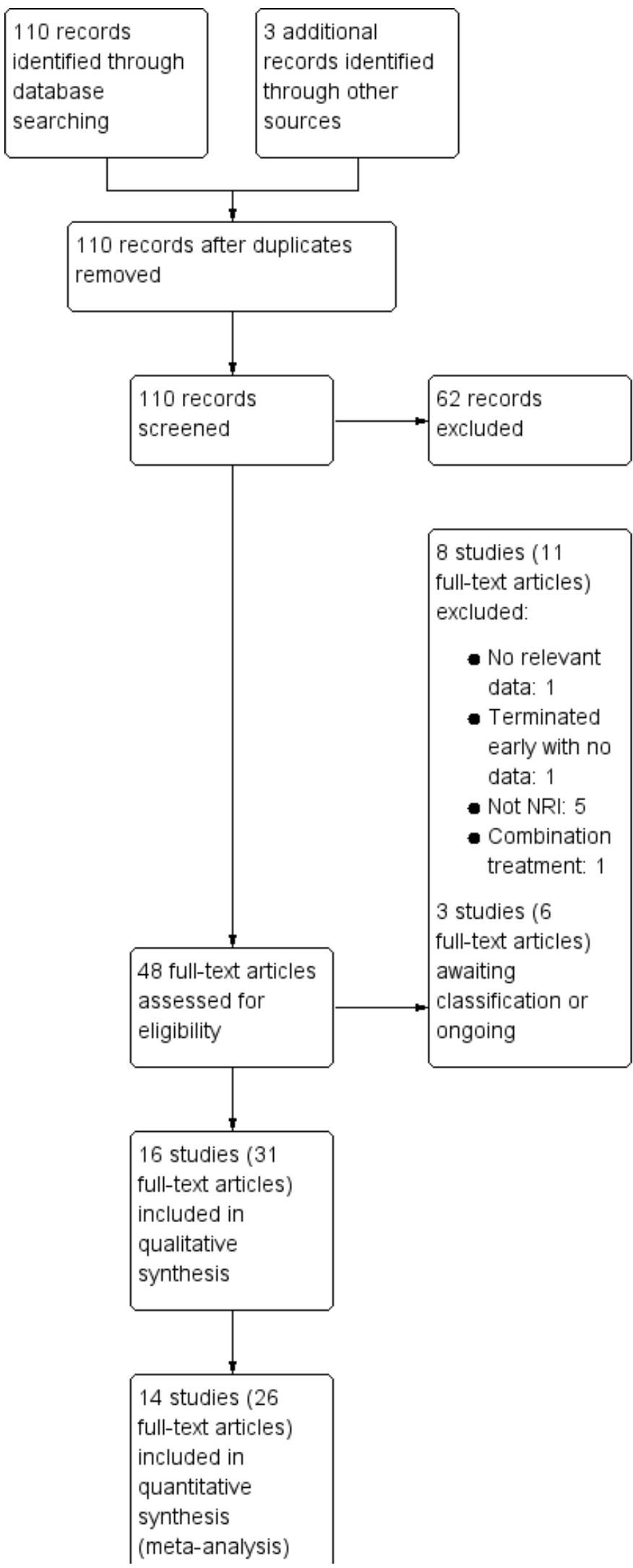


Figure 1. (Continued)

$$
\mid \begin{aligned}
& \text { syrutuesıs } \\
& \text { (meta-analysis) }
\end{aligned}
$$

Of the 110 distinct records identified 62 were excluded based on the title and abstract alone as they did not meet the inclusion criteria (i.e. they were not RCTs of NRIs). We obtained the full text of 48 records. Of these, 16 distinct studies were included in the review, representing 31 records. Eight distinct studies were excluded as they did not meet the inclusion criteria, representing 11 records. There are two studies awaiting classification (see also Characteristics of studies awaiting classification), which represent five records; and there is one ongoing study (see also Characteristics of ongoing studies). All records identified from sources other than the main search were for studies also identified by records in the main search.

In 2015 we broadened the search criteria to include further substances we identified as selective noradrenaline reuptake inhibitors, including viloxazine which we had previously included in the search under the name 'SPN-812'. We identified one study utilising viloxazine (Kurland 1981). Viloxazine is a selective noradrenaline reuptake inhibitor that was manufactured by $\mathrm{ICl}$ and used as an antidepressant in Europe until being withdrawn in the early 2000s. It has since been investigated by Supernus Pharmaceuticals as an antidepressant and treatment for ADHD as SPN-809 and SPN-812 respectively.

\section{Studies awaiting classification}

We have identified one RCT of atomoxetine which has been terminated (Shekhar 2005) and another RCT which has been completed (Tamminga 2009) but could find no further information on the results. See Characteristics of studies awaiting classification.

\section{Ongoing studies}

We have identified one RCT of reboxetine started in 2006 for which we have not been able to determine any further information (Baranchik 2006). Therefore it is unclear if this trial is ongoing or terminated. See Characteristics of ongoing studies.

\section{Included studies}

\subsection{Methods}

We included sixteen studies; details of individual studies are listed in the Characteristics of included studies table. All were randomised parallel trials and double-blind placebo controlled except for one randomised open trial (Yu 2012); while two trails also had a citalopram arm (Hinkelmann 2013; Usall 2014). All studies were published in English except for three trials in Chinese (Li 2008; Yu 2012; Zhao 2013).

Most trials were of short duration with eleven lasting between two and 12 weeks (Eli Lilly 2006; Friedman 2008; Hinkelmann 2013; Kelly 2009; Kurland 1981; Li 2008; Poyurovsky 2003; Poyurovsky 2007; Sacco 2009; Schutz 2001; Shafti 2015); and the remaining five were medium term, lasting 13 to 26 weeks (Ball 2011; Ganguli 2008; Usall 2014; Yu 2012; Zhao 2013).

\subsection{Setting}

Where reported, seven trials included only inpatients (Li 2008; Poyurovsky 2003; Poyurovsky 2007; Schutz 2001; Shafti 2015; Yu 2012; Zhao 2013); two only outpatients (Ball 2011; Friedman 2008); and one reported enrolling both (Kelly 2009).

\subsection{Participants}

Most studies included only patients diagnosed with schizophrenia (Eli Lilly 2006; Friedman 2008; Hinkelmann 2013; Kurland 1981; Schutz 2001; Shafti 2015; Usall 2014; Yu 2012; Zhao 2013) with some also including schizoaffective disorder (Ball 2011; Ganguli 2008; Kelly 2009; Sacco 2009) or schizophreniform disorder (Poyurovsky 2003; Poyurovsky 2007). Where reported, all participants were adults aged 18 to 65 years with no evidence of participants aged under 18 years or over 65 years being enrolled in trials. All studies used the Diagnostic and Statistical Manual of Mental Disorders, Fourth Edition (DSM-IV) except two using the Chinese Classification of Mental Disorders, Third Edition (CCMD-3) (Li 2008; Yu 2012), one using the International Classification of Diseases, 10th Revision (ICD-10) (Zhao 2013), and one not reporting the criteria used (Kurland 1981).

Duration of illness ranged from 10 months (Yu 2012), one to six years (Li 2008; Poyurovsky 2003; Poyurovsky 2007; Zhao 2013), to over 10 years (Hinkelmann 2013; Kelly 2009; Usall 2014). Although not reporting overall duration of illness two studies indicated at least six months or two years of illness (Ball 2011 and Shafti 2015 respectively). Three studies reported including stable patients (Ball 2011; Sacco 2009; Zhao 2013); and three reported including chronic patients (Li 2008; Schutz 2001; Shafti 2015). The studies by Poyurovsky reported enrolling first episode patients but the mean duration of illness in these studies ranged from three to six years (Poyurovsky 2003; Poyurovsky 2007). Seven studies recruited patients specifically with negative symptoms (Ganguli 2008; Hinkelmann 2013; Li 2008; Shafti 2015; Usall 2014; Yu 2012; Zhao 2013); and one recruited participants with depressive symptoms (Kurland 1981). Specific inclusion criteria included patients with metabolic syndrome (Zhao 2013) or with weight gain (Ball 2011). Overall baseline severity (as rated by mean or estimated CGI score) ranged from borderline mentally ill (Friedman 2008), mildly ill (Ball 2011; Ganguli 2008; Li 2008; Schutz 2001; Usall 2014; Yu 2012; Zhao 2013), moderately ill (Poyurovsky 2003; Poyurovsky 2007), markedly ill (Hinkelmann 2013; Kelly 2009), to severely ill (Shafti 2015).

Two studies involved only patients taking clozapine (Li 2008; Zhao 2013), with two further studies including a mixture of patients taking clozapine and other atypical antipsychotics (Ball 2011; Hinkelmann 2013). The majority of studies included participants taking a variety of atypical antipsychotics (Eli Lilly 2006; Friedman 2008; Kelly 2009; Poyurovsky 2003; Poyurovsky 2007; Usall 2014; Yu 2012); while three studies included participants taking only typical antipsychotics (Kurland 1981; Schutz 2001; Shafti 2015). 


\subsection{Study size}

There were 919 participants across all trials with 72 of these from the two studies which were only included in the qualitative discussions due to a lack of useable data (Ganguli 2008; Sacco 2009); and 39 from the citalopram arms of two trials (Hinkelmann 2013; Usall 2014). Most studies were small: two studies had 20 participants or fewer (Friedman 2008; Sacco 2009); six studies had 21 to 50 participants (Ball 2011; Kelly 2009; Kurland 1981; Poyurovsky 2003; Schutz 2001; Shafti 2015); five had 51 to 100 participants (Ganguli 2008; Hinkelmann 2013; Poyurovsky 2007; Usall 2014; Yu 2012); and three had more than 100 participants (Eli Lilly 2006; Li 2008; Zhao 2013).

\subsection{Interventions}

\subsubsection{Reboxetine}

Nine studies used reboxetine as the intervention. Most used up to 8 mg daily (Hinkelmann 2013; Li 2008; Schutz 2001; Usall 2014; Yu 2012; Zhao 2013) with the rest using up to $4 \mathrm{mg}$ daily (Poyurovsky 2003; Poyurovsky 2007; Shafti 2015).

\subsubsection{Atomoxetine}

Six studies used atomoxetine as the intervention. Most titrated up to 80 mg daily (Eli Lilly 2006; Friedman 2008; Ganguli 2008; Kelly 2009). One study used doses up to $120 \mathrm{mg}$ daily (Ball 2011); and there was one study with two arms which used $40 \mathrm{mg}$ and $80 \mathrm{mg}$ daily (Sacco 2009).

\subsubsection{Other drug treatment arms}

One study used viloxazine in doses up to $300 \mathrm{mg}$ daily (Kurland 1981). In two studies, in addition to the reboxetine and placebo arms, there was also a citalopram arm with one study using doses up to $30 \mathrm{mg}$ (Usall 2014) and one up to $40 \mathrm{mg}$ daily (Hinkelmann 2013).

\subsection{Funding}

Four studies did not report their source of funding (Li 2008; Schutz 2001; Yu 2012; Zhao 2013). Three studies were funded partly or wholly by Eli Lilly (Ball 2011; Eli Lilly 2006; Friedman 2008). Eli Lilly also contributed study medication to a further trial (Kelly 2009). Two studies received medication or assistance from other pharmaceutical companies (Kurland 1981; Poyurovsky 2003). Four studies were funded partly or wholly by US Government agencies such as the National Institutes of Health (NIH) (Ball 2011; Friedman 2008; Kelly 2009; Sacco 2009); and a further two trials received funding from other state sources outside the US (Shafti 2015; Usall 2014). The Stanley Medical Research Institute provided funding for four trials (Ganguli 2008; Hinkelmann 2013; Kelly 2009; Poyurovsky 2007); and the National Alliance for Research on Schizophrenia and Depression (NARSAD) contributed funding to one study (Sacco 2009).

\subsection{Outcomes}

None of the included studies reported results for service utilisation, satisfaction, or economic outcomes.

\subsubsection{Mental state}

\subsubsection{Positive and Negative Syndrome Scale (PANSS)}

The PANSS is a 30 -item structured clinical interview producing three subscales, positive (seven items), negative (seven items) and general psychopathology (such as anxiety or depressed mood) (16 items) with individual items rated one to seven (Kay 1986). Higher scores indicate more severe illness.

\subsubsection{Brief Psychiatric Rating Scale (BPRS)}

The BPRS is a clinician-rated instrument used to score 18 items of psychopathology (such as hallucinations or depressed mood) rated one to seven (Overall 1962). Higher scores indicate more severe illness.

\subsubsection{Scale for the Assessment of Negative Symptoms (SANS)}

The SANS is a 26-item clinician-rated scale measuring negative symptoms of schizophrenia across five domains (such as alogia or avolition) and items are rated from zero to five. Higher scores indicate more severe illness (Andreasen 1982).

\subsubsection{Scale for the Assessment of Positive Symptoms (SAPS)}

The SAPS is a 34-item clinician-rated scale measuring positive symptoms of schizophrenia across four domains (such as hallucinations or delusions) and items are rated from zero to five. Higher scores indicate more severe illness (Andreasen 1984).

\subsubsection{Hamilton Rating Scale for Depression (HRSD)}

The HRSD is a semi-structured interview producing a 17-item scale of depressive symptoms (such as depressed mood or feelings of guilt) with each item rated from zero to two, three, or four (depending on the item) for a maximum score of 53 (Hamilton 1980). Longer versions such as a 24-item scale have been developed but as far as we could determine all studies utilised the 17-item scale. Higher scores indicate more severe illness.

\subsubsection{Cognitive functioning}

As predicted, a wide-range of cognitive tests were utilised in the included studies. These have been classified according to the MATRICS-NIMH domains (Nuechterlein 2004) and individual tests are not discussed in detail.

\subsubsection{Clinical global response}

\subsubsection{Clinical Global Impression (CGI)}

\subsection{Clinical Global Impression - Severity (CGI-S)}

The CGI-S is a clinician-rated scale where the severity of the patient's illness is scored from one to seven based on clinical experience with higher scores indicating more severe illness (Guy 1976).

\subsection{Clinical Global Impression - Improvement (CGI-I)}

The CGI-I is a clinician-rated scale where the deterioration or improvement in the patient's illness compared to baseline is scored from one to seven where one indicates 'very much improved' and seven indicates 'very much worse' so higher scores indicate more severe illness (Guy 1976).

\subsubsection{Quality of life}

The General Quality of Life Inventory-74 (GQOLI-74) is based on the World Health Organization's Quality of Life Assessment Instrument modified for use in a Chinese population (Lu 2007). It is a 74-item inventory with 20 subscores in four domains. Higher scores indicate better quality of life. 


\subsubsection{Social functioning}

1.7.5.1 Scale of Social-skills for Psychiatric Inpatients (SSPI)

The SSPI is a published Chinese scale with ten factors of social functioning each from three items scored 0 to 2 (Guo 1995). Higher scores represent worse performance.

\subsubsection{Specific Level of Function (SLOF)}

The SLOF scale is a 43-item instrument administered to the patient's caregiver to assess functional performance in six domains (Schneider 1983). Items are scored on a five-point Likert scale. Higher scores indicate better performance.

\subsubsection{Adverse effects}

1.7.6.1 Abnormal Involuntary Movement Scale (AIMS)

The AIMS is a clinician-rated scale to assess the severity of tardive dyskinesia (Munetz 1988). The 12 items are scored zero to four with a higher score indicating worse side-effects.

\subsubsection{Simpson-Angus Scale (SAS)}

The SAS is a clinician-rated scale to assesses parkinsonism in schizophrenia (Simpson 1970). Ten items are scored zero to four, higher scores indicate worse side-effects.

\subsubsection{Barnes Akathisia Scale (BAS)}

The BAS is a clinician-rated scale of drug-induced akathisia incorporating subjective and objective ratings scored from zero to three with an additional six-point scale to assess global severity (Barnes 1989). Higher scores indicate worse side-effects.

\section{Excluded studies}

We excluded eight studies; details of individual studies are shown in Characteristics of excluded studies. Three excluded studies were of citalopram which is not a selective NRI and thus does not meet the inclusion criteria (Barnes 2009; Hou 2007; Salokangas 1997). The publication by Mueller 2005 reported on two randomised placebo-controlled add-on trials of a COX2-inhibitor, one trial examined patients with depression who were receiving reboxetine and another examined patients with schizophrenia who were receiving risperidone. Therefore it was not a trial of an NRI in schizophrenia. Shafti 2004 reported trials of multiple psychotropic medications including maprotiline. We did not regard any of these compounds as selective NRIs; in particular we excluded maprotiline due to its significant actions at multiple monoamine receptors in addition to noradrenaline reuptake inhibition.

Amrami-Weizman 2013 combined data from Poyurovsky 2003 and Poyurovsky 2007 to report metabolic and hormonal measures which were not defined as outcomes of interest in this review. Apud 2007a conducted a cross-over RCT of atomoxetine in schizophrenia stratified by COMT genotype: only four patients completed the trial and no data was collected. It was terminated early due to slow recruitment and as a low scientific priority for NIMH. Poyurovsky 2013 reported an RCT of the combination of reboxetine and betahistine in schizophrenia; therefore betahistine use was not equally distributed across treatment and control arms and the study did not meet inclusion criteria. This study found a benefit of the reboxetine-betahistine combination on reducing weight gain from olanzapine but no effect on symptom outcome measures such as the SANS.

\section{Risk of bias in included studies}

Information for risk of bias across the included studies is illustrated in Figure 2 and Figure 3. There were three incidents of nonconcurrence in risk ratings: two for assessment of blinding (Friedman 2008; Kurland 1981); and one for assessment of selection bias (Shafti 2015). All decisions were between 'unclear risk' and 'low risk' and overall ratings of 'unclear risk' were agreed in all three cases. The overall value of a weighted kappa was high at 0.96 for 112 decisions across 16 trials.

Figure 2. Risk of bias graph: review authors' judgements about each risk of bias item presented as percentages across all included studies.

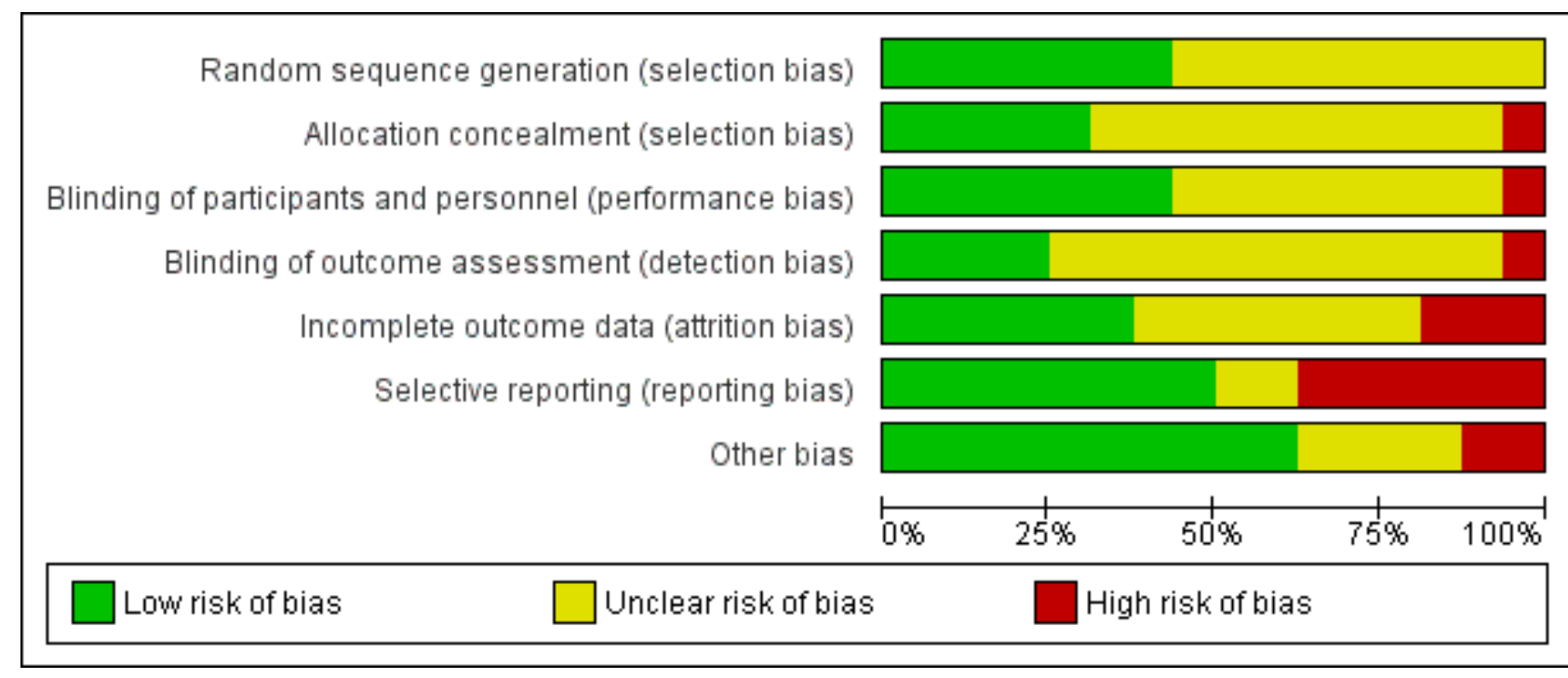


Figure 3. Risk of bias summary: review authors' judgements about each risk of bias item for each included study.

\begin{tabular}{|c|c|c|c|c|c|c|c|}
\hline & 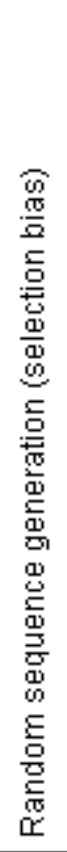 & 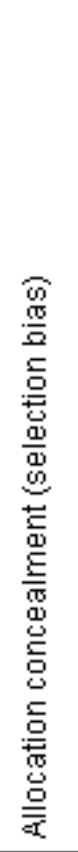 & 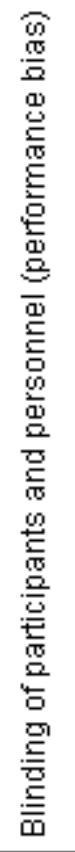 & 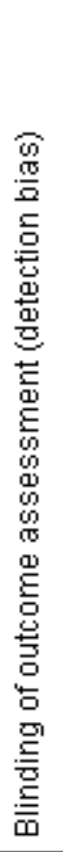 & 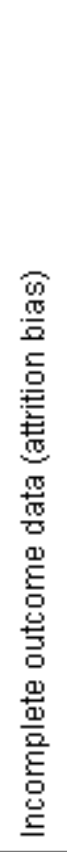 & 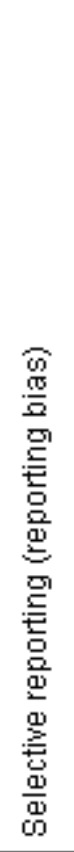 & 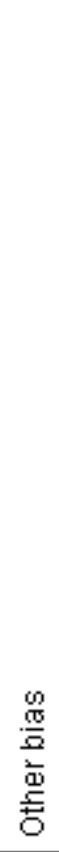 \\
\hline Ball 2011 & + & + & + & $?$ & $\odot$ & - & + \\
\hline Eli Lilly 2006 & $?$ & $?$ & $?$ & $?$ & - & - & $?$ \\
\hline Friedman 2008 & $?$ & $?$ & $?$ & $?$ & + & $\oplus$ & + \\
\hline Ganguli 2008 & $?$ & $?$ & $?$ & $?$ & - & - & $?$ \\
\hline Hinkelmann 2013 & + & + & $?$ & $?$ & $?$ & $?$ & + \\
\hline Kelly 2009 & 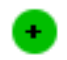 & $\odot$ & $\odot$ & $?$ & $?$ & $\odot$ & - \\
\hline Kurland 1981 & $?$ & $?$ & $?$ & $?$ & $\oplus$ & & $?$ \\
\hline Li 2008 & $?$ & $?$ & $\odot$ & $?$ & $\odot$ & $?$ & + \\
\hline Poyurovsky 2003 & 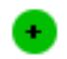 & $?$ & $?$ & $\odot$ & $\oplus$ & $\odot$ & + \\
\hline Poyurovsky 2007 & + & $?$ & + & $\odot$ & $\odot$ & $\odot$ & + \\
\hline Sacco 2009 & $?$ & $?$ & $?$ & $?$ & $?$ & - & $?$ \\
\hline Schut 2001 & $\odot$ & $\odot$ & + & ? & ? & $\odot$ & + \\
\hline Shafti 2015 & $?$ & $?$ & $\odot$ & $\odot$ & $\odot$ & $\odot$ & + \\
\hline Usall 2014 & + & $\odot$ & $\odot$ & $\odot$ & $?$ & $\odot$ & + \\
\hline Yu 2012 & $?$ & - & - & - & $?$ & - & - \\
\hline Zhao 2013 & $?$ & $?$ & $?$ & $?$ & $?$ & $\odot$ & + \\
\hline
\end{tabular}




\section{Allocation}

All studies were described as randomised but only seven studies reported the method of randomisation and were rated as low risk (Ball 2011; Hinkelmann 2013; Kelly 2009; Poyurovsky 2003; Poyurovsky 2007; Schutz 2001; Usall 2014). One study was described as open and was considered high risk for allocation (Yu 2012). The remaining studies were described as double-blind and placebo-controlled although only five studies described the method of allocation concealment in detail and were rated as low risk (Ball 2011; Hinkelmann 2013; Kelly 2009; Schutz 2001; Usall 2014). The remaining studies were rated as unclear risk.

\section{Blinding}

The majority of studies did not report blinding in detail but we considered the one open study (Yu 2012) to be at high risk for inadequate blinding. Seven studies described blinding of participants and we considered them low risk (Ball 2011; Kelly 2009; Li 2008; Poyurovsky 2007; Schutz 2001; Shafti 2015; Usall 2014). Four studies described blinding of outcomes and we rated them as low risk (Poyurovsky 2003; Poyurovsky 2007; Shafti 2015; Usall 2014). We rated the remaining studies as unclear risk.

\section{Incomplete outcome data}

Six studies accounted for incomplete data and we considered them low risk (Friedman 2008; Kurland 1981; Li 2008; Poyurovsky 2003; Poyurovsky 2007; Shafti 2015); while three were classified as high risk due to a very high rate of withdrawals (Ganguli 2008) or an imbalance in withdrawals between arms (Ball 2011; Eli Lilly 2006). We rated the remaining studies as unclear risk.

\section{Selective reporting}

Eight studies appeared to report all relevant outcomes and were rated as low risk (Friedman 2008; Kelly 2009; Poyurovsky 2003; Poyurovsky 2007; Schutz 2001; Shafti 2015; Usall 2014; Zhao 2013). Six studies demonstrated selective reporting of outcomes (Ball 2011; Eli Lilly 2006; Ganguli 2008; Kurland 1981; Sacco 2009; Yu 2012). The remaining studies were rated as unclear risk.

\section{Other potential sources of bias}

The majority of studies did not demonstrate evidence of other sources of bias and we rated them as low risk. We gave two studies a high risk of bias due to excluding participants after randomisation (Kelly 2009), and evidence of methodological flaws (such as mean PANSS total scores < 30) (Yu 2012). For four studies we rated them as having an unclear risk of bias due to methodological flaws as it was not clear that these would have a significant impact on the results (Eli Lilly 2006; Ganguli 2008; Kurland 1981; Sacco 2009).

\section{Effects of interventions}

See: Summary of findings for the main comparison Noradrenaline reuptake inhibitors versus placebo

Two studies did not report any outcomes that could be used in the meta-analysis and are included in the narrative review only (Ganguli 2008; Sacco 2009). As far as could be determined all studies included patients with a duration of illness of six months or more with the majority over one year, with many described as stable or chronic; and as we did not perform sensitivity analysis looking at duration or stage of illness except to consider studies including participants taking clozapine or recruiting specifically participants with prominent negative symptoms.

Under each heading of the Summary of findings for the main comparison we included the analysis with the longest period of follow-up (providing there were more than three studies to include for that time period), otherwise we used the duration which included the most studies. Where there was more than one outcome measure (e.g. PANSS and BPRS) for a time-point we used the outcome with the most studies.

\section{Comparison 1. NRI versus placebo}

\subsection{Primary outcome - A. Mental state: Specific - clinically significant response or improvement in negative symptoms (SANS, high = worse) - short term (12 weeks)}

While all 16 studies included in the review utilised instruments which could produce an indication of rates of improvement in negative symptoms only one provided usable data. Shafti 2015 measured response rates at 12 weeks (defined as $20 \%$ reduction in the SANS) and reported rates of $6 / 25$ and $19 / 25$ in the placebo and reboxetine arms respectively indicating a significant benefit of reboxetine ( $1 \mathrm{RCT}, \mathrm{n}=50$; RR 3.17, $95 \% \mathrm{Cl} 1.52$ to 6.58 ; Analysis 1.1).

\subsection{Primary outcome - B. Clinical global response: clinically significant response or improvement in global status (CGI-S, high $=$ worse) - short term (4 weeks)}

Kurland 1981 reported non-specific improvement rates of 7/15 and $6 / 13$ in the placebo and viloxazine arms respectively, showing no difference (Analysis 1.2). Nine studies measured global response using the CGI-I or CGI-S but none defined clinical improvement on these scales or reported dichotomous outcomes.

\subsection{Mental state: 1. General - clinically significant response or improvement in general/overall symptoms as defined in each study - short term (2 to 12 weeks)}

Kurland 1981 reported non-specific improvement rates of 7/15 and $6 / 13$ in the placebo and violoxazine arms respectively at 4 weeks (these data have also been included under the primary outcome measure of clinical global response), while Schutz 2001 reported response rates at 6 weeks (defined as 20\% reduction in PANSS total score) and we calculated ITT rates of $5 / 15$ and $7 / 15$ for placebo and reboxetine respectively. When combined $(n=58)$ we found no significant benefit of NRIs (RR 1.15, 95\% Cl 0.64 to 2.09; Analysis 1.3).

\subsection{Mental state: $2 a$. General: average general/overall symptoms score (various scales, high $=$ worse) - short term (2 to 12 weeks)}

Nine studies reported a useable overall clinical symptom scale or general subscale score from either the PANSS or the BPRS.

Li 2008 reported PANSS total and general endpoint scores at 12 weeks. Yu 2012 reported PANSS total endpoint scores at 8 weeks. Zhao 2013 reported PANSS total and general endpoint scores at 8 weeks. Friedman 2008 reported completer-only PANSS general change scores at 8 weeks and we calculated LOCF PANSS general endpoint scores with an estimated SD but these were skewed and entered into Analysis 1.8. Hinkelmann 2013 reported LOCF PANSS general endpoint scores at 4 weeks which were skewed and entered into Analysis 1.8 and LOCF PANSS general change scores were calculated with an estimated SD. Kelly 2009 reported completer- 
only BPRS total scores at 8 weeks which were skewed and entered into Analysis 1.8 and completer-only BPRS change scores were calculated with an estimated SD. Schutz 2001 reported PANSS total and general endpoint scores at 6 weeks which were skewed and entered into Analysis 1.8 and we calculated change scores and estimated the SD. Usall 2014 reported PANSS total and general endpoint scores at 24 weeks which were skewed and entered into Analysis 1.8 and we calculated change scores and estimated the SD.

\subsubsection{BPRS total}

Kelly 2009 reported BPRS total change scores and found no benefit of atomoxetine at 8 weeks (MD 1.50, 95\% Cl -6.64 to 9.64; Analysis 1.4).

\subsubsection{PANSS general}

Five studies had results for the PANSS general subscale $(n=294)$ with a statistically significant benefit of NRIs (MD $-2.17,95 \% \mathrm{Cl}$ -3.93 to -0.40 ; Analysis 1.4 ).

We performed subgroup and sensitivity analysis (see Table 1). The only study of atomoxetine is Friedman 2008, which shows no benefit; and excluding this to look only at reboxetine studies makes little difference. The two studies looking at only patients on clozapine were both positive (MD $-2.8 ; 95 \% \mathrm{Cl}-4.87$ to -0.72 ) (Li 2008; Zhao 2013); and looking only at studies that included patients taking clozapine, which were the studies that specifically recruited patients with negative symptoms, made little difference to the outcome (Hinkelmann 2013; Li 2008; Zhao 2013). The change scores in Hinkelmann 2013 and Schutz 2001 have imputed SDs and excluding these slightly inflated the effect size (MD -2.66, 95\% $\mathrm{Cl}-4.50$ to -0.82 ). Excluding all change scores associated with a skewed endscore (Friedman 2008; Hinkelmann 2013; Schutz 2001) left the two clozapine-only studies utilising unskewed endpoint scores (Li 2008; Zhao 2013). A fixed-effect model made little difference except to slightly inflate the effect size and narrow the confidence intervals (MD $-2.73,95 \% \mathrm{Cl}-3.71$ to -1.74 ). No studies were at high risk of bias for randomisation but only two studies described their method in any detail (Hinkelmann 2013; Schutz 2001), and combining these showed no benefit for NRIs (MD 0.89, $95 \% \mathrm{Cl}-3.60$ to 5.37 ). No studies were at high risk of bias in any domain.

\subsubsection{PANSS total}

Four studies had short-term PANSS total score results $(\mathrm{n}=309)$ which showed a significant benefit of reboxetine (MD -2.84, 95\% $\mathrm{Cl}-5.28$ to -0.40 ; Analysis 1.4$)$ and substantial heterogeneity $\left(\mathrm{I}^{2}=\right.$ $72 \%)$.

We performed subgroup and sensitivity analysis (see Table 2). Visually there was no clear outlying trial contributing to heterogeneity but excluding the open study by Yu 2012 abolished the heterogeneity $\left(I^{2}=0 \%\right)$ while magnifying the effect size (MD $-4.20,95 \% \mathrm{Cl}-5.82$ to -2.58$)$. The two studies of patients taking only clozapine both showed a significant benefit of reboxetine (MD $-4.25,95 \% \mathrm{Cl}-5.89$ to -2.62 ) (Li 2008; Zhao 2013). Excluding Schutz 2001 , the single study that did not specifically recruit patients with negative symptoms, made little difference to the overall effect. Schutz 2001 was also the only study using imputed SD. A fixedeffect model made marginal difference to the overall outcome. No study was considered to be at high risk of bias for randomisation but only Schutz 2001 described the method of randomisation in detail. Yu 2012 was considered at high risk of bias for allocation concealment, blinding of participants and outcomes, selective reporting, and other sources of bias.

\subsection{Mental state: $2 b$. General: average general/overall symptoms score (various scales, high $=$ worse) - medium term (13 to 26 weeks)}

Yu 2012 reported PANSS total endpoint scores at 16 weeks but we considered these to be skewed, partially because the mean scores were less than 30 which should not be possible with the PANSS total scale, and this was entered into Analysis 1.9. We calculated change scores at 16 weeks and estimated the SD. Zhao 2013 reported PANSS total and general endpoint scores at 24 weeks. Ball 2011 reported BPRS total endpoint scores at 24 weeks which were skewed and are entered into Analysis 1.9 and not included in the meta-analysis but change scores were calculated with an estimated SD. Usall 2014 reported PANSS total and general endpoint scores at 24 weeks which were skewed and entered into Analysis 1.9 and we calculated change scores and estimated SD.

\subsubsection{BPRS total}

Ball 2011 reported BPRS total change scores and found no benefit of atomoxetine at 24 weeks (MD $-1.40,95 \% \mathrm{Cl}-7.08$ to 4.28 ; Analysis 1.5).

\subsubsection{PANSS general}

Usall 2014 and Zhao 2013 reported the PANSS general subscale for reboxetine and these were not consistent, with the former showing no effect and the latter a large benefit for reboxetine resulting in a combined estimate that was not significant (MD $-2.90,95 \% \mathrm{Cl}-7.57$ to 1.77 ; Analysis 1.5).

\subsubsection{PANSS total}

Three studies reported this outcome $(n=219)$ with a large benefit of reboxetine without statistical significance (MD $-3.67,95 \% \mathrm{Cl}-10.07$ to 2.72; Analysis 1.5) and substantial heterogeneity $\left(I^{2}=94 \%\right)$.

There were only three trials for medium-term outcomes so we did not perform a full subgroup and sensitivity analysis but we did look for sources of heterogeneity. The large study by Zhao 2013, which was the only one to look at patients taking clozapine, showed a much greater benefit of reboxetine than the other two studies and could be considered an outlier visually: excluding this both abolished heterogeneity and attenuated the benefit of reboxetine (MD $-1.07,95 \% \mathrm{Cl}-2.59$ to 0.46$)$. A fixed-effect model makes little difference to the estimated effect size but narrows the confidence intervals to become statistically significant (see Table 3).

1.6 Mental state: 3a. Specific: average symptoms score (various scales, high $=$ worse) - short term (2 to 12 weeks)

\subsection{1 mood (BPRS)}

Kelly 2009 reported BPRS anxiety/depression subscale endpoint completer scores at 8 weeks but these were skewed. We entered them into Analysis 1.8 and calculated change scores and estimated SD.

There was no difference between atomoxetine and placebo (MD $0.20,95 \% \mathrm{Cl}-2.60$ to 3.00 ; Analysis 1.6). 


\subsection{2 mood (HRSD)}

Hinkelmann 2013 reported LOCF HRSD endpoint scores at 4 weeks but these were skewed and entered into Analysis 1.8 and we calculated change scores and imputed the SD. Poyurovsky 2003 reported endpoint scores at 6 weeks but these were skewed and entered into Analysis 1.8 and we calculated change scores and imputed the SD. Poyurovsky 2007 reported endpoint scores at 6 weeks from cognitive testing completers only but these were skewed and entered into Analysis 1.8. They also reported ITT change scores. Schutz 2001 reported endpoint scores for completers at 6 weeks but these were skewed and entered into Analysis 1.8. We could not calculate change scores but there was no difference between the groups in mean scores.

Combining the three studies $(n=114)$ reporting change scores for the HRSD showed a significant benefit of reboxetine (MD -2.37 , $95 \% \mathrm{Cl}-4.29$ to -0.45 ; Analysis 1.6$)$. As there were only three trials we did not perform a full subgroup and sensitivity analysis but a fixed-effect model made little difference (MD $-2.53,95 \% \mathrm{Cl}-4.03$ to $-1.03)$.

\subsection{3 negative (PANSS negative)}

Friedman 2008 reported observed PANSS negative change scores at 8 weeks and we calculated LOCF endpoint scores with imputed SD which were skewed and entered into Analysis 1.8. Hinkelmann 2013 reported LOCF end point scores at 4 weeks which were skewed and entered into Analysis 1.8 and we calculated change scores with imputed SD. Li 2008 reported endpoint scores at 12 weeks. Schutz 2001 reported endpoint scores for completers at 6 weeks which were skewed and entered into Analysis 1.8 and we derived change scores with imputed SD. Yu 2012 and Zhao 2013 reported endpoint scores at 8 weeks.

Together, these six $(n=359)$ studies showed no statistically significant benefit of NRIS (MD $-0.99,95 \% \mathrm{Cl}-2.53$ to 0.56 ; Analysis 1.6) with significant heterogeneity $\left(I^{2}=71 \%\right)$.

We performed subgroup and sensitivity analysis (see Table 4). Visually there was no clear individual outlier and excluding any individual study did not markedly reduce the heterogeneity. Friedman 2008 was the only study reporting outcomes for atomoxetine and this showed no benefit. Excluding Friedman 2008 to analyse the effect of reboxetine slightly increased the overall benefit which remained non-significant (MD $-1.20,95 \% \mathrm{Cl}-2.80$ to 0.40). The two studies that enrolled only patients on clozapine were inconsistent but magnified the benefit when combined, although this remained non-significant (MD $-1.60,95 \% \mathrm{Cl}-3.96$ to 0.76 ) (Li 2008; Zhao 2013). Adding Hinkelmann 2013 (which included some patients on clozapine) attenuated the overall effect further (MD $-0.75,95 \% \mathrm{Cl}-3.25$ to 1.75 ). Four trials specifically recruited patients with negative symptoms which inflated the benefit of NRIs but remained non-significant (MD $-1.47,95 \% \mathrm{Cl}-3.04$ to 0.09 ).

Only three studies did not have associated skew and combining these inflated the benefit of reboxetine which became statistically significant (MD -1.92; 95\% Cl -3.28 to -0.55) (Li 2008; Yu 2012; Zhao 2013). We imputed the SD for two studies and excluding these inflated the benefit of reboxetine which became statistically significant (MD -1.68, 95\% Cl -3.04 to -0.32 ) (Hinkelmann 2013; Schutz 2001). Using a fixed-effect model inflated the benefit of NRIs which became statistically significant (MD $-1.82,95 \% \mathrm{Cl}-2.46$ to $-1.18)$.
We did not determine that any of these studies were at high risk of bias for randomisation. Only two studies described their randomisation methods in any detail and we considered these at low risk of bias. Combining these two studies showed a large nonsignificant benefit for placebo over reboxetine (MD 3.55, 95\% Cl -0.43 to 7.54) (Hinkelmann 2013; Schutz 2001). We classified Yu 2012 as high risk of bias for allocation concealment, blinding of participants and outcomes, selective reporting, and other sources of bias and excluding this study largely abolished the benefit of NRIs (MD $-0.12,95 \% \mathrm{Cl}-2.31$ to 2.08 ). Most sensitivity analyses made little impact on the high overall heterogeneity.

\subsection{4 negative (SANS)}

Poyurovsky 2003 reported SANS endpoint scores at 6 weeks which were skewed and entered into Analysis 1.8 and we calculated change scores with estimated SD. Poyurovsky 2007 reported ITT change scores at 6 weeks and we derived endpoint data and imputed SD. Shafti 2015 reported endpoint scores at 12 weeks.

Combined together these three $(n=129)$ studies showed a nonsignificant benefit of reboxetine (MD $-2.47,95 \% \mathrm{Cl}-6.22$ to 1.28 ; Analysis 1.6) and substantial heterogeneity $\left(\mathrm{I}^{2}=71 \%\right)$.

As there were only three trials we did not perform a full subgroup and sensitivity analysis but we looked for sources of heterogeneity. There was no one clear outlier when we inspected visually. A fixedeffect model attenuates the effect size but becomes statistically significant (MD -1.66, 95\% $\mathrm{Cl}-3.09$ to -0.22 ).

\subsection{5 negative (SANS - modified)}

Kelly 2009 reported modified SANS endpoint completer scores at 8 weeks. As this has been modified from the original SANS instrument it was not combined directly with other SANS results.

This showed a large benefit of atomoxetine that was not statistically significant (MD $-5.70,95 \% \mathrm{Cl}-18.01$ to 6.61 ; Analysis 1.6).

\subsection{6 positive (BPRS)}

Kelly 2009 reported completer BPRS positive endscores at 8 weeks which were skewed and entered into Analysis 1.8 and we calculated change scores and imputed SD.

This study produced a non-significant benefit for placebo over atomoxetine (MD 1.60, 95\% Cl-2.59 to 5.79; Analysis 1.6).

\subsection{7 positive (PANSS positive)}

Friedman 2008 reported PANSS positive change scores for completers at 8 weeks and we calculated endpoint LOCF scores with imputed SDs which were skewed and we entered these into Analysis 1.8. Hinkelmann 2013 reported LOCF endscores at 4 weeks but these were skewed and we entered them into Analysis 1.8 calculating change scores with estimated SD. Li 2008 report endscores at 12 weeks which were skewed and entered into Analysis 1.8 and we calculated change scores and estimated the SD. Schutz 2001 report endscores for completers at 6 weeks which were skewed and entered into Analysis 1.8 and we derived change scores with estimated SD. Zhao 2013 report endscores at 8 weeks which were skewed and entered into Analysis 1.8 and we calculated change scores with SD imputed.

Five studies $(n=294)$ reported outcomes with no benefit or harm from NRIs (MD $-0.16,95 \% \mathrm{Cl}-0.96$ to 0.63 ; Analysis 1.6). 
We performed subgroup and sensitivity analysis for short-term outcomes (see Table 5). There was only one study of atomoxetine which showed a non-significant tendency to favour placebo (Friedman 2008); and looking only at studies of reboxetine made little difference to the outcome. The two studies looking at patients taking clozapine only did not show a benefit of reboxetine ( $\mathrm{Li}$ 2008; Zhao 2013); and looking only at those studies including patients on clozapine (Hinkelmann 2013; Li 2008; Zhao 2013), which were also the studies recruiting patients specifically with negative symptoms, made minimal difference. All studies had imputed SDs except Friedman 2008 and all change scores were associated with skewed endscores. A fixed-effect model made no difference. We determined that none of the studies were at high risk of bias for randomisation but only two studies reported these methods in any detail and they did not show a benefit for reboxetine (Hinkelmann 2013; Schutz 2001). We did not classify any studies at high risk of bias for any domain.

\subsection{8 positive (SAPS)}

Poyurovsky 2003 report SAPS endscores at 6 weeks which are skewed and entered into Analysis 1.11 and we calculated the change scores and imputed the SD. Poyurovsky 2007 report ITT change scores at 6 weeks and we derived endscores using an imputed SD which were skewed and entered into Analysis 1.11. Shafti 2015 report endscores at 12 weeks.

We combined three studies $(n=129)$ which showed no overall benefit from reboxetine (MD $0.73,95 \% \mathrm{Cl}-1.29$ to 2.74; Analysis 1.6). There were only three trials so a full subgroup and sensitivity analysis was not performed but a fixed-effect model made little difference (MD 0.59, 95\% $\mathrm{Cl}-1.16$ to 2.33 ).

\subsection{Mental state: $3 b$. Specific: Average symptoms score (various} scales, high $=$ worse) - medium term (13 to 26 weeks)

\subsection{1 negative (PANSS negative)}

Usall 2014 reported PANSS negative endpoint scores at 24 weeks which were skewed and entered into Analysis 1.9 and we calculated change scores with imputed SDs. Yu 2012 reported endpoint scores at 16 weeks. Zhao 2013 reported endpoint scores at 24 weeks.

These three studies $(n=219)$ showed a significant benefit for reboxetine (MD $-3.25,95 \% \mathrm{Cl}-4.04$ to -2.47 ; Analysis 1.7 ) without heterogeneity.

There were only three trials so we did not perform a full subgroup and sensitivity analysis but a fixed-effect model made no difference to the result (MD $-3.25,95 \% \mathrm{Cl}-4.04$ to -2.47 ).

\subsection{2 negative (SANS)}

Usall 2014 reported SANS endpoint scores at 24 weeks which were skewed and entered into Analysis 1.9 and we calculated change scores with imputed SDs.

This showed a large benefit for reboxetine that was not significant (MD -7.12, 95\% Cl-19.39 to 5.15; Analysis 1.7)

\subsection{3 positive (PANSS positive)}

Usall 2014 reported PANSS positive endscores at 24 weeks which were skewed and entered into Analysis 1.9 with change scores calculated and SD imputed. Zhao 2013 report endscores at 24 weeks which were skewed and entered into Analysis 1.9 with change scores calculated and SD imputed.

The reboxetine studies by Usall 2014 and Zhao 2013 showed no benefit overall (MD $-0.14,95 \% \mathrm{Cl}-1.30$ to 1.02 ; Analysis 1.7).

\subsection{Mental state: 4a. General and specific: Average score (various scales, high = worse) - skewed results - short term (2 - 12 weeks)}

Data for this outcome were skewed and are presented as 'other data' .

\subsection{Mental state: 4b. General and specific: Average score (various scales, high = worse) - skewed results - medium term (13 - 26 weeks)}

Data for this outcome were skewed and are presented as 'other data' .

\subsection{Cognitive functioning: 1. General - average composite cognitive functioning score (SMD)}

\subsection{1 short term ( 2 to 12 weeks)}

Eli Lilly 2006 reported a LOCF composite cognitive change score at 8 weeks. Friedman 2008 reported composite cognitive change scores for completers at 8 weeks. Kelly 2009 reported composite cognitive endscores and change scores for completers at 8 weeks. Poyurovsky 2007 did not report composite cognitive scores but we were able to calculate a composite score at 6 weeks using mean SMD from individual cognitive scale endscores (excluding those that were skewed) with estimated SD.

When combined these four $(n=180)$ studies showed no overall benefit from NRIs (SMD 0.04, $95 \% \mathrm{Cl}-0.28$ to 0.36 ; Analysis 1.10 ).

We performed subgroup and sensitivity analysis for short-term outcomes (see Table 6). There was only one reboxetine trial which showed no benefit and this was the one study where we calculated the composite effect size ourselves (Poyurovsky 2007). Excluding this to look only at studies of atomoxetine also showed no benefit. No study specifically recruited patients with negative symptoms. Using a fixed-effect model made little difference. No study was at high risk of bias for randomisation but only Kelly 2009 and Poyurovsky 2007 reported their methods in detail and their results were not consistent but combined they showed a non-significant benefit for NRIs (MD $-0.18,95 \% \mathrm{Cl}-1.06$ to 0.69 ). Two studies were considered at high risk for other sources of bias and excluding them showed a slight benefit for placebo (MD 0.14, 95\% - 0.42 to 0.71 ) (Eli Lilly 2006; Kelly 2009); while excluding only Eli Lilly 2006 which was also considered at high risk of attrition and reporting bias, had little effect.

\subsection{2 medium term ( 13 to 26 weeks)}

Ball 2011 reported a composite cognitive change score at 24 weeks.

This showed a large benefit of atomoxetine that was not statistically significant (SMD $-0.66,95 \% \mathrm{Cl}-1.46$ to 0.13 ; Analysis 1.16 ). 


\subsection{Cognitive functioning: 2. Specific - average individual scores (SMD) - short term (2 to 12 weeks)}

\subsection{1 working memory}

Kelly 2009 reported endscores from three working memory scales (WAIS-III letter-number sequencing, Woodcock-Johnson planning test, number sequencing test) for atomoxetine completers at 8 weeks and we used these to calculate a composite working memory SMD. Poyurovsky 2007 reported endscores from two working memory scales (matching to sample, mental rotation) with reaction time and percentage correct scores for each at 6 weeks for reboxetine. Mental rotation reaction times were skewed and we calculated a composite working memory SMD excluding this.

These showed little benefit of NRIs when combined (MD - 0.11, 95\% $\mathrm{Cl}-0.89$ to 0.67 ; Analysis 1.11) and a fixed-effect model made little difference (MD $-0.06,95 \% \mathrm{Cl}-0.60$ to 0.49 ).

\subsection{2 reasoning/problem solving}

Eli Lilly 2006 reported ITT change scores from the Tower of London test at 8 weeks. Friedman 2008 reported change scores from the Tower of London test at 8 weeks for completers. Poyurovsky 2007 reported endpoint scores from the Wisconsin card sorting task at 6 weeks with categories and percentage perseverative errors but the latter were skewed and we excluded them.

The three studies $(n=158)$ showed no benefit of NRIs (SMD 0.03, $95 \% \mathrm{Cl}-0.28$ to 0.34 ; Analysis 1.11 ). There were only three trials so we did not perform a full subgroup and sensitivity analysis but a fixed-effect model made no difference (MD $0.03,95 \% \mathrm{Cl}-0.28$ to 0.34).

\subsection{3 speed of processing}

Eli Lilly 2006 reported ITT change scores from three speed of processing measures (token motor task, verbal fluency, symbol coding task) at 8 weeks and we used these to calculate a composite speed of processing SMD. Friedman 2008 reported change scores from four speed of processing measures (token motor task, category instances test, controlled oral word association test, symbol coding task) at 8 weeks for completers and we used these to calculate a composite speed of processing SMD. Kelly 2009 reported completer only endscores from three speed of processing measures (WAIS-III digit symbol, grooved pegboard, letter fluency) at 8 weeks and we used these to calculate a composite speed of processing SMD. Poyurovsky 2007 reported endpoint scores from three speed of processing tasks (simple reaction time, code substitution, code substitution immediate recall) with reaction time and percentage correct scores for each at 6 weeks. We used these to calculate a composite speed of processing SMD although the simple reaction time task was excluded due to skew and $100 \%$ correct responses.

These four studies $(n=177)$ showed no benefit of NRIs (SMD $0.08,95 \% \mathrm{Cl}-0.21$ to 0.38 ; Analysis 1.11 ). We performed subgroup and sensitivity analysis (see Table 7). The one study of reboxetine showed a non-significant benefit of placebo (Poyurovsky 2007); and excluding this to look only at studies of atomoxetine did not change the overall lack of benefit of NRIs. We used calculated composite scores for all studies. None of the trials included patients on clozapine or recruited specifically those with negative symptoms. A fixed-effect model made no difference. No study was at high risk of bias for randomisation but only Kelly 2009 and Poyurovsky 2007 reported their methods in detail and their results were not consistent but overall suggested a small non-significant benefit for placebo (SMD $0.13,95 \% \mathrm{Cl}-0.41$ to 0.68 ). We considered two trials at high risk for attrition and other sources of bias and excluding these showed a non-significant benefit of placebo (SMD 0.23, 95\% $\mathrm{Cl}-.34$ to 0.80 ) (Eli Lilly 2006; Kelly 2009); while excluding just Eli Lilly 2006 , which was also considered at high risk of reporting bias, had little effect.

\subsection{4 attention}

Eli Lilly 2006 reported ITT change scores from the continuous performance test at 8 weeks. Kelly 2009 reported completer endscores for the GDS continuous performance distractibility test at 8 weeks. Poyurovsky 2007 reported endpoint scores from the continuous performance test at 6 weeks with reaction time and percentage correct scores combined to calculate a composite attention SMD.

These three studies $(n=161)$ showed no benefit of NRIs (SMD - 0.01 , $95 \% \mathrm{Cl}-0.51$ to 0.48 ; Analysis 1.11 ). There were only three trials so we did not perform a full subgroup and sensitivity analysis. A fixedeffect model favoured placebo but was not statistically significant (SMD $0.10,95 \% \mathrm{Cl}-0.21$ to 0.41 ).

\subsection{5 verbal learning/memory}

Eli Lilly 2006 reported ITT change scores for two verbal learning/ memory scales (verbal memory, digit sequencing) at 8 weeks and we tcombined these into a composite verbal learning/memory SMD. Friedman 2008 reported completer change scores for two verbal learning/memory measures (list learning, digit sequencing) at 8 weeks and these were combined into a composite verbal learning/memory SMD. Kelly 2009 reported completer endscores for the California verbal learning test at 8 weeks. Poyurovsky 2007 reported endpoint scores for the code substitution delayed recall task at 6 weeks with reaction time and percentage correct scores combined to calculate a composite verbal learning/memory SMD.

These four studies $(n=181)$ showed no benefit of NRIS (SMD $0.01,95 \% \mathrm{Cl}-0.31$ to 0.32 ; Analysis 1.11 ). Subgroup and sensitivity analysis was performed (see Table 8). Only Poyurovsky 2007 studied reboxetine which showed a non-significant benefit of placebo and excluding this made little difference (SMD $-0.08,95 \% \mathrm{Cl}-0.40$ to 0.25 ). None of the studies included patients on clozapine or recruited specifically with negative symptoms. Only Kelly 2009 did not use a composite score and this showed a large but nonsignificant benefit of atomoxetine. Using a fixed-effect model made little difference (SMD $0.01,95 \% \mathrm{Cl}-0.28$ to 0.30 ). We did not classify any study as high risk of bias for randomisation but only Kelly 2009 and Poyurovsky 2007 reported their methods in enough detail to be considered low risk and they showed no benefit when combined (SMD $-0.06,95 \% \mathrm{Cl}-1.04$ to 0.93 ). We determined that two studies were high risk for other sources of bias (Eli Lilly 2006; Kelly 2009); and excluding them inflated the benefit of placebo (SMD 0.26, $95 \% \mathrm{Cl}-0.31$ to 0.83 ). Eli Lilly 2006 was also considered at high risk of attrition and reporting bias and excluding this made little difference.

\subsection{6 visual learning/memory}

Kelly 2009 reported completer endscores for the brief visuospatial memory test at 8 weeks with a benefit for atomoxetine that was not statistically significant (SMD $-0.44 ; 95 \% \mathrm{Cl}-1.29$ to 0.41 ; Analysis 1.11). 
1.12 Quality of life: Average quality of life score (GQOLI-74, high = better) - short term (2 to 12 weeks)

The reboxetine study by Li 2008 reports GQOLI-74 endpoint total scores as well as subscores from four domains of functioning and 20 individual subscales at 12 weeks. We made a post hoc decision to consider the total score but also to report scores from the four domains. We did not report the individual subscales due to the likelihood of a small number of statistically significant differences due to chance and the view that a benefit of NRIs on any single subscale is unlikely to be of clinical relevance.

\subsection{1 general - total}

The GQOLI-74 total score (MD 9.36, 95\% Cl 7.89 to 10.83 ; Analysis 1.12) showed a significant benefit for reboxetine.

\subsection{2 specific - well-being - material}

The dimension of material well-being (MD $0.21,95 \% \mathrm{Cl}-2.34$ to 2.76; $N=114$ ) did not show a benefit (Analysis 1.12).

\subsection{3 specific - well-being - physical}

The dimension of physical well-being (MD $0.68,95 \% \mathrm{Cl}-1.35$ to 2.71 ; $\mathrm{N}=114$ ) did not show a benefit (Analysis 1.12).

\subsection{4 specific - well-being - psychological}

The dimension of psychological well-being (MD 10.00, 95\% Cl 8.01 to $11.99 ; \mathrm{N}=114$ ) showed a large benefit for reboxetine over placebo (Analysis 1.12).

\subsection{5 specific - well-being - social}

The dimension of social well-being (MD $10.02,95 \% \mathrm{Cl} 8.03$ to $12.01 ; \mathrm{N}=114$ ) showed a large benefit for reboxetine over placebo (Analysis 1.12).

\subsection{Clinical global response: 1a. Average clinical global status score (CGI-S, high = worse) - short term (2 to 12 weeks)}

Hinkelmann 2013 reported CGI-S endscores at 4 weeks which were skewed and entered into Analysis 1.14 and we estimated change scores with imputed SD. Kelly 2009 reported completer-only CGI-S endscores at 8 weeks. Poyurovsky 2003 reported CGI-S endscores at 6 weeks which were skewed and we entered these into Analysis 1.14 and calculated change scores with estimated SD. Poyurovsky 2007 reported ITT CGI-S change scores at 6 weeks and we could estimate endscores and impute the SD. Schutz 2001 reported completer CGI$\mathrm{S}$ and CGI-I endpoint scores at 6 weeks and the latter were skewed and entered into Analysis 1.14.

Five studies $(n=160)$ provided CGI-S scores and they did not show an overall effect of NRIs (MD $-0.03,95 \% \mathrm{Cl}-0.35$ to 0.28 ; Analysis 1.13). We performed subgroup and sensitivity analysis (see Table 9). There was only one study of atomoxetine which did not show a statistically significant benefit (Kelly 2009); and excluding this to look at reboxetine resulted in a small benefit for the control group which was not statistically significant (MD $0.10,95 \% \mathrm{Cl}-0.23$ to 0.42). Only Hinkelmann 2013 included patients taking clozapine and specifically recruited those with negative symptoms and this showed a benefit of placebo that was not statistically significant. Excluding imputed SDs slightly inflates the benefit of NRIs but remains non-significant (MD $-0.15 ; 95 \% \mathrm{Cl}-0.60$ to 0.29 ) - these were also the studies with skewed endscores (Hinkelmann 2013; Poyurovsky 2003). A fixed-effect model made no difference. All trials were low risk of bias for randomisation. We considered Kelly 2009 to be at high risk for other sources of bias due to apparently changing exclusion criteria after randomisation.

\subsection{Leaving the study early: 1a. Short term (2 to 12 weeks)}

\subsection{1 any reason}

Li 2008 reported 4/115 withdrawals which could not be assigned to a treatment arm and Sacco 2009 did not report withdrawals. Eight trials $(n=401)$ reported little difference (RR $0.94,95 \% \mathrm{Cl} 0.63$ to 1.39; Analysis 1.15). One of these eight trials had no withdrawals so did not contribute to the overall estimate (Shafti 2015).

We performed subgroup and sensitivity analysis (see Table 10). There was little difference when atomoxetine or reboxetine trials were considered separately and the single viloxazine study showed a similar lack of effect (Kurland 1981). No study included only patients on clozapine and only Hinkelmann 2013 included any patients taking clozapine and this study showed a non-significant benefit for placebo. This was one of only two studies (the other being Shafti 2015) recruiting patients specifically with negative symptoms. A fixed-effect model made no difference to the relative risk. No study was at high risk of bias for randomisation and five studies reported their methods in enough detail to be considered low risk; analysing these separately made little difference (Hinkelmann 2013; Kelly 2009; Poyurovsky 2003; Poyurovsky 2007; Schutz 2001). We rated two studies as high risk of other sources of bias (Eli Lilly 2006; Kelly 2009), two studies were high risk for reporting bias (Eli Lilly 2006; Kurland 1981), and one study (Kelly 2009) at high risk of attrition bias; but excluding these made little difference.

\subsection{2 due to adverse effects}

Four studies $(n=178)$ showed a non-significant benefit of placebo (RR 2.08, 95\% Cl 0.70 to 6.21; Analysis 1.15).

We performed subgroup and sensitivity analysis for short-term outcomes (see Table 11). Only Schutz 2001 looked at reboxetine but this showed little difference from the atomoxetine studies. None of the studies included patients taking clozapine and none recruited specifically those with negative symptoms. A fixed-effect model made no difference. No study was at high risk of bias for randomisation but only two studies were low risk and these showed contrasting non-significant benefits for NRI or placebo respectively and no overall effect when combined (RR $1.15,95 \% \mathrm{Cl} 0.15$ to 8.60 ) (Kelly 2009; Schutz 2001). Two studies were at high risk for other sources of bias (Eli Lilly 2006; Kelly 2009); and excluding these magnified the benefit of placebo (RR $4.48,95 \% \mathrm{Cl} 0.56$ to 35.52 ). Eli Lilly 2006 also showed attrition and reporting bias and excluding this slightly attenuated the benefit of placebo which remained nonsignificant.

\subsection{3 due to psychiatric symptoms}

Eli Lilly 2006 and Kelly 2009 reported withdrawal due to psychiatric symptoms at 8 weeks. Poyurovsky 2007 reported withdrawal due to lack of efficacy at 6 weeks.

Combining the three studies $(n=183)$ reporting withdrawal due to psychiatric symptoms (variously described) showed no evident difference between NRIs and placebo (RR 0.97, 95\% $\mathrm{Cl} 0.44$ to 2.13; Analysis 1.15). 
There were only three trials so we did not perform a full subgroup and sensitivity analysis but fixed-effect analysis made no difference (RR $1.01,95 \% \mathrm{Cl} 0.47$ to 2.20 ).

\subsection{Leaving the study early: 1b. Medium term (13 to 26 weeks)}

\subsection{1 any reason}

Zhao 2013 reported 3/110 withdrawals which could not be assigned to a treatment arm and Ganguli 2008 did not report withdrawals.

Three trials $(n=169)$ reported medium-term (13 to 26 weeks) outcomes (RR $0.92,95 \% \mathrm{Cl} 0.52$ to 1.62 ; Analysis 1.16) with little difference (Ball 2011; Usall 2014; Yu 2012).There were only three trials so a full subgroup and sensitivity analysis was not performed but a fixed-effect model made little difference (RR 0.88, 95\% Cl 0.50 to 1.55$)$.

\subsection{2 due to adverse effects}

The atomoxetine trial by Ball 2011 reported outcomes at 24 weeks (RR $1.47,95 \% \mathrm{Cl} 0.31$ to 6.95 ; Analysis 1.16 ) and showed a nonsignificant trend to favour controls.

\subsection{3 due to psychiatric symptoms}

Usall 2014 reported withdrawal due to acute exacerbation at 24 weeks.

This showed a benefit to placebo that was not statistically significant (RR 4.44, 95\% Cl 0.22 to 88.04; Analysis 1.16).

\subsection{Adverse effects: 1. General - short term (binary, 2 to 12 weeks)}

\subsection{1 any}

The Eli Lilly 2006 study of atomoxetine reported treatmentemergent adverse events as percentages which we could use to determine the number of events in each group at 8 weeks and this showed little difference between arms (RR 1.07, 95\% $\mathrm{Cl} 0.80$ to 1.43; Analysis 1.17). The Kurland 1981 trial of viloxazine reported that there were no adverse events at 4 weeks. Shafti 2015 reported sideeffects in the reboxetine arm at 12 weeks and, assuming that there were none in the placebo arm, this implies a very large risk of sideeffects associated with reboxetine (RR $19.00,95 \% \mathrm{Cl} 1.17$ to 309.77 ). When combined this indicates a large non-significant benefit for placebo (RR 3.49, 95\% Cl 0.14 to 90.29 ; Analysis 1.17) with large heterogeneity $\left(I^{2}=82 \%\right)$.

\subsection{2 serious}

The Eli Lilly 2006 study of atomoxetine reported serious adverse events as percentages which we used to determine the number of events in each group at 8 weeks. This showed an increased rate of serious adverse events with atomoxetine that was not statistically significant (RR $2.95,95 \% \mathrm{Cl} 0.32$ to 27.58 ). Hinkelmann 2013 reported that there were no serious adverse events at 4 weeks in either reboxetine or placebo arms. Li 2008 reported one seizure in the reboxetine arm of the study. Overall these showed a nonsignificant benefit of placebo (RR 2.95, 95\% Cl 0.47 to 18.36 ; Analysis 1.17). No deaths were reported in any study.

\subsection{Adverse effects: 2a.i. Specific - short term (binary, 2 to 12 weeks)}

Kelly 2009 reported rates of nausea, insomnia, dry mouth, constipation, sedation, malaise (weakness, fatigue), dizziness, anorexia, abdominal pain, vomiting, diarrhoea, sore throat, weight loss, tremor, akathisia, restlessness, and stiffness over 8 weeks in completers and we calculated ITT figures. Li 2008 reported rates of nausea, insomnia, dry mouth, constipation, dizziness, and tachycardia over 12 weeks in completers and we could not calculate ITT figures as withdrawals were not reported by study arm. Poyurovsky 2003 reported rates of daytime somnolence, akathisia, and use of anticholinergic medication over 6 weeks in completers and we calculated ITT figures. They also reported no gastrointestinal side-effects (0/13 in both arms). Schutz 2001 reported rates of nausea, dry mouth, fatigue, dizziness, impotence, rash, akathisia, parkinsonism, acute dystonia, and hypersalivation over 6 weeks in completers and we calculated ITT figures. Eli Lilly 2006 reported percentages which we used to calculate rates of headache at 8 weeks. Poyurovsky 2003 reported that no anticholinergic medication was used in either the reboxetine or placebo arms over 6 weeks. Poyurovsky 2007 and Shafti 2015 reported rates of anticholinergic medication at 6 or 12 weeks respectively and we assumed these to be ITT.

\subsection{1 anticholinergic - constipation}

One study showed a benefit for atomoxetine which was not significant (Kelly 2009); and one reboxetine study showed no effect (Li 2008). Overall there was a non-significant benefit for NRIS (RR $0.74,95 \% \mathrm{Cl} 0.24$ to 2.29 ; Analysis 1.18 ).

\subsection{2 anticholinergic - dry mouth}

Combining three studies $(n=176)$ showed an adverse effect of NRIs (RR 3.46, 95\% Cl 1.40 to 8.53; Analysis 1.18).

As there were only three trials a full subgroup and sensitivity analysis was not performed but fixed-effect analysis made little difference (RR $3.55,95 \% \mathrm{Cl} 1.45$ to 8.70 ), nor did using completer rather than ITT data (RR 3.35, 95\% 1.35 to $8.30 ; \mathrm{N}=163$ ).

\subsection{3 anticholinergic - impotence}

Schutz 2001 found no difference between reboxetine and placebo (Analysis 1.18).

\subsection{4 cardiovascular - tachycardia}

For reboxetine, Li 2008 reported rates of tachycardia at 12 weeks (RR 3.33, 95\% Cl 0.97 to 11.48 ) showing a benefit of placebo that was not statistically significant (Analysis 1.18).

\subsection{5 central nervous system - anorexia}

Kelly 2009 showed no difference between atomoxetine and placebo (Analysis 1.18).

\subsection{6 central nervous system - dizziness}

Combining these three studies $(n=176)$ did not suggest an effect of NRIs (RR 1.52, 95\% Cl 0.54 to 4.31; Analysis 1.18).

There were only three trials so a full subgroup and sensitivity analysis was not performed but fixed-effect analysis made little difference (RR $1.55,95 \% \mathrm{Cl} 0.55$ to 4.33 ), nor did using completer rather than ITT data (RR $1.60,95 \% \mathrm{Cl} 0.57$ to 4.52 ).

\subsection{7 central nervous system - fatigue}

One atomoxetine (Kelly 2009) and one reboxetine (Schutz 2001) study gave no suggestion of an overall effect of NRIs (RR 1.00, 95\% $\mathrm{Cl} 0.12$ to 8.60 ; Analysis 1.18). 


\subsection{8 central nervous system - headache}

Eli Lilly 2006 found no difference between atomoxetine and placebo (Analysis 1.18).

\subsection{9 central nervous system - insomnia}

One atomoxetine (Kelly 2009) and one reboxetine (Li 2008) study showed a non-significant benefit for placebo (Analysis 1.18).

\subsubsection{0 central nervous system - sedation}

Combining one atomoxetine (Kelly 2009) and one reboxetine (Poyurovsky 2003) study produced a non-significant benefit of NRIs (RR 0.74, 95\% Cl 0.28 to 1.94; Analysis 1.18).

\subsubsection{1 extrapyramidal - acute dystonia}

Schutz 2001 reported no difference between arms (RR 1.00, 95\% Cl 0.07 to 14.55 ; Analysis 1.18).

\subsubsection{2 extrapyramidal - akathisia}

Kelly 2009 reported rates of akathisia with a non-significant benefit for atomoxetine and also rates of restlessness which were the same in each arm. Poyurovsky 2003 reported no akathisia in either the reboxetine or placebo arm while the rates in Schutz 2001 were the same in each arm. Overall there was a marginal and non-significant benefit for NRIs (RR 0.87, 95\% Cl 0.25 to 3.09; Analysis 1.18).

\subsubsection{3 extrapyramidal - hypersalivation}

Schutz 2001 reported a non-significant benefit for placebo on hypersalivation (RR 0.33, 95\% Cl 0.01 to 7.58 ; Analysis 1.18).

\subsubsection{4 extrapyramidal - parkinsonism}

Schutz 2001 showed a non-significant benefit for placebo (RR 1.80, $95 \% \mathrm{Cl} 0.79$ to 4.11 ; Analysis 1.18).

\subsubsection{5 extrapyramidal - tremor}

Kelly 2009 found no difference between the two arms (RR 1.00, 95\% $\mathrm{Cl} 0.24$ to 4.23 ; $\mathrm{N}=32$; Analysis 1.18 )

\subsubsection{6 extrapyramidal - stiffness}

Kelly 2009 showed a non-significant benefit for atomoxetine (RR 5.00, $95 \% \mathrm{Cl} 0.66$ to 38.15; Analysis 1.18).

\subsubsection{7 extrapyramidal - use of antiparkinson medication}

Poyurovsky 2003 reported no use in either the reboxetine or placebo arms while combining Poyurovsky 2007 and Shafti 2015 showed little benefit of reboxetine (RR $0.80,95 \% \mathrm{Cl} 0.44$ to 1.45 ; Analysis 1.18).

\subsubsection{8 gastrointestinal - abdominal pain}

Kelly 2009 showed no difference between atomoxetine and placebo (Analysis 1.18).

\subsubsection{9 gastrointestinal - nausea}

Three studies ( $\mathrm{n}=176$ ) showed a non-significant benefit for NRIs (RR 0.49, 95\% Cl 0.10 to 2.41; Analysis 1.18).

As there were only three trials we did not perform a full subgroup and sensitivity analysis but fixed-effect analysis made little difference (RR $0.63 ; 95 \% \mathrm{Cl} 0.28$ to 1.43 ), nor did using completer rather than ITT data (RR $0.60 ; 95 \% \mathrm{Cl} 0.15$ to $2.41 ; \mathrm{N}=$ 163).

\subsubsection{0 gastrointestinal - vomiting}

Kelly 2009 showed no difference between atomoxetine and placebo (Analysis 1.18).

\subsubsection{1 gastrointestinal - diarrhoea}

Kelly 2009 found no difference between atomoxetine and placebo (Analysis 1.18).

\subsubsection{2 immune system - rash}

Schutz 2001 showed no difference between reboxetine and placebo (Analysis 1.18).

\subsubsection{3 immune system - sore throat}

Kelly 2009 found no difference between atomoxetine and placebo (Analysis 1.18).

\subsubsection{4 metabolic - weight loss}

Kelly 2009 showed no difference between atomoxetine and placebo (Analysis 1.18).

\subsubsection{5 metabolic - significant weight gain}

Two reboxetine studies reported rates of weight gain, both using the criterion of $7 \%$ or more increase in weight. Poyurovsky 2003 reported weight gain for completers at 6 weeks and we calculated ITT rates from this while Poyurovsky 2007 reported ITT weight gain at 6 weeks. Both studies showed a significant benefit for reboxetine which was demonstrated when they were combined (RR $0.38,95 \% \mathrm{Cl} 0.20$ to 0.73 ; Analysis 1.18 ). This benefit persists utilising completer only rather than ITT data for Poyurovsky 2003 (RR 0.37, 95\% Cl 0.19 to $0.75 ; \mathrm{N}=79$ ).

\subsection{Adverse effects: 2 a.ii. Specific - extrapyramidal - average change score (continuous, various scales, high = worse) - short term (2 to 12 weeks)}

Kelly 2009 reported SAS and AIMS endscores for atomoxetine completers at 8 weeks which were skewed and entered into Analysis 1.20 and we calculated change scores with SD imputed. Poyurovsky 2007 reported SAS and BAS ITT change scores at 6 weeks for reboxetine: we calculated endscores with estimated SDs, and the BAS endscore was skewed. Schutz 2001 reported SAS endscores for reboxetine completers at 6 weeks which were skewed and entered into Analysis 1.20 but it was not possible to calculate change scores as baseline values were not available.

\subsubsection{AIMS}

AIMS change scores from Kelly 2009 showed no benefit of atomoxetine over placebo (MD 0.30, 95\% Cl -2.34 to 2.94; Analysis 1.19).

\subsubsection{BAS}

BAS change scores from Poyurovsky 2007 showed no benefit of reboxetine over placebo (MD $-0.18,95 \% \mathrm{Cl}-0.65$ to 0.29 ; Analysis 1.19). 


\subsubsection{SAS}

SAS change scores from Poyurovsky 2007 and Kelly 2009 showed minimal benefit of NRIs (MD $-0.27,95 \% \mathrm{Cl}-1.79$ to 1.25 ; Analysis 1.19).

\subsection{Adverse effects: 2 b.i. Specific - medium term (binary, 13 to 26 weeks)}

The reboxetine study of Zhao 2013 reported rates of nausea, insomnia, dry mouth, constipation, dizziness, tachycardia, and sweating over 24 weeks including all completers with no withdrawals. Ball 2011 reported rates of tremor and QT prolongation over 24 weeks in completers and we calculated ITT figures.

\subsection{1 anticholinergic - constipation}

There was a statistically significant benefit for placebo (RR 4.58, 95\% Cl 1.04 to 20.23; Analysis 1.21).

\subsection{2 anticholinergic - dry mouth}

This showed a small but non-significant benefit for placebo (RR 1.53, 95\% Cl 0.27 to 8.78; Analysis 1.21 ).

\subsection{3 anticholinergic - sweating}

Zhao 2013 showed no difference between reboxetine and placebo (Analysis 1.21).

\subsection{4 cardiovascular - QT prolongation}

Ball 2011 showed no difference between atomoxetine and placebo (Analysis 1.21).

\subsection{5 cardiovascular - tachycardia}

There was a benefit of placebo that was not statistically significant (RR 1.78, 95\% Cl 0.55 to 5.74; Analysis 1.21).

\subsection{6 central nervous system - dizziness}

This did not suggest an effect of reboxetine (RR 1.36, 95\% Cl 0.32 to 5.78; Analysis 1.21).

\subsection{7 central nervous system - insomnia}

We found a non-significant benefit for placebo (Analysis 1.21).

\subsection{8 extrapyramidal - tremor}

Ball 2011 showed a significant benefit of atomoxetine (RR 0.42; 95\% $\mathrm{Cl} 0.20$ to 0.89; Analysis 1.21).

\subsection{9 gastrointestinal - nausea}

We calculated a large, but not statistically significant, benefit for placebo (RR 5.09, 95\% Cl 0.62 to 42.16; Analysis 1.21).

\subsection{Adverse effects: 2a.iv. Specific - metabolic - average weight gain (continuous, increase in $\mathrm{kg}$ )}

Ball 2011 did not report weight gain at 24 weeks directly but we could estimate mean change from the figure and SD from the mixed model. Poyurovsky 2003 reported weight gain at 6 weeks. Poyurovsky 2007 reported ITT weight gain at 6 weeks. Zhao 2013 reported endpoint weight and we calculated weight gain at 8 weeks and 24 weeks with estimated SD. Endpoint weights were available or could be estimated for all studies with no evidence of skew.

\subsection{1 short term ( 2 to 12 weeks)}

Three studies $(n=186)$ showed a significant benefit of reboxetine (MD -2.17, 95\% Cl -3.19 to -1.15; Analysis 1.22).

There were only three trials so we did not perform a full subgroup and sensitivity analysis but a fixed-effect model made no difference (MD $-2.17,95 \% \mathrm{Cl}-3.19$ to -1.15 ).

\subsection{2 medium term (13 to 26 weeks)}

We found a non-significant benefit of NRIs (MD $-3.12,95 \% \mathrm{Cl}-10.67$ to 4.42; Analysis 1.22) with very high heterogeneity $\left(I^{2}=98 \%\right)$ from two studies. Ball 2011 found little effect of atomoxetine while Zhao 2013 showed a large benefit with reboxetine.

\subsection{Social or general functioning: Average social functioning score (various subscales) - short-term (2 to 12 weeks)}

The atomoxetine study of Friedman 2008 reported LOCF and completer change scores for the six SLOF subscales at 8 weeks. We analysed the change scores for completers but were able to calculate LOCF endscores with estimated SD and these were not skewed. We inverted these endscores were inverted by subtracting from zero in order to display them in the same direction as the SSPI scale. With the SLOF, inverted high scores are now worse. The reboxetine study by Li 2008 reported endscores for the ten SSPI subscales at 12 weeks. Three of these subscales were skewed and we calculated change scores with estimated SD.

1.23.1 activity - activities (inverted SLOF, high = worse)

Friedman 2008 showed no benefit of atomoxetine (MD 0.00, 95\% CI -3.30 to 3.30 ; Analysis 1.23 ).

\subsection{2 activity - hospital activity (SSPI, high = worse)}

Li 2008 found no benefit of reboxetine (MD $-0.03,95 \% \mathrm{Cl}-0.17$ to 0.11 ; Analysis 1.23).

\subsection{3 activity - disease indoor activity (SSPI, high = worse)}

This was analysed as a change score due to the subscale being skewed and Li 2008 showed no benefit of reboxetine (MD - $0.14,95 \%$ $\mathrm{Cl}-0.28$ to -0.00 ; Analysis 1.23 ).

\subsection{4 activity - physical functioning (inverted SLOF, high = worse)}

Friedman 2008 showed no benefit of atomoxetine (MD -0.30 , 95\% $\mathrm{Cl}-1.12$ to 0.52 ; Analysis 1.23).

\subsection{5 personal care - personal care skills (inverted SLOF, high = worse)}

Friedman 2008 showed no benefit of atomoxetine (MD -0.20, 95\% $\mathrm{Cl}-1.43$ to 1.03 ; Analysis 1.23).

\subsection{6 personal care - self-care (SSPI, high = worse)}

We analysed this as a change score due to the subscale being skewed and Li 2008 showed no benefit of reboxetine (MD -0.03, 95\% $\mathrm{Cl}-0.22$ to $0.16 ; \mathrm{N}=114$; Analysis 1.23 ).

\subsection{7 personal care - social acceptability (inverted SLOF, high = worse)}

Friedman 2008 showed no benefit of atomoxetine (MD 0.10, 95\% Cl -0.72 to 0.92 ; Analysis 1.23 ). 
1.23.8 relationships - family role (SSPI, high = worse)

Li 2008 showed no benefit of reboxetine (MD $-0.09,95 \% \mathrm{Cl}-0.23$ to 0.05; Analysis 1.23).

1.23.9 relationships - interest and concern in the external environment (SSPI, high $=$ worse)

Li 2008 found a small benefit of reboxetine (MD $-0.19,95 \% \mathrm{Cl}-0.37$ to $-0.01 ; \mathrm{N}=114$; Analysis 1.23 )

1.23.10 relationships - interpersonal relationships (inverted SLOF, high $=$ worse)

Friedman 2008 showed no benefit of atomoxetine (MD -1.20, 95\% $\mathrm{Cl}-5.41$ to 3.01 ; Analysis 1.23).

1.23.11 relationships - relationships and caring for others (SSPI, high = worse)

Li 2008 showed no benefit of reboxetine (MD $-0.09,95 \% \mathrm{Cl}-0.24$ to 0.06; Analysis 1.23).

\subsubsection{2 relationships - sexual role (SSPI, high $=$ worse)}

Li 2008 showed no benefit of reboxetine (MD $-0.03,95 \% \mathrm{Cl}-0.21$ to 0.15 ; Analysis 1.23).

\subsubsection{3 relationships - social withdrawal (SSPI, high = worse)}

We analysed this as a change score due to the subscale being skewed and Li 2008 showed a benefit of reboxetine (MD - $0.32,95 \%$ $\mathrm{Cl}-0.49$ to -0.15 ; Analysis 1.23).

\subsubsection{4 work - professional skills (SSPI, high = worse)}

Li 2008 showed a small benefit of reboxetine (MD $-0.19,95 \% \mathrm{Cl}$ -0.34 to -0.04 ; Analysis 1.23 )

1.23.15 work - responsibility and planning (SSPI, high = worse)

Li 2008 showed no benefit of reboxetine (MD $-0.03,95 \% \mathrm{Cl}-0.21$ to 0.15 ; Analysis 1.23).

\subsubsection{6 work - work skills (inverted SLOF, high = worse)}

Friedman 2008 showed a benefit of atomoxetine (MD $-3.00,95 \% \mathrm{Cl}$ -5.48 to -0.52 ; Analysis 1.23$)$.

\section{Missing outcomes}

There were no reported results from any study for two of the primary outcomes: significant response or improvement in a clinical scale of cognitive functioning or in quality of life. While many studies measured the PANSS positive subscale, the SAPS, and the HRSD, none defined or reported response or relapse rates. No studies reported scores for any other symptom scales not mentioned above. No studies reported rates of response or improvement for composite cognitive scores or in any individual cognitive domain (e.g. working memory). No studies reported occupational status, economic, or service utilisation outcomes (e.g. admission rates). No study reported 'significant' extrapyramidal side-effects or movement disorder but they did report rates of specific extrapyramidal symptoms and no studies reported cumulative dosage of antiparkinson medication. No studies reported any measures of satisfaction with treatment, the general impression of carers beyond the CGI, or improvement in social functioning.

\section{Sensitivity analysis}

There was not sufficient data to combine studies in a meta-analysis so we did not perform a subgroup and sensitivity analysis on the primary outcome measures.

\section{Comparison 2. NRI versus citalopram}

Only two trials provided data to compare NRIs against an active control - Hinkelmann 2013 and Usall 2014 - and both compared reboxetine to citalopram, but at 4 weeks and 24 weeks respectively, so they could not be combined in a meta-analysis.

\subsection{Mental state: 1a. General: Average general/overall symptoms score (PANSS general, high = worse) - short term (2 to 12 weeks)}

Hinkelmann 2013 reported LOCF PANSS general endpoint scores at 4 weeks which were skewed and we entered these into Analysis 2.5 and calculated LOCF PANSS general change scores with an estimated SD.

We found a a non-significant benefit of citalopram over reboxetine (MD 2.90, 95\% Cl-2.85 to 8.65; Analysis 2.1).

\subsection{Mental state: 1 b. General: Average general/overall symptoms score (various scales, high $=$ worse) - medium term (13 to 26 weeks)}

Usall 2014 reported PANSS total and general endpoint scores at 24 weeks which were skewed and we entered into Analysis 2.6. We calculated change scores with an estimated SD.

\subsubsection{PANSS total}

Usall 2014 found a non-significant trend for a benefit of citalopram over reboxetine (MD 1.62, $95 \% \mathrm{Cl}-6.89$ to 10.13 ; Analysis 2.2).

\subsubsection{PANSS general}

Usall 2014 found little difference (MD $0.62,95 \% \mathrm{Cl}-4.00$ to 5.24 ; Analysis 2.2) at 24 weeks.

\subsection{Mental state: $2 a$. Specific: Average symptoms score (various scales, high $=$ worse) - short term (2 to 12 weeks)}

Hinkelmann 2013 reported LOCF PANSS negative, HRSD, and PANSS positive endpoint scores at 4 weeks but these were skewed and we entered them into Analysis 2.5. We calculated change scores and imputed the SDs.

\subsection{1 mood (HRSD)}

This showed a fairly large but non-significant benefit of citalopram over reboxetine (MD 3.00, 95\% Cl-1.24 to 7.24; Analysis 2.3).

\subsection{2 negative (PANSS negative)}

Hinkelmann 2013 found a non-significant benefit of citalopram over reboxetine (MD 1.50, 95\% Cl -4.34 to 7.34; Analysis 2.3).

\subsection{3 positive (PANSS positive)}

We found a non-significant benefit of citalopram over reboxetine (MD 1.70, 95\% Cl -1.52 to 4.92; Analysis 2.3). 
2.4 Mental state: $2 b$. Specific: Average symptoms score (various scales, high $=$ worse) - medium term (13 to 26 weeks)

Usall 2014 reported PANSS negative and SANS endpoint scores at 24 weeks which were skewed and entered into Analysis 2.6 and we calculated change scores with imputed SDs.

\subsection{1 negative (PANSS negative)}

Usall 2014 found little difference (MD $0.26,95 \% \mathrm{Cl}-3.66$ to 4.18; Analysis 2.4).

\subsection{2 negative (SANS)}

There was minimal difference between reboxetine and citalopram (MD $-0.27,95 \% \mathrm{Cl}-12.69$ to 12.15 ; Analysis 2.4).

\subsection{3 positive (PANSS positive)}

Usall 2014 found little difference (MD $0.74,95 \% \mathrm{Cl}-1.86$ to 3.34 Analysis 2.4).

\subsection{Clinical global response: Average clinical global status score (CGI-S, high $=$ worse) - short term (2 to 12 weeks)}

Hinkelmann 2013 reported CGI-S endscores at 4 weeks which were not skewed and showed no difference between reboxetine and citalopram (MD 0.00, $95 \% \mathrm{Cl}-0.89$ to 0.89 ; Analysis 2.7 ).

\subsection{Leaving the study early: 1a. Short term (2 to 12 weeks)}

\subsection{1 any reason}

In the short-term Hinkelmann 2013 found a non-significant benefit of reboxetine over citalopram ( $\mathrm{RR} 0.63,95 \% \mathrm{Cl} 0.17$ to 2.41 ; Analysis 2.8) at 4 weeks. Hinkelmann 2013 reported that there were no serious adverse events at 4 weeks in either reboxetine or citalopram arms.

\subsection{Leaving the study early: $1 \mathrm{~b}$. Medium term (13 to 26 weeks)}

\subsection{1 any reason}

At 24 weeks Usall 2014 found little difference (RR 1.13, 95\% Cl 0.48 to 2.67; Analysis 2.9). No deaths were reported.

\subsection{2 due to psychiatric symptoms}

Usall 2014 reported withdrawal due to acute exacerbation at 24 weeks which showed a non-significant benefit for citalopram over reboxetine (RR 1.77, 95\% Cl 0.17 to 18.26; Analysis 2.9).

\section{I SCUSSION}

\section{Summary of main results}

Sixteen studies (39 articles, 919 participants) met the inclusion criteria for this review, although only 14 studies contributed useable data for the meta-analysis. Below we discuss the main findings outlined in the Summary of findings for the main comparison plus selected additional adverse effects.

\section{Mental state}

\subsection{Significant response or improvement in negative symptoms}

Only one study reported this outcome in a useable form. Shafti 2015 found a much greater response rate $(20 \%$ reduction in the SANS) with reboxetine at 12 weeks compared to placebo (Analysis 1.1) and is included in the Summary of findings for the main comparison. Due to the limited evidence available we have rated this finding as 'very low quality' and it should be interpreted with great caution. We therefore considered that it would be misleading to focus on this outcome in isolation when continuous outcomes offer complementary and comparable information to dichotomous outcomes. Therefore we briefly discuss the results from average negative symptom scores below.

\subsubsection{Average negative symptom score}

Three studies $(N=129)$ reported SANS continuous scores in the short-term (2 to 12 weeks) and combining these we found no benefit of reboxetine on negative symptoms compared to placebo (Poyurovsky 2003; Poyurovsky 2007; Shafti 2015). However, there was substantial heterogeneity and the effect was not robust with a fixed-effect model showing a statistically significant benefit of reboxetine. Kelly $2009(\mathrm{~N}=23)$ reported a modified version of the SANS in the short term (2 to 12 weeks) which did not show a significant benefit.

Six studies $(N=359)$ reported PANSS negative subscale continuous scores in the short term ( 2 to 12 weeks) and we found no benefit of NRIs over placebo (Friedman 2008; Hinkelmann 2013; Li 2008; Schutz 2001; Yu 2012; Zhao 2013). There was again significant heterogeneity and the effect was not robust as, for example, a fixedeffect model showed a statistically significant benefit of NRIs.

In the medium term (13 to 26 weeks) one study showed no benefit of reboxetine on the SANS (Usall 2014; $N=47$ ) while three studies $(\mathrm{N}=219)$ reporting the PANSS negative subscale suggested a large benefit of reboxetine over placebo (Usall 2014; Yu 2012; Zhao 2013). The medium term PANSS negative subscale result is not included in the Summary of findings for the main comparison and we consider it to be 'low quality' by GRADE criteria due to concerns about small sample size and the risk of bias in some of the trials and so it should be interpreted with caution.

The more equivocal findings in the short term could represent a correlation between duration of treatment and effect size and this is borne out by the larger benefit of reboxetine in the medium term compared to the short term seen in the two studies contributing to both analyses (Yu 2012; Zhao 2013).

\subsection{Significant response or improvement in positive symptoms}

No trials reported this outcome so we used average positive symptom score as a surrogate and downgraded the quality of evidence due to indirectness as per GRADE criteria.

\subsubsection{Average positive symptoms score}

Three studies $(N=129)$ reported SAPS continuous scores in the short term (2 to 12 weeks) and we found no effect of reboxetine (Poyurovsky 2003; Poyurovsky 2007; Shafti 2015). One study (Kelly 2009; $N=23$ ) reported short-term BPRS positive subscale continuous scores and we found no difference between atomoxetine and placebo.

Five studies $(N=294)$ reported PANSS positive subscale continuous scores in the short term ( 2 to 12 weeks) with no effect of NRIs over placebo (Friedman 2008; Hinkelmann 2013; Li 2008; Schutz 2001; Zhao 2013). We included this in the Summary of findings for the main comparison and rated it as 'moderate quality' due to the small sample size. As a surrogate for the dichotomous outcome we further downgraded to 'low quality' due to the indirectness of 
the measure and it should be interpreted with caution. The lack of effect of NRIs on positive symptoms in the short term (2 to 12 weeks) was quite robust although the confidence intervals did not completely exclude clinically significant effects.

Two further studies also reported medium-term (13 to 26 weeks) outcomes without effect of reboxetine (Usall 2014; Zhao 2013; $N=$ 154).

\section{Cognitive functioning}

\subsection{Significant response or improvement in clinical scale of cognitive functioning}

No trial reported this outcome.

\subsection{Average composite cognitive functioning score}

Four studies $(N=180)$ reported composite SMD effect sizes in the short term ( 2 to 12 weeks) with no benefit for NRIs over placebo (Eli Lilly 2006; Friedman 2008; Kelly 2009; Poyurovsky 2007). and when we combined them there was a fairly robust lack of benefit with confidence intervals excluding all but a small benefit of NRIs. We included this result in the Summary of findings for the main comparison and rated it as 'low quality' due to the small sample size, width of the confidence intervals, and risk of bias in some studies.

One study reported medium-term (13 to 26 weeks) outcomes for atomoxetine without a significant benefit (Ball 2011; $\mathrm{N}=26$ ).

\section{Quality of life}

\subsection{Significant response or improvement in quality of life}

No trial reported this outcome so we used average quality of life score as a surrogate and downgraded the quality of evidence due to indirectness as per GRADE criteria.

\subsubsection{Average quality of life score}

One trial reported GQOLI-74 total and subscale continuous scores in the short-term ( 2 to 12 weeks) and we found a benefit of reboxetine on the total score and on two out of four subscales (psychological and social wellbeing) compared to placebo ( $\mathrm{Li} 2008$; $\mathrm{N}=114$ ). We included this result in the Summary of findings for the main comparison and rated it as 'very low quality' due to concerns about the risk of bias in this trial and evidence of publication bias and it should be interpreted with great caution.

\section{Clinical global response}

\subsection{Significant response or improvement in clinical global status}

One study reported non-specific improvement rates which showed no difference between placebo and viloxazine (Kurland 1981; N $=28$ ). We included this result in the Summary of findings for the main comparison and rated it as 'very low quality' due to concerns about the quality of the trial and the risk of bias as well as evidence of publication bias and it should be interpreted with great caution. We therefore considered that it would be misleading to focus on this outcome in isolation when continuous outcomes offer complementary and comparable information to dichotomous outcomes. Therefore we briefly discuss the results from average clinical global status score below.

\subsubsection{Average clinical global status score}

Five studies $(n=160)$ reported CGI-S continuous scores in the short term ( 2 to 12 weeks) and we found no benefit for NRIs over placebo (Hinkelmann 2013; Kelly 2009; Poyurovsky 2003; Poyurovsky 2007; Schutz 2001). These findings were robust although the confidence intervals could not exclude a clinically significant benefit. This result was not included in the Summary of findings for the main comparison and we have rated it as 'moderate quality' by GRADE criteria due to the small sample size.

\section{Leaving the study early}

\subsection{All cause withdrawals}

Eight trials ( $n=401)$ reported short-term ( 2 to 12 weeks) outcomes and we found no difference in the rates of withdrawals between NRIs and placebo (Eli Lilly 2006; Friedman 2008; Hinkelmann 2013; Kelly 2009; Poyurovsky 2003; Poyurovsky 2007; Schutz 2001; Shafti 2015). Three trials $(n=169)$ reported medium-term ( 13 to 26 weeks) outcomes with similar results (Ball 2011; Usall 2014; Yu 2012). This lack of effect was robust but the confidence intervals did not rule out clinically significant benefits or harms; and withdrawals were poorly reported. We did not include this result in the Summary of findings for the main comparison and we have rated it as 'moderate quality' using GRADE criteria due to concerns about the small sample size and wide confidence intervals.

\subsection{Withdrawal due to adverse events}

Four studies $(n=178)$ reported results in the short term ( 2 to 12 weeks) and we found no difference in withdrawals due to adverse events between NRIs and placebo (Eli Lilly 2006; Friedman 2008; Kelly 2009; Schutz 2001). One medium-term (24 weeks) study (N = 33) of atomoxetine also showed no difference (Ball 2011). The confidence intervals did not rule out clinically significant benefits or harms, and withdrawals were poorly reported. We did not include this in the Summary of findings for the main comparison and we have rated it as 'low quality' using GRADE criteria due to concerns about small sample size, wide confidence intervals, and likely publication bias, and it should be interpreted with caution.

\section{Specific adverse effects}

\subsection{Incidence of nausea}

We were unable to include the data from one study (Hinkelmann 2013).

Three studies $(n=176)$ reported rates of nausea in the short term ( 2 to 12 weeks) but we found no evidence of an increased rate with NRIs over placebo (Kelly 2009; Li 2008; Schutz 2001). While this was fairly robust the confidence intervals do not rule out clinically significant benefits or harms and, overall, adverse events were poorly reported and the number of events was low. This result is included in the Summary of findings for the main comparison and we have rated it as 'low quality' due to concerns about publication bias, small sample size, and wide confidence intervals, and it should be interpreted with caution.

One study reported rates in the medium term (13 to 26 weeks) without a significantly increased rate with reboxetine (Zhao 2013; $\mathrm{N}=107$ ). 


\subsection{Average weight gain}

Three studies ( $\mathrm{n}=186)$ reported weight gain in the short term (2 to 12 weeks) and we found that patients taking reboxetine gained $2 \mathrm{~kg}$ less on average than patients taking placebo (Poyurovsky 2003; Poyurovsky 2007; Zhao 2013). This result was not included in the Summary of findings for the main comparison and we have rated it as 'low quality' using GRADE criteria due to concerns about publication bias and small sample size, and it should be interpreted with caution.

Two studies reported medium-term (13 to 26 weeks) results, one finding little benefit of atomoxetine (Ball 2011; $N=26$ ), the other a large benefit of reboxetine (Zhao 2013; N = 107).

\subsection{Serious adverse events}

Three studies $(N=271)$ reported rates of serious adverse events in the short term ( 2 to 12 weeks) and we did not find evidence of an increased rate with NRIs compared to placebo (Eli Lilly 2006; Hinkelmann 2013; Li 2008). The confidence intervals did not rule out clinically significant harms and overall adverse events were poorly reported. We did not include this in the Summary of findings for the main comparison and we have rated it as 'very low quality' using GRADE criteria due to concerns about the small sample size with few events and very wide confidence intervals and also likely publication bias and it should be interpreted with great caution.

\subsection{Other specific adverse effects}

We analysed other specific adverse effects, including insomnia, dry mouth, constipation, sedation, fatigue, dizziness, tachycardia, tremor, and akathisia. Single trials reported rates of QTprolongation, headache, vomiting, diarrhoea, anorexia, weight loss, abdominal pain, sore throat, impotence, rash, sweating, parkinsonism, acute dystonia, and hypersalivation. Only for dry mouth did we find rates significantly higher for NRIs over placebo in the short term ( 2 to 12 weeks). In the medium term (13 to 26 weeks) single studies suggested constipation rates were greater with reboxetine while rates of tremor were lower with atomoxetine. These results were not included in the Summary of findings for the main comparison and in general we would rate them as 'very low quality' using GRADE criteria due to concerns about small sample sizes, few events, very wide confidence intervals, and likely publication bias and they should be interpreted with great caution. The elevated rate of dry mouth with NRIs in the short term (2 to 12 weeks) from three studies (Kelly 2009; Li 2008; Schutz 2001; N $=172$ ) was rated as 'low quality' due to concerns about the small sample size and likely publication bias and should be interpreted with caution.

\section{Overall completeness and applicability of evidence}

\section{Completeness}

\subsection{Outcomes}

The majority of reported outcomes were mental state scales (e.g. the PANSS), clinical global response (CGI-S), or cognitive tests. However, even for these outcomes there was evidence of considerable under-reporting and possible publication bias. Few studies attempted to utilise their continuous outcome measures to define response rates and thus there were few dichotomous outcome measures reported. Dichotomous outcomes, such as the proportion of patients with a $50 \%$ reduction in scores on mental state scales, offer complementary information to the mean scores on symptom scales by emphasising the number of patients with clinically significant improvements rather than differences in mean symptom scores averaged across all patients. More patient-centred outcomes (that likely are more relevant for long-term prognosis) such as quality of life, social functioning, occupational status, or carer or patient satisfaction, were rarely reported if at all. Withdrawal rates were around $20 \%$ overall and did not differ between NRI and placebo arms, suggesting that the validity of outcomes was not compromised.

The limited number of small studies included makes detection of rare but serious adverse events highly unlikely, including those of specific interest such as suicide or acute liver failure. For instance, it is very difficult to interpret the finding of one patient developing seizures in the NRI arm of a single study as we found in this review. We did not detect an increased rate of even the most commonly reported side-effect with NRIs (nausea) and given the low event rate it is likely that for most side-effects we did not have the sensitivity to detect an increased rate with NRIs. As is unfortunately often the case in clinical trial reporting, while side-effects were measured by most studies they were infrequently reported in a useable form. Therefore, although NRIs seemed generally relatively well tolerated, there was certainly evidence consistent with NRIs being associated with an increased rate of side-effects.

It is increasingly recognised that the economic costs of interventions are important to guide recommendations for treatment. However, no studies reported on economic outcomes or possible proxies for this, such as hospitalisation.

\subsection{Duration}

The majority of trials were short term ( 2 to 12 weeks) with some medium term ( 13 to 26 weeks) and none long term ( $>26$ weeks). There was some evidence that the benefits of NRIs increase with a longer course of treatment. Therefore it is disappointing that so few studies followed patients up longer than a few weeks when the natural history of schizophrenia and likely duration of treatment would suggest much longer trials are needed to inform treatment decisions.

\section{Applicability}

\subsection{Patients}

The majority of trials included only patients with schizophrenia (mostly using DSM-IV criteria), limiting applicability to patients with other schizophrenia spectrum disorders such as schizoaffective disorder. As is usually the case with clinical trials most studies excluded patients with physical or psychiatric comorbidities, and substance misuse in particular, which somewhat limits applicability to real life clinical populations where comorbidity is common. The patient populations included in each study were relatively poorly characterised but overall it appeared that it was primarily patients with more chronic presentations.

Trials were conducted in a wide range of countries including Spain, South Africa, China, Israel, Iran, and the United States. While most outcomes were homogeneous it is noticeable that in those cases where there appeared to be benefits of NRIs over placebo, these frequently involved Chinese studies (Li 2008; Yu 2012; Zhao 2013), although this is also confounded, with studies specifically recruiting patients with negative symptoms or taking clozapine, 
and other studies recruiting patients with negative symptoms (e.g. Shafti 2015; Usall 2014), also showing trends for a benefit of NRIs.

\subsection{Dosage}

Most studies used doses of reboxetine up to $8 \mathrm{mg}$ daily with the rest using up to $4 \mathrm{mg}$ daily. However, $8 \mathrm{mg}$ daily is the starting dose of reboxetine for depression and it can be increased up to $12 \mathrm{mg}$ daily (BNF 2016), suggesting that it may have been under-dosed in these trials. The more positive Chinese studies used doses of $8 \mathrm{mg}$ daily.

Most trials using atomoxetine titrated up to $80 \mathrm{mg}$ daily, with one study using doses up to $120 \mathrm{mg}$ daily and one study including an arm with $40 \mathrm{mg}$ daily. When used for ADHD atomoxetine is started at $40 \mathrm{mg}$ daily, and increased to a usual maintenance dose of $80 \mathrm{mg}$ to $100 \mathrm{mg}$ daily, and can be increased up to $120 \mathrm{mg}$ daily (BNF 2016), again suggesting that most trials may have used doses at the lower end of the therapeutic range.

\subsection{Setting}

Where reported, most trials included only inpatients, and while most outcomes were not heterogeneous it is noticeable that in those cases where there appeared to be benefits of NRIs over placebo these frequently involved Chinese inpatients.

\section{Quality of the evidence}

For most domains of bias we judged the majority of trials to be of unclear or low risk; however in most domains there were also some trials at high risk of bias (see Figure 2; Figure 3). Although we included one open trial (Yu 2012), our sensitivity analyses did not suggest that this was driving the outcomes. We considered the quality of the current evidence to be low or very low using GRADE criteria, except for the findings of no effect of NRIs on CGI-S and PANSS positive score (Summary of findings for the main comparison); or all cause withdrawals, where we considered the evidence to be of moderate quality. The quality of evidence was downgraded due to small sample sizes with wide confidence intervals, poorly described methods, and evidence of selective reporting of outcomes and publication bias. There was a mixture of studies funded by pharmaceutical companies and by government agencies with no evidence of differential outcomes depending on funding source. There was a particular issue with skewed outcomes and we were unable to establish whether utilising change scores rather than endscores ameliorated this. We also made widespread utilisation of imputed SD. In our sensitivity analyses we did not detect evidence that skewed scores or imputed SD made a substantial difference to results.

\section{Potential biases in the review process}

\section{Unpublished data}

Our review has certainly identified the most studies of NRIs in schizophrenia to date. A number of the included studies were performed in China and reported in Chinese and had not previously been identified. This suggests that there may be a considerable literature available, published in regional non-English journals. There were also a number of studies which did not fully report their results or which appear to be completed but unpublished, suggesting that there is still a substantial amount of data that we have not been able to include in the review.

\section{Poor reporting and publication bias}

A number of studies did not provide useable data, often reporting no significant effect on these outcomes, which suggests the possibility of publication bias for positive studies. However, the vast majority of studies found no significant changes, partially due to small effect sizes resulting in low statistical power. The NRI literature is unusual in that most published studies have found negative results but more recently larger more positive studies have been published. It is possible that some smaller negative studies were not thought to warrant publication given the largely negative existing literature. Due to the limited number of studies, it was not possible to perform funnel plots to investigate publication bias.

\section{Chinese studies}

Three positive Chinese studies were major contributors to our review, driving many significant results (Li 2008; Yu 2012; Zhao 2013). There is some evidence that trial results from non-Western countries tend to over-report positive findings (Panagiotou 2013), particularly Chinese studies, and the most likely explanation for this is lower trial quality (Zhang 2011). We have not specifically conducted sensitivity analyses excluding these trials, preferring to rely on our assessments of risk on a trial-by-trial basis. Apart from the unblinded study by Yu 2012, the other two Chinese trials Li 2008 and Zhao 2013 - were not clearly of lower quality than the other studies in this review (Figure 3 ) but it is noticeable that reported standard deviations in all three trials were smaller than for the other trials included in this review and the random-effects model we used will only partially compensate for this.

\section{Agreements and disagreements with other studies or reviews}

We have reported effect sizes in terms of the underlying measurement scales in order to facilitate their interpretation. Paradoxically this makes comparisons with other studies difficult as these have mostly used the SMD as a measure of effect size. For illustrative purposes we have converted the short-term PANSS total scores and medium-term PANSS negative scores to the corresponding SMD using all change scores (as endscores and change scores cannot be combined when calculating the SMD). The PANSS total effect size goes from 2.8 to 2.4 points using change scores and this corresponds to an SMD of 0.4. Similarly the PANSS negative effect size goes from 3.3 to 3.7 points using change scores and this corresponds to an SMD of 1.1.

Rummel-Kluge 2006 conducted a Cochrane Review of antidepressants for negative symptoms in schizophrenia but did not include any selective noradrenaline reuptake inhibitors (NRIs), specifically excluding three studies as they did not recruit patients with predominantly negative symptoms (Kurland 1981; Poyurovsky 2003; Schutz 2001). Another Cochrane Review by Whitehead 2002 looked at the use of antidepressants in patients with comorbid schizophrenia and depression but the only trial of NRIs identified was Kurland 1981. Vernon 2014 conducted a review of antidepressants for cognitive symptoms in schizophrenia and found mostly non-significant or clinically unimportant effects and only identified one NRI study (Poyurovsky 2007). Singh 2010 conducted a meta-analysis looking at antidepressants for the treatment of the negative symptoms of schizophrenia. This found an overall benefit of antidepressants with an SMD around 0.5; but looking at three studies - Poyurovsky 2003, Poyurovsky 2007 and Schutz 2001 - concluded there was no benefit of reboxetine. 
They did not include atomoxetine as it is not considered an antidepressant. It is interesting that studies of mirtazapine and mianserin have found larger benefits than studies of SSRIs since the former have noradrenergic as well as serotonergic effects (Hecht 2012; Kishi 2014). We had only limited evidence to compare NRIs directly against SSRIs but two studies including reboxetine and citalopram arms found no statistically significant differences between them (Hinkelmann 2013; Usall 2014). However, neither study found a benefit of either citalopram or reboxetine over placebo and were not sufficiently powered to detect differences.

The most comparable study to our review is a meta-analysis by Kishi 2013 which updates Singh 2010 to look specifically at noradrenaline reuptake inhibitors. They included seven studies which are also included in our review (Ball 2011; Friedman 2008; Kelly 2009; Poyurovsky 2003; Poyurovsky 2007; Sacco 2009; Schutz 2001). They also included Poyurovsky 2013, which we excluded from our review as the intervention was reboxetine plus betahistine. In addition they included a trial of mazindol which they classified as a noradrenaline reuptake inhibitor but pointed out that it is known to additionally inhibit dopamine and serotonin reuptake. As our study looked specifically at selective noradrenaline reuptake inhibitors (NRIs) we made an a priori decision not to include mazindol. There were nine additional studies identified and included in our review that were not included in Kishi 2013 (Eli Lilly 2006; Ganguli 2008; Hinkelmann 2013; Kurland 1981; Li 2008; Shafti 2015; Usall 2014; Yu 2012; Zhao 2013). They are characterised mostly by having been published more recently or being published in Chinese. Kishi 2013 found no benefit of NRIs on overall, positive, or negative symptoms of schizophrenia with a borderline significant benefit on depressive symptoms. They also found no greater side-effects with noradrenaline reuptake-inhibitors except for an increased pulse rate and weight loss. Areas of overlap include the findings of NRIs benefiting depressive symptoms and decreasing weight gain and a general lack of side-effects for NRIs. Areas of difference include our finding of a benefit of NRIs on PANSS total scores in the short term (2 to 12 weeks) and PANSS negative scores in the medium term (13 to 26 weeks). It is notable that few of the studies contributing to these outcomes were included in Kishi 2013. The increased rate of dry mouth in our review was not analysed by Kishi 2013 and we did not look specifically at heart rate as a continuous outcome. They did not analyse cognitive outcomes, social functioning, or quality of life. We included viloxazine in our review as the limited data available about its pharmacological profile suggests it is selective for noradrenaline reuptake but it contributed few measures to our analysis.

Choi 2015 conducted a meta-analysis looking at medication for weight control in schizophrenia and identified two out of three studies included in our short-term analysis - Poyurovsky 2003 and Poyurovsky 2007 - finding a similar effect size. Mizuno 2014 also looked at medication for weight control and as well as the two studies included in Choi 2015 they also identified the Ball 2011 study of atomoxetine which showed no benefit on weight. Neither review identified Zhao 2013.

\section{AUTHORS' CONCLUSIONS}

\section{Implications for practice}

\section{For people with schizophrenia}

People with schizophrenia should know that there is not much evidence to draw conclusions about the effectiveness of NRIs in schizophrenia. What evidence there is suggests that NRIs, reboxetine in particular, added to antipsychotic medication might help with negative symptoms although this benefit is likely to be fairly small and may not be noticeable in real life. The main side-effects of NRIs are reduced weight gain, compared to taking antipsychotics alone, and dry mouth.

\section{For clinicians}

The available evidence from RCTs of NRIs used to augment antipsychotic medication provides tentative support for a benefit on negative symptoms that is fairly small in terms of absolute improvements on rating scales and is largely driven by trials from China mostly of inpatients taking clozapine. There was not sufficient evidence to usefully compare NRIs to SSRIs. Findings on individual quality of life and social functioning scales are interesting but far too unreliable to guide clinical practice. One noteworthy finding is that the positive symptoms of schizophrenia do not appear to be exacerbated by NRIs and this is of interest if, for example, use of atomoxetine was planned for the treatment of comorbid ADHD. The evidence does not show any benefit of NRIs for cognitive functioning in schizophrenia. Reboxetine reliably seems to attenuate antipsychotic-induced weight gain. The benefit is relatively small (around $2 \mathrm{~kg}$ on average in the short term; and $7 \mathrm{~kg}$ in the medium term) but does highlight that the side-effects of reboxetine do not usually include weight gain in contrast to many other strategies for augmenting antipsychotic treatment. NRIs appear generally well tolerated with the main identified sideeffect being dry mouth.

\section{For policy makers}

Policy makers should support further trials into potential treatments for the more difficult to treat, but likely more disabling, negative and cognitive symptoms of schizophrenia. They should also mandate where possible the comprehensive publication of research findings needed to fully assess the totality of evidence for any given treatment.

\section{Implications for research}

\section{General}

The quality of reporting of clinical trials remains poor and there is evidence that many have not been published. Outcomes continue to be reported selectively and often not in a useable form for meta-analysis. There is also poor reporting of adverse effects and withdrawals. We have written to the corresponding authors of included and ongoing studies but have received few responses. This is a significant failure in the clinical trials process which has not been addressed by the pre-registration of trials, and represents a waste of financial investment and clinical knowledge. Following CONSORT standards (Moher 2001) and making data available for future researchers (AllTrials) would go a long way to addressing this.

\section{Specific}

\subsection{For reviews}

Several of the included and excluded studies could also be relevant for other existing Cochrane Schizophrenia Group reviews (Table 12). In particular this suggests the need for a review of serotonergic antidepressant augmentation in schizophrenia that does not just focus on those with negative symptoms. 


\subsection{For trials}

The evidence base for NRIs in schizophrenia has developed from a series of relatively small studies showing little benefit to increasingly larger studies showing more significant effects. Given the accumulating but equivocal evidence for a benefit of NRIs in schizophrenia, further larger RCTs are clearly needed. There was evidence from our results that treatment in the medium term leads to greater benefits from NRIs, suggesting that longer trials are indicated. There were also hints that recruiting specifically patients with prominent negative symptoms or on clozapine could also magnify the benefit. Although in many ways reboxetine is a drug looking for an indication in our review, the evidence was more supportive of reboxetine than atomoxetine. While the results of cognitive testing were generally negative these were all short-term and usually involved atomoxetine rather than reboxetine. A larger RCT (with hundreds rather than tens of patients) investigating the benefit of reboxetine on negative symptoms and cognitive functioning over a longer period (at least 3 months) would be most informative, particularly if including patients with prominent negative symptoms and using higher doses (at least $8 \mathrm{mg}$ daily). See Table 13 for a suggested design for a future RCT.

\section{ACKNOWLEDGEMENTS}

The Cochrane Schizophrenia Group (CSG) Editorial Base in Nottingham produces and maintains standard text for use in the Methods section of their reviews. We have used this text as the basis of what appears here and adapted it as required. Our thanks to Clive Adams and Claire Irving for providing editorial support and advice and particular thanks to Farhad Shokraneh for his help and advice in designing the search strategy and Clive Adams for extensive assistance in revising the final text.

We would like to thank Dr Qi Pei for her assistance with Chinese translations and checking the accuracy of data extraction from Chinese papers.

We are grateful to those authors who responded to enquiries to provide additional data or to clarify methods. 


\section{R E F E R E N C E S}

\section{References to studies included in this review}

Ball 2011 \{published data only (unpublished sought but not used)\} Ball MP, Warren KR, Feldman S, McMahon RP, Kelly DL, Buchanan RW. Placebo-controlled trial of atomoxetine for weight reduction in people with schizophrenia treated with clozapine or olanzapine. Clinical Schizophrenia \& Related Psychoses 2011;5(1):17-25.

NCT00176436. Double-blind study of atomoxetine for weight management in patients taking olanzapine or clozapine. www.clinicaltrials.gov (first received 2005).

Eli Lilly 2006 \{unpublished data only\}

NCT00089869. A combination of two currently approved drugs to enhance the treatment of schizophrenia. www.clinicaltrials.gov (first received 2004).

Friedman 2008 \{published data only (unpublished sought but not used)\}

Friedman JI, Carpenter D, Lu J, Fan J, Tang CY, White L, et al. A pilot study of adjunctive atomoxetine treatment to second-generation antipsychotics for cognitive impairment in schizophrenia. Journal of Clinical Psychopharmacology 2008;28(1):59-63. [MEDLINE: 18204342]

NCT00488163. Pilot study of atomoxetine to enhance cognition in patients with schizophrenia. www.clinicaltrials.gov (first received 2007)

\section{Ganguli 2008 \{published data only (unpublished sought but not used)\}}

Ganguli R. Atomoxetine for schizophrenia. Stanley Foundation Research Programs 2009.

Ganguli R, Brar J. Effect of atomoxetine on negative symptoms of schizophrenia: a randomized double-blind placebocontrolled clinical trial. Schizophrenia Research 2008;1-3(Suppl 2):254.

NCT00222794. A randomized, parallel group, double blind, placebo controlled, clinical trial of augmentation with atomoxetine for the treatment of negative symptoms in patients with schizophrenia and schizoaffective disorder. www.clinicaltrials.gov (first received 2005).

Hinkelmann 2013 \{published data only (unpublished sought but not used)\}

Hinkelmann K, Yassouridis A, Kellner M, Jahn H, Wiedemann K, Raedler TJ. No effects of antidepressants on negative symptoms in schizophrenia. Journal of Clinical Psychopharmacology 2013;33(5):686-90. [CRS: 9000120000000058$]$

Kelly 2009 \{published data only (unpublished sought but not used)\} Conley R. Double-blind, placebo-controlled, randomized study of atomoxetine for schizophrenia. Stanley Foundation Research Programs 2009.

Kelly DL, Buchanan RW, Boggs DL, McMahon RP, Dickinson D, Nelson $\mathrm{M}$, et al. A randomized double-blind trial of atomoxetine for cognitive impairments in 32 people with schizophrenia. Journal of Clinical Psychiatry 2009;70(4):518-25. [MEDLINE: 19358788]

NCT00161031. Atomoxetine as an adjunct to novel antipsychotic medication in the treatment of cognitive deficits of schizophrenia. www.clinicaltrials.gov (first accessed 2005).

Kurland 1981 \{published data only (unpublished sought but not used)\}

Kurland AA, Nagaraju A. Viloxazine and the depressed schizophrenic - methodological issues. Journal of Clinical Pharmacology 1981;21(1):37-41. [MEDLINE: 7012190]

Li 2008 \{published data only (unpublished sought but not used)\}

Li X, Wang Z, Zhao S. Effect of reboxetine combined with clozapine on negative symptoms quality of life of the patients with schizophrenia [瑞波西汀合并氯氮平对精神分裂症患 者阴性症状和生活质量的影响]. Chinese Journal of Health Psychology [\#\#\#\#\#\#\#\#] 2008;16(2):133-5.

李喜泼, 王占敏, 赵树霞, 黄秀芳, 许小梅, 钮伟芳, et al. Reboxetine combined clozapine on negative symptoms and social function in schizophrenia [瑞波西汀合并氯氮平对精神 分裂症患者阴性症状和社会功能的影响]. Journal of Practical Medicine [\#\#\#\#\#] 2008;24(3):445-6.

Poyurovsky 2003 \{published data only (unpublished sought but not used)\}

Poyurovsky M, Isaacs I, Fuchs C, Schneidman M, Faragian S, Weizman RWA. Attenuation of olanzapine-induced weight gain with reboxetine in patients with schizophrenia: a doubleblind, placebo-controlled study. American Journal of Psychiatry 2003;160(2):297-302. [MEDLINE: 12562576]

Poyurovsky M, Isaaks I, Fuchs C, Schneidman M, Faragian S, Weizman R, et al. Reboxetine and attenuation of olanzapineinduced weight gain in first-episode schizophrenia patients. A double-blind placebo-controlled study. International Journal of Neuropsychopharmacology 2002;5(Suppl 1):S171.

Poyurovsky M, Isaaks I, Pashnian A, Fuchs C, Schneidman M, Faragian S, et al. Reboxetine but not fluoxetine attenuates olanzapine-induced weight gain in first-episode schizophrenia patients. European Neuropsychopharmacology 2002;12(Suppl 3):S312.

Poyurovsky M, Maayan R, Gil-Ad I, Weizman A, Weizman R. Reboxetine attenuates weight gain and increases dehydroepiandrosterone levels in olanzapine- treated schizophrenia patients. 156th Annual Meeting of the American Psychiatric Association; 2003 May 17-22; San Francisco, California, USA. 2003.

Poyurovsky 2007 \{published data only (unpublished sought but not used)\}

Poyurovsky M. Reboxetine for schizophrenia. Stanley Foundation Research Programs 2009.

Poyurovsky M, Faragian S, Fuchs C, Pashinian A. Effect of the selective norepinephrine reuptake inhibitor reboxetine 
on cognitive dysfunction in schizophrenia patients: an addon, double-blind placebo-controlled study. Israel Journal of Psychiatry and Related Sciences 2009;46(3):213-20. [MEDLINE: 20039523]

Poyurovsky M, Fuchs C, Pashinian A, Levi A, Faragian S, Maayan $\mathrm{R}$, et al. Attenuating effect of reboxetine on appetite and weight gain in olanzapine-treated schizophrenia patients: a double-blind placebo-controlled study. 62nd Annual Scientific Meeting of the Society of Biological Psychiatry; 2007 May 17-19; San Diego (CA). San Diego, CA: Elsevier Science Inc, 2007.

Poyurovsky M, Fuchs C, Pashinian A, Levi A, Faragian S, Maayan $\mathrm{R}$, et al. Attenuating effect of reboxetine on appetite and weight gain in olanzapine-treated schizophrenia patients: a double-blind placebo-controlled study. Psychopharmacology 2007;192(3):441-8. [MEDLINE: 17310385]

Sacco 2009 \{published data only (unpublished sought but not used)\}

NCT00420498. Effects of atomoxetine on cognitive function in schizophrenia. www.clinicaltrials.gov (first received 2007).

Sacco KA, Creeden C, Reutenauer EL, Vessicchio JC, Weinberger AH, George TP. Effects of atomoxetine on cognitive function and cigarette smoking in schizophrenia. Schizophrenia Research 2009;107(2-3):332-3.

Schutz 2001 \{published data only (unpublished sought but not used)\}

Schutz G, Berk M. Reboxetine add on therapy to haloperidol in the treatment of schizophrenia: a preliminary double-blind randomized placebo-controlled study. International Clinical Psychopharmacology 2001;16(5):275-8. [MEDLINE: 11552770]

Shafti 2015 \{published data only (unpublished sought but not used)\}

Shafti SS, Jafarabad MS, Azizi R. Amelioration of deficit syndrome of schizophrenia by norepinephrine reuptake inhibitor. Therapeutic Advances in Psychopharmacology 2015; Vol. 5, issue 5:263-70.

Usall 2014 \{published data only (unpublished sought but not used)\} NCT01300364. Reboxetine and citalopram as an adjunct treatment to second generation antipsychotics in the treatment of negative symptoms of schizophrenia. ClinicalTrials.gov/ show/NCT01300364 (first received 2011).

Usall J, López-Carrilero R, Iniesta R, Roca M, Caballero M, Rodriguez-Jimenez R, et al. Double-blind, placebo-controlled study of the efficacy of reboxetine and citalopram as adjuncts to atypical antipsychotics for negative symptoms of schizophrenia. Journal of Clinical Psychiatry 2014;75(6):608-15.

Yu 2012 \{published data only (unpublished sought but not used)\} 于晶妮, 柴萌. Olanzapine combined with reboxetine for treatment of negative symptoms of schizophrenia: a controlled study. 吉林医学 2012; Vol. 23:4988-9.

Zhao 2013 \{published data only (unpublished sought but not used)\} 赵树霞, 李占胜, 耿寒松, 尹秀茹, 刘亚丽, 崔慧敏, et al. Reboxetine treatment of clozapine-induced metabolic syndrome randomized double-blind controlled study. Hebei Medical Journal [河北医药] 2013, issue 21:3226-8.

\section{References to studies excluded from this review}

Amrami-Weizman 2013 \{published data only\}

Amrami-Weizman A, Maayan R, Gil-Ad I, Pashinian A, Fuchs C, Kotler $\mathrm{M}$, et al. The effect of reboxetine co-administration with olanzapine on metabolic and endocrine profile in schizophrenia patients. Psychopharmacology 2013;230(1):23-7. [CRS: 9000120000001098]

\section{Apud 2007a $\{$ published data only\}}

NCT00548327. Randomized, double-blinded, placebo controlled study of the effects of atomoxetine on cognitive function in patients with schizophrenia and normal controls based on comt genotype. www.clinicaltrials.gov (first received 2007).

\section{Barnes 2009 \{published data only\}}

Barnes T. Antidepressant controlled trial for negative symptoms in schizophrenia (ACTIONS). National Institute for Health Research 2009.

ISRCTN42305247. Antidepressant controlled trial for negative symptoms in schizophrenia. www.controlled-trials.com (first received 2010).

NCT01032083. Antidepressant controlled trial for negative symptoms in schizophrenia. www.clinicaltrials.gov (first received 2009).

Hou 2007 \{published data only\}

Hou HY, Zhang SL, Zhang ZA. The efficacy of haloperidol combined with xitelopram in the treatment of schizophrenia with predominantly negative symptoms [氟哌啶醇并西肽普兰 治疗以阴性症状为主的精神分裂症的效果]. Medical Journal of Qilu 2007;22(6):504-5.

\section{Mueller 2005 \{published data only\}}

Mueller N, Riedel M, Dehning S, Spellmann I, Mueller-Arends A, Cerovecki A, et al. Cox-2 inhibition in schizophrenia and major depression - the advent of immunotherapy in psychiatry?. 8th World Congress of Psychiatry; 2005 Sep 10-15; Cairo, Egypt. 2005.

\section{Poyurovsky 2013 \{published data only\}}

Poyurovsky M, Fuchs C, Pashinian A, Levi A, Weizman R, Weizman A. Reducing antipsychotic-induced weight gain in schizophrenia: a double-blind placebo-controlled study of reboxetine-betahistine combination. Psychopharmacology 2013;226(3):615-22. [CRS: 9000102000000308; PUBMED: 23239133]

\section{Salokangas 1997 \{published data only\}}

Salokangas RK, Saarijarvi S, Taiminen T, Lehto H, Niemi H, Ahola V, et al. Effect of smoking on neuroleptics in schizophrenia. Schizophrenia Research 1997;23(1):55-60. [MEDLINE: 9050128] 
Shafti 2004 \{published data only\}

Shoja Shafti S. Effectiveness of citalopram, alprazolam and clomipramine in ameliorate of negative symptoms in schizophrenia. Journal of Rehabilitation 2004;3(12):42-9. [CRS: 9000100000006555]

Shoja Shafti S. Rehabilitation of schizophrenia: adjunctive therapy of negative symptoms. Iranian Rehabilitation Journal 2004;2(2):15-25.

\section{References to studies awaiting assessment}

Shekhar 2005 \{published data only (unpublished sought but not used)\}

NCT00216281. Correlation of phenotype, genotype and clinical efficacy/toxicity of clozapine augmented by atomoxetine for treatment refractory schizophrenia (CAPG study). www.clinicaltrials.gov (first received 2005).

Tamminga 2009 \{published data only (unpublished sought but not used)\}

NCT00628394. Treating cognition in schizophrenia with atomoxetine and cognitive remediation. www.clinicaltrials.gov (first received 2008).

Shad MU, Moore D, Shepard M, Tamminga C. Pharmacological treatment of insight deficits in schizophrenia. Biological Psychiatry 2010;67:264S.

Stan AD, Bushong D, Thomas B. Cognitive training with pharmacological enhancement in schizophrenia. Neuropsychopharmacology 2013;38(Suppl 1):S524. [CRS: 9000120000002385]

Tamminga C. Atomoxetine for schizophrenia. Stanley Foundation Research Programs 2009.

\section{References to ongoing studies}

Baranchik 2006 \{published data only (unpublished sought but not used)\}

NCT00409201. Phase 1 study of reboxetine adjuvant therapy and cognitive and behavioral measures. www.clinicaltrials.gov (first received 2006).

\section{Additional references}

\section{Akhondzadeh 2011}

Akhondzadeh S, Modabbernia A, Taslimi S, Daneshmand A, Ostovaneh M. Celecoxib for schizophrenia. Cochrane Database of Systematic Reviews 2011, Issue 7. [DOI: 10.1002/14651858.CD009205]

\section{AllTrials}

AllTrials. All Trials Registered | All Results Reported. www.alltrials.net (accessed 6 March 2017).

\section{Altman 1996 \\ Altman DG, Bland JM. Detecting skewness from summary information. BMJ 1996;313(7066):1200.}

\section{Andreasen 1982}

Andreasen NC, Olsen S. Negative v positive schizophrenia. Definition and validation. Archive of General Psychiatry 1982;39(7):789-94. [PUBMED: 7165478]

\section{Andreasen 1984}

Andreasen NC. The Scale for the Assessment of Positive Symptoms (SAPS). Iowa City, IA: The University of lowa, 1984.

\section{Apud 2007b}

Apud JA, Weinberger DR. Treatment of cognitive deficits associated with schizophrenia: potential role of catechol-0methyltransferase inhibitors. CNS drugs 2007;21(7):535-57. [PUBMED: 17579498]

\section{Barnes 1989}

Barnes TR. A rating scale for drug-induced akathisia. British Journal of Psychiatry 1989;154:672-6. [PUBMED: 2574607]

\section{Barnes 2011}

Barnes TR, Schizophrenia Consensus Group of British Association for Psychopharmacology. Evidence-based guidelines for the pharmacological treatment of schizophrenia: recommendations from the British Association for Psychopharmacology. Journal of Psychopharmacology 2011;25(5):567-620. [PUBMED: 21292923]

\section{Bilder 2000}

Bilder RM, Goldman RS, Robinson D, Reiter G, Bell L, Bates JA, et al. Neuropsychology of first-episode schizophrenia: initial characterization and clinical correlates. American Journal of Psychiatry 2000;157(4):549-59. [PUBMED: 10739413]

\section{Bland 1997}

Bland JM. Statistics notes. Trials randomised in clusters. BMJ 1997;315:600.

\section{BNF 2016}

Joint Formulary Committee. British National Formulary. www.bnf.org. London: BMJ Group and Pharmaceutical Press, (accessed 12 May 2016).

\section{Boissel 1999}

Boissel JP, Cucherat M, Li W, Chatellier G, Gueyffier F, Buyse $M$, et al. The problem of therapeutic efficacy indices. 3. Comparison of the indices and their use [Apercu sur la problematique des indices d'efficacite therapeutique, 3 : comparaison des indices et utilisation. Groupe d'etude des Indices d'efficacite]. Therapie 1999;54(4):405-11. [PUBMED: 10667106]

\section{Bymaster 2002}

Bymaster FP, Katner JS, Nelson DL, Hemrick-Luecke SK, Threlkeld PG, Heiligenstein JH, et al. Atomoxetine increases extracellular levels of norepinephrine and dopamine in prefrontal cortex of rat: a potential mechanism for efficacy in attention deficit/hyperactivity disorder. Neuropsychopharmacology 2002;27(5):699-711. [PUBMED: 12431845] 


\section{Chamberlain 2007}

Chamberlain SR, Del Campo N, Dowson J, Muller U, Clark L, Robbins TW, et al. Atomoxetine improved response inhibition in adults with attention deficit/hyperactivity disorder. Biological Psychiatry 2007;62(9):977-84. [PUBMED: 17644072]

\section{Choi 2015}

Choi YJ. Efficacy of adjunctive treatments added to olanzapine or clozapine for weight control in patients with schizophrenia: a systematic review and meta-analysis. Scientific World Journal 2015;2015:970730. [PUBMED: 25664341]

\section{Cipriani 2009}

Cipriani A, Furukawa TA, Salanti G, Geddes JR, Higgins JP, Churchill R, et al. Comparative efficacy and acceptability of 12 new-generation antidepressants: a multiple-treatments metaanalysis. Lancet 2009;373(9665):746-58. [PUBMED: 19185342]

\section{Crow 1980}

Crow TJ. Molecular pathology of schizophrenia: more than one disease process?. British Medical Journal 1980;280(6207):66-8. [PUBMED: 6101544]

\section{Daniel 1991}

Daniel DG, Weinberger DR, Jones DW, Zigun JR, Coppola R, Handel $S$, et al. The effect of amphetamine on regional cerebral blood flow during cognitive activation in schizophrenia. Journal of Neuroscience 1991;11(7):1907-17. [PUBMED: 2066768]

\section{Deeks 2000}

Deeks J. Issues in the selection for meta-analyses of binary data. 8th International Cochrane Colloquium; 2000 Oct 25-28; Cape Town. Cape Town: The Cochrane Collaboration, 2000.

\section{Divine 1992}

Divine GW, Brown JT, Frazier LM. The unit of analysis error in studies about physicians' patient care behavior. Journal of General Internal Medicine 1992;7(6):623-9.

\section{Donner 2002}

Donner A, Klar N. Issues in the meta-analysis of cluster randomized trials. Statistics in Medicine 2002;21(19):2971-80.

\section{Egger 1997}

Egger M, Davey Smith G, Schneider M, Minder C. Bias in meta-analysis detected by a simple, graphical test. BMJ 1997;315(7109):629-34.

\section{Elbourne 2002}

Elbourne D, Altman DG, Higgins JPT, Curtina F, Worthingtond HV, Vaile A. Meta-analyses involving crossover trials: methodological issues. International Journal of Epidemiology 2002;31(1):140-9.

\section{Eli Lilly 2015}

Eli Lilly. Summary of Product Characteristics Strattera 10mg, $18 \mathrm{mg}, 25 \mathrm{mg}$, $40 \mathrm{mg}$, $60 \mathrm{mg}$, $80 \mathrm{mg}$ or $100 \mathrm{mg}$ hard capsules. http://www.medicines.org.uk Accessed 20th September 2017.

\section{Eyding 2010}

Eyding D, Lelgemann M, Grouven U, Harter M, Kromp M, Kaiser T, et al. Reboxetine for acute treatment of major depression: systematic review and meta-analysis of published and unpublished placebo and selective serotonin reuptake inhibitor controlled trials. BMJ 2010;341:c4737. [PUBMED: 20940209]

\section{Faraone 2005}

Faraone SV, Biederman J, Spencer T, Michelson D, Adler L, Reimherr F, et al. Atomoxetine and stroop task performance in adult attention-deficit/hyperactivity disorder. Journal of Child and Adolescent Psychopharmacology 2005;15(4):664-70. [PUBMED: 16190797]

\section{Fleurence 2009}

Fleurence R, Williamson R, Jing Y, Kim E, Tran QV, Pikalov AS, et al. A systematic review of augmentation strategies for patients with major depressive disorder. Psychopharmacology Bulletin 2009;42(3):57-90. [PUBMED: 19752841]

\section{Friedman 1999}

Friedman JI, Temporini H, Davis KL. Pharmacologic strategies for augmenting cognitive performance in schizophrenia. Biological Psychiatry 1999;45(1):1-16. [PUBMED: 9894570]

\section{Furukawa 2006}

Furukawa TA, Barbui C, Cipriani A, Brambilla P, Watanabe N. Imputing missing standard deviations in meta-analyses can provide accurate results. Journal of Clinical Epidemiology 2006;59(1):7-10.

\section{Goldberg 2007}

Goldberg TE, Goldman RS, Burdick KE, Malhotra AK, Lencz T, Patel RC, et al. Cognitive improvement after treatment with second-generation antipsychotic medications in first-episode schizophrenia: is it a practice effect?. Archives of General Psychiatry 2007;64(10):1115-22. [PUBMED: 17909123]

\section{Green 1996}

Green MF. What are the functional consequences of neurocognitive deficits in schizophrenia?. American Journal of Psychiatry 1996;153(3):321-30. [PUBMED: 8610818]

\section{Gulliford 1999}

Gulliford MC. Components of variance and intraclass correlations for the design of community-based surveys and intervention studies: data from the Health Survey for England 1994. American Journal of Epidemiology 1999;149(9):876-83.

\section{Guo 1995}

Guo GY. Reliability and validity of the scale of social skills of chronic schizophrenic in-patients. Chinese Journal of Neurology 1995;28:16-8.

\section{Guy 1976}

Guy W. ECDEU Assessment Manual for Psychopharmacology. Rockville, MD: US Department of Heath, Education, and Welfare Public Health Service Alcohol, Drug Abuse, and Mental Health Administration, 1976. 


\section{Hamilton 1980}

Hamilton M. Rating depressive patients. Journal of Clinical Psychiatry 1980;41(12 Pt 2):21-4. [PUBMED: 7440521]

\section{Hecht 2012}

Hecht EM, Landy DC. Alpha-2 receptor antagonist add-on therapy in the treatment of schizophrenia: a meta-analysis. Schizophrenia Research 2012;134(2-3):202-6. [PUBMED: 22169246]

\section{Higgins 2003}

Higgins JPT, Thompson SG, Deeks JJ, Altman DG. Measuring inconsistency in meta-analyses. BMJ 2003;327(7414):557-60.

\section{Higgins 2011}

Higgins JPT, Green S (editors). Cochrane Handbook for Systematic Reviews of Interventions Version 5.1.0 [updated March 2011]. The Cochrane Collaboration, 2011. Available from handbook.cochrane.org.

\section{Jasinski 2008}

Jasinski DR, Faries DE, Moore RJ, Schuh LM, Allen AJ. Abuse liability assessment of atomoxetine in a drug-abusing population. Drug and Alcohol Dependence 2008;95(1-2):140-6. [PUBMED: 18328639]

\section{Kay 1986}

Kay SR, Opler LA, Fiszbein A. Positive and Negative Syndrome Scale (PANSS) Manual. North Tonawanda, NY: Multi-Health Systems, 1986.

\section{Kishi 2013}

Kishi T, Mukai T, Matsuda Y, Moriwaki M, Iwata N. Efficacy and safety of noradrenalin reuptake inhibitor augmentation therapy for schizophrenia: a meta-analysis of double-blind randomized placebo-controlled trials. Journal of Psychiatric Research 2013;47(11):1557-63. [PUBMED: 23899496]

\section{Kishi 2014}

Kishi T, Iwata N. Meta-analysis of noradrenergic and specific serotonergic antidepressant use in schizophrenia. International Journal of Neuropsychopharmacology 2014;17(2):343-54. [PUBMED: 23823741]

\section{Knable 1997}

Knable MB, Weinberger DR. Dopamine, the prefrontal cortex and schizophrenia. Journal of Psychopharmacology 1997;11(2):123-31. [PUBMED: 9208376]

\section{Leucht 2005a}

Leucht S, Kane JM, Kissling W, Hamann J, Etschel E, Engel RR. What does the PANSS mean?. Schizophrenia Research 2005;79(2-3):231-8. [PUBMED: 15982856]

\section{Leucht 2005b}

Leucht S, Kane JM, Kissling W, Hamann J, Etschel E, Engel R. Clinical implications of brief psychiatric rating scale scores. British Journal of Psychiatry 2005;187:366-71. [PUBMED: 16199797]

\section{Leucht 2007}

Leucht S, Engel RR, Bauml J, Davis JM. Is the superior efficacy of new generation antipsychotics an artifact of LOCF?. Schizophrenia Bulletin 2007;33(1):183-91. [PUBMED: 16905632]

\section{Leucht 2009}

Leucht S, Corves C, Arbter D, Engel RR, Li C, Davis JM. Secondgeneration versus first-generation antipsychotic drugs for schizophrenia: a meta-analysis. Lancet 2009;373(9657):31-41. [PUBMED: 19058842]

\section{Levine 2013}

Levine SZ, Leucht S. Identifying clinically meaningful symptom response cut-off values on the SANS in predominant negative symptoms. Schizophrenia Research 2013;145(1-3):125-7. [PUBMED: 23394742]

\section{Lopez-Munoz 2009}

Lopez-Munoz F, Alamo C. Monoaminergic neurotransmission: the history of the discovery of antidepressants from 1950s until today. Current Pharmaceutical Design 2009;15(14):1563-86. [PUBMED: 19442174]

\section{Lu 2007}

Lu W, Cui Y, Zheng Y, Gu K, Cai H, Li Q, et al. Impact of newly diagnosed breast cancer on quality of life among Chinese women. Breast Cancer Research Treatment 2007;102(2):201-10. [PUBMED: 16850242]

\section{Makinen 2008}

Makinen J, Miettunen J, Isohanni M, Koponen H. Negative symptoms in schizophrenia: a review. Nordic Journal of Psychiatry 2008;62(5):334-41. [PUBMED: 18752104]

\section{Marcus 2010}

Marcus MM, Jardemark K, Malmerfelt A, Bjorkholm C, Svensson TH. Reboxetine enhances the olanzapine-induced antipsychotic-like effect, cortical dopamine outflow and NMDA receptor-mediated transmission. Neuropsychopharmacology 2010;35(9):1952-61. [PUBMED: 20463659]

\section{Marin-Martinez 1999}

Marin-Martinez F, Sanchez-Meca J. Averaging dependent effect sizes in meta-analysis: a cautionary note about procedures. Spanish Journal of Psychology 1999;2(1):32-8. [PUBMED: 11757259]

\section{Marshall 2000}

Marshall M, Lockwood A, Bradley C, Adams C, Joy C, Fenton M. Unpublished rating scales: a major source of bias in randomised controlled trials of treatments for schizophrenia. British Journal of Psychiatry 2000;176:249-52.

\section{Masana 2011}

Masana M, Bortolozzi A, Artigas F. Selective enhancement of mesocortical dopaminergic transmission by noradrenergic drugs: therapeutic opportunities in schizophrenia. The International Journal of Neuropsychopharmacology 2011;14(1):53-68. [PUBMED: 20701825] 


\section{Maudsley 2015}

Taylor D, Paton C, Kapur S. The Maudsley Prescribing Guidelines in Psychiatry. 12th Edition. Wiley-Blackwell, 2015.

\section{Mizuno 2014}

Mizuno Y, Suzuki T, Nakagawa A, Yoshida K, Mimura M, Fleischhacker WW, et al. Pharmacological strategies to counteract antipsychotic-induced weight gain and metabolic adverse effects in schizophrenia: a systematic review and metaanalysis. Schizophrenia Bulletin 2014;40(6):1385-403. [PUBMED: 24636967]

\section{Moher 2001}

Moher D, Schulz KF, Altman D, CONSORT Group (Consolidated Standards of Reporting Trials). The CONSORT statement: revised recommendations for improving the quality of reports of parallel-group randomized trials. JAMA 2001;285(15):1987-91.. [PUBMED: 11308435]

\section{Munetz 1988}

Munetz MR, Benjamin S. How to examine patients using the Abnormal Involuntary Movement Scale. Hospital Community Psychiatry 1988;39(11):1172-7. [PUBMED: 2906320]

\section{Nolte 2004}

Nolte S, Wong D, Lachford G. Amphetamines for schizophrenia. Cochrane Database of Systematic Reviews 2004, Issue 4. [DOI: 10.1002/14651858.CD004964; PUBMED: 15495131]

\section{Nuechterlein 2004}

Nuechterlein KH, Barch DM, Gold JM, Goldberg TE, Green MF, Heaton RK. Identification of separable cognitive factors in schizophrenia. Schizophrenia Research 2004;72(1):29-39. [PUBMED: 15531405]

\section{Overall 1962}

Overall JE, Gorham DR. The brief psychiatric rating scale. Psychological Reports 1962;10:799-812.

\section{Panagiotou 2013}

Panagiotou OA, Contopoulos-Ioannidis DG, Ioannidis JP. Comparative effect sizes in randomised trials from less developed and more developed countries: metaepidemiological assessment. BMJ 2013;346:f707. [PUBMED: 23403829]

\section{Pfizer 2015}

Pfizer. Summary of Product Characteristics Edronax $4 \mathrm{mg}$ Tablets. https://www.medicines.org.uk Accessed 20th September 2017.

\section{Preti 2002}

Preti A. Tomoxetine (Eli Lilly \& Co). Current Opinion in Investigational Drugs 2002;3(2):272-7. [PUBMED: 12020059]

\section{Prudo 1987}

Prudo R, Blum HM. Five-year outcome and prognosis in schizophrenia: a report from the London field research centre of the international pilot study of schizophrenia. British Journal of Psychiatry 1987;150:345-54. [PUBMED: 3664104]

\section{Rabinowitz 2006}

Rabinowitz J, Mehnert A, Eerdekens M. To what extent do the PANSS and CGI-S overlap?. Journal of Clinical Psychopharmacology 2006;26(3):303-7. [PUBMED: 16702895]

\section{Raedler 2004}

Raedler TJ, Jahn H, Arlt J, Kiefer F, Schick M, Naber D, et al. Adjunctive use of reboxetine in schizophrenia. European Psychiatry 2004;19(6):366-9. [PUBMED: 15363476]

\section{Rummel-Kluge 2006}

Rummel-Kluge C, Kissling W, Leucht S. Antidepressants for the negative symptoms of schizophrenia. Cochrane Database of Systematic Reviews 2006, Issue 3. [DOI: 10.1002/14651858.CD005581.pub2; PUBMED: 16856105]

\section{Schneider 1983}

Schneider LC, Struening EL. SLOF: a behavioral rating scale for assessing the mentally ill. Social Work Research and Abstracts 1983;19(3):9-21. [PUBMED: 10264257]

\section{Schünemann 2008}

Schünemann HJ, Oxman AD, Vist GE, Higgins JPT, Deeks JJ, Glasziou P, et al. Chapter 12: Interpreting results and drawing conclusions. In: Higgins JPT, Green S (editors), Cochrane Handbook for Systematic Reviews of Interventions Version 5.1.0 (updated March 2011). The Cochrane Collaboration, 2011. Available from handbook.cochrane.org.

\section{Seeman 1975}

Seeman P, Chau-Wong M, Tedesco J, Wong K. Brain receptors for antipsychotic drugs and dopamine: direct binding assays. National Academy of Sciences of the United States of America 1975;72(11):4376-80. [PUBMED: 1060115]

\section{Seu 2009}

Seu E, Lang A, Rivera R, Jentsch JD. Inhibition of the norepinephrine transporter improves behavioral flexibility in rats and monkeys. Psychopharmacology 2009;202(1-3):505-19.

\section{Simpson 1970}

Simpson GM, Angus JW. A rating scale for extrapyramidal side effects. Acta Psychiatrica Scandinavica Supplementum 1970;212:11-9. [PUBMED: 4917967]

\section{Singh 2010}

Singh SP, Singh V, Kar N, Chan K. Efficacy of antidepressants in treating the negative symptoms of chronic schizophrenia: meta-analysis. British Journal of Psychiatry 2010;197(3):174-9. [PUBMED: 20807960]

\section{Sterne 2011}

Sterne JA, Sutton AJ, Ioannidis JP, Terrin N, Jones DR, Lau J, et al. Recommendations for examining and interpreting funnel plot asymmetry in meta-analyses of randomised controlled trials. BMJ 2011;343:d4002. [PUBMED: 21784880]

\section{Tiihonen 2009}

Tiihonen J, Wahlbeck K, Kiviniemi V. The efficacy of lamotrigine in clozapine-resistant schizophrenia: a systematic review and 
meta-analysis. Schizophrenia Research 2009;109(1-3):10-14. [PUBMED: 19186030]

\section{Ukoumunne 1999}

Ukoumunne OC, Gulliford MC, Chinn S, Sterne JAC, Burney PG. Methods for evaluating area-wide and organisation-based intervention in health and health care: a systematic review. Health Technology Assessment 1999;3(5):iii-92.

\section{Vernon 2014}

Vernon JA, Grudnikoff E, Seidman AJ, Frazier TW, Vemulapalli MS, Pareek P, et al. Antidepressants for cognitive impairment in schizophrenia - a systematic review and metaanalysis. Schizophrenia Research 2014;159(2-3):385-94.

[PUBMED: 25240772]

\section{Weickert 2000}

Weickert TW, Goldberg TE, Gold JM, Bigelow LB, Egan MF, Weinberger DR. Cognitive impairments in patients with schizophrenia displaying preserved and compromised intellect. Archives of General Psychiatry 2000;57(9):907-13. [PUBMED: 10986554]

\section{Whitehead 2002}

Whitehead C, Moss S, Cardno A, Lewis G. Antidepressants for people with both schizophrenia and depression.

\section{CHARACTERISTICS OF STUDIES}

Characteristics of included studies [ordered by study ID]
Cochrane Database of Systematic Reviews 2002, Issue 2. [DOI: 10.1002/14651858.CD002305; PUBMED: 12076447]

\section{Wykes 2011}

Wykes T, Huddy V, Cellard C, McGurk SR, Czobor P. A metaanalysis of cognitive remediation for schizophrenia: methodology and effect sizes. American Journal of Psychiatry 2011;168(5):472-85. [PUBMED: 21406461]

\section{Xia 2009}

Xia J, Adams CE, Bhagat N, Bhagat V, Bhoopathi P, El-Sayeh H, et al. Loss to outcomes stakeholder survey: the LOSS study. Psychiatric Bulletin 2009;33(7):254-7.

\section{Zhang 2011}

Zhang D, Freemantle N, Cheng KK. Are randomized trials conducted in China or India biased? A comparative empirical analysis. Journal of Clinical Epidemiology 2011;64(1):90-5. [PUBMED: 20554429]

\section{References to other published versions of this review Matthews 2012 \\ Matthews P, Horder J. Selective noradrenaline reuptake inhibitors for schizophrenia. Cochrane Database of Systematic Reviews 2012, Issue 11. [DOI: 10.1002/14651858.CD010219]}

\section{Ball 2011}

$\begin{array}{ll}\text { Methods } & \text { Allocation: randomised } \\ \text { Blindness: double blind, parallel groups } \\ \text { Duration: } 24 \text { weeks }\end{array}$

Darticipants
$\mathrm{N}=37$ (data from 36)
Age: 18 to 65 years (mean 47 years)
Sex: $11 \mathrm{~F}, 25 \mathrm{M}$
History: clinically stable outpatients, mean baseline BPRS total score 33 (mild severity), duration of ill-
ness not reported but all patients treated for at least 6 months, participants from Maryland Psychiatric
Research Center Outpatient Program, DSM-IV diagnoses, taking clozapine ( $\mathrm{N}=18$ with 10 also taking
risperidone) or olanzapine ( $\mathrm{N}=18$ ) for at least 6 months with weight gain greater than $7 \%$ since initia-
tion
Exclusions: those on medication with central noradrenergic effects or associated with weight gain (un-
less stable for over 6 months), other weight-loss medication, pregnancy, DSM-IV mental retardation or
alcohol or substance dependence in 6 months or abuse in the last 1 month (excluding nicotine), uncon-
trolled hypertension


Ball 2011 (Continued)

Outcomes
Mental state: BPRS total score†§

Cognitive functioning: composite neuropsychological z-score $§$

Leaving the study early

Adverse effects: specific side-effects (tremor, QTc prolongation)§, weight change§

Unable to use (no means or SD reported)

Mental state: BPRS subscales, SANS

Clinical global response: CGI

Cognitive functioning: Individual neuropsychological tests

Adverse effects: AIMS, SAS, SEC
Given a weight loss programme (Weight Watchers) in both arms of the trial using a token system to encourage compliance

Patient population overlaps with Kelly 2009 but each study randomised separately

Funded by Eli Lilly and $\mathrm{NIH}$, no declarations of interest

Additional correspondence with author M Patricia Ball (PB) clarifying randomisation and blinding (see below)

Date study conducted not reported but overlapping study Kelly 2009 conducted 2004 to 2006 in the United States

\section{Risk of bias}

Bias Authors' judgement Support for judgement

Random sequence genera- Low risk tion (selection bias)

\section{Computer generated randomisation}

"Randomization was conducted separately within those 3 strata, with an additional stratification by site (MPRC inpatient, MPRC outpatient or VA) within each of the 3 trial participation strata, using a randomizated block design[ $\left.{ }^{\star}\right]$

"A permuted block design was used to assign treatments within the three strata described in my previous e-mail. The random treatment assignments sequences were generated in advance by the study statistician, and prior to the completion of the study the only persons with access to the sequence were the study statistician and the unblinded pharmacist who prepared the study medication (identical appearing capsules containing atomoxetine or placebo) for the patients. When a treatment assignment was requested for a new participant, the statistician e-mailed a code number to the pharmacist, who kept a list of the code numbers and corresponding treatments in a locked cabinet in the his/her office" PB

*The 3 strata are those participating in Ball 2011, Kelly 2009, or both

\begin{tabular}{lll}
\hline $\begin{array}{l}\text { Allocation concealment } \\
\text { (selection bias) }\end{array}$ & Low risk & As above \\
\hline $\begin{array}{l}\text { Blinding of participants } \\
\text { and personnel (perfor- } \\
\text { mance bias) }\end{array}$ & Low risk & As above \\
All outcomes & & \\
\hline
\end{tabular}


Ball 2011 (Continued)

Blinding of outcome as-
sessment (detection bias)

All outcomes

\begin{tabular}{|c|c|c|}
\hline $\begin{array}{l}\text { Incomplete outcome data } \\
\text { (attrition bias) }\end{array}$ & High risk & $\begin{array}{l}\text { Withdrawals accounted for but evidence of differences in reasons between } \\
\text { treatment and placebo arms }\end{array}$ \\
\hline
\end{tabular}

All outcomes

Selective reporting (re- High risk $\quad$ Selective reporting of outcome measures evident
porting bias)

\begin{tabular}{ll}
\hline Other bias $\quad$ Low risk $\quad$ No other specific biases noted \\
\hline
\end{tabular}

\section{Eli Lilly 2006}

$\begin{array}{ll}\text { Methods } & \text { Allocation: randomised } \\ \text { Blindness: double blind, parallel groups } & \\ \text { Duration: } 8 \text { weeks }\end{array}$

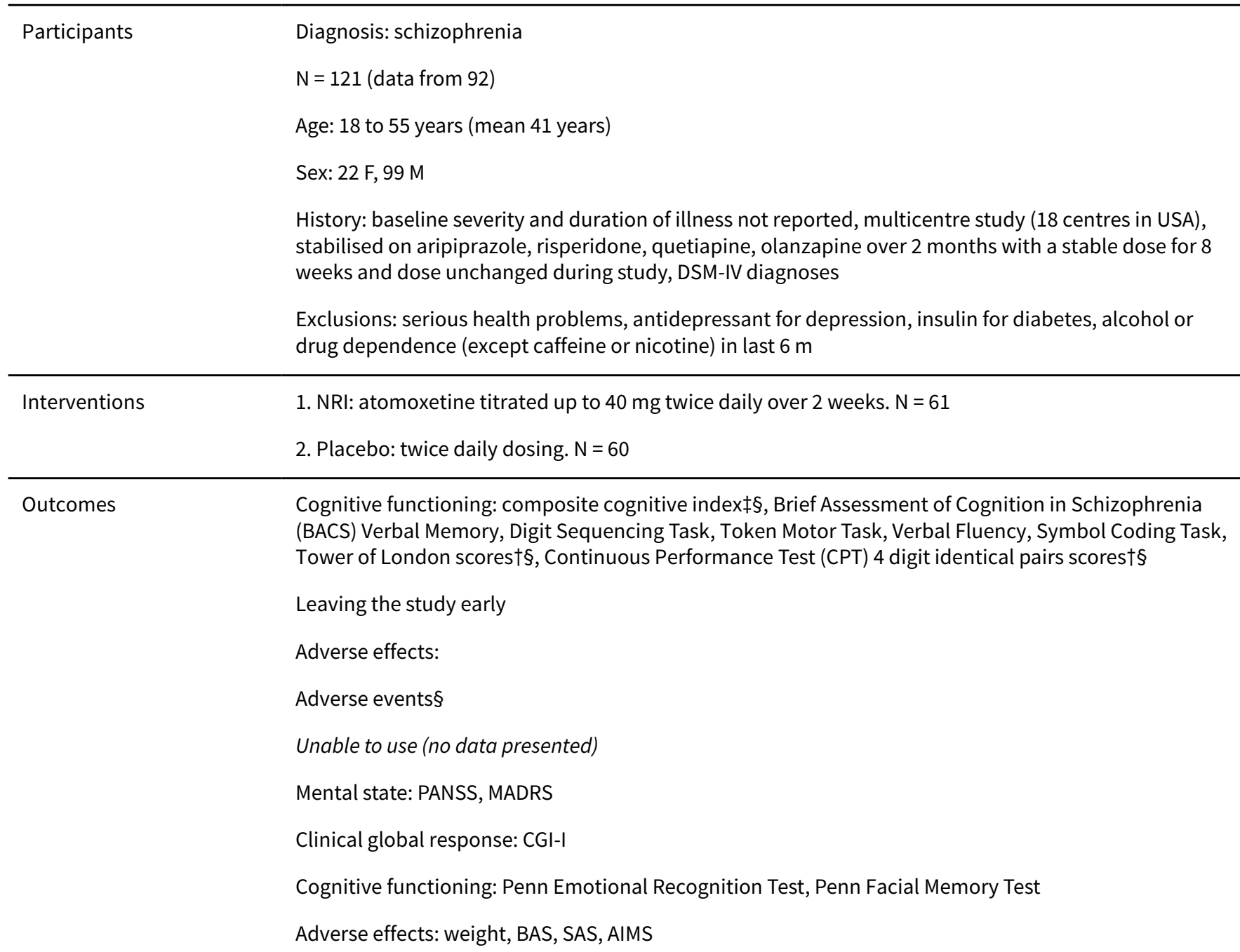


Eli Lilly 2006 (Continued)

Social or general functioning: Drug Attitude Inventory (DAI-10), Social Functioning Scale, Independent Living Scale

\section{Notes}

ITT LOCF for all patients with at least 1 post-baseline observation

Data provided by Eli Lilly

Funded by Eli Lilly, no declarations of interest

Study conducted from 2003 to 2005 in the United States

\section{Risk of bias}

\begin{tabular}{|c|c|c|}
\hline Bias & Authors' judgement & Support for judgement \\
\hline $\begin{array}{l}\text { Random sequence genera- } \\
\text { tion (selection bias) }\end{array}$ & Unclear risk & Method not described \\
\hline $\begin{array}{l}\text { Allocation concealment } \\
\text { (selection bias) }\end{array}$ & Unclear risk & Method not described \\
\hline $\begin{array}{l}\text { Blinding of participants } \\
\text { and personnel (perfor- } \\
\text { mance bias) } \\
\text { All outcomes }\end{array}$ & Unclear risk & Method not described \\
\hline $\begin{array}{l}\text { Blinding of outcome as- } \\
\text { sessment (detection bias) } \\
\text { All outcomes }\end{array}$ & Unclear risk & Method not described \\
\hline $\begin{array}{l}\text { Incomplete outcome data } \\
\text { (attrition bias) } \\
\text { All outcomes }\end{array}$ & High risk & $\begin{array}{l}\text { More withdrawals in the atomoxetine arm ( } 6 \text { vs } 2 \text { ) with reasons for withdrawals } \\
\text { in the atomoxetine arm frequently related to psychiatric symptoms (e.g. hos- } \\
\text { pitalisation for acute psychosis) }\end{array}$ \\
\hline $\begin{array}{l}\text { Selective reporting (re- } \\
\text { porting bias) }\end{array}$ & High risk & Selective reporting of outcome measures evident \\
\hline Other bias & Unclear risk & $\begin{array}{l}\text { Baseline imbalance in cognitive scores but not statistically significant and un- } \\
\text { clear if this has had a significant impact on the results }\end{array}$ \\
\hline
\end{tabular}

\section{Friedman 2008}

\begin{tabular}{ll}
\hline Methods & Allocation: randomised \\
Blindness: double blind, parallel groups \\
Duration: 8 weeks \\
\hline
\end{tabular}

Diagnosis: schizophrenia
Participants 20 (15 completers)
Age: not reported
Sex: not reported
History: baseline severity borderline ill (mean PANSS 66), duration of illness not reported, psychiatry
outpatients in the New York area, DSM-IV diagnoses, taking risperidone, olanzapine, quetiapine, or
aripiprazole on stable dose for $\geq 4$ weeks, no other psychotropics


Friedman 2008 (Continued)

\section{Exclusions: not reported}

Interventions $\quad$ 1. NRI: atomoxetine $40 \mathrm{mg}$ once daily for 4 weeks then $40 \mathrm{mg}$ twice daily for 4 weeks. $\mathrm{N}=7$

2. Placebo: once daily for 4 weeks then twice daily for 4 weeks. $N=8$

Outcomes

Mental state: PANSS positive, negative, and general scores $† \S$

Cognitive functioning: Brief Assessment of Cognition in Schizophrenia (BACS) composite score, list learning test, digit sequencing, token motor task, category instances test, controlled oral word association test, Tower of London test, symbol coding change z-scores $†$

Leaving the study early

Social or general functioning: Specific Level of Functioning (SLOF) scale physical functioning, personal care skills, interpersonal relationships, social acceptability, activities, work skills change scores $† \S$

Unable to use (data not reported)

Cognitive functioning: N-back task
FMRI study of N-back task

LOCF and completer data reported

Funding from Eli Lilly, NIH, US Dept Veterans Affairs. Reported conflicts of interest include shares, grants, and advisory boards for Eli Lilly

Study conducted in the United States but the date was not reported

\section{Risk of bias}

\begin{tabular}{|c|c|c|}
\hline Bias & Authors' judgement & Support for judgement \\
\hline $\begin{array}{l}\text { Random sequence genera- } \\
\text { tion (selection bias) }\end{array}$ & Unclear risk & Method not described \\
\hline $\begin{array}{l}\text { Allocation concealment } \\
\text { (selection bias) }\end{array}$ & Unclear risk & Method not described \\
\hline $\begin{array}{l}\text { Blinding of participants } \\
\text { and personnel (perfor- } \\
\text { mance bias) } \\
\text { All outcomes }\end{array}$ & Unclear risk & $\begin{array}{l}\text { "double-blind...matching placebo" } \\
\text { Non-concurrence in ratings (PM: unclear risk; MP: low risk) and overall unclear } \\
\text { risk agreed after discussion }\end{array}$ \\
\hline $\begin{array}{l}\text { Blinding of outcome as- } \\
\text { sessment (detection bias) } \\
\text { All outcomes }\end{array}$ & Unclear risk & Method not described \\
\hline $\begin{array}{l}\text { Incomplete outcome data } \\
\text { (attrition bias) } \\
\text { All outcomes }\end{array}$ & Low risk & $\begin{array}{l}\text { Similar number of withdrawals between groups, reasons for withdrawals dis- } \\
\text { similar between groups but overall unlikely to impact on effect sizes }\end{array}$ \\
\hline $\begin{array}{l}\text { Selective reporting (re- } \\
\text { porting bias) }\end{array}$ & Low risk & All outcomes of interest reported \\
\hline Other bias & Low risk & No other specific biases noted \\
\hline
\end{tabular}


Ganguli 2008

\begin{tabular}{ll}
\hline Methods & Allocation: randomised \\
Blindness: double blind, parallel groups \\
Duration: 26 weeks
\end{tabular}

\begin{tabular}{|c|c|c|}
\hline \multirow[t]{7}{*}{ Participants } & \multicolumn{2}{|c|}{ Diagnosis: schizophrenia or schizoaffective disorder } \\
\hline & \multicolumn{2}{|l|}{$\mathrm{N}=60$ (30 completers) } \\
\hline & \multicolumn{2}{|l|}{ Age: 18 to 65 years } \\
\hline & \multicolumn{2}{|l|}{ Sex: not reported } \\
\hline & \multicolumn{2}{|c|}{$\begin{array}{l}\text { History: baseline severity mild illness (PANSS } \leq 80 \text { ), duration of illness not reported, prominent negative } \\
\text { symptoms, } 30 \text { days stable dose of antipsychotics (and other psychotropics), no hospital admission in } \\
\text { last } 30 \text { days, DSM diagnosis of schizophrenia, birth control, } \geq 10 \text { on } 5 \text { SANS global ratings, } \geq 3 \text { on } 2 \text { glob- } \\
\text { al items and mean score } \leq 4 \text { on General Life Satisfaction on Lehman's QoL }\end{array}$} \\
\hline & \multicolumn{2}{|c|}{$\begin{array}{l}\text { Exclusions: inability to give informed consent, alcohol or substance misuse or dependence in last } 6 \\
\text { months, narrow angle glaucoma or organic brain disease, history of uncontrolled diabetes mellitus or } \\
\text { hypertension, liver disease, cerebrovascular disease or myocardial infarction in last } 3 \text { months, use of } \\
\text { venlafaxine, MAOI, cytochrome p450 }\end{array}$} \\
\hline & \multicolumn{2}{|c|}{$2 \mathrm{~d} 6$ inhibitors (e.g. fluoxetine), salbutamol, or psychostimulant currently or last 2 weeks } \\
\hline \multirow[t]{2}{*}{ Interventions } & \multicolumn{2}{|c|}{ 1. NRI: atomoxetine $40 \mathrm{mg}$ twice daily } \\
\hline & \multicolumn{2}{|l|}{ 2. Placebo } \\
\hline \multirow[t]{6}{*}{ Outcomes } & \multicolumn{2}{|c|}{ Unable to use (data not reported) } \\
\hline & \multicolumn{2}{|c|}{ Mental state: SANS, PANSS, Calgary Depression Scale } \\
\hline & \multicolumn{2}{|c|}{ Clinical global response: CGI } \\
\hline & \multicolumn{2}{|c|}{ Cognitive functioning: Cognitive battery } \\
\hline & \multicolumn{2}{|c|}{ Quality of life: Lehman's quality of life scale (QoL) } \\
\hline & \multicolumn{2}{|c|}{ Social or general functioning: General Assessment of Functioning (GAF) } \\
\hline \multirow[t]{2}{*}{ Notes } & \multicolumn{2}{|c|}{ Funded by the Stanley Research Foundation, conflicts of interest not reported } \\
\hline & \multicolumn{2}{|c|}{ Date conducted not reported } \\
\hline \multicolumn{3}{|l|}{ Risk of bias } \\
\hline Bias & Authors' judgement & Support for judgement \\
\hline \multirow{2}{*}{$\begin{array}{l}\text { Random sequence genera- } \\
\text { tion (selection bias) }\end{array}$} & \multirow[t]{2}{*}{ Unclear risk } & "randomised double blind" \\
\hline & & Method not described \\
\hline $\begin{array}{l}\text { Allocation concealment } \\
\text { (selection bias) }\end{array}$ & Unclear risk & Method not described \\
\hline $\begin{array}{l}\text { Blinding of participants } \\
\text { and personnel (perfor- } \\
\text { mance bias) } \\
\text { All outcomes }\end{array}$ & Unclear risk & Method not described \\
\hline
\end{tabular}


Ganguli 2008 (Continued)

Blinding of outcome as- Unclear risk Method not described sessment (detection bias)

All outcomes

\begin{tabular}{lll}
$\begin{array}{l}\text { Incomplete outcome data } \\
\text { (attrition bias) } \\
\text { All outcomes }\end{array}$ & High risk & Half of all participants did not complete the trial \\
\hline $\begin{array}{l}\text { Selective reporting (re- } \\
\text { porting bias) }\end{array}$ & High risk & No useable outcomes reported \\
\hline Other bias & Unclear risk & $\begin{array}{l}\text { Due to limited published information about this trial it was not possible to es- } \\
\text { tablish other sources of bias }\end{array}$ \\
\hline
\end{tabular}

Hinkelmann 2013

\begin{tabular}{ll}
\hline Methods & Allocation: randomised \\
Blindness: double blind, parallel groups \\
Duration: 4 weeks
\end{tabular}

Diagnosis: schizophrenia
N $=51$ (43 completers)
Age: Mean 40 years
Sex: $17 \mathrm{~F}, 34 \mathrm{M}$
History: baseline severity markedly ill (CGI 5), mean duration of illness 12 years, DSM-IV, $\geq 4$ on at least
1 PANSS negative item, 2 weeks stable antipsychotic dose (olanzapine 5, quetiapine 5, clozapine 7,
amisulpride 9 , risperidone 13, aripiprazole 2, combined 10) kept stable throughout study
Exclusions: alcohol or substance abuse, other psychiatric or somatic disorders, abnormal laboratory
findings

Interventions

1. NRI: reboxetine $4 \mathrm{mg}$ capsule once daily and increased as needed to twice daily after 1 week. $\mathrm{N}=19$

2. Placebo: 1 capsule once daily and increased as needed to twice daily after 1 week. $\mathrm{N}=16$

3. Active control: citalopram $20 \mathrm{mg}$ capsule once daily and increased as needed to twice daily after 1 week. $\mathrm{N}=16$

Outcomes

Mental state: PANSS positive, negative, general, HRSD scorest§

Clinical global response: $\mathrm{CGI}-\mathrm{S} † \S$

Leaving the study early

Adverse effects: 'Serious' side-effects§, agitation side-effect§

Unable to use (data not reported)

Mental state: PANSS total, PANSS negative responder rate

Adverse effects: UKU side-effects rating scale, AIMS, SAS, BAS, other specific side-effects 
ITT LOCF analysis for those with data after 1 week

PANSS 'global' results reported but these appear to be the PANSS general subscale and are treated as such

Funding from the Stanley Medical Research Institute, reported conflicts of interest include multiple honoraria, expenses, and grants from various pharmaceutical companies including Eli Lilly and Pfizer

Study conducted from 2002 to 2008, although it is not explicitly stated it is presumed to have been carried out in Germany

\section{Risk of bias}

\begin{tabular}{|c|c|c|}
\hline Bias & Authors' judgement & Support for judgement \\
\hline $\begin{array}{l}\text { Random sequence genera- } \\
\text { tion (selection bias) }\end{array}$ & Low risk & $\begin{array}{l}\text { "The randomization was organized using the PLAN procedure from the SAS/ } \\
\text { STAT software" }\end{array}$ \\
\hline $\begin{array}{l}\text { Allocation concealment } \\
\text { (selection bias) }\end{array}$ & Low risk & $\begin{array}{l}\text { "Allocation codes for each patient were provided in sealed envelopes through } \\
\text { the pharmacy" }\end{array}$ \\
\hline $\begin{array}{l}\text { Blinding of participants } \\
\text { and personnel (perfor- } \\
\text { mance bias) } \\
\text { All outcomes }\end{array}$ & Unclear risk & Method not described \\
\hline $\begin{array}{l}\text { Blinding of outcome as- } \\
\text { sessment (detection bias) } \\
\text { All outcomes }\end{array}$ & Unclear risk & Method not described \\
\hline $\begin{array}{l}\text { Incomplete outcome data } \\
\text { (attrition bias) } \\
\text { All outcomes }\end{array}$ & Unclear risk & $\begin{array}{l}\text { More withdrawals in the reboxetine arm ( } 3 \text { vs } 1 \text { ) and reasons for withdrawals } \\
\text { not given }\end{array}$ \\
\hline $\begin{array}{l}\text { Selective reporting (re- } \\
\text { porting bias) }\end{array}$ & Unclear risk & $\begin{array}{l}\text { Evidence of some secondary outcome measures not reported from Stanley } \\
\text { Foundation grant abstract }\end{array}$ \\
\hline Other bias & Low risk & $\begin{array}{l}\text { Some baseline imbalance but not statistically significant and unlikely to have } \\
\text { a major impact }\end{array}$ \\
\hline
\end{tabular}

\section{Kelly 2009}

\begin{tabular}{ll}
\hline Methods & Allocation: randomised \\
Blindness: double blind, parallel groups \\
Duration: 8 weeks \\
\hline
\end{tabular}

\section{Participants}

Diagnosis: schizophrenia or schizoaffective disorder

$\mathrm{N}=32$ (data from 22)

Age: 18 to 60 years (mean 49 years)

\section{Sex: $6 \mathrm{~F}, 16 \mathrm{M}$}

History: baseline severity markedly ill (CGI 4.5), mean duration of illness not reported directly but mean 24 years since first hospitalisation, inpatients and outpatients, DSM-IV, cognitive impairment (RBANS $\leq$ 90), 4-week stable dose of atypical antipsychotics (olanzapine 13, quetiapine 2, risperidone 11), 2 week 
Kelly 2009 (Continued)

stabilisation phase before randomisation, psychotropics at a stable dose for 4 weeks, no medication changes during study

Exclusions: on clozapine or aripiprazole, organic brain disease, alcohol or substance abuse in last 1 month or dependence in last 6 months, pregnancy, uncontrolled hypertension, venlafaxine or MAOI use, anticholinergics or benzodiazepines (but see below)

Interventions

1. NRI: atomoxetine $40 \mathrm{mg}$ capsule once daily for 2 weeks then $80 \mathrm{mg}$ once daily for 6 weeks. $\mathrm{N}=16$

2. Placebo: 1 capsule once daily for 2 weeks then 2 capsules once daily for 6 weeks. $N=16$

Outcomes

Mental state: BPRS total, positive, anxiety/depression, and hostility scores, modified SANS total score†§

Clinical global response: CGI†§

Cognitive functioning: Composite mean scores for cognitive testsł, WAIS-III Letter-Number Sequencing and Number-Sequencing Test, WAIS-III Digit Symbol, Grooved Pegboard, Letter Fluency, Woodcock Johnson Planning, California Verbal Learning Test, Brief Visuospatial Memory Test, distractibility version of the GDS Continuous Performance Test (many scores considered to be skewed) †§

Leaving the study early

Adverse effects: SAS, AIMS, BAS $\geq 2^{\star} \dagger \S$, side-effects checklist§, weight§

Notes

Allowed benztropine or lorazepam for anxiety, agitation, or akathisia

2 patients taking aripiprazole excluded for taking aripiprazole after randomisation

Patient population overlaps with Ball 2011 but each study randomised separately

Funding from NIH, US Dept Veterans Affairs, Stanley Medical Research Institute. Eli Lilly provided study medication. Reported conflicts of interest include multiple honoraria, grants, and advisory boards for multiple pharmaceutical companies including Pfizer and Eli Lilly

Study conducted from 2004 to 2006 in the United States

\section{Risk of bias}

\begin{tabular}{|c|c|c|}
\hline Bias & Authors' judgement & Support for judgement \\
\hline $\begin{array}{l}\text { Random sequence genera- } \\
\text { tion (selection bias) }\end{array}$ & Low risk & "permuted block randomization system" \\
\hline $\begin{array}{l}\text { Allocation concealment } \\
\text { (selection bias) }\end{array}$ & Low risk & Method not described but likely same as for Ball 2011 \\
\hline $\begin{array}{l}\text { Blinding of participants } \\
\text { and personnel (perfor- } \\
\text { mance bias) } \\
\text { All outcomes }\end{array}$ & Low risk & $\begin{array}{l}\text { "The central unblinded pharmacist will be notified of the treatment assign- } \\
\text { ment, and will inform unblinded pharmacists at the other sites about which } \\
\text { study medication to dispense } \\
\text { "All raters, investigators and other staff will be blind to treatment assignment } \\
\text { except for the pharmacist" }\end{array}$ \\
\hline $\begin{array}{l}\text { Blinding of outcome as- } \\
\text { sessment (detection bias) } \\
\text { All outcomes }\end{array}$ & Unclear risk & Method not described \\
\hline $\begin{array}{l}\text { Incomplete outcome data } \\
\text { (attrition bias) } \\
\text { All outcomes }\end{array}$ & Unclear risk & $\begin{array}{l}\text { Withdrawals accounted for but evidence of slight differences in withdrawal } \\
\text { reasons between treatment and placebo arms with } 2 \text { in placebo arm showing } \\
\text { worsening psychosis and } 1 \text { in atomoxetine arm 'feeling strange' although un- } \\
\text { clear if this could significantly influence the results }\end{array}$ \\
\hline
\end{tabular}


Kelly 2009 (Continued)

Selective reporting (re- Low risk $\quad$ All outcomes of interest reported
porting bias)

\begin{tabular}{ll}
\hline Other bias $\quad$ High risk & $\begin{array}{l}2 \text { patients taking aripiprazole excluded from the atomoxetine arm after ran- } \\
\text { domisation on the grounds that aripiprazole is an exclusion criterion (although } \\
\text { it is not listed as such in the protocol) }\end{array}$
\end{tabular}

\section{Kurland 1981}

\begin{tabular}{ll}
\hline Methods & Allocation: randomised \\
Blindness: double blind, parallel groups \\
Duration: 4 weeks
\end{tabular}

Participants
$\mathrm{N}=28$ (22 completers)
Age: 19 to 53 years
Sex: not reported
History: baseline severity or duration of illness not reported, HRSD $\geq 18$, stabilised for $\geq 2$ weeks on con
stant dose of chlorpromazine or haloperidol
Exclusions: pregnancy, significant physical illness, epilepsy, mental retardation, senility, organic brain
disease, history of alcohol or drug abuse, investigational drugs in the last 2 weeks, sensitivity to test
compounds, MAOI in last 2 weeks, treatment with antiparkinsonian medication

Interventions

1. NRI: viloxazine $50 \mathrm{mg}$ tablets 3 times per day in week 1 and increased by 1 tablet daily each week until a reduction $\geq 40 \%$ on HRSD (if HRSD subsequently increased the titration schedule was continued) up to a maximum of $300 \mathrm{mg}$ daily. $\mathrm{N}=10$

2. Placebo: tablets titrated as above. $\mathrm{N}=12$

\begin{tabular}{|c|c|}
\hline \multirow[t]{5}{*}{ Outcomes } & Mental state/clinical global response: Improvement§ \\
\hline & Leaving the study early \\
\hline & Unable to use (data not reported) \\
\hline & Mental state: BPRS, HRSD, Zung Self Rating Depression Scale \\
\hline & Clinical global response: CGI \\
\hline \multirow[t]{2}{*}{ Notes } & $\begin{array}{l}\text { Funding not reported but authors acknowledge assistance from } \mathrm{ICl} \text {, declarations of interest not report- } \\
\text { ed }\end{array}$ \\
\hline & $\begin{array}{l}\text { Date study conducted not reported; although it is not explicitly stated it is presumed to have been car- } \\
\text { ried out in the United States }\end{array}$ \\
\hline
\end{tabular}

\section{Risk of bias}

\begin{tabular}{lll}
\hline Bias & Authors' judgement & Support for judgement \\
\hline $\begin{array}{l}\text { Random sequence genera- } \\
\text { tion (selection bias) }\end{array}$ & Unclear risk & Method not described \\
\hline
\end{tabular}


Kurland 1981 (Continued)

\begin{tabular}{|c|c|c|}
\hline $\begin{array}{l}\text { Allocation concealment } \\
\text { (selection bias) }\end{array}$ & Unclear risk & Method not described \\
\hline $\begin{array}{l}\text { Blinding of participants } \\
\text { and personnel (perfor- } \\
\text { mance bias) } \\
\text { All outcomes }\end{array}$ & Unclear risk & $\begin{array}{l}\text { "double-blind...identical-appearing placebo tablets" } \\
\text { Non-concurrence in ratings (PM: unclear risk; MP: low risk) and overall unclear } \\
\text { risk agreed after discussion }\end{array}$ \\
\hline
\end{tabular}

All outcomes

Unclear risk

Method not described in detail. "During the course of the study each patient

Blinding of outcome aswas interviewed and evaluated by the same investigator"

All outcomes

\begin{tabular}{lll}
\hline $\begin{array}{l}\text { Incomplete outcome data } \\
\text { (attrition bias) } \\
\text { All outcomes }\end{array}$ & Low risk & Withdrawals equal across arms and reasons unlikely to be related to treatment \\
\hline $\begin{array}{l}\text { Selective reporting (re- } \\
\text { porting bias) }\end{array}$ & High risk & Most outcomes not reported in any detail \\
\hline Other bias & Unclear risk & $\begin{array}{l}\text { Some methodological flaws (e.g. protocol includes excluding patients with } \\
\text { side-effects in certain situations) but it was not clear that this had an effect on } \\
\text { the results }\end{array}$ \\
\hline
\end{tabular}

\section{Li 2008}

$\begin{array}{ll}\text { Methods } & \text { Allocation: randomised } \\ \text { Blindness: double blind, parallel groups } & \\ \text { Duration: } 12 \text { weeks }\end{array}$

Diagnosis: schizophrenia
Participants 118 (114 completers)
Age: 18 to 65 years (mean 50 years)
Sex: All male
History: baseline severity mild (mean PANSS 75), mean duration of illness 27 years ( $>3$ years), inpa-
tients in Futuijunren Hospital, Hebei Province, with chronic schizophrenia by Chinese Classification of
Mental Disorders (3rd Ed), predominantly negative symptoms (by criteria of Andreasen 1982$)$, clozapine
at stable dose for $\geq 3$ months, PANSS negative $\geq 30$, PANSS total $\geq 60$, no other psychotropics, duration
of illness: $>3$ years (mean 27 years)
Exclusions: post-schizophrenic depression, schizoaffective disorder, abnormal physical examination or
blood tests, significant physical illness, epilepsy, organic brain disease, alcohol or drug abuse

Interventions 1 NRI: reboxetine $4 \mathrm{mg}$ daily increased to $8 \mathrm{mg}$ daily after the third day. $\mathrm{N}=57$

2. Placebo. $\mathrm{N}=57$

Outcomes Mental state: PANSS total, positive, negative, and general scores ${ }^{\prime}$

Quality of life: General Quality Of Life Inventory-74 (GQOLI-74) total and subscales (a published quality of life scale adapted for the Chinese population) $\dagger$

Adverse effects: Treatment Emergent Symptoms Scale† 
Social or general functioning: Scale of Social-skills for Psychiatric Inpatients (SSPI) subscales (a published Chinese scale for social functioning) $\dagger$

Unable to use (data not reported)

Adverse effects: leaving the study early not reported by treatment arm (1 seizure and 3 discharged from hospital),

weight

Translated from Chinese
Patients could receive benzodiazepines or Z-drugs for insomnia.
Source of funding and conflicts of interest not reported
Study conducted in 2006 in China
Reported SDs for continuous outcomes appear significantly smaller than for other trials. Although the
paper does not explicitly report that these are SDs rather than SEs (stating they are " $\bar{x} \pm s ")$ examination
of the reported t-tests confirms that these are SDs

\section{Risk of bias}

\begin{tabular}{lll}
\hline Bias & Authors' judgement & Support for judgement \\
\hline $\begin{array}{l}\text { Random sequence genera- } \\
\text { tion (selection bias) }\end{array}$ & Unclear risk & Method not described \\
\hline $\begin{array}{l}\text { Allocation concealment } \\
\text { (selection bias) }\end{array}$ & Unclear risk & $\begin{array}{l}\text { Method not described in detail, "unblinding by pharmacy at the end of the } \\
\text { study" }\end{array}$ \\
\hline $\begin{array}{l}\text { Blinding of participants } \\
\text { and personnel (perfor- } \\
\text { mance bias) } \\
\text { All outcomes }\end{array}$ & Low risk & "unblinding by pharmacy at the end of the study" \\
\hline $\begin{array}{l}\text { Blinding of outcome as- } \\
\text { sessment (detection bias) }\end{array}$ & Unclear risk & $\begin{array}{l}\text { "the reboxetine and placebo (starch powder) were placed in identical looking } \\
\text { capsules" }\end{array}$ \\
\hline $\begin{array}{l}\text { Incomplete outcome data } \\
\text { (attrition bias) }\end{array}$ & Low risk & Method not described \\
$\begin{array}{l}\text { All outcomes } \\
\begin{array}{l}\text { Selective reporting (re- } \\
\text { porting bias) }\end{array}\end{array}$ & Unclear risk & $\begin{array}{l}\text { Withdrawals not reported separately for treatment arms but unlikely to have a } \\
\text { significant impact on outcomes }\end{array}$ \\
\hline \begin{tabular}{l} 
Other bias \\
\hline
\end{tabular} & Low risk & Some outcomes of interest not reported \\
\hline
\end{tabular}

\section{Poyurovsky 2003}

$\begin{array}{ll}\text { Methods } & \text { Allocation: randomised } \\ \text { Blindness: double blind, parallel groups } \\ \text { Duration: } 6 \text { weeks }\end{array}$

Duration: 6 weeks

Participants Diagnosis: schizophrenia


Poyurovsky 2003 (Continued)

$$
N=26
$$

Age: mean 31 years

Sex: 9 F, $17 \mathrm{M}$

History: baseline severity moderately severe (mean CGI 4.7), mean duration of illness 6 years, hospitalised first episode schizophrenia in the Tirat Carmel Mental Health Center, DSM-IV, < 4 weeks antipsychotic exposure in last 6 months, no previous olanzapine and current recommendation for olanzapine, given $10 \mathrm{mg}$ olanzapine daily for 6 weeks

Exclusions: uncooperative, aggressive or suicidal behaviour, diabetes mellitus, obesity

\begin{tabular}{|c|c|}
\hline Interventions & $\begin{array}{l}\text { 1. NRI: reboxetine } 4 \mathrm{mg} \text { daily. } \mathrm{N}=13 \\
\text { 2. Placebo. } \mathrm{N}=13\end{array}$ \\
\hline Outcomes & $\begin{array}{l}\text { Mental state: Scale for the Assessment of Positive Symptoms (SAPS), SANS, HRSD†¥ } \\
\text { Clinical global response: CGI†¥ } \\
\text { Leaving the study early } \\
\text { Adverse effects: Weight, gaining } \geq 7 \% \text { of initial weight } ¥ \\
\text { Unable to use (means and SD not reported) } \\
\text { Adverse effects: BAS, SAS }\end{array}$ \\
\hline Notes & $\begin{array}{l}\text { Poyurovsky } 2003 \text { reports a reboxetine dose of } 2 \mathrm{mg} \text { twice daily while Amrami-Weizman } 2013 \text { reports } 4 \\
\text { mg twice daily } \\
\text { Trihexyphenidyl ( } 5 \text { to } 10 \mathrm{mg} \text { daily) and lorazepam ( } 1 \text { to } 3 \mathrm{mg} \text { daily) allowed as needed but no other psy- } \\
\text { chotropics } \\
\text { Funding not reported but Agis Industries Ltd. (Israel) provided medication, conflicts of interest not re- } \\
\text { ported } \\
\text { Study conducted in Israel but the date conducted not reported }\end{array}$ \\
\hline
\end{tabular}

\section{Risk of bias}

\begin{tabular}{lll}
\hline Bias & Authors' judgement & Support for judgement \\
\hline $\begin{array}{l}\text { Random sequence genera- } \\
\text { tion (selection bias) }\end{array}$ & Low risk & $\begin{array}{l}\text { "...participants were allocated according to entries of a table of random num- } \\
\text { bers" }\end{array}$ \\
\hline $\begin{array}{l}\text { Allocation concealment } \\
\text { (selection bias) }\end{array}$ & Unclear risk & Method not described \\
\hline $\begin{array}{l}\text { Blinding of participants } \\
\begin{array}{l}\text { and personnel (perfor- } \\
\text { mance bias) } \\
\text { All outcomes }\end{array}\end{array}$ & Unclear risk & Method not described \\
\hline
\end{tabular}

\begin{tabular}{lll}
$\begin{array}{l}\text { Blinding of outcome as- } \\
\begin{array}{l}\text { sessment (detection bias) } \\
\text { All outcomes }\end{array}\end{array}$ & Low risk & $\begin{array}{l}\text { "All weight measurements were performed by a research nurse who was blind } \\
\text { to the patients' treatment assignment... }\end{array}$ \\
& $\begin{array}{l}\text { Clinical ratings were completed at baseline and at week } 6 \text { by the same trained } \\
\text { psychiatrist... who was blind to the patients' treatment assignments" }\end{array}$ \\
\hline
\end{tabular}


Poyurovsky 2003 (Continued)

Incomplete outcome data Low risk Equal withdrawals and reasons equally distributed (attrition bias)

All outcomes

Selective reporting (re- Low risk All outcomes of interest reported

porting bias)

Other bias Low risk No other specific biases noted

Poyurovsky 2007

$\begin{array}{ll}\text { Methods } & \text { Allocation: randomised } \\ \text { Blindness: double blind, parallel groups } \\ \text { Duration: } 6 \text { weeks }\end{array}$

Participants Diagnosis: schizophrenia or schizophreniform disorder

$N=59$

Age: 19 to 48 years (mean 30 years)

Sex: $21 \mathrm{~F}, 38 \mathrm{M}$

History: baseline severity moderately ill (mean CGI 4.2), mean duration of illness 3.5 years, inpatients with first episode of psychosis in Tirat Carmel Mental Health Center, DSM-IV by SCID-I, under 4 weeks antipsychotic treatment, recommendation for olanzapine (given $10 \mathrm{mg}$ daily olanzapine during study), no other antipsychotics, antidepressants, or mood stabilisers were permitted

Exclusions: organic brain damage, alcohol or drug abuse, other Axis-I psychiatric disorders including major mood disorders, aggressive or suicidal behaviour, medical illnesses that could affect body weight (e.g. diabetes mellitus and hypothyroidism), obesity

Interventions

1. NRI: reboxetine $2 \mathrm{mg}$ twice daily. $\mathrm{N}=31$

2. Placebo: twice daily. $\mathrm{N}=28$

Outcomes $\quad$ Mental state: SAPS, SANS, HRSD†¥

Clinical global response: CGI†¥

Cognitive functioning: Automated Neuropsychological Assessment Metrics (ANAM) computerised battery percentage correct and reaction time for correct responses, simple reaction time, code substitution, code substitution immediate recall, mental rotation task, matching to sample test, Continuous Performance Test, code substitution delayed recall†¥, Wisconsin Card Sorting Test categories completed and perseverative errorsł¥

Leaving the study early

Adverse effects: BAS, SAS $¥ ¥$, use of 'as needed' medication, weight, gaining $\geq 7 \%$ of initial weight $¥$

Notes

Administration of trihexyphenidyl ( $5 \mathrm{mg} /$ day) or biperiden ( 2 to $4 \mathrm{mg} /$ day) for extrapyrmidal side-effects and lorazepam (1 to $3 \mathrm{mg} /$ day) or diazepam ( $5 \mathrm{mg} /$ day) for insomnia or agitation were allowed as needed

Intention-to-treat analysis with imputation by regression for missing weights 
Poyurovsky 2007 (Continued)

The exact dosing regime of reboxetine is unclear as it is reported variously as $2 \mathrm{mg}$ twice daily (Poyurovsky et al 2007), 4 mg once daily (Poyurovsky et al 2009), and 4 mg twice daily (Amrami-Weizman 2013).

In Poyurovsky et al 2007 under 4 weeks of antipsychotic treatment is reported but Poyurovsky et al 2009 states that the mean duration of antipsychotic treatment prior to the study was 6 weeks

Funded by the Stanley Medical Research Institute, conflicts of interest not reported

Study conducted from 2003 to 2006 in Israel

\section{Risk of bias}

\begin{tabular}{lll}
\hline Bias & Authors' judgement & Support for judgement \\
\hline $\begin{array}{l}\text { Random sequence genera- } \\
\text { tion (selection bias) }\end{array}$ & Low risk & $\begin{array}{l}\text { "The participants were allocated according to entries on a table of random } \\
\text { numbers" }\end{array}$ \\
\hline $\begin{array}{l}\text { Allocation concealment } \\
\text { (selection bias) }\end{array}$ & Unclear risk & Method not described \\
\hline
\end{tabular}

$\begin{aligned} & \text { Blinding of participants } \\ & \text { and personnel (perfor- }\end{aligned}$
Low risk

mance bias)

All outcomes

\begin{tabular}{|c|c|c|}
\hline $\begin{array}{l}\text { Blinding of outcome as- } \\
\text { sessment (detection bias) } \\
\text { All outcomes }\end{array}$ & Low risk & $\begin{array}{l}\text { "Clinical and research staff and patients were unaware of and could not deter- } \\
\text { mine the study drug assignment by appearance or otherwise...All weight mea- } \\
\text { surements were performed by a research assistant blinded to the patients' } \\
\text { treatment assignment...Clinical ratings were completed at baseline and at } \\
\text { week } 6 \text { by the same trained psychiatrist...who was blinded to the patients' } \\
\text { treatment assignment...Neuropsychological assessments were performed by } \\
\text { the psychologist...who was blind to the subjects' treatment condition and } \\
\text { clinical rating scores" }\end{array}$ \\
\hline
\end{tabular}

$\begin{array}{lll}\text { Incomplete outcome data } & \text { Low risk } & \begin{array}{l}\text { Appropriate imputation methods for weight data. Similar withdrawal rates } \\ \text { (attrition bias) }\end{array}\end{array}$

All outcomes and reasons between groups

\begin{tabular}{lll}
\hline $\begin{array}{l}\text { Selective reporting (re- } \\
\text { porting bias) }\end{array}$ & Low risk & All outcomes of interest reported \\
\hline Other bias & Low risk & No other specific biases noted \\
\hline
\end{tabular}

Sacco 2009

\begin{tabular}{ll}
\hline Methods & Allocation: randomised \\
& Blindness: double blind, parallel groups \\
& Duration: 2 weeks \\
\hline Participants & Diagnosis: schizophrenia or schizoaffective disorder with nicotine dependence \\
& $\mathrm{N}=12$ \\
Age: 18 to 59 years & Sex: not reported \\
\hline
\end{tabular}


Sacco 2009 (Continued)

History: in stable remission from active psychiatric syptomatology but baseline severity and duration of illness not reported, SCID DSM-IV diagnosis of schizophrenia or schizoaffective disorder and nicotine dependence, smoking $\geq 15$ cigarettes/day, expired breath carbon monoxide $>10$ parts per million, stable remission, stable psychotropics last 3 months, IQ > 80, VSWM, CPT, or WCST at least 1 standard deviation below mean

Exclusions: abuse or dependence of alcohol or other substances of abuse in last 3 months, other Axis I disorder, unable to give informed consent, methadone, paroxetine, fluoxetine, or quinidine, contraindications to atomoxetine, current or planning pregnancy

$\begin{array}{ll}\text { Interventions } & \text { NRI: atomoxetine } 80 \mathrm{mg} \text { daily. } \mathrm{N}=3 \\ \text { 2. NRI: atomoxetine } 40 \mathrm{mg} \text { daily. } \mathrm{N}=4 \\ \text { 3. Placebo. } \mathrm{N}=5\end{array}$

\section{Risk of bias}

\begin{tabular}{|c|c|c|}
\hline Bias & Authors' judgement & Support for judgement \\
\hline $\begin{array}{l}\text { Random sequence genera- } \\
\text { tion (selection bias) }\end{array}$ & Unclear risk & Method not described in detail, "randomized in a double-blind manner" \\
\hline $\begin{array}{l}\text { Allocation concealment } \\
\text { (selection bias) }\end{array}$ & Unclear risk & Method not described \\
\hline $\begin{array}{l}\text { Blinding of participants } \\
\text { and personnel (perfor- } \\
\text { mance bias) } \\
\text { All outcomes }\end{array}$ & Unclear risk & Method not described in detail, "double-blind, placebo-controlled" \\
\hline $\begin{array}{l}\text { Blinding of outcome as- } \\
\text { sessment (detection bias) } \\
\text { All outcomes }\end{array}$ & Unclear risk & Method not described \\
\hline $\begin{array}{l}\text { Incomplete outcome data } \\
\text { (attrition bias) } \\
\text { All outcomes }\end{array}$ & Unclear risk & Method not described \\
\hline $\begin{array}{l}\text { Selective reporting (re- } \\
\text { porting bias) }\end{array}$ & High risk & Only data for selected differences reported \\
\hline Other bias & Unclear risk & Some baseline imbalance but significance not clear \\
\hline
\end{tabular}


Schutz 2001

\begin{tabular}{ll}
\hline Methods & Allocation: randomised \\
& Blindness: double blind, parallel groups \\
Duration: 6 weeks
\end{tabular}

Diagnosis: schizophrenia
$\mathrm{N}=30$ (23 completers)
Age: mean 33 years
Sex: $2 \mathrm{~F}, 28 \mathrm{M}$
History: baseline severity mild (mean PANSS total 79) and mean duration of illness 98 months, inpa-
tients in Sterkfontein Hospital with DSM-IV schizophrenia (acute relapse $\mathrm{n}=13$; or chronic $\mathrm{n}=17$ ) on
haloperidol 5 mg daily with at least partial response, 4 weeks washout for depot or 2 weeks for other
psychotropics
Exclusions: significant medical illness or medication; psychotropics other than benzodiazepines, any
other major psychiatric diagnosis, substance abuse or more than 3 alcoholic drinks daily

Interventions

1. NRI: reboxetine $8 \mathrm{mg}$ daily in divided doses. $\mathrm{N}=12$

2. Placebo. $\mathrm{N}=11$

\begin{tabular}{|c|c|}
\hline \multirow[t]{4}{*}{ Outcomes } & $\begin{array}{l}\text { Mental state: Response rate ( } \geq 20 \% \text { reduction on PANSS), PANSS total, negative, positive, general sub- } \\
\text { scales, HRSD } † \S\end{array}$ \\
\hline & Clinical global response: CGI†§ \\
\hline & Leaving the study early \\
\hline & Adverse effects: Side-effects§, SAS†§ \\
\hline \multirow[t]{4}{*}{ Notes } & Unclear total numbers in each arm prior to withdrawals but presumed to be 15 in each \\
\hline & $\begin{array}{l}\text { Additional correspondence with author Michael Berk (MB) clarifying randomisation and blinding (see } \\
\text { below) }\end{array}$ \\
\hline & Funding and conflicts of interest not reported \\
\hline & Study conducted in South Africa but date study conducted not reported \\
\hline
\end{tabular}

\section{Risk of bias}

\begin{tabular}{lll}
\hline Bias & Authors' judgement & Support for judgement \\
\hline $\begin{array}{l}\text { Random sequence genera- } \\
\text { tion (selection bias) }\end{array}$ & Low risk & $\begin{array}{l}\text { "randomization was done by an external statistician using computer genera- } \\
\text { tion" MB }\end{array}$ \\
\hline $\begin{array}{l}\text { Allocation concealment } \\
\text { (selection bias) }\end{array}$ & Low risk & As above \\
\hline $\begin{array}{l}\text { Blinding of participants } \\
\text { and personnel (perfor- } \\
\text { mance bias) } \\
\text { All outcomes }\end{array}$ & Low risk & $\begin{array}{l}\text { "blinding was done by over encapsulation and repackaging into identical la- } \\
\text { belled containers" MB }\end{array}$ \\
\hline
\end{tabular}

Blinding of outcome as-
sessment (detection bias) $\quad$ Unclear risk Method not described


Schutz 2001 (Continued)

All outcomes

$\begin{array}{lll}\text { Incomplete outcome data } & \text { Unclear risk } & \begin{array}{l}\text { Withdrawals accounted for and balanced but missing data is not clearly } \\ \text { (attrition bias) }\end{array}\end{array}$

All outcomes

Selective reporting (re- Low risk $\quad$ All outcomes of interest reported
porting bias)

porting bias)

Other bias Low risk No other specific biases noted

\section{Shafti 2015}

\begin{tabular}{ll}
\hline Methods & Allocation: randomised \\
Blindness: double blind, parallel groups \\
Duration: 12 weeks \\
\hline
\end{tabular}

\begin{tabular}{|c|c|}
\hline \multirow[t]{6}{*}{ Participants } & Diagnosis: schizophrenia \\
\hline & $N=50$ \\
\hline & Age: mean 40 years \\
\hline & Sex: All male \\
\hline & $\begin{array}{l}\text { History: baseline severity severely ill (SANS } 80 \text { ) and mean duration of illness } 9 \text { years, long-term inpa- } \\
\text { tients in the Razi Psychiatric Hospital with DSM-IV schizophrenia and chronic negative symptoms, > } 2 \\
\text { years negative symptoms, SANS } \geq 66 \text {, SAPS } \leq 96, \text { SAS } \leq 10,2 \text { weeks wash-out then start haloperidol } 5 \\
\text { mg/day }\end{array}$ \\
\hline & $\begin{array}{l}\text { Exclusions: schizoaffective disorder, major depression, mental retardation, neurological disorders, } \\
\text { medical complications, severe aggressiveness, medically deaf or mute, long-acting depot in last } 6 \\
\text { months, atypical antipsychotics, antidepressants, lithium }\end{array}$ \\
\hline \multirow[t]{2}{*}{ Interventions } & 1. NRI: reboxetine $4 \mathrm{mg}$ daily. $\mathrm{N}=25$ \\
\hline & 2. Placebo. $\mathrm{N}=25$ \\
\hline \multirow[t]{7}{*}{ Outcomes } & $\begin{array}{l}\text { Mental state: SANS score and response rate (reduction } \geq 20 \% \text { ), SANS subscales, subscale response } \\
\text { rates, SAPS } †\end{array}$ \\
\hline & Leaving the study early \\
\hline & Adverse effects: side-effects§ \\
\hline & Unable to use (not reported) \\
\hline & Mental state: HRSD \\
\hline & Cognitive functioning: MMSE \\
\hline & Adverse effects: SAS \\
\hline \multirow[t]{2}{*}{ Notes } & $\begin{array}{l}\text { Additional correspondence with author Saeed Shoja Shafti (SS) clarifying randomisation and blinding } \\
\text { (see below) }\end{array}$ \\
\hline & Funded by Razi Psychiatric Hospital, no interests declared \\
\hline
\end{tabular}




\section{Risk of bias}

\section{Bias}

\section{Authors' judgement Support for judgement}

Random sequence genera- Unclear risk tion (selection bias)
"Randomization was based on the number of file (bed) that had been determined for each patient upon admission in the hospital...they had been divided or designated one by one and by chance into two different groups until completion of the groups

"every patient had the same chance to be in the treatment or control arms', and there was no pre-determined plan for dividing the numbers into groups, for example, based on 'even' or 'odd' , or alternately and else" SS

Non-concurrence in ratings (PM: unclear risk; MP: low risk) and overall unclear risk agreed after discussion

Allocation concealment Unclear risk Method not described
(selection bias)

Blinding of participants Low risk and personnel (performance bias)

All outcomes

\begin{abstract}
"The tablets were prescribed while previously inserted into empty and similar capsules, which were prepared in this regard, to make patients blind regarding the procedure. The evaluator (a psychiatrist) also remained unaware concerning the above mentioned panel and the type of medications prescribed for each group

"The assessor, staff and patients were unaware regarding the prescribed drugs, which were arranged by an extra colleague, who was supervisor and manager of the tryout" SS
\end{abstract}

\begin{tabular}{|c|c|c|}
\hline $\begin{array}{l}\text { Blinding of outcome as- } \\
\text { sessment (detection bias) } \\
\text { All outcomes }\end{array}$ & Low risk & $\begin{array}{l}\text { "The assessor, staff and patients were unaware regarding the prescribed } \\
\text { drugs, which were arranged by an extra colleague, who was supervisor and } \\
\text { manager of the tryout" SS }\end{array}$ \\
\hline
\end{tabular}
"there was no dropout during the assessment"
Incomplete outcome data Low risk
(attrition bias)

All outcomes

\begin{tabular}{lll}
\hline $\begin{array}{l}\text { Selective reporting (re- } \\
\text { porting bias) }\end{array}$ & Low risk & $\begin{array}{l}\text { Some selective reporting of outcomes (SAS and MMSE in particular) but prima- } \\
\text { ry outcomes reported and no significant outcomes of interest excluded }\end{array}$ \\
\hline Other bias & Low risk & No other specific biases noted \\
\hline
\end{tabular}

Usall 2014

\begin{tabular}{ll} 
Methods & Allocation: randomised \\
& Blindness: double blind, parallel groups \\
& Duration: 6 months \\
\hline Participants & Diagnosis: schizophrenia \\
& N $=90$ (64 completers) \\
Age: 18 to 65 years (mean 42 years) \\
Sex: $13 \mathrm{~F}, 67 \mathrm{M}$
\end{tabular}


Usall 2014 (Continued)

History: baseline severity mildly ill (mean PANSS total 73) and mean duration of illness 16 years, multicentre study (10 centres in Spain), DSM-IV, stable treatment with olanzapine or risperidone for 60 days, 1 or more negative symptom with severity score $>4$ on PANSS negative scale

Exclusions: substance misuse or dependence in last 6 months, learning disability, antidepressant or mood stabiliser use in last 4 months, use of antipsychotics other than olanzapine or risperidone, or use of multiple antipsychotics, HRSD > 20, pregnancy or lactation, severe renal failure, history of haemorrhagic disorders, allergy or intolerance to citalopram or reboxetine

Interventions
2. PRI: reboxetine $4 \mathrm{mg}$ daily for 1 week then $8 \mathrm{mg}$ daily. $\mathrm{N}=34$
3. Active control: citalopram $15 \mathrm{mg}$ daily for 1 week then $30 \mathrm{mg}$ daily. $\mathrm{N}=23$

Outcomes

Mental state: PANSS total, positive, negative, and general subscales, SANS total, affective flatten-

ing/blunting, alogia, avolition/apathy, anhedonia/asociality, attentional impairment subscales†§

Leaving the study early

Adverse effects: acute exacerbation of psychosis side-effect§

Unable to use

Outcomes at 12 weeks as sample size not reported

Notes

Hypnotic use of levomepromazine up to $100 \mathrm{mg}$, clotiapine $40 \mathrm{mg}$, chlorpromazine $100 \mathrm{mg}$, or quetiapine $200 \mathrm{mg}$ daily was allowed

Benzodiazepine and biperiden use permitted. No changes to antipsychotic doses throughout trial

Statistically significant difference in baseline psychiatric comorbidity between reboxetine and placebo arms $(21 \%$ vs $47 \%)$

Funded by Fondo de Investigación Sanitario, conflicts of interest include honoraria, grants, and advisory boards for multiple pharmaceutical companies and funding bodies including Eli Lilly and Pfizer

Study conducted 2008 to 2011 in Spain

\section{Risk of bias}

\begin{tabular}{|c|c|c|}
\hline Bias & Authors' judgement & Support for judgement \\
\hline $\begin{array}{l}\text { Random sequence genera- } \\
\text { tion (selection bias) }\end{array}$ & Low risk & "randomly assigned...on the basis of a random number list" \\
\hline $\begin{array}{l}\text { Allocation concealment } \\
\text { (selection bias) }\end{array}$ & Low risk & "randomly assigned by the...trial pharmacy" \\
\hline $\begin{array}{l}\text { Blinding of participants } \\
\text { and personnel (perfor- } \\
\text { mance bias) } \\
\text { All outcomes }\end{array}$ & Low risk & $\begin{array}{l}\text { "...tablets were prepared so they were identical in appearance. All study per- } \\
\text { sonnel and participants remained blind to treatment assignment for the dura- } \\
\text { tion of the study" }\end{array}$ \\
\hline
\end{tabular}

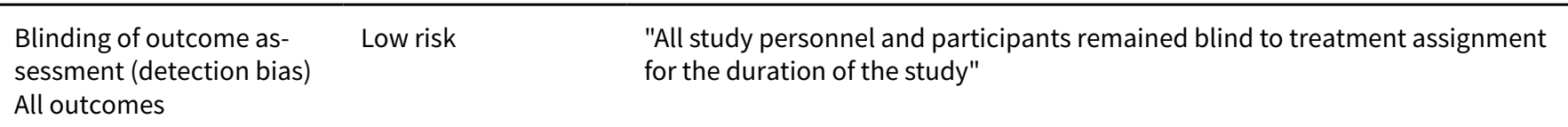

\begin{tabular}{|c|c|c|}
\hline $\begin{array}{l}\text { Incomplete outcome data } \\
\text { (attrition bias) }\end{array}$ & Unclear risk & $\begin{array}{l}\text { Similar number of withdrawals between groups but differences in reasons for } \\
\text { withdrawals, in particular due to exacerbation of illness with reboxetine com- }\end{array}$ \\
\hline
\end{tabular}
All outcomes 
Usall 2014 (Continued)

pared to placebo ( 2 vs 0 ), but unclear if this is sufficient to make a significant difference to results

\begin{tabular}{lll}
\hline $\begin{array}{l}\text { Selective reporting (re- } \\
\text { porting bias) }\end{array}$ & Low risk & All significant outcomes of interest reported \\
\hline Other bias & Low risk & No other specific biases noted \\
\hline
\end{tabular}

Yu 2012

Methods $\quad$ Allocation: randomised
Blindness: open, parallel groups
Duration: 16 weeks

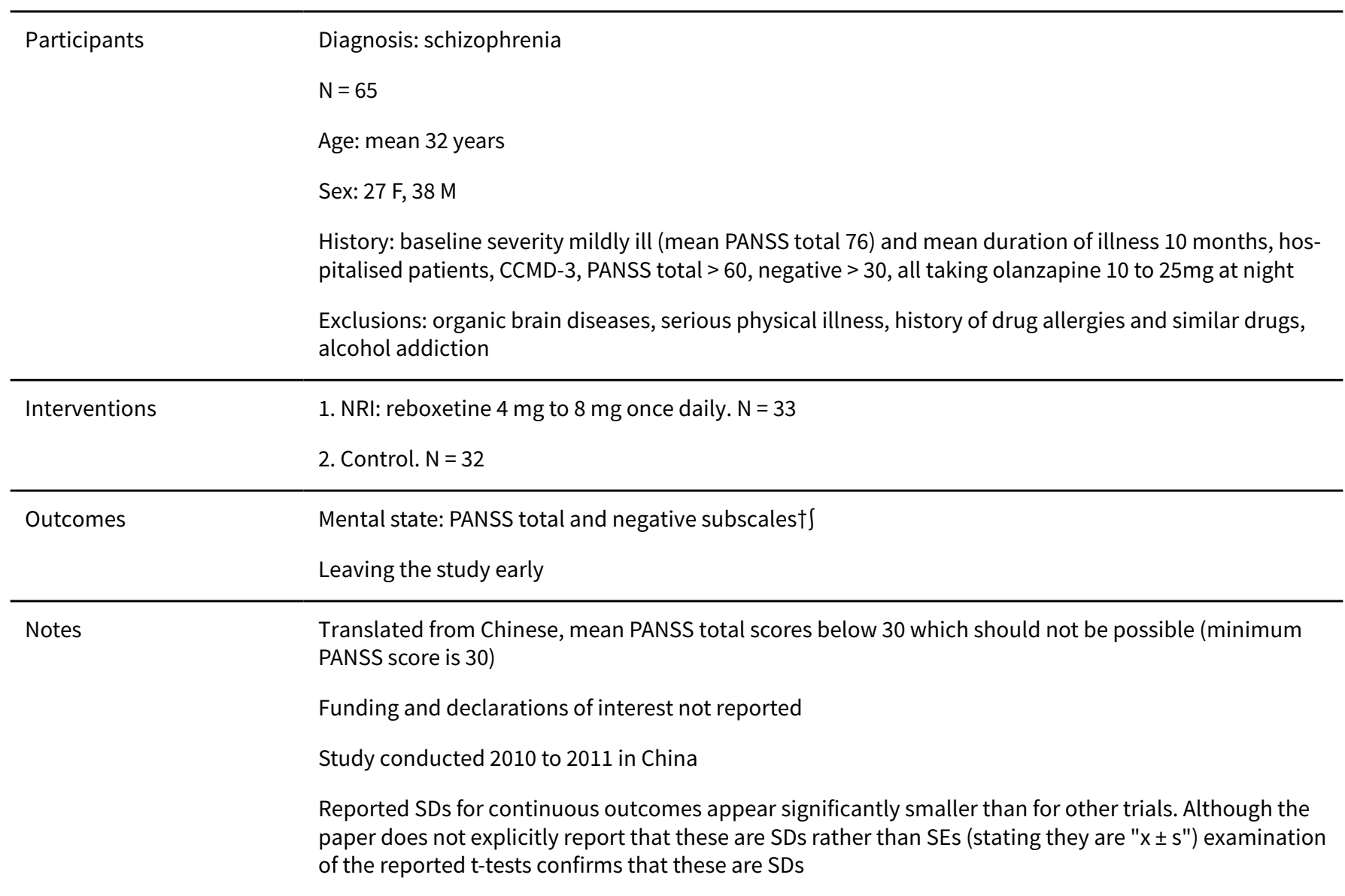

\section{Risk of bias}

\begin{tabular}{lll}
\hline Bias & Authors' judgement & Support for judgement \\
\hline $\begin{array}{l}\text { Random sequence genera- } \\
\text { tion (selection bias) }\end{array}$ & Unclear risk & "open randomized controlled study" \\
\hline $\begin{array}{l}\text { Allocation concealment } \\
\text { (selection bias) }\end{array}$ & High risk & "open randomized controlled study" \\
\hline
\end{tabular}


Yu 2012 (Continued)
Blinding of participants
High risk
"open randomized controlled study" and personnel (performance bias)

All outcomes

\begin{tabular}{lll}
\hline $\begin{array}{l}\text { Blinding of outcome as- } \\
\text { sessment (detection bias) } \\
\text { All outcomes }\end{array}$ & High risk & "open randomized controlled study" \\
\hline $\begin{array}{l}\text { Incomplete outcome data } \\
\text { (attrition bias) } \\
\text { All outcomes }\end{array}$ & Unclear risk & No explanations for withdrawals \\
\hline $\begin{array}{l}\text { Selective reporting (re- } \\
\text { porting bias) }\end{array}$ & High risk & Not all outcomes reported including response rates \\
\hline \begin{tabular}{l} 
Other bias \\
\hline
\end{tabular} & High risk & Methodological concerns such as PANSS total scores below minimum of 30 \\
\hline
\end{tabular}

Zhao 2013

\begin{tabular}{ll}
\hline Methods & Allocation: randomised \\
Blindness: double blind, parallel groups \\
Duration: 24 weeks
\end{tabular}

Participants

Diagnosis: schizophrenia

$\mathrm{N}=110$ (107 after withdrawals)

Age: 18 to 55 years (mean 43 years)

Sex: All male

History: baseline severity mildly ill (mean PANSS total 74), duration of illness not reported, ICD-10, inpatients on clozapine and stable for 1 year, metabolic syndrome by IDF 2005 criteria, clozapine dose unchanged during trial, patients discharged from trial if they use additional psychotropics, PANSS negative $\geq 22$

Exclusions: use of other antipsychotics or psychotropics in the last year, substance misuse, abnormalities on blood tests or physical examination, treatment for metabolic syndrome, enrolment in other medical research, organic brain disease, severe physical illness, epilepsy

Interventions
2. PRI: reboxetine $4 \mathrm{mg}$ daily increased to $8 \mathrm{mg}$ daily after 1 week. $\mathrm{N}=53$

Outcomes

Mental state: PANSS total, positive, negative, general scores†§

Adverse effects: Side-effects, weight

Unable to use

Leaving the study early: not reported by treatment arm

\section{Notes}

Translated from Chinese. Benzodiazepine use for anxiety or insomnia

Funding and declarations of interest not reported 
Zhao 2013 (Continued)

Study conducted 2011 to 2012 in China

Reported SDs for continuous outcomes appear significantly smaller than for other trials. Although the paper does not explicitly report that these are SDs rather than SEs (stating they are " $\bar{x} \pm s$ ") examination of the reported t-tests confirms that these are SDs

\section{Risk of bias}

\begin{tabular}{|c|c|c|}
\hline Bias & Authors' judgement & Support for judgement \\
\hline $\begin{array}{l}\text { Random sequence genera- } \\
\text { tion (selection bias) }\end{array}$ & Unclear risk & Method not described \\
\hline $\begin{array}{l}\text { Allocation concealment } \\
\text { (selection bias) }\end{array}$ & Unclear risk & Method not described \\
\hline $\begin{array}{l}\text { Blinding of participants } \\
\text { and personnel (perfor- } \\
\text { mance bias) } \\
\text { All outcomes }\end{array}$ & Unclear risk & Method not described \\
\hline $\begin{array}{l}\text { Blinding of outcome as- } \\
\text { sessment (detection bias) } \\
\text { All outcomes }\end{array}$ & Unclear risk & Method not described \\
\hline $\begin{array}{l}\text { Incomplete outcome data } \\
\text { (attrition bias) } \\
\text { All outcomes }\end{array}$ & Unclear risk & Withdrawals not reported by treatment arm \\
\hline $\begin{array}{l}\text { Selective reporting (re- } \\
\text { porting bias) }\end{array}$ & Low risk & Reports all pre-specified outcomes \\
\hline Other bias & Low risk & No other specific biases noted \\
\hline
\end{tabular}

Abbreviations: AIMS - Abnormal Involuntary Movement Scale; BAS - Barnes Akathisia Scale; BPRS - Brief Psychiatric Rating Scale; CCMD Chinese Classification of Mental Disorders; CGI - Clinical Global Impression; DSM - Diagnostic and Statistical Manual of Mental Disorders; HRSD - Hamilton Rating Scale for Depression; ITT - intention to treat; LOCF - last observation carried forward; MADRS - MontgomeryÅsberg Depression Rating Scale; MAOI - Monoamine oxidase inhibitor; MMSE - Mini-Mental State Examination; PANSS - Positive and Negative Syndrome Scale; RBANS - Repeatable Battery for the Assessment of Neuropsychological Status; SAPS - Scale for the Assessment of Positive Symptoms; SANS - Scale for the Assessment of Negative Symptoms; SAS - Simpson-Angus Scale; SCID - Structured Clinical Interview for DSM; SEC - Side-Effects Checklist

$\dagger$ Published outcome measures

$\ddagger$ Composite score not previously published but individual scores are

$\S$ Self-report or independence of raters not stated

$¥$ Independent raters

$\int$ Raters not independent

Characteristics of excluded studies [ordered by study ID]

Study Reason for exclusion

Allocation: randomised parallel group

Participants: schizophrenia or schizophreniform disorder

Intervention: reboxetine or placebo 


\begin{tabular}{ll}
\hline Study & Reason for exclusion \\
\hline $\begin{array}{l}\text { Outcomes: data from Poyurovsky } 2003 \text { and Poyurovsky } 2007 \text { were combined to report metabolic } \\
\text { and hormonal measures which have not been defined as outcomes in our review }\end{array}$ \\
\hline Apud 2007a & $\begin{array}{l}\text { Allocation: randomised cross-over trial, stratified by coMT genotype } \\
\text { Intervention: atomoxetine or placebo }\end{array}$ \\
& $\begin{array}{l}\text { Outcomes: terminated early due to slow recruitment and low priority, only } 4 \text { patients recruited and } \\
\text { completed and no data collected }\end{array}$ \\
\hline Allocation: randomised parallel group \\
Participants: schizophrenia, schizophreniform disorder, schizoaffective disorder, or psychosis not \\
otherwise specified \\
Intervention: citalopram or placebo
\end{tabular}

\begin{tabular}{|c|c|}
\hline Hou 2007 & $\begin{array}{l}\text { Allocation: randomised parallel group } \\
\text { Participants: schizophrenia } \\
\text { Intervention: citalopram or placebo }\end{array}$ \\
\hline Mueller 2005 & $\begin{array}{l}\text { Allocation: } 2 \text { randomised parallel group trials (1-2) } \\
\text { Participants: } \\
\text { 1. schizophrenia (on risperidone) } \\
\text { 2. depression (on reboxetine) } \\
\text { Intervention: celecoxib or placebo }\end{array}$ \\
\hline Poyurovsky 2013 & $\begin{array}{l}\text { Allocation: randomised parallel group } \\
\text { Participants: schizophrenia } \\
\text { Intervention: reboxetine plus betahistine or placebo } \\
\text { Excluded as betahistine use was not equally distributed across treatment and control groups }\end{array}$ \\
\hline Salokangas 1997 & $\begin{array}{l}\text { Allocation: randomised parallel group } \\
\text { Participants: schizophrenia } \\
\text { Intervention: citalopram or placebo }\end{array}$ \\
\hline Shafti 2004 & $\begin{array}{l}\text { Allocation: } 3 \text { randomised parallel group trials (1-3) } \\
\text { Participants: schizophrenia } \\
\text { Intervention: } \\
\text { 1. clomipramine, alprazolam, citalopram or placebo } \\
\text { 2. bromocriptine, fluoxetine, nortriptyline or placebo } \\
\text { 3. fluvoxamine, maprotiline or placebo } \\
\text { Excluded as none of the active compounds is a selective NRI }\end{array}$ \\
\hline
\end{tabular}


Characteristics of studies awaiting assessment [ordered by study ID]

Shekhar 2005

\begin{tabular}{ll}
\hline Methods & 52 week randomised parallel group placebo-controlled trial \\
\hline Participants & Recruitment target of 126 patients with treatment-resistant schizophrenia on clozapine \\
\hline Interventions & Atomoxetine (up to $40 \mathrm{mg}$ daily) or placebo \\
\hline Outcomes & Symptom scales (e.g. PANSS, CGI), cognitive testing (BACS), side-effect scales (e.g. AIMS), weight \\
\hline Notes & Trial terminated but no further information on results could be obtained \\
\hline
\end{tabular}

Tamminga 2009

\begin{tabular}{ll}
\hline Methods & 12 week randomised factorial design parallel group placebo-controlled trial \\
\hline Participants & 119 patients with schizophrenia recruited \\
\hline Interventions & \begin{tabular}{l} 
1. Atomoxetine ( 40 mg twice daily) plus cognitive remediation \\
2. Atomoxetine plus remediation control \\
3. Placebo plus cognitive remediation \\
4. Placebo plus remediation control \\
\hline Outcomes
\end{tabular} $\begin{array}{l}\text { Symptom scales (e.g. PANSS, CGI), cognitive testing, physical examination (e.g. ECG, weight), neu- } \\
\text { roimaging (fMRI) }\end{array}$ \\
\hline Notes & Trial completed but no further information on results could be obtained \\
\hline
\end{tabular}

Characteristics of ongoing studies [ordered by study ID]

Baranchik 2006

\begin{tabular}{|c|c|}
\hline Trial name or title & Reboxetine Adjuvant Therapy for the Treatment of Schizophrenia \\
\hline Methods & 6 week randomised parallel group placebo-controlled trial \\
\hline Participants & Recruitment target of 30 patients with schizophrenia (aged 18 to 65 years) \\
\hline Interventions & Reboxetine (titrated up to $4 \mathrm{mg}$ twice daily) or placebo \\
\hline Outcomes & Symptom scales (e.g. PANSS, SANS) and cognitive testing (CogScan) \\
\hline Starting date & March 2006 \\
\hline Contact information & Stanislav Baranchik, Abarbanel Medical Mental Health Center, Israel \\
\hline
\end{tabular}




\section{DATA AND ANALYSES}

\section{Comparison 1. SELECTIVE NORADRENALINE REUPTAKE INHIBITORS verSUS PLACEBO}

\begin{tabular}{|c|c|c|c|c|}
\hline Outcome or subgroup title & No. of studies & $\begin{array}{l}\text { No. of partici- } \\
\text { pants }\end{array}$ & Statistical method & Effect size \\
\hline $\begin{array}{l}1 \text { Primary outcome - A. Mental state: } \\
\text { Specific - clinically significant response } \\
\text { or improvement in negative symptoms } \\
\text { (SANS, high=worse) - short term ( } 12 \\
\text { weeks) }\end{array}$ & 1 & 50 & $\begin{array}{l}\text { Risk Ratio (M-H, Random, } \\
95 \% \mathrm{Cl} \text { ) }\end{array}$ & $3.17[1.52,6.58]$ \\
\hline $\begin{array}{l}2 \text { Primary outcome - B. Clinical global } \\
\text { response: Clinically significant response } \\
\text { or improvement in global status (CGI-S, } \\
\text { high=worse) - short term ( } 4 \text { weeks) }\end{array}$ & 1 & 28 & $\begin{array}{l}\text { Risk Ratio (M-H, Random, } \\
95 \% \mathrm{Cl} \text { ) }\end{array}$ & $0.99[0.45,2.20]$ \\
\hline $\begin{array}{l}3 \text { Mental state: } 1 \text {. General - clinically sig- } \\
\text { nificant response or improvement in } \\
\text { general/overall symptoms as defined in } \\
\text { each study - short term ( } 2-12 \text { weeks) }\end{array}$ & 2 & 58 & $\begin{array}{l}\text { Risk Ratio (M-H, Random, } \\
95 \% \mathrm{Cl} \text { ) }\end{array}$ & $1.15[0.64,2.09]$ \\
\hline $\begin{array}{l}4 \text { Mental state: } 2 \text { a. General: Average gen- } \\
\text { eral/overall symptoms score (various } \\
\text { scales, high=worse) - short term ( } 2 \text { - } 12 \\
\text { weeks) }\end{array}$ & 7 & & $\begin{array}{l}\text { Mean Difference (IV, Ran- } \\
\text { dom, } 95 \% \mathrm{CI} \text { ) }\end{array}$ & Subtotals only \\
\hline 4.1 BPRS total & 1 & 23 & $\begin{array}{l}\text { Mean Difference (IV, Ran- } \\
\text { dom, } 95 \% \mathrm{CI} \text { ) }\end{array}$ & $1.5[-6.64,9.64]$ \\
\hline 4.2 PANSS general & 5 & 294 & $\begin{array}{l}\text { Mean Difference (IV, Ran- } \\
\text { dom, } 95 \% \mathrm{CI} \text { ) }\end{array}$ & $-2.17[-3.93,-0.40]$ \\
\hline 4.3 PANSS total & 4 & 308 & $\begin{array}{l}\text { Mean Difference (IV, Ran- } \\
\text { dom, } 95 \% \mathrm{CI} \text { ) }\end{array}$ & $-2.84[-5.28,-0.40]$ \\
\hline $\begin{array}{l}5 \text { Mental state: } 2 \text { b. General: Average gen- } \\
\text { eral/overall symptoms score (various } \\
\text { scales, high=worse) - medium term (13 - } \\
26 \text { weeks) }\end{array}$ & 4 & & $\begin{array}{l}\text { Mean Difference (IV, Ran- } \\
\text { dom, } 95 \% \mathrm{CI} \text { ) }\end{array}$ & Subtotals only \\
\hline 5.1 BPRS total & 1 & 26 & $\begin{array}{l}\text { Mean Difference (IV, Ran- } \\
\text { dom, } 95 \% \mathrm{CI} \text { ) }\end{array}$ & $-1.4[-7.08,4.28]$ \\
\hline 5.2 PANSS general & 2 & 154 & $\begin{array}{l}\text { Mean Difference (IV, Ran- } \\
\text { dom, } 95 \% \mathrm{CI} \text { ) }\end{array}$ & $-2.90[-7.57,1.77]$ \\
\hline 5.3 PANSS total & 3 & 219 & $\begin{array}{l}\text { Mean Difference (IV, Ran- } \\
\text { dom, } 95 \% \mathrm{CI} \text { ) }\end{array}$ & $-3.67[-10.07,2.72]$ \\
\hline $\begin{array}{l}6 \text { Mental state: } 3 a \text {. Specific: Aver- } \\
\text { age symptoms score (various scales, } \\
\text { high=worse) - short term ( } 2 \text { - } 12 \text { weeks) }\end{array}$ & 10 & & $\begin{array}{l}\text { Mean Difference (IV, Ran- } \\
\text { dom, } 95 \% \mathrm{CI} \text { ) }\end{array}$ & Subtotals only \\
\hline $6.1 \operatorname{mood}(B P R S)$ & 1 & 23 & $\begin{array}{l}\text { Mean Difference (IV, Ran- } \\
\text { dom, } 95 \% \mathrm{CI} \text { ) }\end{array}$ & $0.2[-2.60,3.00]$ \\
\hline
\end{tabular}




\begin{tabular}{|c|c|c|c|c|}
\hline Outcome or subgroup title & No. of studies & $\begin{array}{l}\text { No. of partici- } \\
\text { pants }\end{array}$ & Statistical method & Effect size \\
\hline $6.2 \operatorname{mood}(\mathrm{HRSD})$ & 3 & 114 & $\begin{array}{l}\text { Mean Difference (IV, Ran- } \\
\text { dom, } 95 \% \mathrm{CI} \text { ) }\end{array}$ & $-2.37[-4.29,-0.45]$ \\
\hline 6.3 negative (PANSS negative) & 6 & 359 & $\begin{array}{l}\text { Mean Difference (IV, Ran- } \\
\text { dom, } 95 \% \mathrm{CI} \text { ) }\end{array}$ & $-0.99[-2.53,0.56]$ \\
\hline 6.4 negative (SANS) & 3 & 129 & $\begin{array}{l}\text { Mean Difference (IV, Ran- } \\
\text { dom, } 95 \% \mathrm{CI} \text { ) }\end{array}$ & $-2.47[-6.22,1.28]$ \\
\hline 6.5 negative (SANS - modified) & 1 & 23 & $\begin{array}{l}\text { Mean Difference (IV, Ran- } \\
\text { dom, } 95 \% \mathrm{CI} \text { ) }\end{array}$ & $-5.70[-18.01,6.61]$ \\
\hline 6.6 positive (BPRS) & 1 & 23 & $\begin{array}{l}\text { Mean Difference (IV, Ran- } \\
\text { dom, } 95 \% \mathrm{CI} \text { ) }\end{array}$ & $1.60[-2.59,5.79]$ \\
\hline 6.7 positive (PANSS positive) & 5 & 294 & $\begin{array}{l}\text { Mean Difference (IV, Ran- } \\
\text { dom, } 95 \% \mathrm{CI} \text { ) }\end{array}$ & $-0.16[-0.96,0.63]$ \\
\hline 6.8 positive (SAPS) & 3 & 129 & $\begin{array}{l}\text { Mean Difference (IV, Ran- } \\
\text { dom, } 95 \% \mathrm{CI} \text { ) }\end{array}$ & $0.73[-1.29,2.74]$ \\
\hline $\begin{array}{l}7 \text { Mental state: } 3 b \text {. Specific: Aver- } \\
\text { age symptoms score (various scales, } \\
\text { high=worse) - medium term (13 - } 26 \\
\text { weeks) }\end{array}$ & 3 & & $\begin{array}{l}\text { Mean Difference (IV, Ran- } \\
\text { dom, } 95 \% \mathrm{CI} \text { ) }\end{array}$ & Subtotals only \\
\hline 7.1 negative (PANSS negative) & 3 & 219 & $\begin{array}{l}\text { Mean Difference (IV, Ran- } \\
\text { dom, } 95 \% \mathrm{CI} \text { ) }\end{array}$ & $-3.25[-4.04,-2.47]$ \\
\hline 7.2 negative (SANS) & 1 & 47 & $\begin{array}{l}\text { Mean Difference (IV, Ran- } \\
\text { dom, } 95 \% \mathrm{CI} \text { ) }\end{array}$ & $-7.12[-19.39,5.15]$ \\
\hline 7.3 positive (PANSS positive) & 2 & 154 & $\begin{array}{l}\text { Mean Difference (IV, Ran- } \\
\text { dom, } 95 \% \mathrm{CI} \text { ) }\end{array}$ & $-0.14[-1.30,1.02]$ \\
\hline $\begin{array}{l}8 \text { Mental state: } 4 \text { a. General and spe- } \\
\text { cific: Average score (various scales, } \\
\text { high=worse) - skewed results - short } \\
\text { term ( } 2 \text { - } 12 \text { weeks) }\end{array}$ & & & Other data & No numeric data \\
\hline $\begin{array}{l}8.1 \text { general - overall symptoms (BPRS } \\
\text { total) }\end{array}$ & & & Other data & No numeric data \\
\hline $\begin{array}{l}8.2 \text { general - overall symptoms (PANSS } \\
\text { general) }\end{array}$ & & & Other data & No numeric data \\
\hline $\begin{array}{l}8.3 \text { general - overall symptoms (PANSS } \\
\text { total) }\end{array}$ & & & Other data & No numeric data \\
\hline $\begin{array}{l}8.4 \text { specific - mood (BPRS anxiety/de- } \\
\text { pression) }\end{array}$ & & & Other data & No numeric data \\
\hline 8.5 specific - mood (HRSD) & & & Other data & No numeric data \\
\hline 8.6 specific - negative symptoms (SANS) & & & Other data & No numeric data \\
\hline
\end{tabular}




\section{Outcome or subgroup title \\ 8.7 specific - negative symptoms (PANSS negative)}

8.8 specific - positive symptoms (BPRS positive)

\begin{tabular}{|c|c|c|c|c|}
\hline $\begin{array}{l}8.9 \text { specific - positive symptoms (PANSS } \\
\text { positive) }\end{array}$ & & & Other data & No numeric data \\
\hline 8.10 specific - positive symptoms (SAPS) & & & Other data & No numeric data \\
\hline $\begin{array}{l}9 \text { Mental state: } 4 b . \text { General and spe- } \\
\text { cific: Average score (various scales, } \\
\text { high=worse) - skewed results - medium } \\
\text { term (13 - } 26 \text { weeks) }\end{array}$ & & & Other data & No numeric data \\
\hline $\begin{array}{l}9.1 \text { general - overall symptoms (BPRS } \\
\text { total) }\end{array}$ & & & Other data & No numeric data \\
\hline $\begin{array}{l}9.2 \text { general - overall symptoms (PANSS } \\
\text { general) }\end{array}$ & & & Other data & No numeric data \\
\hline $\begin{array}{l}9.3 \text { general - overall symptoms (PANSS } \\
\text { total) }\end{array}$ & & & Other data & No numeric data \\
\hline $\begin{array}{l}9.4 \text { specific - negative symptoms (PANSS } \\
\text { negative) }\end{array}$ & & & Other data & No numeric data \\
\hline 9.5 specific - negative symptoms (SANS) & & & Other data & No numeric data \\
\hline $\begin{array}{l}9.6 \text { specific - positive symptoms (PANSS } \\
\text { positive) }\end{array}$ & & & Other data & No numeric data \\
\hline $\begin{array}{l}10 \text { Cognitive functioning: } 1 \text {. General - av- } \\
\text { erage composite cognitive functioning } \\
\text { score (SMD) }\end{array}$ & 5 & & $\begin{array}{l}\text { Std. Mean Difference (Ran- } \\
\text { dom, } 95 \% \mathrm{Cl} \text { ) }\end{array}$ & Subtotals only \\
\hline 10.1 short term ( 2 - 12 weeks) & 4 & 180 & $\begin{array}{l}\text { Std. Mean Difference (Ran- } \\
\text { dom, } 95 \% \mathrm{Cl} \text { ) }\end{array}$ & $0.04[-0.28,0.36]$ \\
\hline 10.2 medium term (13 - 26 weeks) & 1 & 26 & $\begin{array}{l}\text { Std. Mean Difference (Ran- } \\
\text { dom, } 95 \% \mathrm{Cl} \text { ) }\end{array}$ & $-0.66[-1.46,0.13]$ \\
\hline $\begin{array}{l}11 \text { Cognitive functioning: } 2 \text {. Specific - } \\
\text { average individual scores (SMD) - short } \\
\text { term ( } 2 \text { - } 12 \text { weeks) }\end{array}$ & 4 & & $\begin{array}{l}\text { Std. Mean Difference (Ran- } \\
\text { dom, } 95 \% \mathrm{Cl} \text { ) }\end{array}$ & Subtotals only \\
\hline 11.1 working memory & 2 & 53 & $\begin{array}{l}\text { Std. Mean Difference (Ran- } \\
\text { dom, } 95 \% \mathrm{Cl} \text { ) }\end{array}$ & $-0.11[-0.89,0.67]$ \\
\hline 11.2 reasoning/problem solving & 3 & 158 & $\begin{array}{l}\text { Std. Mean Difference (Ran- } \\
\text { dom, } 95 \% \mathrm{Cl} \text { ) }\end{array}$ & $0.03[-0.28,0.34]$ \\
\hline 11.3 speed of processing & 4 & 177 & $\begin{array}{l}\text { Std. Mean Difference (Ran- } \\
\text { dom, } 95 \% \mathrm{Cl} \text { ) }\end{array}$ & $0.08[-0.21,0.38]$ \\
\hline
\end{tabular}

\section{No. of partici- Statistical method Effect size}

pants

Other data

No numeric data

Other data

No numeric data 


\begin{tabular}{|c|c|c|c|c|}
\hline Outcome or subgroup title & No. of studies & $\begin{array}{l}\text { No. of partici- } \\
\text { pants }\end{array}$ & Statistical method & Effect size \\
\hline 11.4 attention & 3 & 161 & $\begin{array}{l}\text { Std. Mean Difference (Ran- } \\
\text { dom, } 95 \% \mathrm{Cl} \text { ) }\end{array}$ & $-0.01[-0.51,0.48]$ \\
\hline 11.5 verbal learning/memory & 4 & 181 & $\begin{array}{l}\text { Std. Mean Difference (Ran- } \\
\text { dom, } 95 \% \mathrm{Cl} \text { ) }\end{array}$ & $0.01[-0.31,0.32]$ \\
\hline 11.6 visual learning/memory & 1 & 22 & $\begin{array}{l}\text { Std. Mean Difference (Ran- } \\
\text { dom, } 95 \% \mathrm{Cl} \text { ) }\end{array}$ & $-0.44[-1.29,0.41]$ \\
\hline $\begin{array}{l}12 \text { Quality of life: Average quality of life } \\
\text { score (GQOLI- } 74 \text {, high=better) - short } \\
\text { term ( } 2 \text { - } 12 \text { weeks) }\end{array}$ & 1 & & $\begin{array}{l}\text { Mean Difference (IV, Ran- } \\
\text { dom, } 95 \% \mathrm{CI} \text { ) }\end{array}$ & Subtotals only \\
\hline 12.1 general - total & 1 & 114 & $\begin{array}{l}\text { Mean Difference (IV, Ran- } \\
\text { dom, } 95 \% \mathrm{CI} \text { ) }\end{array}$ & $9.36[7.89,10.83]$ \\
\hline 12.2 specific - well being - material & 1 & 114 & $\begin{array}{l}\text { Mean Difference (IV, Ran- } \\
\text { dom, } 95 \% \mathrm{CI} \text { ) }\end{array}$ & $0.21[-2.34,2.76]$ \\
\hline 12.3 specific - well being - physical & 1 & 114 & $\begin{array}{l}\text { Mean Difference (IV, Ran- } \\
\text { dom, } 95 \% \mathrm{CI} \text { ) }\end{array}$ & $0.68[-1.35,2.71]$ \\
\hline 12.4 specific - well being - psychological & 1 & 114 & $\begin{array}{l}\text { Mean Difference (IV, Ran- } \\
\text { dom, } 95 \% \mathrm{CI} \text { ) }\end{array}$ & $10.0[8.01,11.99]$ \\
\hline 12.5 specific - well being - social & 1 & 114 & $\begin{array}{l}\text { Mean Difference (IV, Ran- } \\
\text { dom, } 95 \% \mathrm{CI} \text { ) }\end{array}$ & $10.02[8.03,12.01]$ \\
\hline $\begin{array}{l}13 \text { Clinical global response: } 1 \text { a. Aver- } \\
\text { age clinical global status score (CGI-S, } \\
\text { high=worse) - short term ( } 2 \text { - } 12 \text { weeks) }\end{array}$ & 5 & 160 & $\begin{array}{l}\text { Mean Difference (IV, Ran- } \\
\text { dom, } 95 \% \mathrm{CI} \text { ) }\end{array}$ & $-0.03[-0.35,0.28]$ \\
\hline
\end{tabular}

\begin{tabular}{|c|c|c|c|c|}
\hline $\begin{array}{l}14 \text { Clinical global response: } 1 \text { b. Aver- } \\
\text { age clinical global status score (various } \\
\text { scales, high=worse) - short term ( } 2 \text { - } 12 \\
\text { weeks) - skewed results }\end{array}$ & & & Other data & No numeric data \\
\hline 14.1 CGI-I & & & Other data & No numeric data \\
\hline 14.2 CGI-S & & & Other data & No numeric data \\
\hline $\begin{array}{l}15 \text { Leaving the study early: } 1 \text { a. Short } \\
\text { term ( } 2 \text { - } 12 \text { weeks) }\end{array}$ & 9 & & $\begin{array}{l}\text { Risk Ratio (M-H, Random, } \\
95 \% \mathrm{Cl} \text { ) }\end{array}$ & Subtotals only \\
\hline 15.1 any reason & 9 & 401 & $\begin{array}{l}\text { Risk Ratio (M-H, Random, } \\
95 \% \mathrm{Cl} \text { ) }\end{array}$ & $0.94[0.63,1.39]$ \\
\hline 15.2 due to adverse effects & 4 & 178 & $\begin{array}{l}\text { Risk Ratio (M-H, Random, } \\
95 \% \mathrm{Cl} \text { ) }\end{array}$ & $2.08[0.70,6.21]$ \\
\hline 15.3 due to psychiatric symptoms & 3 & 183 & $\begin{array}{l}\text { Risk Ratio (M-H, Random, } \\
95 \% \mathrm{Cl} \text { ) }\end{array}$ & $0.97[0.44,2.13]$ \\
\hline $\begin{array}{l}16 \text { Leaving the study early: } 1 \mathrm{~b} \text {. Medium } \\
\text { term ( } 13-26 \text { weeks) }\end{array}$ & 3 & & $\begin{array}{l}\text { Risk Ratio (M-H, Random, } \\
95 \% \mathrm{Cl} \text { ) }\end{array}$ & Subtotals only \\
\hline
\end{tabular}




\begin{tabular}{|c|c|c|c|c|}
\hline Outcome or subgroup title & No. of studies & $\begin{array}{l}\text { No. of partici- } \\
\text { pants }\end{array}$ & Statistical method & Effect size \\
\hline 16.1 any reason & 3 & 169 & $\begin{array}{l}\text { Risk Ratio (M-H, Random, } \\
95 \% \mathrm{Cl})\end{array}$ & $0.92[0.52,1.62]$ \\
\hline 16.2 due to adverse effects & 1 & 33 & $\begin{array}{l}\text { Risk Ratio (M-H, Random, } \\
95 \% \mathrm{Cl})\end{array}$ & $1.47[0.31,6.95]$ \\
\hline 16.3 due to psychiatric symptoms & 1 & 49 & $\begin{array}{l}\text { Risk Ratio (M-H, Random, } \\
95 \% \mathrm{Cl})\end{array}$ & $4.44[0.22,88.04]$ \\
\hline $\begin{array}{l}17 \text { Adverse effects: } 1 . \text { General - short } \\
\text { term (binary, } 2 \text { - } 12 \text { weeks) }\end{array}$ & 5 & & $\begin{array}{l}\text { Risk Ratio (M-H, Random, } \\
95 \% \mathrm{Cl})\end{array}$ & Subtotals only \\
\hline 17.1 any & 3 & 199 & $\begin{array}{l}\text { Risk Ratio (M-H, Random, } \\
95 \% \mathrm{Cl})\end{array}$ & $3.49[0.14,90.29]$ \\
\hline 17.2 serious & 3 & 271 & $\begin{array}{l}\text { Risk Ratio (M-H, Random, } \\
95 \% \mathrm{Cl})\end{array}$ & $2.95[0.47,18.36]$ \\
\hline $\begin{array}{l}18 \text { Adverse effects: } 2 \text { a.i. Specific - short } \\
\text { term (binary, } 2 \text { - } 12 \text { weeks) }\end{array}$ & 7 & & $\begin{array}{l}\text { Risk Ratio (M-H, Random, } \\
95 \% \mathrm{Cl})\end{array}$ & Subtotals only \\
\hline 18.1 anticholinergic - constipation & 2 & 146 & $\begin{array}{l}\text { Risk Ratio (M-H, Random, } \\
95 \% \mathrm{Cl})\end{array}$ & $0.74[0.24,2.29]$ \\
\hline 18.2 anticholinergic - dry mouth & 3 & 176 & $\begin{array}{l}\text { Risk Ratio (M-H, Random, } \\
95 \% \mathrm{Cl})\end{array}$ & $3.46[1.40,8.53]$ \\
\hline 18.3 anticholinergic - impotence & 1 & 30 & $\begin{array}{l}\text { Risk Ratio (M-H, Random, } \\
95 \% \mathrm{Cl})\end{array}$ & $3.0[0.13,68.26]$ \\
\hline 18.4 cardiovascular - tachycardia & 1 & 114 & $\begin{array}{l}\text { Risk Ratio (M-H, Random, } \\
95 \% \mathrm{Cl})\end{array}$ & $3.33[0.97,11.48]$ \\
\hline 18.5 central nervious system - anorexia & 1 & 32 & $\begin{array}{l}\text { Risk Ratio (M-H, Random, } \\
95 \% \mathrm{Cl})\end{array}$ & $0.14[0.01,2.56]$ \\
\hline 18.6 central nervous system - dizziness & 3 & 176 & $\begin{array}{l}\text { Risk Ratio (M-H, Random, } \\
95 \% \mathrm{Cl})\end{array}$ & $1.52[0.54,4.31]$ \\
\hline 18.7 central nervous system - fatigue & 2 & 62 & $\begin{array}{l}\text { Risk Ratio (M-H, Random, } \\
95 \% \mathrm{Cl})\end{array}$ & $1.00[0.12,8.60]$ \\
\hline 18.8 central nervous system - headache & 1 & 121 & $\begin{array}{l}\text { Risk Ratio (M-H, Random, } \\
95 \% \mathrm{Cl})\end{array}$ & $1.23[0.52,2.90]$ \\
\hline 18.9 central nervous system - insomnia & 2 & 146 & $\begin{array}{l}\text { Risk Ratio (M-H, Random, } \\
95 \% \mathrm{Cl})\end{array}$ & $4.79[0.85,26.84]$ \\
\hline 18.10 central nervous system - sedation & 2 & 58 & $\begin{array}{l}\text { Risk Ratio (M-H, Random, } \\
95 \% \mathrm{Cl})\end{array}$ & $0.74[0.28,1.94]$ \\
\hline 18.11 extrapyramidal - acute dystonia & 1 & 30 & $\begin{array}{l}\text { Risk Ratio (M-H, Random, } \\
95 \% \mathrm{Cl})\end{array}$ & $1.0[0.07,14.55]$ \\
\hline
\end{tabular}




\begin{tabular}{|c|c|c|c|c|}
\hline Outcome or subgroup title & No. of studies & $\begin{array}{l}\text { No. of partici- } \\
\text { pants }\end{array}$ & Statistical method & Effect size \\
\hline 18.12 extrapyramidal - akathisia & 3 & 88 & $\begin{array}{l}\text { Risk Ratio (M-H, Random, } \\
95 \% \mathrm{CI})\end{array}$ & $0.87[0.25,3.09]$ \\
\hline 18.13 extrapyramidal - hypersalivation & 1 & 30 & $\begin{array}{l}\text { Risk Ratio (M-H, Random, } \\
95 \% \mathrm{Cl})\end{array}$ & $0.33[0.01,7.58]$ \\
\hline 18.14 extrapyramidal - parkinsonism & 1 & 30 & $\begin{array}{l}\text { Risk Ratio (M-H, Random, } \\
95 \% \mathrm{Cl})\end{array}$ & $1.8[0.79,4.11]$ \\
\hline 18.15 extrapyramidal - tremor & 1 & 32 & $\begin{array}{l}\text { Risk Ratio (M-H, Random, } \\
95 \% \mathrm{Cl} \text { ) }\end{array}$ & $1.0[0.24,4.23]$ \\
\hline 18.16 extrapyramidal - stiffness & 1 & 32 & $\begin{array}{l}\text { Risk Ratio (M-H, Random, } \\
95 \% \mathrm{Cl})\end{array}$ & $0.2[0.03,1.53]$ \\
\hline $\begin{array}{l}\text { 18.17 extrapyramidal - use of antiparkin- } \\
\text { son medication }\end{array}$ & 3 & 135 & $\begin{array}{l}\text { Risk Ratio (M-H, Random, } \\
95 \% \mathrm{Cl})\end{array}$ & $0.80[0.44,1.45]$ \\
\hline 18.18 gastrointestinal - abdominal pain & 1 & 32 & $\begin{array}{l}\text { Risk Ratio (M-H, Random, } \\
95 \% \mathrm{CI})\end{array}$ & $1.5[0.29,7.81]$ \\
\hline 18.19 gastrointestinal - nausea & 3 & 176 & $\begin{array}{l}\text { Risk Ratio (M-H, Random, } \\
95 \% \mathrm{CI})\end{array}$ & $0.49[0.10,2.41]$ \\
\hline 18.20 gastrointestinal - vomiting & 1 & 32 & $\begin{array}{l}\text { Risk Ratio (M-H, Random, } \\
95 \% \mathrm{Cl} \text { ) }\end{array}$ & $0.2[0.01,3.86]$ \\
\hline 18.21 gastrointestinal - diarrhoea & 1 & 32 & $\begin{array}{l}\text { Risk Ratio (M-H, Random, } \\
95 \% \mathrm{Cl} \text { ) }\end{array}$ & $0.2[0.01,3.86]$ \\
\hline 18.22 immune system - rash & 1 & 30 & $\begin{array}{l}\text { Risk Ratio (M-H, Random, } \\
95 \% \mathrm{Cl} \text { ) }\end{array}$ & $3.0[0.13,68.26]$ \\
\hline 18.23 immune system - sore throat & 1 & 32 & $\begin{array}{l}\text { Risk Ratio (M-H, Random, } \\
95 \% \mathrm{Cl} \text { ) }\end{array}$ & $0.2[0.01,3.86]$ \\
\hline 18.24 metabolic - weight loss & 1 & 32 & $\begin{array}{l}\text { Risk Ratio (M-H, Random, } \\
95 \% \mathrm{Cl})\end{array}$ & $0.33[0.04,2.87]$ \\
\hline 18.25 metabolic - significant weight gain & 2 & 85 & $\begin{array}{l}\text { Risk Ratio (M-H, Random, } \\
95 \% \mathrm{Cl} \text { ) }\end{array}$ & $0.38[0.20,0.73]$ \\
\hline $\begin{array}{l}19 \text { Adverse effects: } 2 \text { a.ii. Specific - } \\
\text { extrapyramidal - average change } \\
\text { score (continuous, various scales, } \\
\text { high=worse) - short term ( } 2 \text { - } 12 \text { weeks) }\end{array}$ & 2 & & $\begin{array}{l}\text { Mean Difference (IV, Ran- } \\
\text { dom, } 95 \% \mathrm{CI} \text { ) }\end{array}$ & Subtotals only \\
\hline 19.1 AIMS & 1 & 26 & $\begin{array}{l}\text { Mean Difference (IV, Ran- } \\
\text { dom, } 95 \% \mathrm{CI} \text { ) }\end{array}$ & $0.3[-2.34,2.94]$ \\
\hline 19.2 BAS & 1 & 59 & $\begin{array}{l}\text { Mean Difference (IV, Ran- } \\
\text { dom, } 95 \% \mathrm{CI} \text { ) }\end{array}$ & $-0.18[-0.65,0.29]$ \\
\hline 19.3 SAS & 2 & 85 & $\begin{array}{l}\text { Mean Difference (IV, Ran- } \\
\text { dom, } 95 \% \mathrm{CI} \text { ) }\end{array}$ & $-0.27[-1.79,1.25]$ \\
\hline
\end{tabular}




\section{Outcome or subgroup title \\ 20 Adverse effects: 2a.iii. Specific - extrapyramidal - average change score (continuous, various scales, high=worse) - short term (2 - 12 weeks) - skewed results}

\begin{tabular}{|c|c|c|c|c|}
\hline 20.1 AIMS & & & Other data & No numeric data \\
\hline 20.2 SAS & & & Other data & No numeric data \\
\hline $\begin{array}{l}21 \text { Adverse effects: } 2 \text { b.i. Specific - medi- } \\
\text { um term (binary, } 13 \text { - } 26 \text { weeks) }\end{array}$ & 2 & & $\begin{array}{l}\text { Risk Ratio (M-H, Random, } \\
95 \% \mathrm{Cl})\end{array}$ & Subtotals only \\
\hline 21.1 anticholinergic - constipation & 1 & 107 & $\begin{array}{l}\text { Risk Ratio (M-H, Random, } \\
95 \% \mathrm{Cl} \text { ) }\end{array}$ & $4.58[1.04,20.23]$ \\
\hline 21.2 anticholinergic - dry mouth & 1 & 107 & $\begin{array}{l}\text { Risk Ratio (M-H, Random, } \\
95 \% \mathrm{Cl} \text { ) }\end{array}$ & $1.53[0.27,8.78]$ \\
\hline 21.3 anticholinergic - sweating & 1 & 107 & $\begin{array}{l}\text { Risk Ratio (M-H, Random, } \\
95 \% \mathrm{Cl} \text { ) }\end{array}$ & $\begin{array}{l}13.24[0.76 \\
229.32]\end{array}$ \\
\hline 21.4 cardiovascular - QT prolongation & 1 & 37 & $\begin{array}{l}\text { Risk Ratio (M-H, Random, } \\
95 \% \mathrm{Cl} \text { ) }\end{array}$ & $3.4[0.42,27.59]$ \\
\hline 21.5 cardiovascular - tachycardia & 1 & 107 & $\begin{array}{l}\text { Risk Ratio (M-H, Random, } \\
95 \% \mathrm{Cl} \text { ) }\end{array}$ & $1.78[0.55,5.74]$ \\
\hline 21.6 central nervous system - dizziness & 1 & 107 & $\begin{array}{l}\text { Risk Ratio (M-H, Random, } \\
95 \% \mathrm{Cl})\end{array}$ & $1.36[0.32,5.78]$ \\
\hline 21.7 central nervous system - insomnia & 1 & 107 & $\begin{array}{l}\text { Risk Ratio (M-H, Random, } \\
95 \% \mathrm{Cl} \text { ) }\end{array}$ & $\begin{array}{l}13.24[0.76 \\
229.32]\end{array}$ \\
\hline 21.8 extrapyramidal - tremor & 1 & 37 & $\begin{array}{l}\text { Risk Ratio (M-H, Random, } \\
95 \% \mathrm{Cl} \text { ) }\end{array}$ & $0.43[0.20,0.89]$ \\
\hline 21.9 gastrointestinal - nausea & 1 & 107 & $\begin{array}{l}\text { Risk Ratio (M-H, Random, } \\
95 \% \mathrm{Cl} \text { ) }\end{array}$ & $5.09[0.62,42.16]$ \\
\hline $\begin{array}{l}22 \text { Adverse effects: } 2 \text { a.iv. Specific - meta- } \\
\text { bolic - average weight gain (continuous, } \\
\text { increase in kg) }\end{array}$ & 4 & & $\begin{array}{l}\text { Mean Difference (IV, Ran- } \\
\text { dom, } 95 \% \mathrm{CI} \text { ) }\end{array}$ & Subtotals only \\
\hline 22.1 short term ( 2 - 12 weeks) & 3 & 186 & $\begin{array}{l}\text { Mean Difference (IV, Ran- } \\
\text { dom, } 95 \% \mathrm{CI} \text { ) }\end{array}$ & $-2.17[-3.19,-1.15]$ \\
\hline 22.2 medium term ( 13 - 26 weeks) & 2 & 133 & $\begin{array}{l}\text { Mean Difference (IV, Ran- } \\
\text { dom, } 95 \% \mathrm{CI} \text { ) }\end{array}$ & $-3.12[-10.67,4.42]$ \\
\hline $\begin{array}{l}23 \text { Social or general functioning: Aver- } \\
\text { age social functioning score (various } \\
\text { subscales) - short-term ( } 2 \text { - } 12 \text { weeks) }\end{array}$ & 2 & & $\begin{array}{l}\text { Mean Difference (IV, Ran- } \\
\text { dom, } 95 \% \mathrm{CI} \text { ) }\end{array}$ & Subtotals only \\
\hline
\end{tabular}

pants

Other data

No numeric data

\section{No. of partici- Statistical method Effect size}




\begin{tabular}{|c|c|c|c|c|}
\hline Outcome or subgroup title & No. of studies & $\begin{array}{l}\text { No. of partici- } \\
\text { pants }\end{array}$ & Statistical method & Effect size \\
\hline $\begin{array}{l}23.1 \text { activity - activities (inverted SLOF, } \\
\text { high=worse) }\end{array}$ & 1 & 15 & $\begin{array}{l}\text { Mean Difference (IV, Ran- } \\
\text { dom, } 95 \% \mathrm{CI} \text { ) }\end{array}$ & $0.0[-3.30,3.30]$ \\
\hline $\begin{array}{l}23.2 \text { activity - hospital activity (SSPI, } \\
\text { high=worse) }\end{array}$ & 1 & 114 & $\begin{array}{l}\text { Mean Difference (IV, Ran- } \\
\text { dom, } 95 \% \mathrm{CI} \text { ) }\end{array}$ & $-0.03[-0.17,0.11]$ \\
\hline $\begin{array}{l}23.3 \text { activity - disease indoor activity } \\
\text { (SSPI, high=worse) }\end{array}$ & 1 & 114 & $\begin{array}{l}\text { Mean Difference (IV, Ran- } \\
\text { dom, } 95 \% \mathrm{CI} \text { ) }\end{array}$ & $-0.14[-0.28,-0.00]$ \\
\hline $\begin{array}{l}23.4 \text { activity - physical functioning (in- } \\
\text { verted SLOF, high=worse) }\end{array}$ & 1 & 15 & $\begin{array}{l}\text { Mean Difference (IV, Ran- } \\
\text { dom, } 95 \% \mathrm{CI} \text { ) }\end{array}$ & $-0.3[-1.12,0.52]$ \\
\hline $\begin{array}{l}23.5 \text { personal care - personal care skills } \\
\text { (inverted SLOF, high=worse) }\end{array}$ & 1 & 15 & $\begin{array}{l}\text { Mean Difference (IV, Ran- } \\
\text { dom, } 95 \% \mathrm{CI})\end{array}$ & $-0.20[-1.43,1.03]$ \\
\hline $\begin{array}{l}23.6 \text { personal care - self-care (SSPI, } \\
\text { high=worse) }\end{array}$ & 1 & 114 & $\begin{array}{l}\text { Mean Difference (IV, Ran- } \\
\text { dom, } 95 \% \mathrm{CI})\end{array}$ & $-0.03[-0.22,0.16]$ \\
\hline $\begin{array}{l}23.7 \text { personal care - social acceptability } \\
\text { (inverted SLOF, high=worse) }\end{array}$ & 1 & 15 & $\begin{array}{l}\text { Mean Difference (IV, Ran- } \\
\text { dom, } 95 \% \mathrm{Cl} \text { ) }\end{array}$ & $0.1[-0.72,0.92]$ \\
\hline $\begin{array}{l}23.8 \text { relationships - family role (SSPI, } \\
\text { high=worse) }\end{array}$ & 1 & 114 & $\begin{array}{l}\text { Mean Difference (IV, Ran- } \\
\text { dom, } 95 \% \mathrm{CI} \text { ) }\end{array}$ & $-0.09[-0.23,0.05]$ \\
\hline $\begin{array}{l}23.9 \text { relationships - interest and con- } \\
\text { cern in the external environment (SSPI, } \\
\text { high=worse) }\end{array}$ & 1 & 114 & $\begin{array}{l}\text { Mean Difference (IV, Ran- } \\
\text { dom, } 95 \% \mathrm{Cl} \text { ) }\end{array}$ & $-0.19[-0.37,-0.01]$ \\
\hline $\begin{array}{l}23.10 \text { relationships - interpersonal rela- } \\
\text { tionships (inverted SLOF, high=worse) }\end{array}$ & 1 & 15 & $\begin{array}{l}\text { Mean Difference (IV, Ran- } \\
\text { dom, } 95 \% \mathrm{Cl} \text { ) }\end{array}$ & $-1.20[-5.41,3.01]$ \\
\hline $\begin{array}{l}23.11 \text { relationships - relationships and } \\
\text { caring for others (SSPI, high=worse) }\end{array}$ & 1 & 114 & $\begin{array}{l}\text { Mean Difference (IV, Ran- } \\
\text { dom, } 95 \% \mathrm{Cl} \text { ) }\end{array}$ & $-0.09[-0.24,0.06]$ \\
\hline $\begin{array}{l}23.12 \text { relationships - sexual role (SSPI, } \\
\text { high=worse) }\end{array}$ & 1 & 114 & $\begin{array}{l}\text { Mean Difference (IV, Ran- } \\
\text { dom, } 95 \% \mathrm{Cl})\end{array}$ & $-0.03[-0.21,0.15]$ \\
\hline $\begin{array}{l}23.13 \text { relationships - social withdrawal } \\
\text { (SSPI, high=worse) }\end{array}$ & 1 & 114 & $\begin{array}{l}\text { Mean Difference (IV, Ran- } \\
\text { dom, } 95 \% \mathrm{Cl} \text { ) }\end{array}$ & $-0.32[-0.49,-0.15]$ \\
\hline $\begin{array}{l}23.14 \text { work - professional skills (SSPI, } \\
\text { high=worse) }\end{array}$ & 1 & 114 & $\begin{array}{l}\text { Mean Difference (IV, Ran- } \\
\text { dom, } 95 \% \mathrm{Cl} \text { ) }\end{array}$ & $-0.19[-0.34,-0.04]$ \\
\hline $\begin{array}{l}23.15 \text { work - responsibility and planning } \\
\text { (SSPI, high=worse) }\end{array}$ & 1 & 114 & $\begin{array}{l}\text { Mean Difference (IV, Ran- } \\
\text { dom, } 95 \% \mathrm{CI} \text { ) }\end{array}$ & $-0.03[-0.21,0.15]$ \\
\hline $\begin{array}{l}23.16 \text { work - work skills (inverted SLOF, } \\
\text { high=worse) }\end{array}$ & 1 & 15 & $\begin{array}{l}\text { Mean Difference (IV, Ran- } \\
\text { dom, } 95 \% \mathrm{Cl} \text { ) }\end{array}$ & $-3.0[-5.48,-0.52]$ \\
\hline
\end{tabular}


Analysis 1.1. Comparison 1 SELECTIVE NORADRENALINE REUPTAKE INHIBITORS versus

PLACEBO, Outcome 1 Primary outcome - A. Mental state: Specific - clinically significant response or improvement in negative symptoms (SANS, high=worse) - short term (12 weeks).

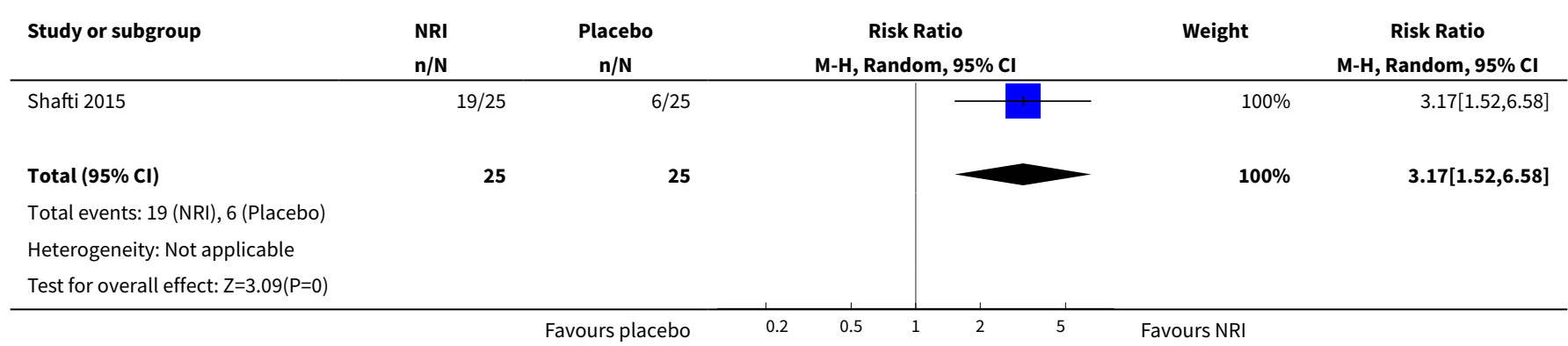

Analysis 1.2. Comparison 1 SELECTIVE NORADRENALINE REUPTAKE INHIBITORS versus PLACEBO, Outcome 2 Primary outcome - B. Clinical global response: Clinically significant response or improvement in global status (CGI-S, high=worse) - short term (4 weeks).

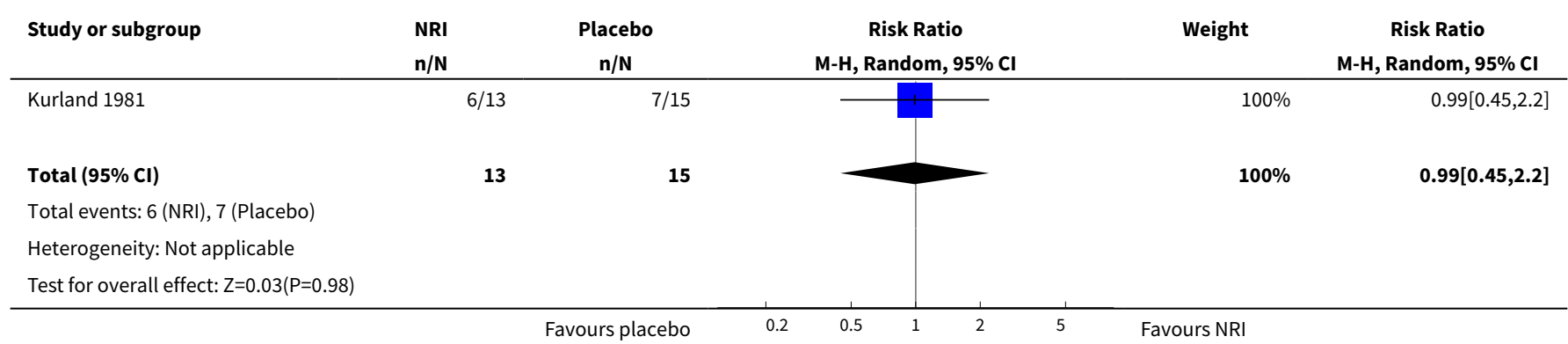

Analysis 1.3. Comparison 1 SELECTIVE NORADRENALINE REUPTAKE INHIBITORS

versus PLACEBO, Outcome 3 Mental state: 1 . General - clinically significant response or improvement in general/overall symptoms as defined in each study - short term (2-12 weeks).

\begin{tabular}{|c|c|c|c|c|c|}
\hline Study or subgroup & $\begin{array}{l}\text { NRI } \\
\mathrm{n} / \mathrm{N}\end{array}$ & $\begin{array}{c}\text { Placebo } \\
\mathbf{n} / \mathbf{N}\end{array}$ & $\begin{array}{c}\text { Risk Ratio } \\
\text { M-H, Random, } 95 \% \mathrm{Cl}\end{array}$ & Weight & $\begin{array}{c}\text { Risk Ratio } \\
\text { M-H, Random, 95\% Cl }\end{array}$ \\
\hline Kurland 1981 & $6 / 13$ & $7 / 15$ & +1 & $55.8 \%$ & $0.99[0.45,2.2]$ \\
\hline Schutz 2001 & $7 / 15$ & $5 / 15$ & -1 & $44.2 \%$ & $1.4[0.57,3.43]$ \\
\hline Total $(95 \% \mathrm{Cl})$ & 28 & 30 & & $100 \%$ & $1.15[0.64,2.09]$ \\
\hline \multicolumn{6}{|c|}{ Total events: 13 (NRI), 12 (Placebo) } \\
\hline \multicolumn{6}{|c|}{ Heterogeneity: $\operatorname{Tau}^{2}=0 ; \mathrm{Chi}^{2}=0.32, \mathrm{df}=1(\mathrm{P}=0.57) ; \mathrm{I}^{2}=0 \%$} \\
\hline Test for overall effect & & & & & \\
\hline
\end{tabular}


Analysis 1.4. Comparison 1 SELECTIVE NORADRENALINE REUPTAKE INHIBITORS versus PLACEBO, Outcome 4 Mental state: 2a. General: Average general/overall symptoms score (various scales, high=worse) - short term (2 - 12 weeks).

\begin{tabular}{|c|c|c|c|c|c|c|c|}
\hline \multirow[t]{2}{*}{ Study or subgroup } & \multicolumn{2}{|c|}{ NRI } & \multicolumn{2}{|c|}{ Placebo } & \multirow{2}{*}{$\begin{array}{l}\text { Mean Difference } \\
\text { Random, } 95 \% \mathrm{CI}\end{array}$} & \multirow[t]{2}{*}{ Weight } & \multirow{2}{*}{$\begin{array}{l}\text { Mean Difference } \\
\text { Random, } 95 \% \mathrm{Cl}\end{array}$} \\
\hline & $\mathbf{N}$ & $\operatorname{Mean}(\mathrm{SD})$ & $\mathbf{N}$ & $\operatorname{Mean}(\mathrm{SD})$ & & & \\
\hline \multicolumn{8}{|l|}{ 1.4.1 BPRS total } \\
\hline Kelly 2009 & 11 & $-2.3(9)$ & 12 & $-3.8(10.9)$ & & $100 \%$ & $1.5[-6.64,9.64]$ \\
\hline Subtotal $\star \star \star ~$ & 11 & & 12 & & & $100 \%$ & $1.5[-6.64,9.64]$ \\
\hline \multicolumn{8}{|c|}{ Heterogeneity: Not applicable } \\
\hline \multicolumn{8}{|c|}{ Test for overall effect: $\mathrm{Z}=0.36(\mathrm{P}=0.72)$} \\
\hline \multicolumn{8}{|l|}{ 1.4.2 PANSS general } \\
\hline Friedman 2008 & 7 & $-4.7(5.1)$ & 8 & $-4.4(8.9)$ & & $5.4 \%$ & $-0.3[-7.53,6.93]$ \\
\hline Hinkelmann 2013 & 19 & $-3.8(8.7)$ & 16 & $-5(9.4)$ & & $7.44 \%$ & $1.2[-4.83,7.23]$ \\
\hline Li 2008 & 57 & $28(3.1)$ & 57 & $29.7(5.4)$ & 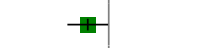 & $38.07 \%$ & $-1.68[-3.31,-0.05]$ \\
\hline Schutz 2001 & 12 & $-3.2(8.9)$ & 11 & $-3.7(7.4)$ & & $6.2 \%$ & $0.5[-6.19,7.19]$ \\
\hline Zhao 2013 & 53 & $30.3(3)$ & 54 & $34.1(3.9)$ & $\#$ & $42.88 \%$ & $-3.8[-5.12,-2.48]$ \\
\hline Subtotal $\star \star \star ~$ & 148 & & 146 & & & $100 \%$ & $-2.17[-3.93,-0.4]$ \\
\hline \multicolumn{8}{|c|}{ Heterogeneity: $\mathrm{Tau}^{2}=1.44 ; \mathrm{Chi}^{2}=7.09, \mathrm{df}=4(\mathrm{P}=0.13) ; \mathrm{I}^{2}=43.57 \%$} \\
\hline \multicolumn{8}{|c|}{ Test for overall effect: $Z=2.4(P=0.02)$} \\
\hline \multicolumn{8}{|l|}{ 1.4.3 PANSS total } \\
\hline Li 2008 & 57 & $66.5(5.9)$ & 57 & $70.7(8.7)$ & —- & $27.42 \%$ & $-4.17[-6.91,-1.43]$ \\
\hline Schutz 2001 & 12 & $-9.6(20.7)$ & 11 & $-9.7(15)$ & & $2.6 \%$ & $0.1[-14.56,14.76]$ \\
\hline Yu 2012 & 33 & $50.6(2.8)$ & 32 & $51.4(2.5)$ & \# & $37.56 \%$ & $-0.82[-2.1,0.46]$ \\
\hline Zhao 2013 & 53 & $66.9(4.6)$ & 53 & $71.2(6)$ & 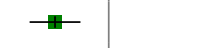 & $32.42 \%$ & $-4.3[-6.34,-2.26]$ \\
\hline Subtotal ${ }^{\star \star \star}$ & 155 & & 153 & & 8 & $100 \%$ & $-2.84[-5.28,-0.4]$ \\
\hline \multicolumn{8}{|c|}{ Heterogeneity: $\mathrm{Tau}^{2}=3.7 ; \mathrm{Chi}^{2}=10.63, \mathrm{df}=3(\mathrm{P}=0.01) ; \mathrm{I}^{2}=71.78 \%$} \\
\hline Test for overall effec & & & & & & & \\
\hline
\end{tabular}

Analysis 1.5. Comparison 1 SELECTIVE NORADRENALINE REUPTAKE INHIBITORS versus PLACEBO, Outcome 5 Mental state: $\mathbf{2 b}$. General: Average general/overall symptoms score (various scales, high=worse) - medium term (13 - 26 weeks).

\begin{tabular}{|c|c|c|c|c|c|c|c|}
\hline \multirow[t]{2}{*}{ Study or subgroup } & \multicolumn{2}{|c|}{ NRI } & \multicolumn{2}{|c|}{ Placebo } & \multirow{2}{*}{$\begin{array}{l}\text { Mean Difference } \\
\text { Random, } 95 \% \mathrm{Cl}\end{array}$} & \multirow[t]{2}{*}{ Weight } & \multirow{2}{*}{$\begin{array}{l}\text { Mean Difference } \\
\text { Random, } 95 \% \mathrm{Cl}\end{array}$} \\
\hline & $\mathbf{N}$ & $\operatorname{Mean}(S D)$ & $\mathbf{N}$ & $\operatorname{Mean}(\mathrm{SD})$ & & & \\
\hline \multicolumn{8}{|l|}{ 1.5.1 BPRS total } \\
\hline Ball 2011 & 14 & $-2.4(6.3)$ & 12 & $-1(8.2)$ & & $100 \%$ & $-1.4[-7.08,4.28]$ \\
\hline Subtotal $\star \star \star \star$ & 14 & & 12 & & & $100 \%$ & $-1.4[-7.08,4.28]$ \\
\hline \multicolumn{8}{|c|}{ Heterogeneity: Not applicable } \\
\hline \multicolumn{8}{|c|}{ Test for overall effect: $Z=0.48(P=0.63)$} \\
\hline \multicolumn{8}{|l|}{ 1.5.2 PANSS general } \\
\hline Usall 2014 & 24 & $-4.9(8.2)$ & 23 & $-5.2(9.9)$ & & $36.44 \%$ & $0.25[-4.96,5.46]$ \\
\hline Zhao 2013 & 53 & $27.9(2.9)$ & 54 & $32.6(3.8)$ & + & $63.56 \%$ & $-4.7[-5.98,-3.42]$ \\
\hline Subtotal $* \star \star$ & 77 & & 77 & & & $100 \%$ & $-2.9[-7.57,1.77]$ \\
\hline \multicolumn{8}{|c|}{ Heterogeneity: Tau $^{2}=8.5 ; \mathrm{Chi}^{2}=3.27, \mathrm{df}=1(\mathrm{P}=0.07) ; 1^{2}=69.39 \%$} \\
\hline \multicolumn{8}{|c|}{ Test for overall effect: $\mathrm{Z}=1.22(\mathrm{P}=0.22)$} \\
\hline \multicolumn{8}{|l|}{ 1.5.3 PANSS total } \\
\hline \multirow[t]{2}{*}{ Usall 2014} & 24 & $-12.1(16.7)$ & 23 & $-13(19.3)$ & 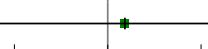 & $19.9 \%$ & $0.92[-9.41,11.25]$ \\
\hline & & & & Favours NRI & 0 & Favours | & \\
\hline
\end{tabular}




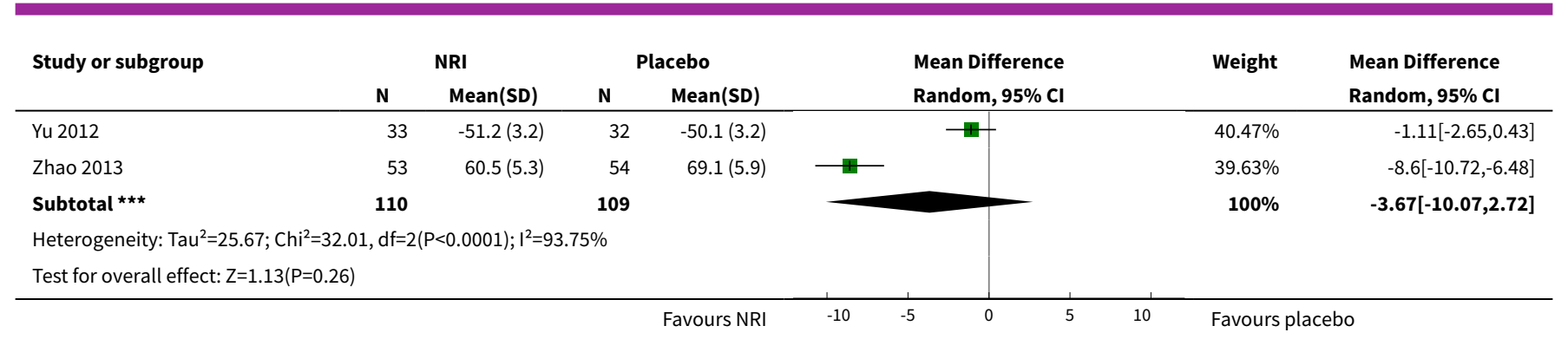
Analysis 1.6. Comparison 1 SELECTIVE NORADRENALINE REUPTAKE INHIBITORS versus PLACEBO, Outcome
6 Mental state: $3 a$. Specific: Average symptoms score (various scales, high=worse) - short term ( 2 - 12 weeks).

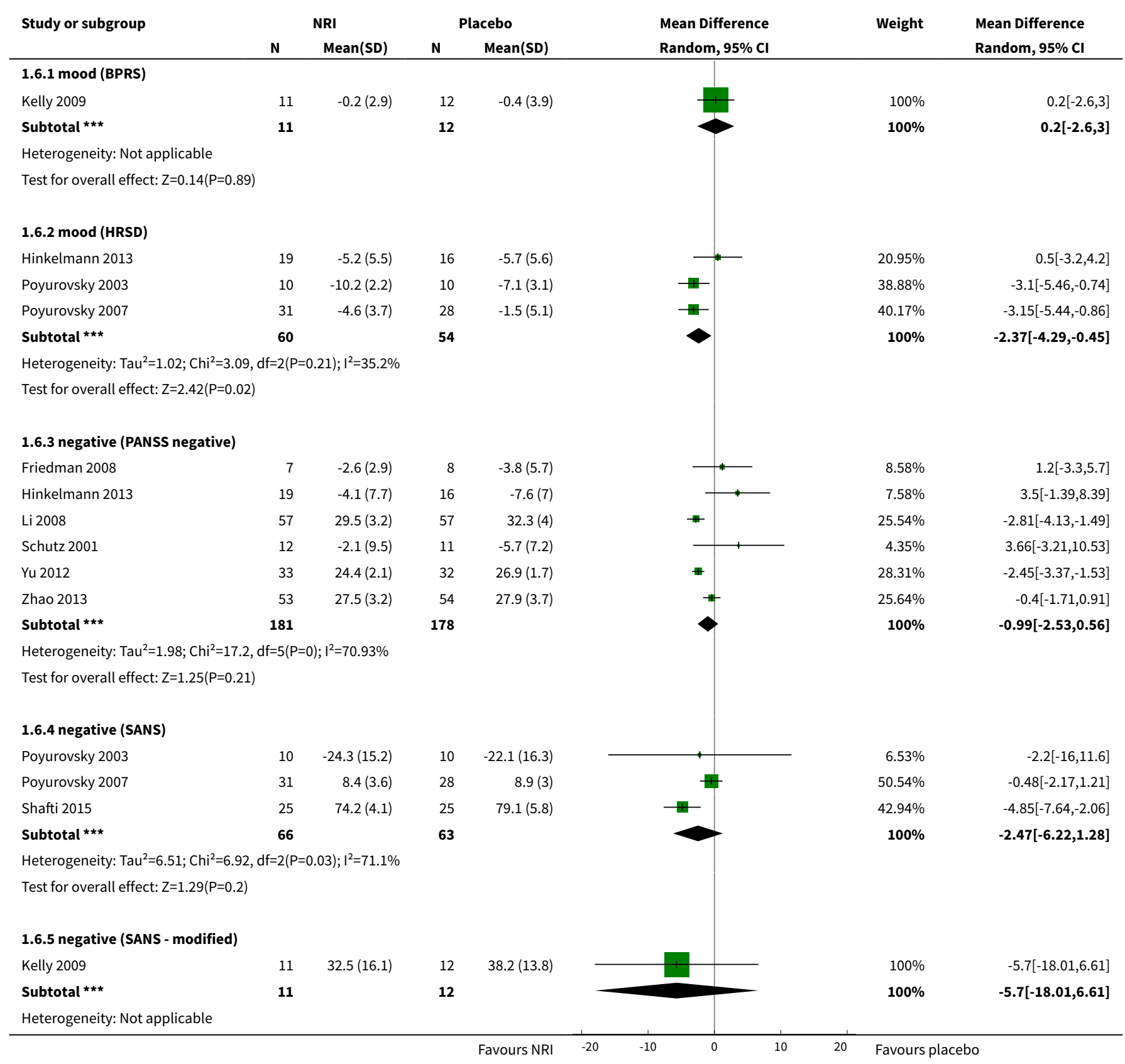




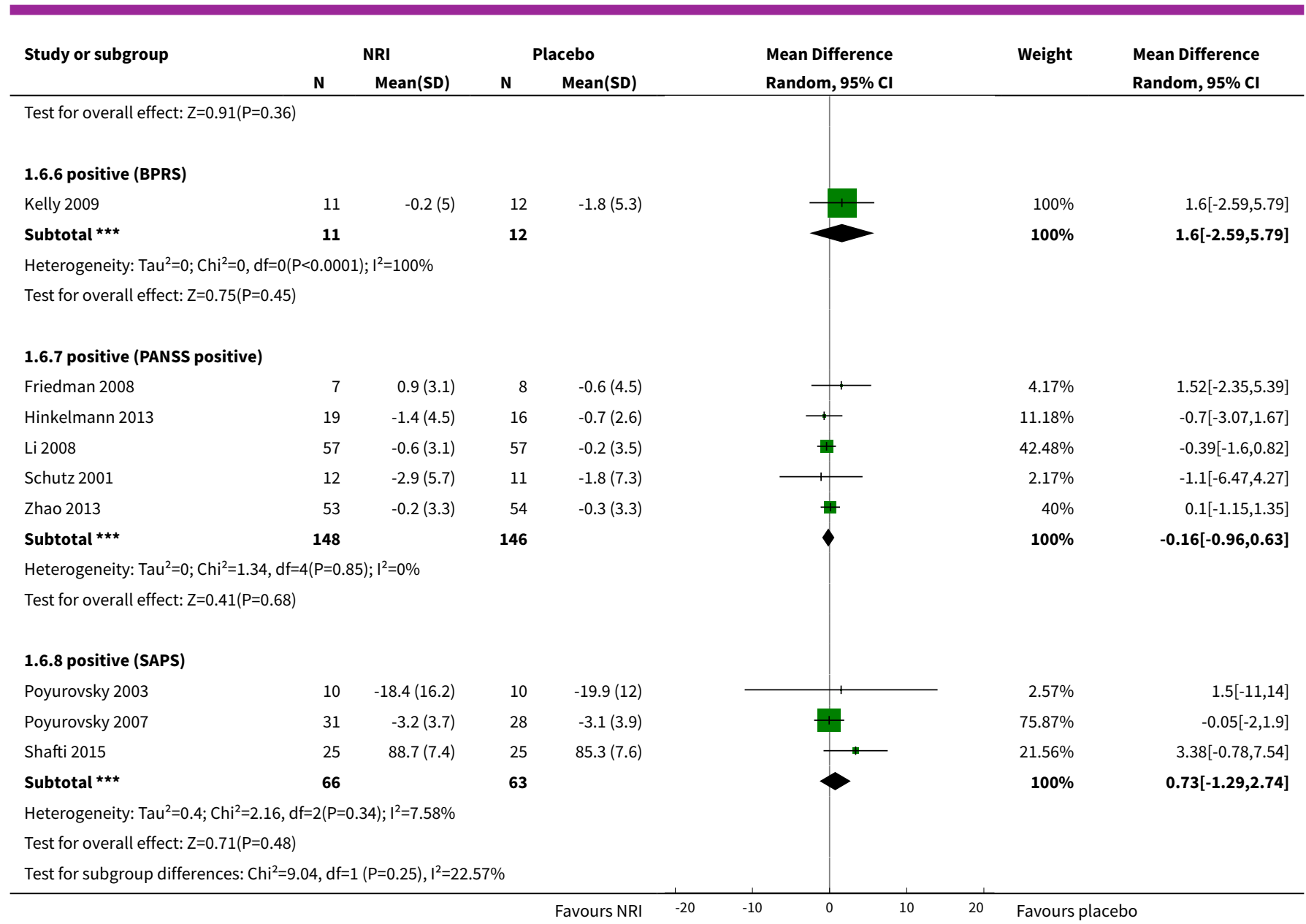

Analysis 1.7. Comparison 1 SELECTIVE NORADRENALINE REUPTAKE INHIBITORS versus PLACEBO, Outcome 7 Mental state: 3b. Specific: Average symptoms score (various scales, high=worse) - medium term (13 - 26 weeks).

\begin{tabular}{|c|c|c|c|c|c|c|c|}
\hline \multirow[t]{2}{*}{ Study or subgroup } & \multicolumn{2}{|c|}{ NRI } & \multicolumn{2}{|c|}{ Placebo } & \multirow{2}{*}{$\begin{array}{l}\text { Mean Difference } \\
\text { Random, } 95 \% \mathrm{Cl}\end{array}$} & \multirow[t]{2}{*}{ Weight } & \multirow{2}{*}{$\begin{array}{l}\text { Mean Difference } \\
\text { Random, } 95 \% \mathrm{Cl}\end{array}$} \\
\hline & $\mathbf{N}$ & Mean(SD) & $\mathbf{N}$ & $\operatorname{Mean}(S D)$ & & & \\
\hline \multicolumn{8}{|c|}{ 1.7.1 negative (PANSS negative) } \\
\hline Usall 2014 & 24 & $-5.8(7.7)$ & 23 & $-3.6(6)$ & $\longrightarrow$ & $3.97 \%$ & $-2.27[-6.21,1.67]$ \\
\hline Yu 2012 & 33 & $18.2(2.3)$ & 32 & $21.2(2.4)$ & + & $48.25 \%$ & $-3.09[-4.22,-1.96]$ \\
\hline Zhao 2013 & 53 & $23.6(3)$ & 54 & $27.1(3)$ & \# & $47.78 \%$ & $-3.5[-4.64,-2.36]$ \\
\hline Subtotal $* \star \star$ & 110 & & 109 & & $\diamond$ & $100 \%$ & $-3.25[-4.04,-2.47]$ \\
\hline \multicolumn{8}{|c|}{ Test for overall effect: $Z=8.11(P<0.0001)$} \\
\hline \multicolumn{8}{|c|}{ 1.7.2 negative (SANS) } \\
\hline Usall 2014 & 24 & $-14.8(23.6)$ & 23 & $-7.7(19.2)$ & & $100 \%$ & $-7.12[-19.39,5.15]$ \\
\hline 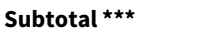 & 24 & & 23 & & & $100 \%$ & $-7.12[-19.39,5.15]$ \\
\hline \multicolumn{8}{|c|}{ Heterogeneity: Not applicable } \\
\hline \multicolumn{8}{|c|}{ 1.7.3 positive (PANSS positive) } \\
\hline Usall 2014 & 24 & $-1.3(4.8)$ & 23 & $-1.6(6)$ & $\longrightarrow$ & $13.96 \%$ & $0.24[-2.87,3.35]$ \\
\hline & & & & Favours NRI & $-10 \quad-5$ & Favours & \\
\hline
\end{tabular}




\begin{tabular}{|c|c|c|c|c|c|c|c|}
\hline \multirow[t]{2}{*}{ Study or subgroup } & \multicolumn{2}{|c|}{ NRI } & \multicolumn{2}{|c|}{ Placebo } & \multirow{2}{*}{$\begin{array}{l}\text { Mean Difference } \\
\text { Random, } 95 \% \mathrm{Cl}\end{array}$} & \multirow[t]{2}{*}{ Weight } & \multirow{2}{*}{$\begin{array}{l}\text { Mean Difference } \\
\text { Random, } 95 \% \mathrm{Cl}\end{array}$} \\
\hline & $\mathbf{N}$ & Mean(SD) & $\mathbf{N}$ & Mean(SD) & & & \\
\hline Zhao 2013 & 53 & $-0.3(3.2)$ & 54 & $-0.1(3.4)$ & & $86.04 \%$ & $-0.2[-1.45,1.05]$ \\
\hline Subtotal $\star \star \star ~$ & 77 & & 77 & & & $100 \%$ & $-0.14[-1.3,1.02]$ \\
\hline \multicolumn{8}{|c|}{ Heterogeneity: $\mathrm{Tau}^{2}=0 ; \mathrm{Chi}^{2}=0.07, \mathrm{df}=1(\mathrm{P}=0.8) ; \mathrm{I}^{2}=0 \%$} \\
\hline \multicolumn{3}{|c|}{ Test for overall effect: $\mathrm{Z}=0.23(\mathrm{P}=0.81)$} & & & & & \\
\hline
\end{tabular}

\section{Analysis 1.8. Comparison 1 SELECTIVE NORADRENALINE REUPTAKE INHIBITORS versus PLACEBO, Outcome 8 Mental state: 4a. General and specific: Average score (various scales, high=worse) - skewed results - short term ( 2 - 12 weeks).}

\begin{tabular}{|c|c|c|c|c|c|c|c|}
\hline \multicolumn{8}{|c|}{ Mental state: 4a. General and specific: Average score (various scales, high=worse) - skewed results - short term (2 - 12 weeks) } \\
\hline Study & NRI & NRI mean & NRI SD & NRI N & Placebo mean & Placebo SD & Placebo $\mathbf{N}$ \\
\hline \multicolumn{8}{|c|}{ general - overall symptoms (BPRS total) } \\
\hline Kelly 2009 & Atomoxetine & 30.2 & 8.2 & 11 & 36 & 11.6 & 12 \\
\hline \multicolumn{8}{|c|}{ general - overall symptoms (PANSS general) } \\
\hline Friedman 2008 & Atomoxetine & 26.7 & 4.4 & 10 & 32 & 8 & 10 \\
\hline Hinkelmann 2013 & Reboxetine & 28.7 & 9.4 & 19 & 28.4 & 10.1 & 16 \\
\hline Schutz 2001 & Reboxetine & 29.6 & 8.92 & 12 & 30.4 & 7.44 & 11 \\
\hline \multicolumn{8}{|c|}{ general - overall symptoms (PANSS total) } \\
\hline Schutz 2001 & Reboxetine & 67.67 & 20.66 & 12 & 70.67 & 14.96 & 11 \\
\hline \multicolumn{8}{|c|}{ specific - mood (BPRS anxiety/depression) } \\
\hline Kelly 2009 & Atomoxetine & 6.4 & 1.9 & 11 & 8.3 & 3.6 & 12 \\
\hline \multicolumn{8}{|c|}{ specific - mood (HRSD) } \\
\hline Hinkelmann 2013 & Reboxetine & 8.3 & 6.1 & 19 & 6.5 & 5.6 & 16 \\
\hline Poyurovsky 2003 & Reboxetine & 4.6 & 3.5 & 10 & 4.6 & 3.5 & 10 \\
\hline Poyurovsky 2007 & Reboxetine & 4.53 & 2 & 16 & 3 & 2 & 17 \\
\hline Schutz 2001 & Reboxetine & 6.93 & 4.95 & 12 & 6.93 & 3.17 & 11 \\
\hline \multicolumn{8}{|c|}{ specific - negative symptoms (SANS) } \\
\hline Poyurovsky 2003 & Reboxetine & 18.9 & 13.4 & 10 & 23.2 & 18.8 & 10 \\
\hline \multicolumn{8}{|c|}{ specific - negative symptoms (PANSS negative) } \\
\hline Friedman 2008 & Atomoxetine & 15.2 & 4.8 & 10 & 15.5 & 5.9 & 10 \\
\hline Hinkelmann 2013 & Reboxetine & 21.5 & 8.6 & 19 & 19.1 & 5.5 & 16 \\
\hline Schutz 2001 & Reboxetine & 25.13 & 9.53 & 12 & 23.27 & 7.21 & 11 \\
\hline \multicolumn{8}{|c|}{ specific - positive symptoms (BPRS positive) } \\
\hline Kelly 2009 & Atomoxetine & 9.8 & 4.9 & 11 & 10.3 & 5.5 & 12 \\
\hline \multicolumn{8}{|c|}{ specific - positive symptoms (PANSS positive) } \\
\hline Friedman 2008 & Atomoxetine & 13.2 & 4.5 & 10 & 14.6 & 4.5 & 10 \\
\hline Hinkelmann 2013 & Reboxetine & 10.6 & 4.6 & 19 & 8.6 & 2.4 & 16 \\
\hline Li 2008 & Reboxetine & 9.02 & 2.64 & 57 & 8.72 & 3.21 & 57 \\
\hline Schutz 2001 & Reboxetine & 14.1 & 5.68 & 12 & 15.8 & 7.29 & 11 \\
\hline Zhao 2013 & Reboxetine & 9.1 & 3.4 & 54 & 9.1 & 3.1 & 53 \\
\hline \multicolumn{8}{|c|}{ specific - positive symptoms (SAPS) } \\
\hline Poyurovsky 2003 & Reboxetine & 4.8 & 3.9 & 10 & 11.8 & 12.2 & 10 \\
\hline Poyurovsky 2007 & Reboxetine & 3.21 & 3.2 & 31 & 2.66 & 2.4 & 28 \\
\hline
\end{tabular}


Analysis 1.9. Comparison 1 SELECTIVE NORADRENALINE REUPTAKE INHIBITORS

versus PLACEBO, Outcome 9 Mental state: 4b. General and specific: Average score

(various scales, high=worse) - skewed results - medium term (13 - 26 weeks).

Mental state: 4b. General and specific: Average score (various scales, high=worse) - skewed results - medium term (13 - 26 weeks)

\begin{tabular}{|c|c|c|c|c|c|c|c|}
\hline Study & NRI & NRI mean & NRI SD & NRI N & Placebo mean & Placebo SD & Placebo N \\
\hline \multicolumn{8}{|c|}{ general - overall symptoms (BPRS total) } \\
\hline Ball 2011 & Atomoxetine & 30.8 & 5.8 & 14 & 31 & 8.9 & 12 \\
\hline \multicolumn{8}{|c|}{ general - overall symptoms (PANSS general) } \\
\hline Usall 2014 & Reboxetine & 28.83 & 8.1 & 24 & 30.09 & 7.93 & 23 \\
\hline \multicolumn{8}{|c|}{ general - overall symptoms (PANSS total) } \\
\hline Usall 2014 & Reboxetine & 59.96 & 17.89 & 24 & 62.04 & 19.9 & 23 \\
\hline Yu 2012 & Reboxetine & 24.24 & 3.54 & 33 & 26.86 & 1.66 & 32 \\
\hline \multicolumn{8}{|c|}{ specific - negative symptoms (PANSS negative) } \\
\hline Usall 2014 & Reboxetine & 19.67 & 8.41 & 24 & 22.65 & 5.54 & 23 \\
\hline \multicolumn{8}{|c|}{ specific - negative symptoms (SANS) } \\
\hline Usall 2014 & Reboxetine & 46.75 & 26.12 & 24 & 54.61 & 19.13 & 23 \\
\hline \multicolumn{8}{|c|}{ specific - positive symptoms (PANSS positive) } \\
\hline Usall 2014 & Reboxetine & 11.46 & 4.14 & 24 & 12 & 5.68 & 23 \\
\hline
\end{tabular}

Analysis 1.10. Comparison 1 SELECTIVE NORADRENALINE REUPTAKE INHIBITORS versus PLACEBO, Outcome 10 Cognitive functioning: 1 . General - average composite cognitive functioning score (SMD).

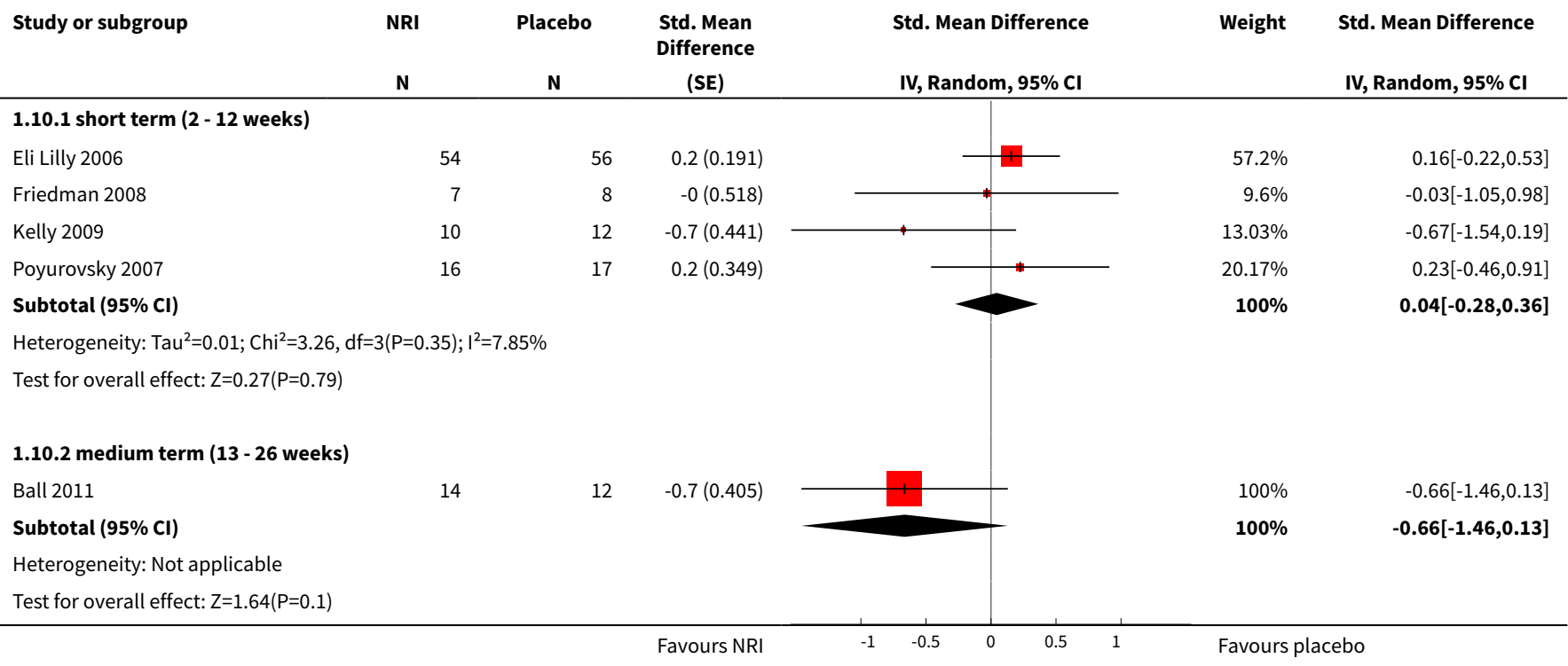

Analysis 1.11. Comparison 1 SELECTIVE NORADRENALINE REUPTAKE INHIBITORS versus PLACEBO, Outcome 11 Cognitive functioning: 2 . Specific - average individual scores (SMD) - short term (2 - 12 weeks).

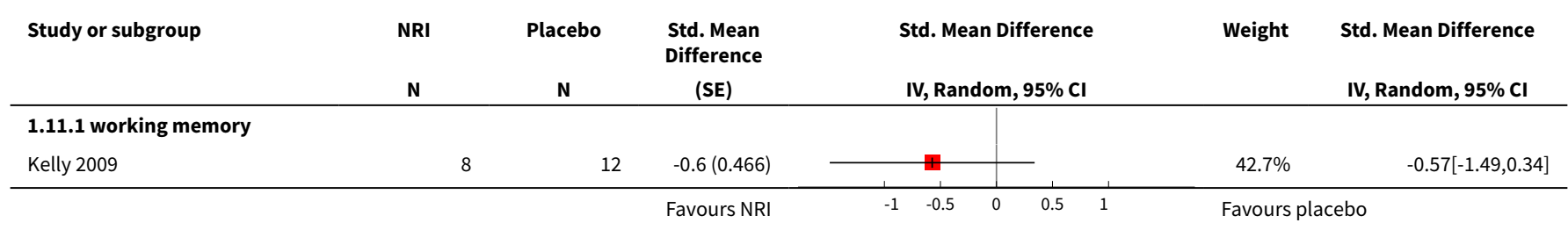




\begin{tabular}{|c|c|c|c|c|c|c|}
\hline \multirow[t]{2}{*}{ Study or subgroup } & NRI & Placebo & \multirow[t]{2}{*}{$\begin{array}{l}\text { Std. Mean } \\
\text { Difference } \\
\text { (SE) } \\
\end{array}$} & Std. Mean Difference & Weight & \multirow{2}{*}{$\begin{array}{l}\text { Std. Mean Difference } \\
\text { IV, Random, 95\% CI }\end{array}$} \\
\hline & $\mathbf{N}$ & $\mathbf{N}$ & & \multicolumn{2}{|l|}{ IV, Random, $95 \% \mathrm{CI}$} & \\
\hline Poyurovsky 2007 & 16 & 17 & $0.2(0.35)$ & & $57.3 \%$ & $0.23[-0.45,0.92]$ \\
\hline Subtotal $(95 \% \mathrm{CI})$ & & & & & $100 \%$ & $-0.11[-0.89,0.67]$ \\
\hline \multicolumn{7}{|c|}{ Heterogeneity: $\mathrm{Tau}^{2}=0.16 ; \mathrm{Chi}^{2}=1.92, \mathrm{df}=1(\mathrm{P}=0.17) ; \mathrm{I}^{2}=47.94 \%$} \\
\hline \multicolumn{7}{|c|}{ 1.11.2 reasoning/problem solving } \\
\hline Eli Lilly 2006 & 54 & 56 & $0.1(0.191)$ & & $69.82 \%$ & $0.05[-0.32,0.42]$ \\
\hline Friedman 2008 & 7 & 8 & $-0.4(0.523)$ & & $9.3 \%$ & $-0.38[-1.4,0.65]$ \\
\hline Poyurovsky 2007 & 17 & 16 & $0.1(0.349)$ & & $20.88 \%$ & $0.14[-0.54,0.82]$ \\
\hline Subtotal $(95 \% \mathrm{Cl})$ & & & & & $100 \%$ & $0.03[-0.28,0.34]$ \\
\hline \multicolumn{7}{|c|}{ Heterogeneity: Tau $^{2}=0 ; \mathrm{Chi}^{2}=0.72, \mathrm{df}=2(\mathrm{P}=0.7) ; \mathrm{I}^{2}=0 \%$} \\
\hline \multicolumn{7}{|c|}{ Test for overall effect: $Z=0.19(P=0.85)$} \\
\hline \multicolumn{7}{|c|}{ 1.11.3 speed of processing } \\
\hline Eli Lilly 2006 & 54 & 55 & $0.1(0.192)$ & & $61.82 \%$ & $0.05[-0.32,0.43]$ \\
\hline Friedman 2008 & 7 & 8 & $0.1(0.518)$ & $*$ & $8.45 \%$ & $0.14[-0.88,1.15]$ \\
\hline Kelly 2009 & 9 & 11 & $-0.1(0.45)$ & & $11.22 \%$ & $-0.1[-0.98,0.78]$ \\
\hline Poyurovsky 2007 & 16 & 17 & $0.3(0.35)$ & & $18.52 \%$ & $0.28[-0.41,0.96]$ \\
\hline Subtotal $(95 \% \mathrm{Cl})$ & & & & & $100 \%$ & $0.08[-0.21,0.38]$ \\
\hline \multicolumn{7}{|c|}{ Heterogeneity: $\mathrm{Tau}^{2}=0 ; \mathrm{Chi}^{2}=0.51, \mathrm{df}=3(\mathrm{P}=0.92) ; \mathrm{I}^{2}=0 \%$} \\
\hline \multicolumn{7}{|c|}{ Test for overall effect: $Z=0.56(P=0.58)$} \\
\hline \multicolumn{7}{|l|}{ 1.11.4 attention } \\
\hline Eli Lilly 2006 & 55 & 52 & $0.3(0.194)$ & & $48.53 \%$ & $0.27[-0.11,0.65]$ \\
\hline Subtotal $(95 \% \mathrm{Cl})$ & & & & & $100 \%$ & $-0.01[-0.51,0.48]$ \\
\hline \multicolumn{7}{|c|}{ Heterogeneity: $\mathrm{Tau}^{2}=0.1 ; \mathrm{Chi}^{2}=3.88, \mathrm{df}=2(\mathrm{P}=0.14) ; \mathrm{I}^{2}=48.4 \%$} \\
\hline \multicolumn{7}{|c|}{ Test for overall effect: $Z=0.06(P=0.95)$} \\
\hline \multicolumn{7}{|c|}{ 1.11.5 verbal learning/memory } \\
\hline Eli Lilly 2006 & 55 & 56 & $0(0.19)$ & & $57.99 \%$ & $0.02[-0.35,0.39]$ \\
\hline Friedman 2008 & 7 & 8 & $-0.1(0.518)$ & & $9.42 \%$ & $-0.08[-1.09,0.94]$ \\
\hline Kelly 2009 & 10 & 12 & $-0.6(0.438)$ & $\rightarrow$ & $12.97 \%$ & $-0.59[-1.45,0.27]$ \\
\hline Poyurovsky 2007 & 16 & 17 & $0.4(0.352)$ & & $19.61 \%$ & $0.41[-0.28,1.1]$ \\
\hline Subtotal $(95 \% \mathrm{CI})$ & & & & & $100 \%$ & $0.01[-0.31,0.32]$ \\
\hline \multicolumn{7}{|c|}{ Heterogeneity: $\mathrm{Tau}^{2}=0.01 ; \mathrm{Chi}^{2}=3.23, \mathrm{df}=3(\mathrm{P}=0.36) ; \mathrm{I}^{2}=6.98 \%$} \\
\hline \multicolumn{7}{|c|}{ Test for overall effect: $Z=0.05(P=0.96)$} \\
\hline \multicolumn{7}{|c|}{ 1.11.6 visual learning/memory } \\
\hline Kelly 2009 & 10 & 12 & $-0.4(0.434)$ & & $100 \%$ & $-0.44[-1.29,0.41]$ \\
\hline Subtotal $(95 \% \mathrm{Cl})$ & & & & & $100 \%$ & $-0.44[-1.29,0.41]$ \\
\hline \multicolumn{7}{|c|}{ Heterogeneity: Not applicable } \\
\hline \multicolumn{7}{|c|}{ Test for overall effect: $Z=1.01(P=0.31)$} \\
\hline Test for subgroup dif & $d f=1(P=0$. & $I^{2}=0 \%$ & & & & \\
\hline
\end{tabular}


Analysis 1.12. Comparison 1 SELECTIVE NORADRENALINE REUPTAKE INHIBITORS versus PLACEBO, Outcome 12 Quality of life: Average quality of life score (GQOLI-74, high=better) - short term (2 - 12 weeks).

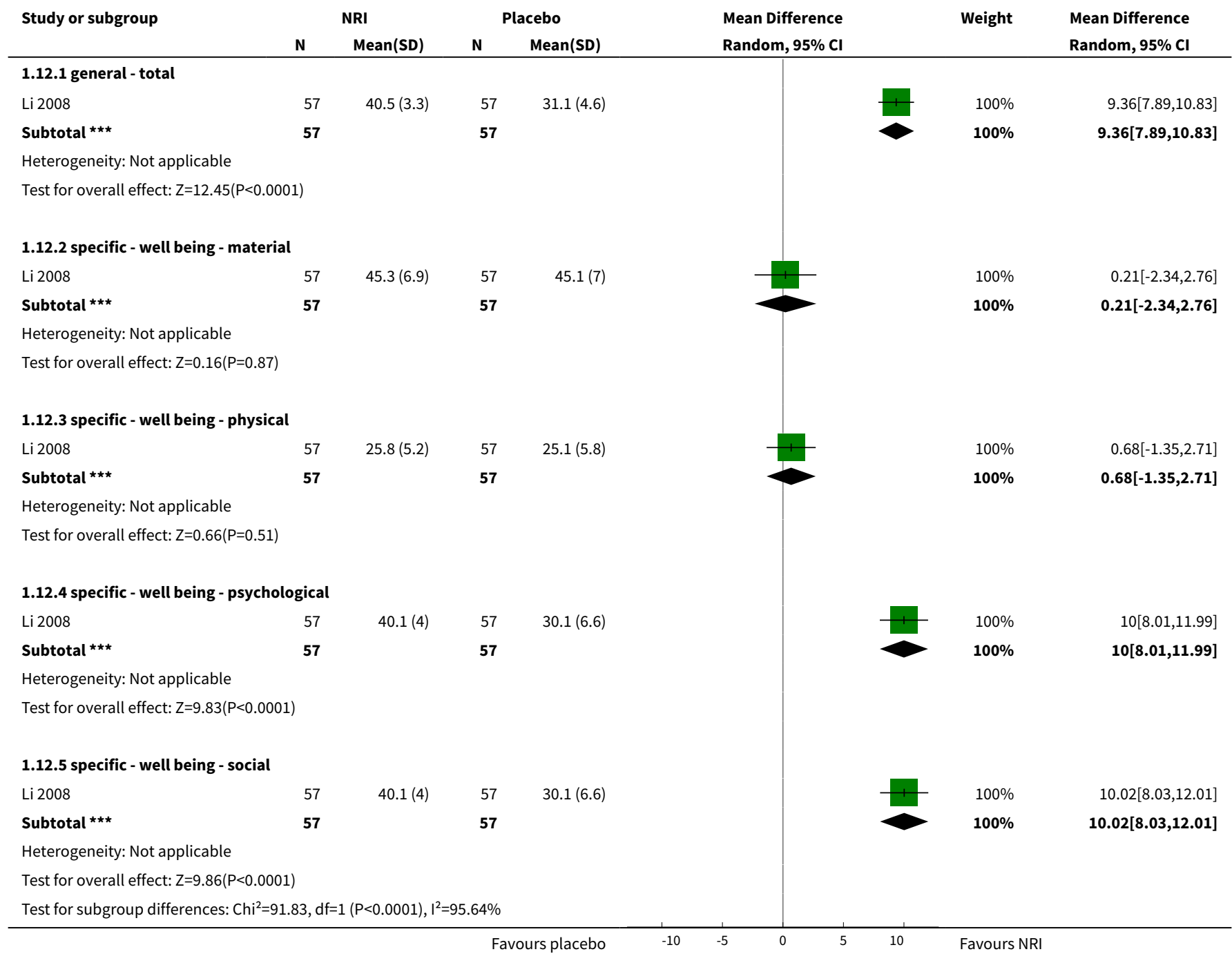

Analysis 1.13. Comparison 1 SELECTIVE NORADRENALINE REUPTAKE INHIBITORS versus PLACEBO, Outcome 13 Clinical global response: 1a. Average clinical global status score (CGI-S, high=worse) - short term (2 - 12 weeks).

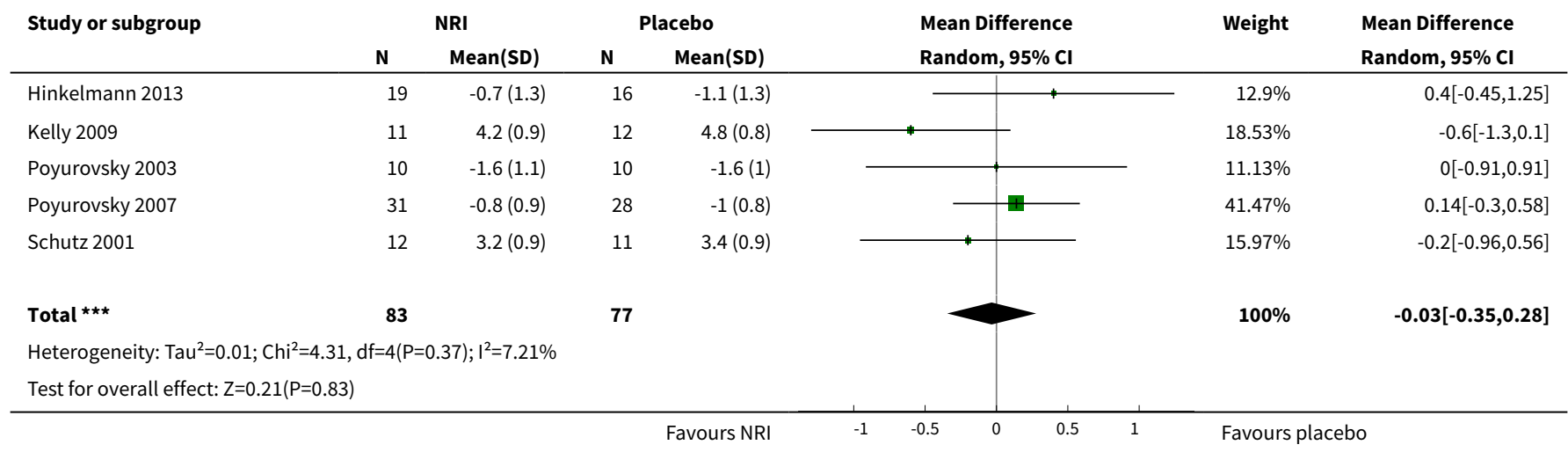


Analysis 1.14. Comparison 1 SELECTIVE NORADRENALINE REUPTAKE INHIBITORS versus PLACEBO, Outcome 14 Clinical global response: $1 \mathrm{~b}$. Average clinical global status score (various scales, high=worse) - short term ( 2 - 12 weeks) - skewed results.

Clinical global response: 1b. Average clinical global status score (various scales, high=worse) - short term (2 - 12 weeks) - skewed results

\begin{tabular}{|c|c|c|c|c|c|c|c|}
\hline Study & NRI & NRI mean & NRI SD & NRI N & Placebo mean & Placebo SD & Placebo N \\
\hline \multicolumn{8}{|c|}{$\begin{array}{c}\text { CGI- } \\
\text { I }\end{array}$} \\
\hline Schutz 2001 & Reboxetine & 3.4 & 1.35 & 12 & 3.07 & 1.22 & 11 \\
\hline \multicolumn{8}{|c|}{ CGI-S } \\
\hline Hinkelmann 2013 & Reboxetine & 4.2 & 1.5 & 19 & 3.6 & 1.3 & 16 \\
\hline Poyurovsky 2003 & Reboxetine & 3.1 & 1.3 & 10 & 3 & 1.1 & 10 \\
\hline
\end{tabular}

Analysis 1.15. Comparison 1 SELECTIVE NORADRENALINE REUPTAKE INHIBITORS versus PLACEBO, Outcome 15 Leaving the study early: 1a. Short term (2 - 12 weeks).

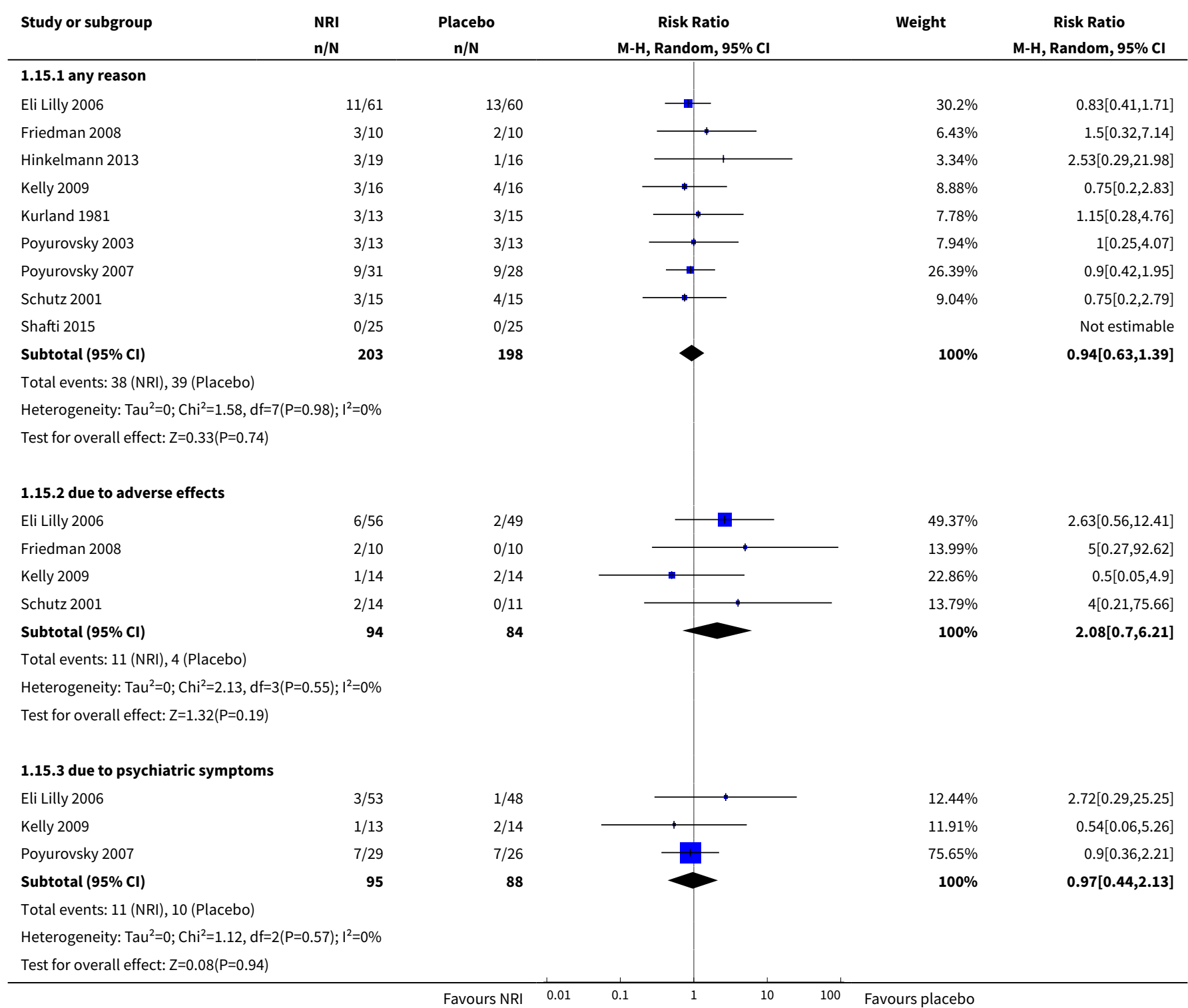




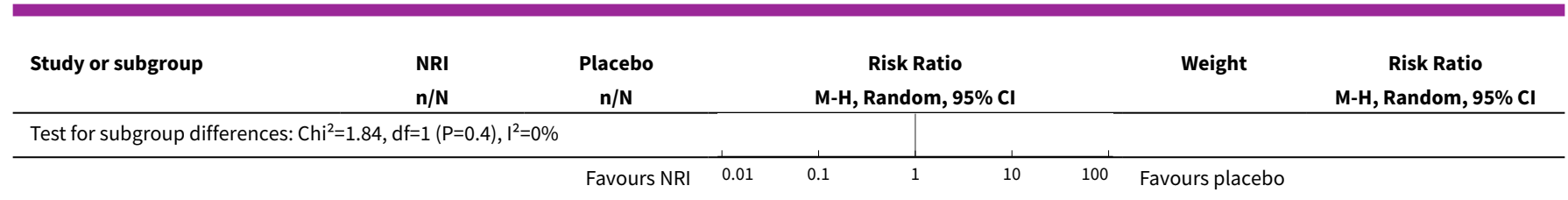

Analysis 1.16. Comparison 1 SELECTIVE NORADRENALINE REUPTAKE INHIBITORS versus PLACEBO, Outcome 16 Leaving the study early: 1b. Medium term (13 - 26 weeks).

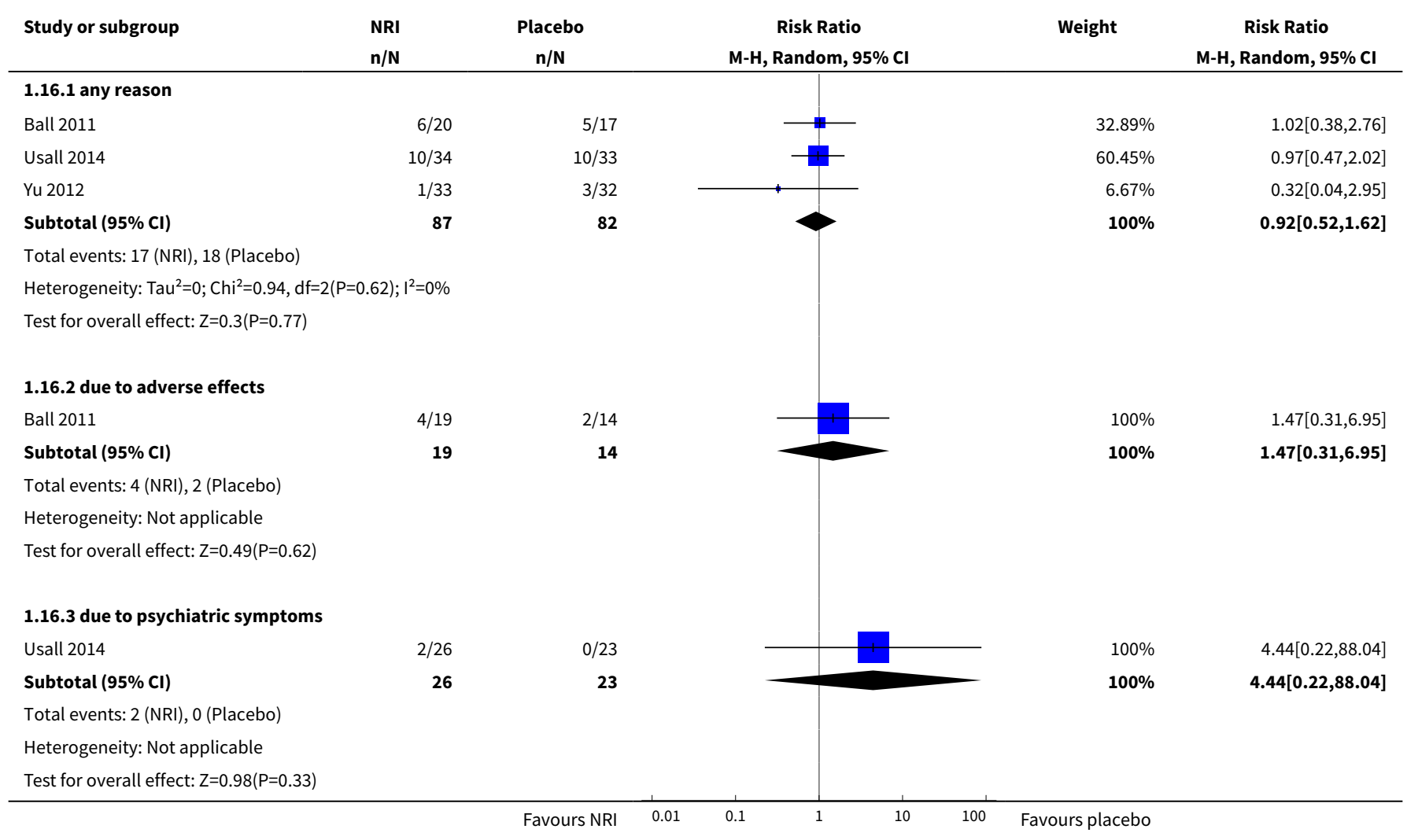

\section{Analysis 1.17. Comparison 1 SELECTIVE NORADRENALINE REUPTAKE INHIBITORS versus PLACEBO, Outcome 17 Adverse effects: 1. General - short term (binary, 2 - 12 weeks).}

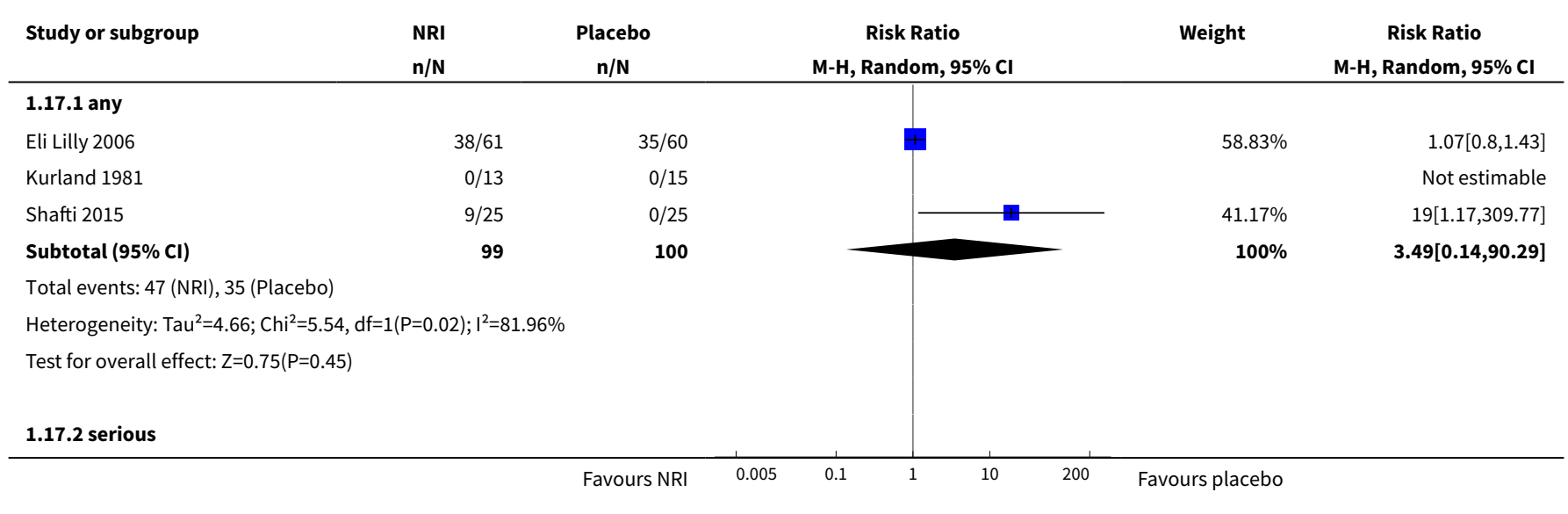




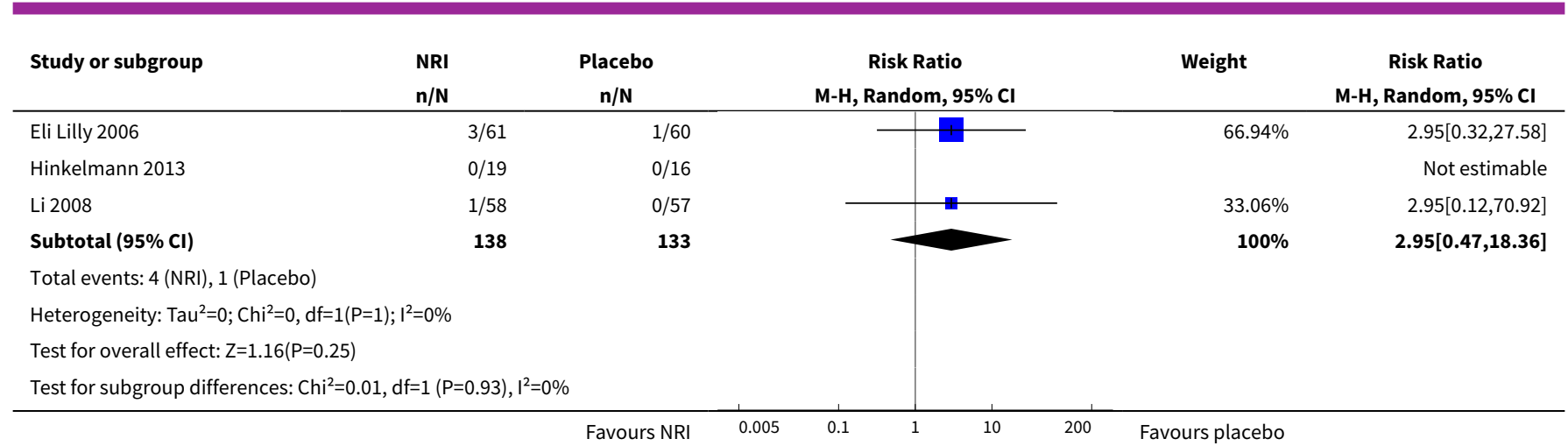

Analysis 1.18. Comparison 1 SELECTIVE NORADRENALINE REUPTAKE INHIBITORS versus PLACEBO, Outcome 18 Adverse effects: 2a.i. Specific - short term (binary, 2 - 12 weeks).

\begin{tabular}{|c|c|c|c|c|c|}
\hline Study or subgroup & & $\begin{array}{c}\text { Placebo } \\
n / N\end{array}$ & $\begin{array}{c}\text { Risk Ratio } \\
\text { M-H, Random, } 95 \% \mathrm{Cl}\end{array}$ & Weight & \multirow[t]{2}{*}{$\begin{array}{c}\text { Risk Ratio } \\
\text { M-H, Random, } 95 \% \text { CI }\end{array}$} \\
\hline \multicolumn{5}{|c|}{ 1.18.1 anticholinergic - constipation } & \\
\hline Kelly 2009 & $1 / 16$ & $3 / 16$ & $\longrightarrow$ & $27.79 \%$ & $0.33[0.04,2.87]$ \\
\hline Li 2008 & $4 / 57$ & $4 / 57$ & & $72.21 \%$ & $1[0.26,3.81]$ \\
\hline Subtotal $(95 \% \mathrm{Cl})$ & 73 & 73 & & $100 \%$ & $0.74[0.24,2.29]$ \\
\hline \multicolumn{6}{|c|}{ Total events: 5 (NRI), 7 (Placebo) } \\
\hline \multicolumn{6}{|c|}{ Test for overall effect: $Z=0.53(P=0.6)$} \\
\hline \multicolumn{6}{|c|}{ 1.18.2 anticholinergic - dry mouth } \\
\hline Kelly 2009 & $6 / 16$ & $1 / 16$ & & $20.4 \%$ & $6[0.81,44.35]$ \\
\hline Li 2008 & $12 / 57$ & $4 / 57$ & & $71.24 \%$ & $3[1.03,8.75]$ \\
\hline Schutz 2001 & $1 / 15$ & $0 / 15$ & & $8.36 \%$ & $3[0.13,68.26]$ \\
\hline \multicolumn{6}{|c|}{ Total events: 19 (NRI), 5 (Placebo) } \\
\hline \multicolumn{6}{|c|}{ Heterogeneity: $\operatorname{Tau}^{2}=0 ; \mathrm{Chi}^{2}=0.37, \mathrm{df}=2(\mathrm{P}=0.83) ; \mathrm{I}^{2}=0 \%$} \\
\hline \multicolumn{6}{|c|}{ Test for overall effect: $Z=2.69(P=0.01)$} \\
\hline \multicolumn{6}{|c|}{ 1.18.3 anticholinergic - impotence } \\
\hline Schutz 2001 & $1 / 15$ & $0 / 15$ & & $100 \%$ & $3[0.13,68.26]$ \\
\hline Subtotal $(95 \% \mathrm{Cl})$ & 15 & 15 & & $100 \%$ & $3[0.13,68.26]$ \\
\hline \multicolumn{6}{|c|}{ Total events: 1 (NRI), 0 (Placebo) } \\
\hline \multicolumn{6}{|c|}{ Heterogeneity: Not applicable } \\
\hline \multicolumn{6}{|c|}{ Test for overall effect: $Z=0.69(P=0.49)$} \\
\hline \multicolumn{6}{|c|}{ 1.18.4 cardiovascular - tachycardia } \\
\hline Li 2008 & $10 / 57$ & $3 / 57$ & & $100 \%$ & $3.33[0.97,11.48]$ \\
\hline \multicolumn{6}{|c|}{ Heterogeneity: Not applicable } \\
\hline \multicolumn{6}{|c|}{ Test for overall effect: $Z=1.91(P=0.06)$} \\
\hline \multicolumn{6}{|c|}{ 1.18.5 central nervious system - anorexia } \\
\hline Kelly 2009 & $0 / 16$ & $3 / 16$ & & $100 \%$ & $0.14[0.01,2.56]$ \\
\hline Subtotal (95\% CI) & 16 & 16 & & $100 \%$ & $0.14[0.01,2.56]$ \\
\hline
\end{tabular}




\begin{tabular}{ccccc} 
& & & \\
Study or subgroup & NRI & Placebo & Risk Ratio & Reight \\
& $\mathrm{n} / \mathrm{N}$ & $\mathrm{n} / \mathrm{N}$ & $\mathrm{M}-\mathrm{H}$, Random, $95 \% \mathrm{Cl}$ & $\mathrm{M}-\mathrm{H}, \mathrm{Random}, 95 \% \mathrm{Cl}$ \\
\hline
\end{tabular}

Total events: 0 (NRI), 3 (Placebo)

Heterogeneity: Not applicable

Test for overall effect: $Z=1.32(P=0.19)$

\subsection{6 central nervous system - dizziness}

\section{Kelly 2009}

Li 2008

Schutz 2001

Subtotal $(95 \% \mathrm{CI})$

Total events: 8 (NRI), 5 (Placebo)

Heterogeneity: $\mathrm{Tau}^{2}=0 ; \mathrm{Chi}^{2}=0.28, \mathrm{df}=2(\mathrm{P}=0.87) ; \mathrm{I}^{2}=0 \%$

Test for overall effect: $Z=0.79(P=0.43)$

\subsection{7 central nervous system - fatigue}

Kelly 2009

Schutz 2001

Subtotal $(95 \% \mathrm{Cl})$

31

Total events: 4 (NRI), 4 (Placebo)

Heterogeneity: $\mathrm{Tau}^{2}=1.21 ; \mathrm{Chi}^{2}=2, \mathrm{df}=1(\mathrm{P}=0.16) ; \mathrm{I}^{2}=50.12 \%$

Test for overall effect: $Z=0(P=1)$

\subsection{8 central nervous system - headache}

Eli Lilly 2006

$10 / 61$

$8 / 60$

Subtotal $(95 \% \mathrm{Cl})$

61

Total events: 10 (NRI), 8 (Placebo)

Heterogeneity: Not applicable

Test for overall effect: $\mathrm{Z}=0.47(\mathrm{P}=0.64)$

\subsection{9 central nervous system - insomnia}

Kelly 2009

Subtotal $(95 \% \mathrm{CI})$

Total events: 8 (NRI), 1 (Placebo)

Heterogeneity: $\mathrm{Tau}^{2}=0 ; \mathrm{Chi}^{2}=0.54, \mathrm{df}=1(\mathrm{P}=0.46) ; \mathrm{I}^{2}=0 \%$

Test for overall effect: $Z=1.78(P=0.07)$

\subsubsection{0 central nervous system - sedation}

$1 / 16 \quad 2 / 16$

Poyurovsky 2003

Subtotal $(95 \% \mathrm{Cl})$

29

$2 / 16$

$5 / 13$

Total events: 5 (NRI), 7 (Placebo)

Heterogeneity: $\mathrm{Tau}^{2}=0 ; \mathrm{Chi}^{2}=0.14, \mathrm{df}=1(\mathrm{P}=0.71) ; \mathrm{I}^{2}=0 \%$

Test for overall effect: $\mathrm{Z}=0.62(\mathrm{P}=0.53)$

\subsubsection{1 extrapyramidal - acute dystonia}

Schutz 2001

Subtotal $(95 \% \mathrm{Cl})$

15

$1 / 16$

$0 / 57$

73

60

Total events: 1 (NRI), 1 (Placebo)

Heterogeneity: Not applicable

Test for overall effect: Not applicable

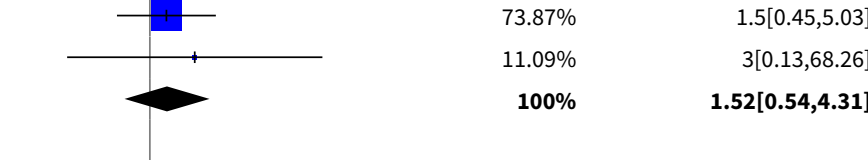

$49.91 \%$

$3[0.35,25.87]$

$50.09 \%$

$0.33[0.04,2.85]$

$100 \%$

$1[0.12,8.6]$

$100 \%$

$1.23[0.52,2.9]$

$100 \%$

$1.23[0.52,2.9]$

$63.99 \%$

$3[0.35,25.87]$

$36.01 \%$

$11[0.62,194.41]$

$100 \%$

$4.79[0.85,26.84]$

$17.72 \%$

$0.5[0.05,4.98]$

$82.28 \%$

$0.8[0.28,2.32]$

$100 \%$

$0.74[0.28,1.94]$

$100 \%$

$1[0.07,14.55]$

$100 \%$ 


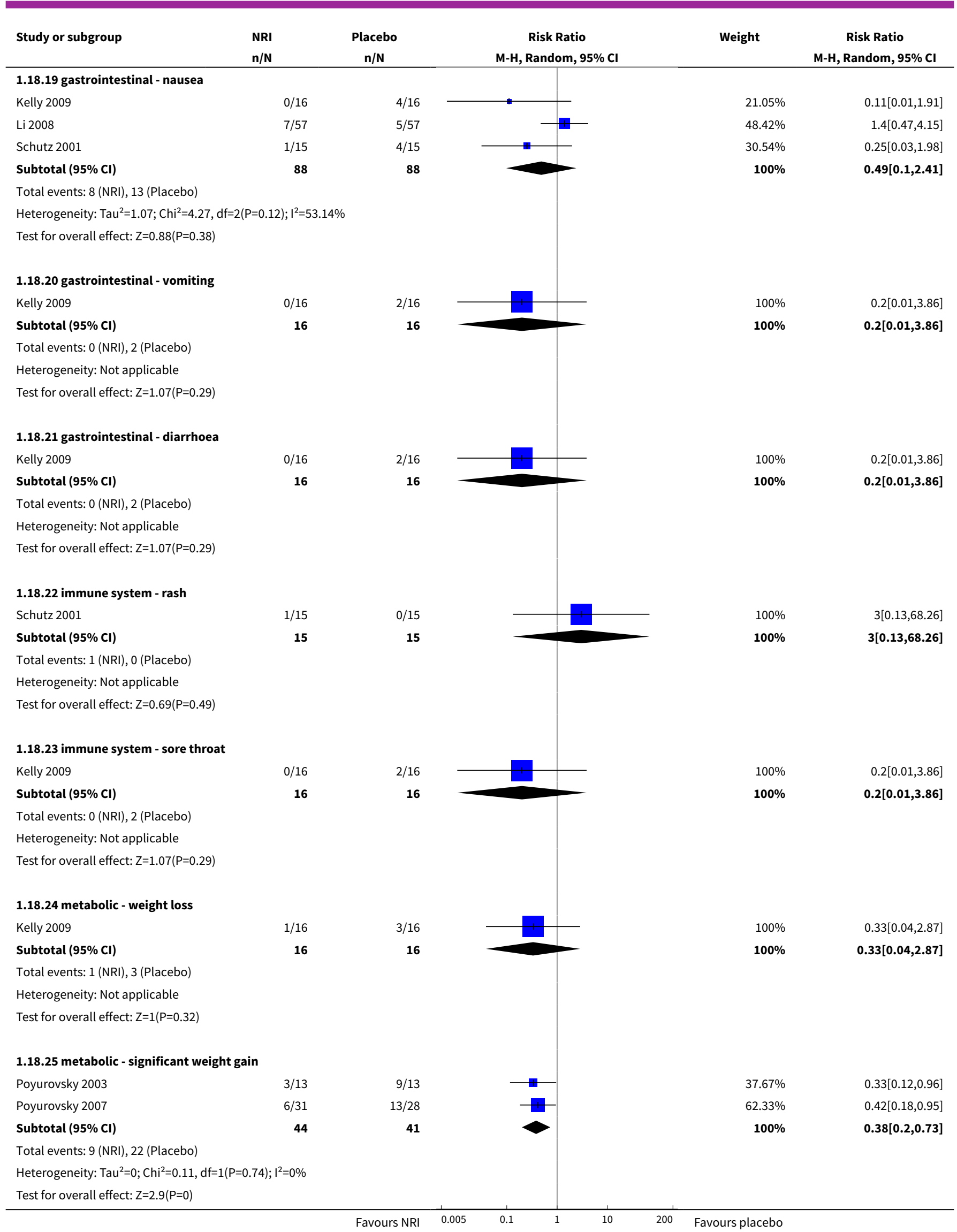


Analysis 1.19. Comparison 1 SELECTIVE NORADRENALINE REUPTAKE INHIBITORS versus PLACEBO, Outcome 19 Adverse effects: 2a.ii. Specific - extrapyramidal - average change score (continuous, various scales, high=worse) - short term ( 2 - 12 weeks).

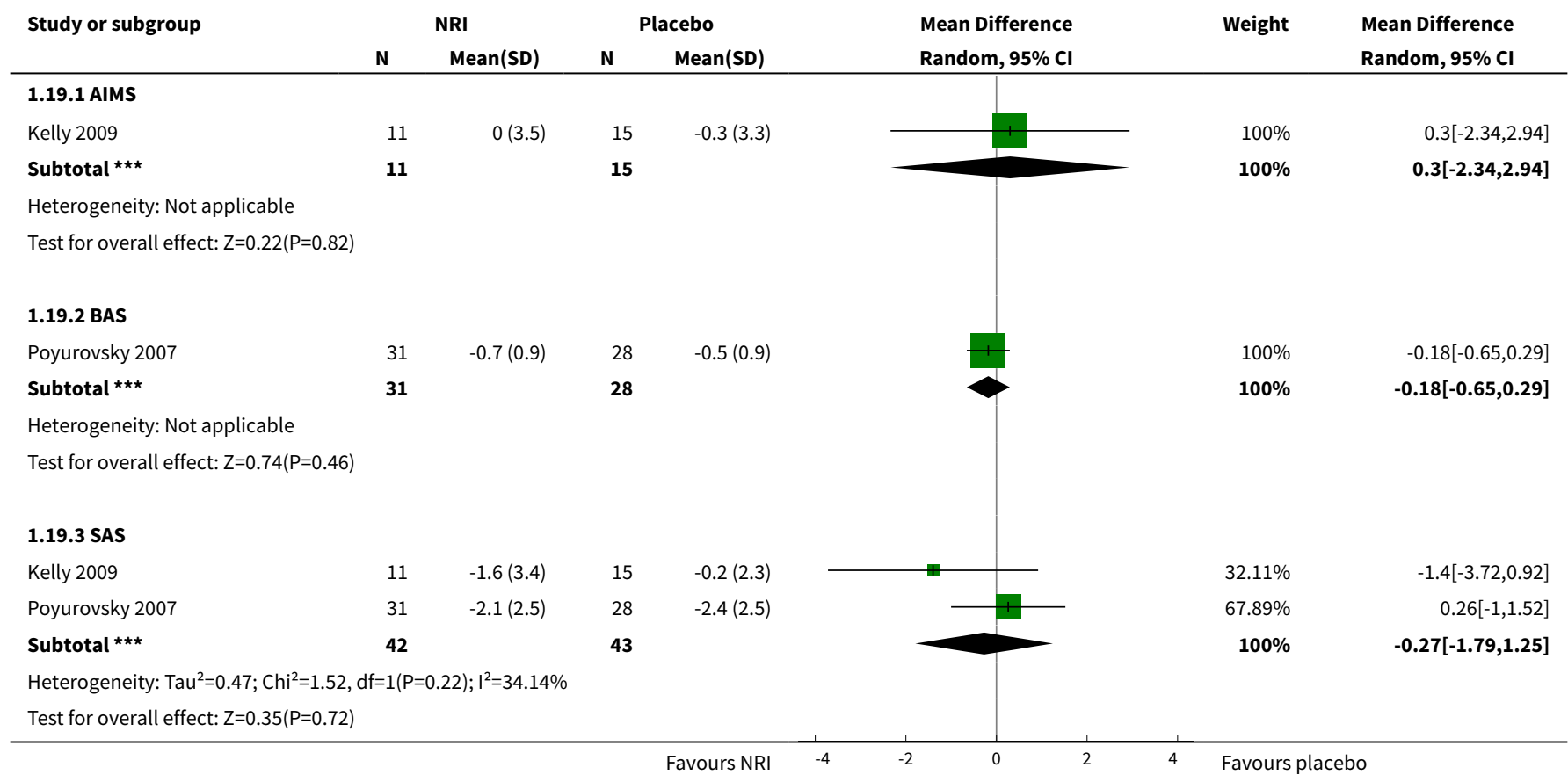

Analysis 1.20. Comparison 1 SELECTIVE NORADRENALINE REUPTAKE INHIBITORS versus PLACEBO, Outcome 20 Adverse effects: 2 a.iii. Specific - extrapyramidal - average change score (continuous, various scales, high=worse) - short term (2 - 12 weeks) - skewed results.

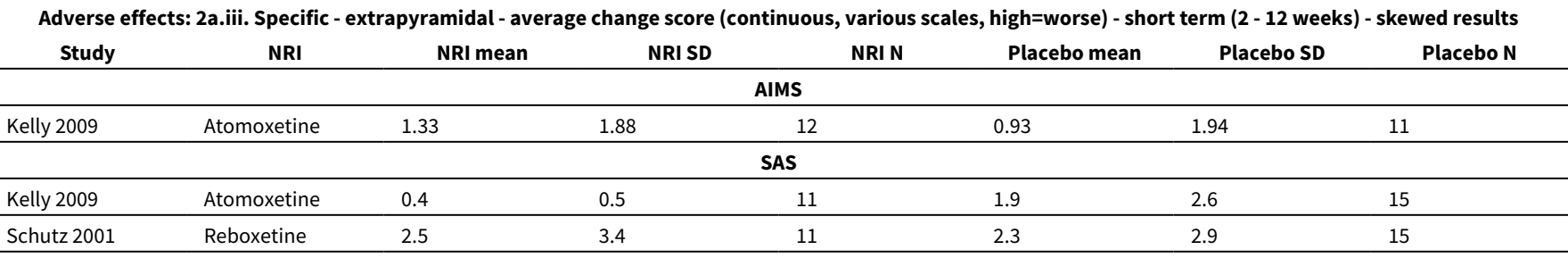

Analysis 1.21. Comparison 1 SELECTIVE NORADRENALINE REUPTAKE INHIBITORS versus PLACEBO, Outcome 21 Adverse effects: 2 b.i. Specific - medium term (binary, 13 - 26 weeks).

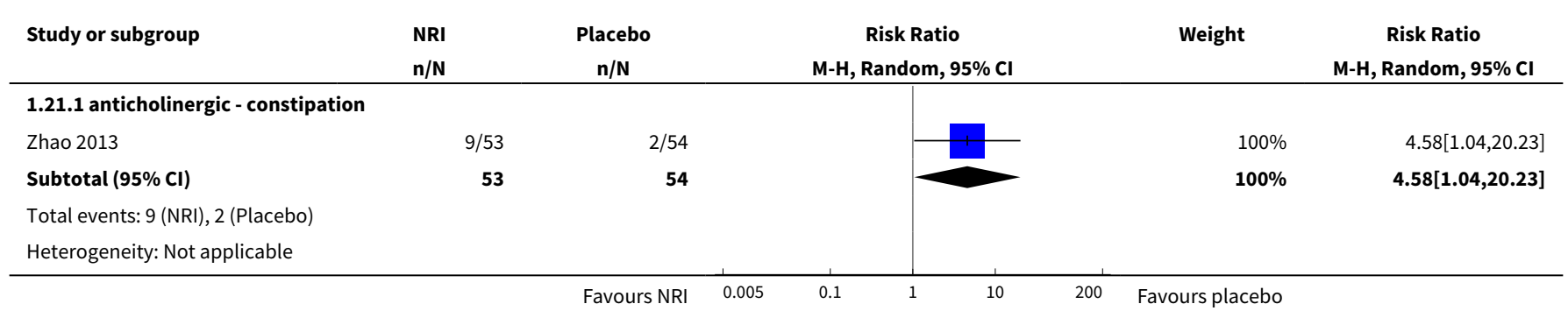




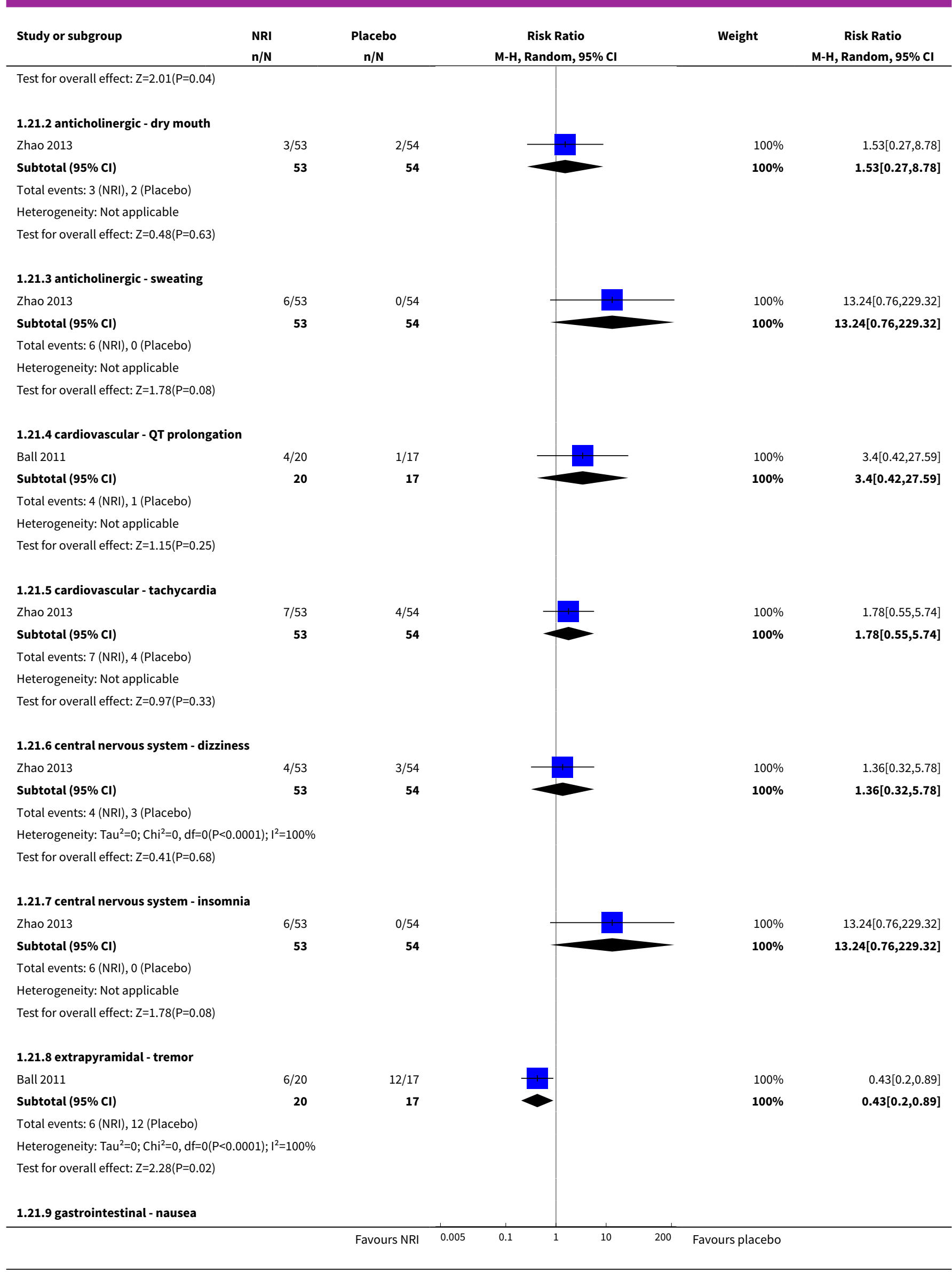




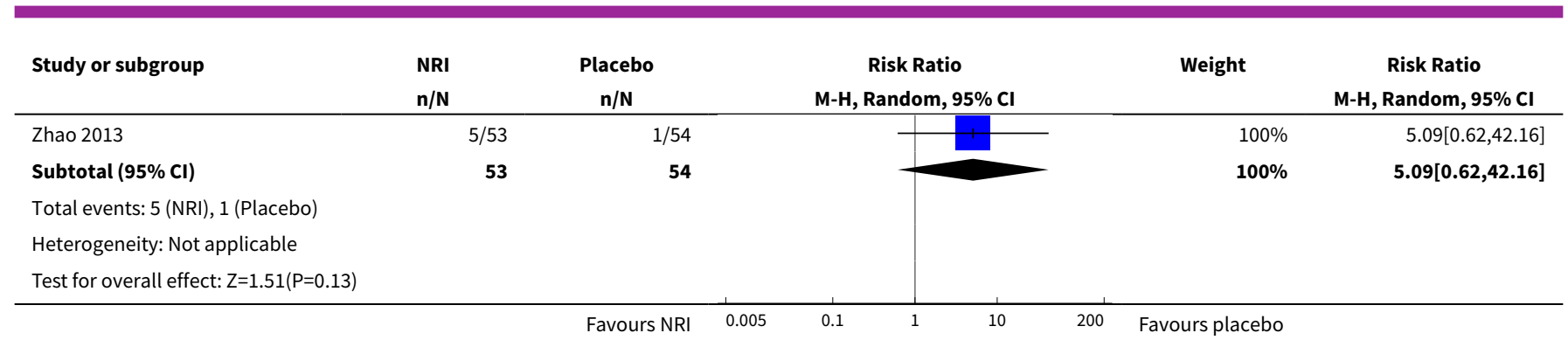

Analysis 1.22. Comparison 1 SELECTIVE NORADRENALINE REUPTAKE INHIBITORS versus PLACEBO, Outcome 22 Adverse effects: 2 a.iv. Specific - metabolic - average weight gain (continuous, increase in kg).

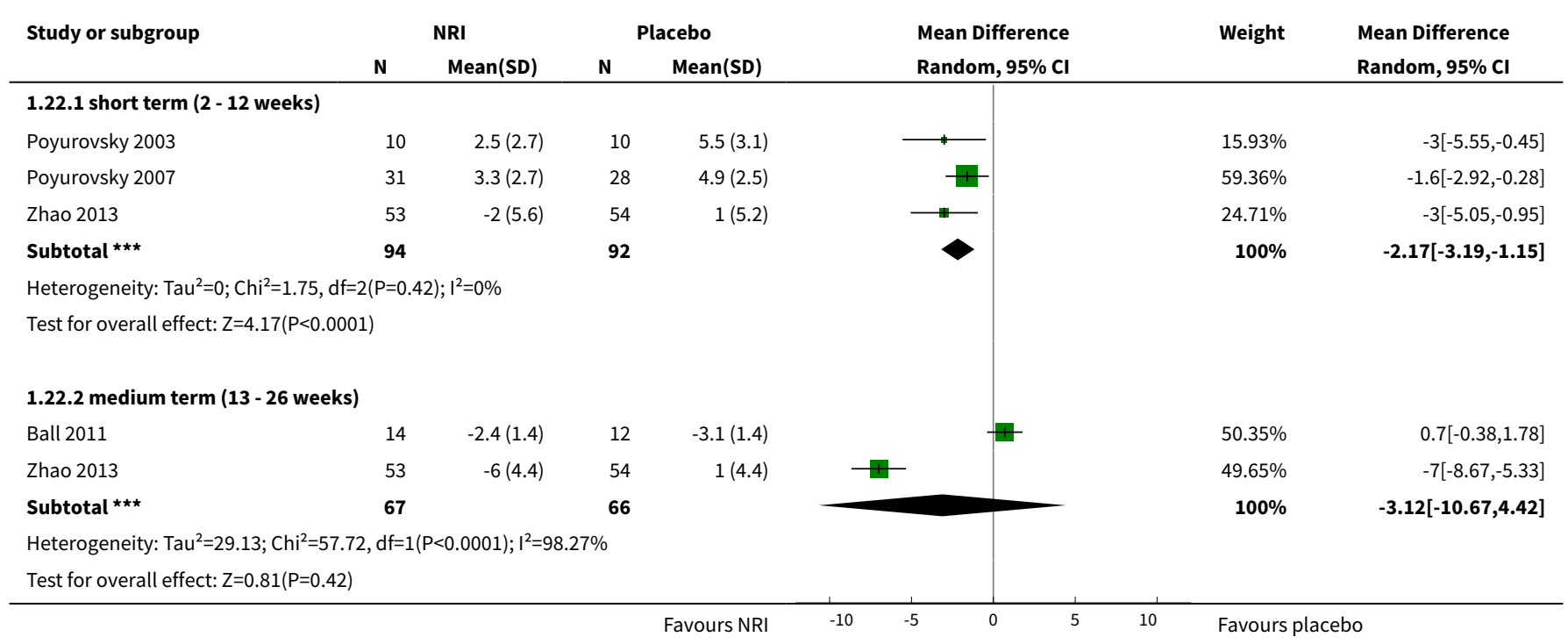
Analysis 1.23. Comparison 1 SELECTIVE NORADRENALINE REUPTAKE INHIBITORS versus PLACEBO, Outcome 23
Social or general functioning: Average social functioning score (various subscales) - short-term ( 2 - 12 weeks).

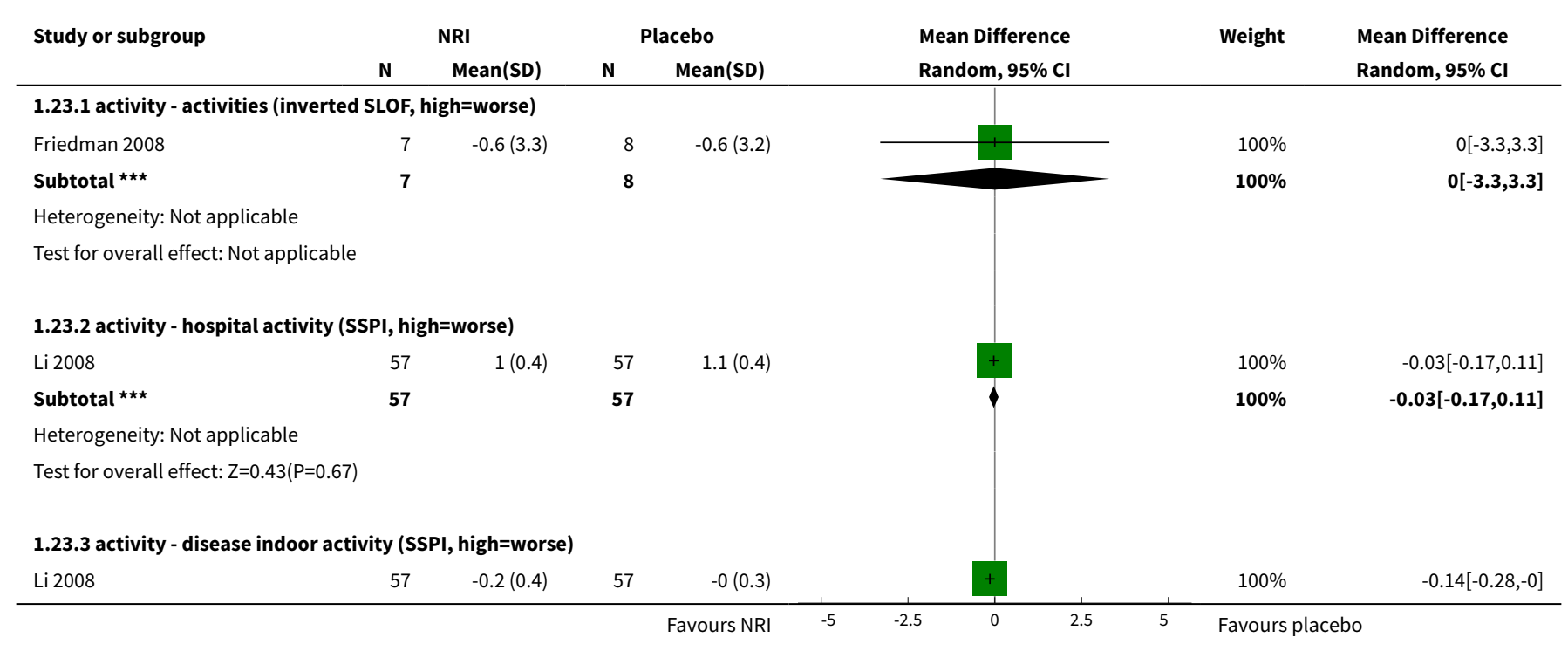




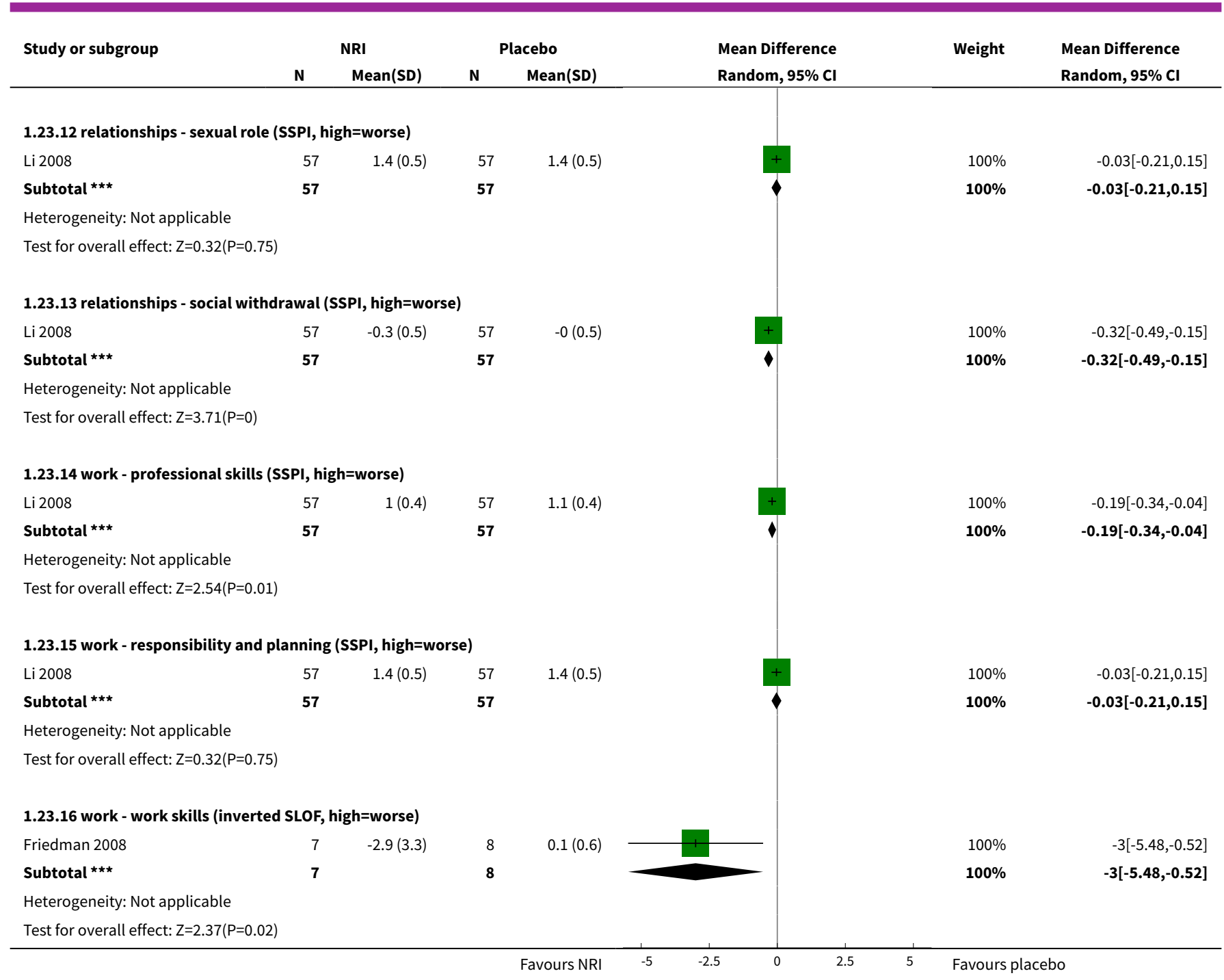

\section{Comparison 2. SELECTIVE NORADRENALINE REUPTAKE INHIBITORS verSUS ACTIVE CONTROL}

\begin{tabular}{|c|c|c|c|c|}
\hline Outcome or subgroup title & No. of studies & $\begin{array}{l}\text { No. of partici- } \\
\text { pants }\end{array}$ & Statistical method & Effect size \\
\hline $\begin{array}{l}1 \text { Mental state: 1a. General: Average gener- } \\
\text { al/overall symptoms score (PANSS general, } \\
\text { high=worse) - short term ( } 2 \text { - } 12 \text { weeks) }\end{array}$ & 1 & 35 & $\begin{array}{l}\text { Mean Difference (IV, } \\
\text { Random, } 95 \% \mathrm{CI} \text { ) }\end{array}$ & $2.90[-2.85,8.65]$ \\
\hline $\begin{array}{l}2 \text { Mental state: } 1 \text { b. General: Average gener- } \\
\text { al/overall symptoms score (various scales, } \\
\text { high=worse) - medium term ( } 13 \text { - } 26 \text { weeks) }\end{array}$ & 1 & & $\begin{array}{l}\text { Mean Difference (IV, } \\
\text { Random, } 95 \% \mathrm{CI} \text { ) }\end{array}$ & Subtotals only \\
\hline 2.1 PANSS total & 1 & 41 & $\begin{array}{l}\text { Mean Difference (IV, } \\
\text { Random, } 95 \% \mathrm{CI} \text { ) }\end{array}$ & $\begin{array}{l}1.62[-6.89 \\
10.13]\end{array}$ \\
\hline 2.2 PANSS general & 1 & 41 & $\begin{array}{l}\text { Mean Difference (IV, } \\
\text { Random, } 95 \% \mathrm{CI} \text { ) }\end{array}$ & $0.62[-2.00,5.24]$ \\
\hline
\end{tabular}




\begin{tabular}{|c|c|c|c|c|}
\hline Outcome or subgroup title & No. of studies & $\begin{array}{l}\text { No. of partici- } \\
\text { pants }\end{array}$ & Statistical method & Effect size \\
\hline $\begin{array}{l}3 \text { Mental state: } 2 \text { a. Specific: Average symp- } \\
\text { toms score (various scales, high=worse) - } \\
\text { short term ( } 2 \text { - } 12 \text { weeks) }\end{array}$ & 1 & & $\begin{array}{l}\text { Mean Difference (IV, } \\
\text { Fixed, } 95 \% \mathrm{CI} \text { ) }\end{array}$ & Subtotals only \\
\hline $3.1 \operatorname{mood}(\mathrm{HRSD})$ & 1 & 35 & $\begin{array}{l}\text { Mean Difference (IV, } \\
\text { Fixed, } 95 \% \mathrm{CI} \text { ) }\end{array}$ & $3.00[-1.24,7.24]$ \\
\hline 3.2 negative (PANSS negative) & 1 & 35 & $\begin{array}{l}\text { Mean Difference (IV, } \\
\text { Fixed, } 95 \% \mathrm{CI} \text { ) }\end{array}$ & $1.5[-4.34,7.34]$ \\
\hline 3.3 positive (PANSS positive) & 1 & 35 & $\begin{array}{l}\text { Mean Difference (IV, } \\
\text { Fixed, } 95 \% \mathrm{CI} \text { ) }\end{array}$ & $1.7[-1.52,4.92]$ \\
\hline $\begin{array}{l}4 \text { Mental state: } 2 \text { b. Specific: Average symp- } \\
\text { toms score (various scales, high=worse) - } \\
\text { medium term ( } 13 \text { - } 26 \text { weeks) }\end{array}$ & 1 & & $\begin{array}{l}\text { Mean Difference (IV, } \\
\text { Random, } 95 \% \mathrm{CI} \text { ) }\end{array}$ & Subtotals only \\
\hline 4.1 negative (PANSS negative) & 1 & 41 & $\begin{array}{l}\text { Mean Difference (IV, } \\
\text { Random, } 95 \% \mathrm{CI} \text { ) }\end{array}$ & $0.26[-3.66,4.18]$ \\
\hline 4.2 negative (SANS) & 1 & 41 & $\begin{array}{l}\text { Mean Difference (IV, } \\
\text { Random, } 95 \% \mathrm{CI} \text { ) }\end{array}$ & $\begin{array}{l}-0.27[-12.69 \\
12.15]\end{array}$ \\
\hline 4.3 positive (PANSS positive) & 1 & 41 & $\begin{array}{l}\text { Mean Difference (IV, } \\
\text { Random, } 95 \% \mathrm{CI} \text { ) }\end{array}$ & $0.74[-1.86,3.34]$ \\
\hline $\begin{array}{l}5 \text { Mental state: } 3 a \text {. General and specific: Av- } \\
\text { erage score (various scales, high=worse) - } \\
\text { skewed results - short term ( } 2-12 \text { weeks) }\end{array}$ & & & Other data & No numeric data \\
\hline
\end{tabular}

\begin{tabular}{|c|c|c|}
\hline $\begin{array}{l}5.1 \text { general - overall symptoms (PANSS gen- } \\
\text { eral) }\end{array}$ & Other data & No numeric data \\
\hline 5.2 specific - mood (HRSD) & Other data & No numeric data \\
\hline $\begin{array}{l}5.3 \text { specific - negative symptoms (PANSS } \\
\text { negative) }\end{array}$ & Other data & No numeric data \\
\hline $\begin{array}{l}5.4 \text { specific - positive symptoms (PANSS pos- } \\
\text { itive) }\end{array}$ & Other data & No numeric data \\
\hline $\begin{array}{l}6 \text { Mental state: } 3 b \text {. General and specific: Av- } \\
\text { erage score (various scales, high=worse) } \\
\text { - skewed results - medium term (13 - } 26 \\
\text { weeks) }\end{array}$ & Other data & No numeric data \\
\hline $\begin{array}{l}6.1 \text { general - overall symptoms (PANSS gen- } \\
\text { eral) }\end{array}$ & Other data & No numeric data \\
\hline 6.2 general - overall symptoms (PANSS total) & Other data & No numeric data \\
\hline $\begin{array}{l}6.3 \text { specific - negative symptoms (PANSS } \\
\text { negative) }\end{array}$ & Other data & No numeric data \\
\hline 6.4 specific - negative symptoms (SANS) & Other data & No numeric data \\
\hline
\end{tabular}




\begin{tabular}{|c|c|c|c|c|}
\hline Outcome or subgroup title & No. of studies & $\begin{array}{l}\text { No. of partici- } \\
\text { pants }\end{array}$ & Statistical method & Effect size \\
\hline $\begin{array}{l}6.5 \text { specific - positive symptoms (PANSS pos- } \\
\text { itive) }\end{array}$ & & & Other data & No numeric data \\
\hline $\begin{array}{l}7 \text { Clinical global response: Average clinical } \\
\text { global status score (CGI-S, high=worse) - } \\
\text { short term ( } 2 \text { - } 12 \text { weeks) }\end{array}$ & 1 & 35 & $\begin{array}{l}\text { Mean Difference (IV, } \\
\text { Random, } 95 \% \mathrm{CI} \text { ) }\end{array}$ & $0.0[-0.89,0.89]$ \\
\hline $\begin{array}{l}8 \text { Leaving the study early: } 1 \text { a. Short term ( } 2 \text { - } \\
12 \text { weeks) }\end{array}$ & 1 & & $\begin{array}{l}\text { Risk Ratio (M-H, Ran- } \\
\text { dom, } 95 \% \mathrm{Cl} \text { ) }\end{array}$ & Subtotals only \\
\hline 8.1 any reason & 1 & 35 & $\begin{array}{l}\text { Risk Ratio (M-H, Ran- } \\
\text { dom, } 95 \% \mathrm{Cl})\end{array}$ & $0.63[0.17,2.41]$ \\
\hline $\begin{array}{l}9 \text { Leaving the study early: } 1 \text { b. Medium term } \\
\text { (13 - } 26 \text { weeks) }\end{array}$ & 1 & & $\begin{array}{l}\text { Risk Ratio (M-H, Ran- } \\
\text { dom, } 95 \% \mathrm{Cl})\end{array}$ & Subtotals only \\
\hline 9.1 any reason & 1 & 57 & $\begin{array}{l}\text { Risk Ratio (M-H, Ran- } \\
\text { dom, } 95 \% \mathrm{Cl} \text { ) }\end{array}$ & $1.13[0.48,2.67]$ \\
\hline 9.2 due to psychiatric symptoms & 1 & 49 & $\begin{array}{l}\text { Risk Ratio (M-H, Ran- } \\
\text { dom, } 95 \% \mathrm{Cl})\end{array}$ & $1.77[0.17,18.26]$ \\
\hline
\end{tabular}

Analysis 2.1. Comparison 2 SELECTIVE NORADRENALINE REUPTAKE INHIBITORS versus ACTIVE CONTROL, Outcome 1 Mental state: 1a. General: Average general/ overall symptoms score (PANSS general, high=worse) - short term (2 - 12 weeks).

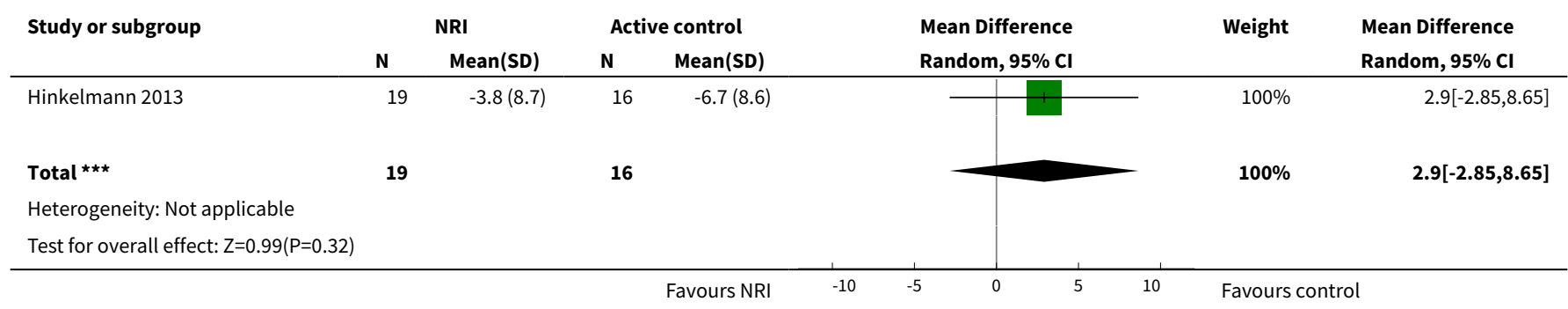

Analysis 2.2. Comparison 2 SELECTIVE NORADRENALINE REUPTAKE INHIBITORS versus ACTIVE CONTROL, Outcome 2 Mental state: 1b. General: Average general/ overall symptoms score (various scales, high=worse) - medium term (13 - 26 weeks).

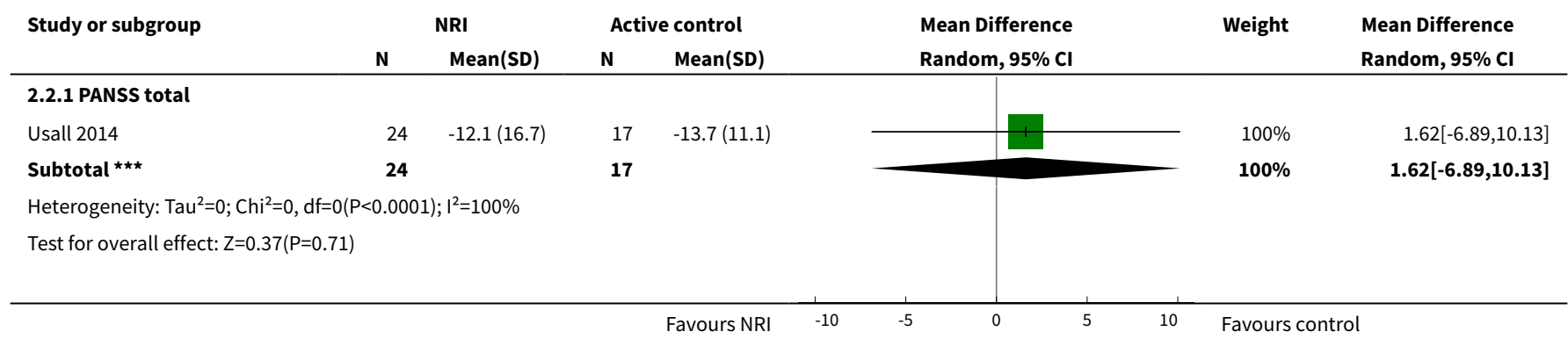




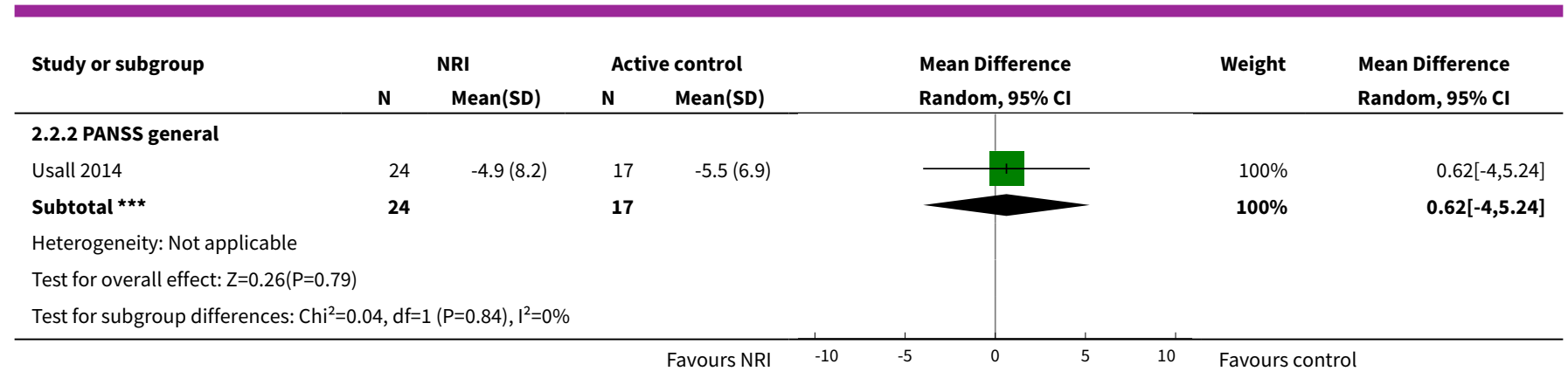

Analysis 2.3. Comparison 2 SELECTIVE NORADRENALINE REUPTAKE INHIBITORS versus ACTIVE CONTROL, Outcome 3 Mental state: 2a. Specific: Average symptoms score (various scales, high=worse) - short term (2 - 12 weeks).

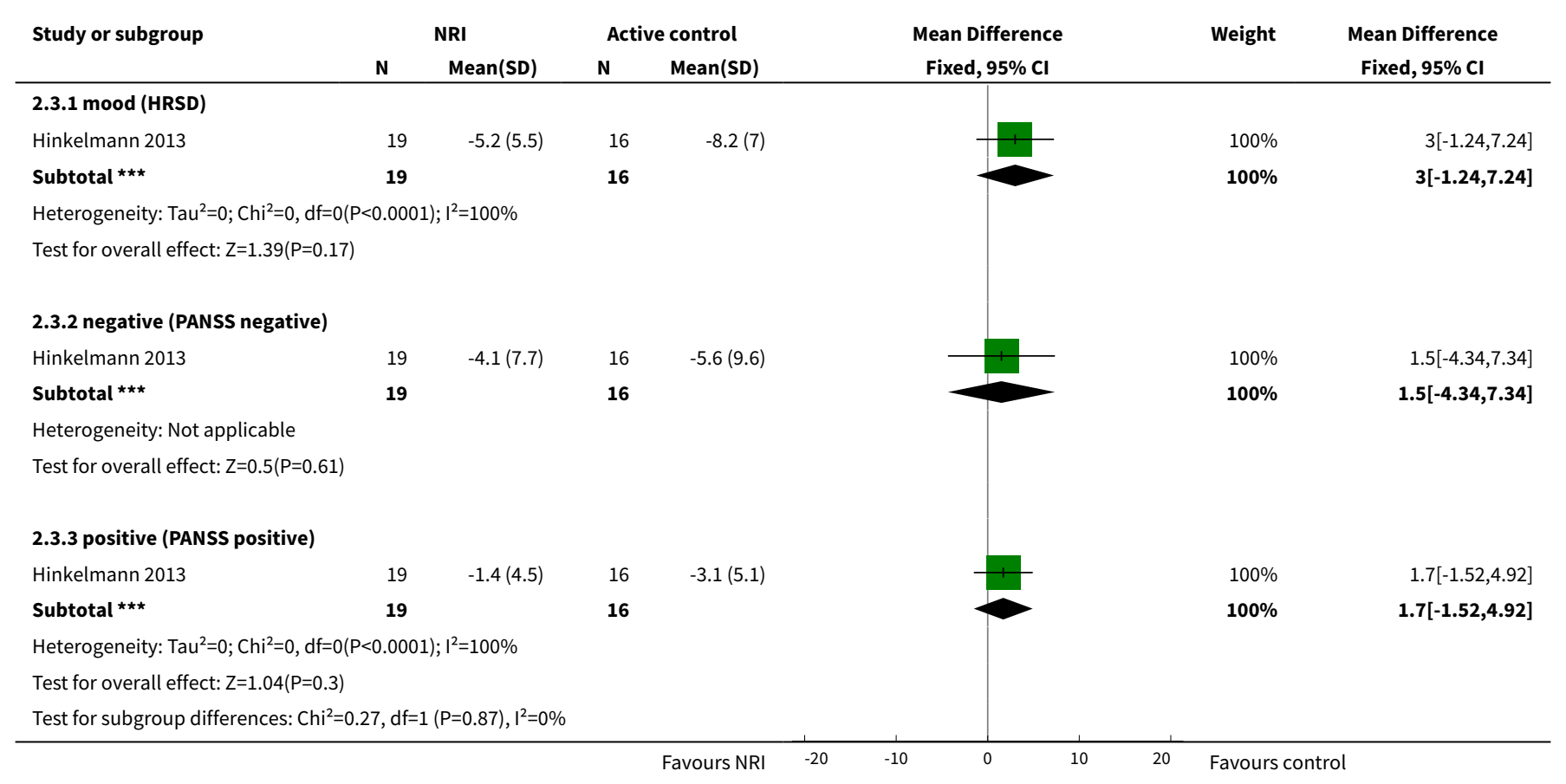

Analysis 2.4. Comparison 2 SELECTIVE NORADRENALINE REUPTAKE INHIBITORS versus ACTIVE CONTROL, OutCome 4 Mental state: 2b. Specific: Average symptoms score (various scales, high=worse) - medium term (13 - 26 weeks).

\begin{tabular}{|c|c|c|c|c|c|c|c|}
\hline \multirow[t]{2}{*}{ Study or subgroup } & \multicolumn{2}{|r|}{ NRI } & \multicolumn{2}{|c|}{ Active control } & \multirow{2}{*}{$\begin{array}{l}\text { Mean Difference } \\
\text { Random, } 95 \% \mathrm{Cl}\end{array}$} & \multirow[t]{2}{*}{ Weight } & \multirow{2}{*}{$\begin{array}{l}\text { Mean Difference } \\
\text { Random, 95\% Cl }\end{array}$} \\
\hline & $\mathbf{N}$ & Mean(SD) & $\mathbf{N}$ & Mean(SD) & & & \\
\hline \multicolumn{8}{|c|}{ 2.4.1 negative (PANSS negative) } \\
\hline Usall 2014 & 24 & $-5.8(7.7)$ & 17 & $-6.1(5.1)$ & & $100 \%$ & $0.26[-3.66,4.18]$ \\
\hline Subtotal $\star \star \star$ & 24 & & 17 & & & $100 \%$ & $0.26[-3.66,4.18]$ \\
\hline \multicolumn{8}{|c|}{ Heterogeneity: Not applicable } \\
\hline \multicolumn{8}{|c|}{ Test for overall effect: $Z=0.13(P=0.9)$} \\
\hline \multicolumn{8}{|c|}{ 2.4.2 negative (SANS) } \\
\hline Usall 2014 & 24 & $-14.8(23.6)$ & 17 & $-14.5(17)$ & & $100 \%$ & $-0.27[-12.69,12.15]$ \\
\hline \multirow[t]{2}{*}{ Subtotal $\star \star \star$} & 24 & & 17 & & & $100 \%$ & $-0.27[-12.69,12.15]$ \\
\hline & & & & Favours NRI & $-10 \quad-5$ & Favours & \\
\hline
\end{tabular}




\begin{tabular}{|c|c|c|c|c|c|c|c|}
\hline \multirow[t]{2}{*}{ Study or subgroup } & \multicolumn{2}{|c|}{ NRI } & \multicolumn{2}{|c|}{ Active control } & \multirow{2}{*}{$\begin{array}{l}\text { Mean Difference } \\
\text { Random, } 95 \% \mathrm{Cl} \\
\end{array}$} & \multirow[t]{2}{*}{ Weight } & \multirow{2}{*}{$\begin{array}{l}\text { Mean Difference } \\
\text { Random, } 95 \% \mathrm{Cl}\end{array}$} \\
\hline & $\mathbf{N}$ & $\operatorname{Mean}(S D)$ & $\mathbf{N}$ & Mean(SD) & & & \\
\hline \multicolumn{8}{|l|}{ Heterogeneity: Not applicable } \\
\hline \multicolumn{8}{|l|}{ Test for overall effect: $Z=0.04(P=0.97)$} \\
\hline \multicolumn{8}{|l|}{ 2.4.3 positive (PANSS positive) } \\
\hline Subtotal $\star \star \star$ & 24 & & 17 & & & $100 \%$ & $0.74[-1.86,3.34]$ \\
\hline \multicolumn{8}{|l|}{ Heterogeneity: Not applicable } \\
\hline \multicolumn{8}{|l|}{ Test for overall effect: $Z=0.56(P=0.58)$} \\
\hline & & & & Favours NRI & $-10 \quad-5$ & Favours C & \\
\hline
\end{tabular}

Analysis 2.5. Comparison 2 SELECTIVE NORADRENALINE REUPTAKE INHIBITORS versus ACTIVE CONTROL, Outcome 5 Mental state: 3a. General and specific: Average score (various scales, high=worse) - skewed results - short term (2 - 12 weeks).

\begin{tabular}{|c|c|c|c|c|c|c|c|c|}
\hline \multicolumn{9}{|c|}{ Mental state: 3a. General and specific: Average score (various scales, high=worse) - skewed results - short term (2 - 12 weeks) } \\
\hline Study & NRI & NRI mean & NRI SD & NRI N & Active control & Control mean & Control SD & Control N \\
\hline \multicolumn{9}{|c|}{ general - overall symptoms (PANSS general) } \\
\hline $\begin{array}{l}\text { Hinkelmann } \\
2013\end{array}$ & Reboxetine & 28.7 & 9.4 & 19 & Citalopram & 26.5 & 6.2 & 16 \\
\hline \multicolumn{9}{|c|}{ specific - mood (HRSD) } \\
\hline $\begin{array}{l}\text { Hinkelmann } \\
2013\end{array}$ & Reboxetine & 8.3 & 6.1 & 19 & Citalopram & 9.6 & 5.8 & 16 \\
\hline \multicolumn{9}{|c|}{ specific - negative symptoms (PANSS negative) } \\
\hline $\begin{array}{l}\text { Hinkelmann } \\
2013\end{array}$ & Reboxetine & 21.5 & 8.6 & 19 & Citalopram & 19.1 & 8.8 & 16 \\
\hline \multicolumn{9}{|c|}{ specific - positive symptoms (PANSS positive) } \\
\hline $\begin{array}{l}\text { Hinkelmann } \\
2013\end{array}$ & Reboxetine & 10.6 & 4.6 & 19 & Citalopram & 11.1 & 4.8 & 16 \\
\hline
\end{tabular}

Analysis 2.6. Comparison 2 SELECTIVE NORADRENALINE REUPTAKE INHIBITORS versus ACTIVE CONTROL, Outcome 6 Mental state: $3 \mathrm{~b}$. General and specific: Average score (various scales, high=worse) - skewed results - medium term (13 - 26 weeks).

\begin{tabular}{|c|c|c|c|c|c|c|c|c|}
\hline \multicolumn{9}{|c|}{ Mental state: 3b. General and specific: Average score (various scales, high=worse) - skewed results - medium term (13 - 26 weeks) } \\
\hline Study & NRI & NRI mean & NRI SD & NRI N & Active control & Control mean & Control SD & Control N \\
\hline \multicolumn{9}{|c|}{ general - overall symptoms (PANSS general) } \\
\hline Usall 2014 & Reboxetine & 28.83 & 8.1 & 24 & Citalopram & 28.88 & 5.32 & 17 \\
\hline \multicolumn{9}{|c|}{ general - overall symptoms (PANSS total) } \\
\hline Usall 2014 & Reboxetine & 59.96 & 17.89 & 24 & Citalopram & 59.41 & 11.72 & 17 \\
\hline \multicolumn{9}{|c|}{ specific - negative symptoms (PANSS negative) } \\
\hline Usall 2014 & Reboxetine & 19.67 & 8.41 & 24 & Citalopram & 19.82 & 4.99 & 17 \\
\hline \multicolumn{9}{|c|}{ specific - negative symptoms (SANS) } \\
\hline Usall 2014 & Reboxetine & 46.75 & 26.12 & 24 & Citalopram & 46.63 & 15.84 & 17 \\
\hline \multicolumn{9}{|c|}{ specific - positive symptoms (PANSS positive) } \\
\hline Usall 2014 & Reboxetine & 11.46 & 4.14 & 24 & Citalopram & 10.71 & 3.58 & 17 \\
\hline
\end{tabular}


Analysis 2.7. Comparison 2 SELECTIVE NORADRENALINE REUPTAKE INHIBITORS versus ACTIVE CONTROL, Outcome 7 Clinical global response: Average clinical global status score (CGI-S, high=worse) - short term (2 - 12 weeks).

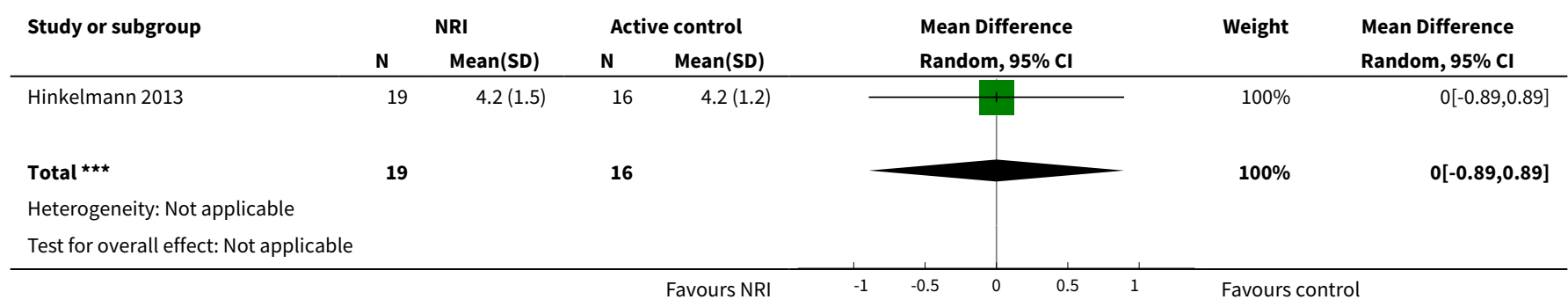

\section{Analysis 2.8. Comparison 2 SELECTIVE NORADRENALINE REUPTAKE INHIBITORS versus ACTIVE CONTROL, Outcome 8 Leaving the study early: 1a. Short term (2 - 12 weeks).}

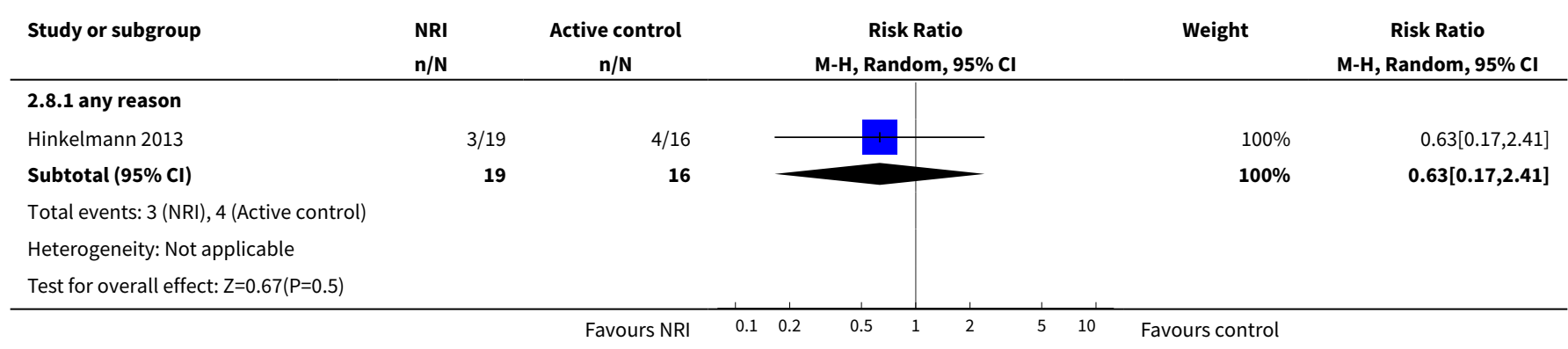

Analysis 2.9. Comparison 2 SELECTIVE NORADRENALINE REUPTAKE INHIBITORS versus ACTIVE CONTROL, Outcome 9 Leaving the study early: 1b. Medium term (13 - 26 weeks).

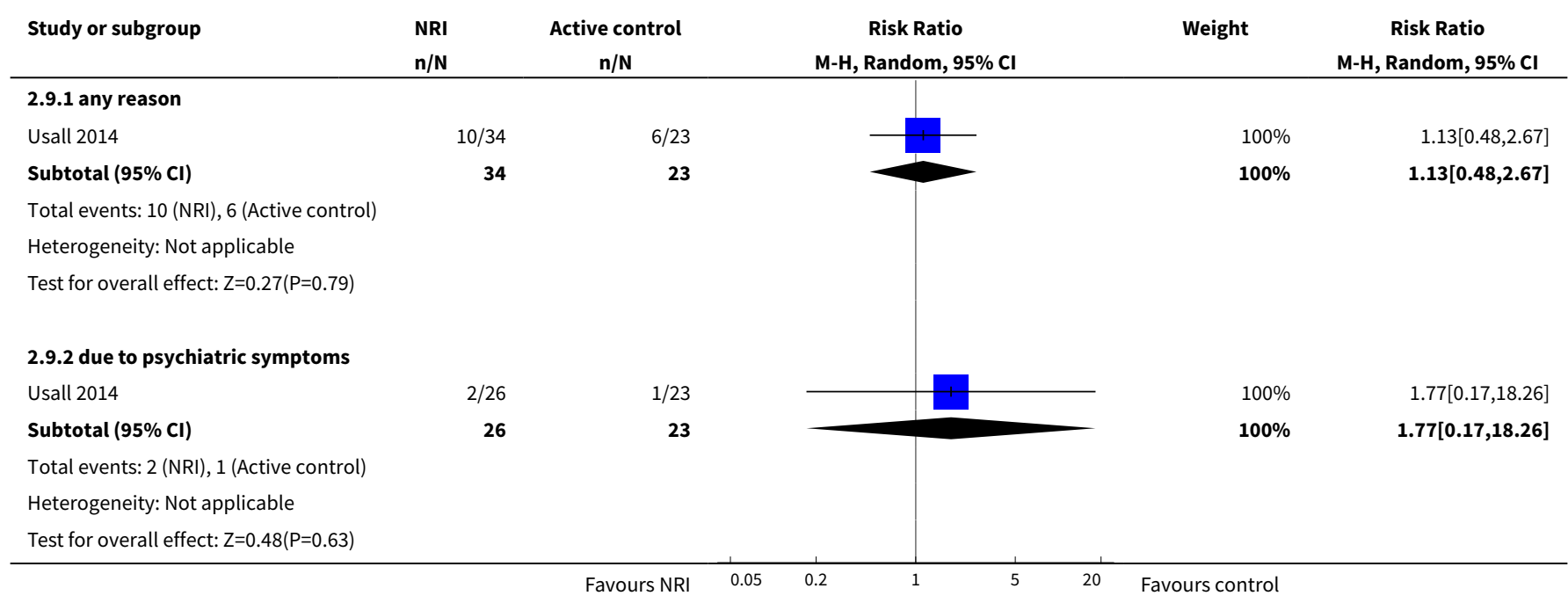

\section{ADDITIONAL TABLES}


Table 1. Mental state: 1.2 General - Average general/overall symptoms score (PANSS general, negative MD favours NRI) - short-term (2-12wks) - Subgroup and sensitivity analysis

\begin{tabular}{|c|c|c|c|c|c|}
\hline $\begin{array}{l}\text { Subgroup or sensitivity analysis } \\
\text { (see text for details) }\end{array}$ & $\begin{array}{l}\text { Number of } \\
\text { trials }\end{array}$ & $\mathbf{N}$ & $\begin{array}{l}\text { Mean differ- } \\
\text { ence }\end{array}$ & $\begin{array}{l}95 \% \text { Confidence in- } \\
\text { terval }\end{array}$ & $\mathbf{I}^{2}$ \\
\hline Overall outcome & 5 & 294 & -2.17 & -3.93 to $-0.40^{\star}$ & $44 \%$ \\
\hline Reboxetine & 4 & 279 & -2.20 & -4.15 to $-0.25^{\star}$ & $55 \%$ \\
\hline $\begin{array}{l}\text { Clozapine-only studies; excluding } \\
\text { skewed change-scores }\end{array}$ & 2 & 221 & -2.80 & -4.87 to $-0.72^{\star}$ & $75 \%$ \\
\hline $\begin{array}{l}\text { Excluding studies without clozapine; } \\
\text { negative symptoms }\end{array}$ & 3 & 256 & -2.40 & -4.48 to $-0.31^{\star}$ & $65 \%$ \\
\hline Excluding studies with imputed SD & 3 & 236 & -2.66 & -4.50 to $-0.82^{\star}$ & $55 \%$ \\
\hline Fixed-effect model & 5 & 294 & -2.73 & -3.71 to $-1.74^{\star}$ & $44 \%$ \\
\hline Low risk of randomisation bias & 2 & 58 & 0.89 & -3.60 to 5.37 & $0 \%$ \\
\hline
\end{tabular}

* Statistically significant at $\mathrm{p}<0.05$

Table 2. Mental state: 1.2 General - Average general/overall symptoms score (PANSS total, negative MD favours NRI) - short-term (2-12wks) - Subgroup and sensitivity analysis

\begin{tabular}{|c|c|c|c|c|c|}
\hline $\begin{array}{l}\text { Subgroup or sensitivity analysis } \\
\text { (see text for details) }\end{array}$ & $\begin{array}{l}\text { Number of } \\
\text { trials }\end{array}$ & $\mathbf{N}$ & $\begin{array}{l}\text { Mean differ- } \\
\text { ence }\end{array}$ & $\begin{array}{l}95 \% \text { Confidence in- } \\
\text { terval }\end{array}$ & $I^{2}$ \\
\hline Overall outcome & 4 & 309 & -2.84 & -5.28 to $-0.40^{\star}$ & $72 \%$ \\
\hline Clozapine-only studies & 2 & 220 & -4.25 & -5.89 to $-2.62^{\star}$ & $0 \%$ \\
\hline $\begin{array}{l}\text { Excluding outlier; excluding high risk } \\
\text { of bias }\end{array}$ & 3 & 244 & -4.20 & -5.82 to $-2.58^{*}$ & $0 \%$ \\
\hline $\begin{array}{l}\text { Negative symptoms; excluding im- } \\
\text { puted SD }\end{array}$ & 3 & 186 & -2.94 & -5.54 to $-0.34^{\star}$ & $81 \%$ \\
\hline Fixed-effect model & 4 & 309 & -2.12 & -3.12 to $-1.11^{*}$ & $72 \%$ \\
\hline
\end{tabular}

* Statistically significant at $p<0.05$

Table 3. Mental state: 1.2 General - Average general/overall symptoms score (PANSS total, negative MD favours NRI) - medium-term (13-26wks) - Exploration of heterogeneity

\begin{tabular}{|c|c|c|c|c|c|}
\hline $\begin{array}{l}\text { Subgroup or sensitivity analysis } \\
\text { (see text for details) }\end{array}$ & $\begin{array}{l}\text { Number of } \\
\text { trials }\end{array}$ & $\mathbf{N}$ & $\begin{array}{l}\text { Mean differ- } \\
\text { ence }\end{array}$ & $\begin{array}{l}95 \% \text { Confidence in- } \\
\text { terval }\end{array}$ & $I^{2}$ \\
\hline Overall outcome & 3 & 219 & -3.67 & -10.07 to 2.72 & $94 \%$ \\
\hline $\begin{array}{l}\text { Excluding outlier; excluding clozap- } \\
\text { ine study }\end{array}$ & 2 & 112 & -1.07 & -2.59 to 0.46 & $0 \%$ \\
\hline Fixed-effect model & 3 & 219 & -3.63 & -4.87 to $-2.39^{\star}$ & $94 \%$ \\
\hline
\end{tabular}


* Statistically significant at $p<0.05$

Table 4. Mental state: 1.5 Specific - Average negative symptoms score (PANSS negative, negative MD favours NRI) short-term (2-12wks) - Subgroup and sensitivity analysis

\begin{tabular}{|c|c|c|c|c|c|}
\hline $\begin{array}{l}\text { Subgroup or sensitivity analysis } \\
\text { (see text for details) }\end{array}$ & $\begin{array}{l}\text { Number of } \\
\text { trials }\end{array}$ & $\mathbf{N}$ & $\begin{array}{l}\text { Mean differ- } \\
\text { ence }\end{array}$ & $\begin{array}{l}95 \% \text { Confidence in- } \\
\text { terval }\end{array}$ & $I^{2}$ \\
\hline Overall outcome & 6 & 359 & -0.99 & -2.53 to 0.56 & $71 \%$ \\
\hline Reboxetine & 5 & 341 & -1.20 & -2.80 to 0.40 & $74 \%$ \\
\hline Clozapine-only studies & 2 & 221 & -1.60 & -3.96 to 0.76 & $84 \%$ \\
\hline Excluding studies without clozapine & 3 & 256 & -0.75 & -3.25 to 1.75 & $81 \%$ \\
\hline Negative symptoms & 4 & 321 & -1.47 & -3.04 to 0.09 & $77 \%$ \\
\hline Excluding imputed SD & 4 & 301 & -1.68 & -3.04 to $-0.32^{\star}$ & $70 \%$ \\
\hline Excluding skewed change scores & 3 & 286 & -1.92 & -3.28 to $-0.55^{\star}$ & $75 \%$ \\
\hline Fixed-effect model & 6 & 259 & -1.82 & -2.46 to $-1.18^{\star}$ & $71 \%$ \\
\hline Low risk of randomisation bias & 2 & 58 & 3.55 & -0.43 to 7.54 & $0 \%$ \\
\hline Excluding high risk of bias & 5 & 294 & -0.12 & -2.31 to 2.07 & $71 \%$ \\
\hline
\end{tabular}

* Statistically significant at $p<0.05$

Table 5. Mental state: 1.4 Specific - Average positive symptoms score (PANSS positive, negative MD favours NRI) short-term (2-12wks) - Subgroup and sensitivity analysis

\begin{tabular}{llllll}
\hline $\begin{array}{l}\text { Subgroup or sensitivity analysis } \\
\text { (see text for details) }\end{array}$ & $\begin{array}{l}\text { Number of } \\
\text { trials }\end{array}$ & $\mathbf{N}$ & $\begin{array}{l}\text { Mean differ- } \\
\text { ence }\end{array}$ & $\begin{array}{l}\text { 95\% Confidence in- } \\
\text { terval }\end{array}$ & $\mathbf{I}^{\mathbf{2}}$ \\
\hline Overall outcome & 5 & 294 & -0.16 & -0.96 to 0.63 & $0 \%$ \\
\hline Reboxetine & 4 & 279 & -0.24 & -1.05 to 0.57 & -1.02 to 0.72 \\
\hline Clozapine-only studies & 2 & 221 & -0.15 & -1.04 to 0.60 & $0 \%$ \\
\hline $\begin{array}{l}\text { Excluding studies without clozapine; } \\
\text { negative symptoms }\end{array}$ & 3 & 256 & -0.22 & -0.96 to 0.63 & $0 \%$ \\
\hline $\begin{array}{l}\text { Fixed-effect model } \\
\text { Low risk of randomisation bias }\end{array}$ & 2 & 294 & -0.16 & -2.93 to 1.40 & $0 \%$ \\
\hline
\end{tabular}

Table 6. Cognitive functioning: 2.3 Average composite cognitive functioning score (SMD, negative favours NRI) short-term (2-12wks) - Subgroup and sensitivity analysis

\begin{tabular}{|c|c|c|c|c|}
\hline $\begin{array}{l}\text { Subgroup or sensitivity analysis } \\
\text { (see text for details) }\end{array}$ & $\begin{array}{l}\text { Number of } \\
\text { trials }\end{array}$ & $\mathbf{N}$ & $\begin{array}{l}\text { Mean differ- } \\
\text { ence }\end{array}$ & $\begin{array}{l}95 \% \text { Confidence in- } \\
\text { terval }\end{array}$ \\
\hline
\end{tabular}


Table 6. Cognitive functioning: 2.3 Average composite cognitive functioning score (SMD, negative favours NRI) short-term (2-12wks) - Subgroup and sensitivity analysis (Continued)

\begin{tabular}{lccccc} 
Overall outcome & 4 & 180 & 0.04 & -0.28 to 0.36 & $8 \%$ \\
\hline Atomoxetine & 3 & 147 & -0.07 & -0.55 to 0.41 & $33 \%$ \\
\hline Fixed-effect model & 4 & 180 & 0.06 & -0.24 to 0.35 & $8 \%$ \\
\hline Low risk of randomisation bias & 2 & 55 & -0.18 & -1.06 to 0.69 & $61 \%$ \\
\hline Excluding high risk of other bias & 2 & 48 & 0.14 & -0.42 to 0.71 & -0.66 to 0.43 \\
\hline $\begin{array}{l}\text { Excluding high risk of attrition and re- } \\
\text { porting bias }\end{array}$ & 3 & 70 & & & $22 \%$ \\
\hline
\end{tabular}

Table 7. Cognitive functioning: 2.9 Average speed of processing score (SMD, negative favours NRI) - short-term (2-12wks) - Subgroup and sensitivity analysis

\begin{tabular}{|c|c|c|c|c|c|}
\hline $\begin{array}{l}\text { Subgroup or sensitivity analysis } \\
\text { (see text for details) }\end{array}$ & $\begin{array}{l}\text { Number of } \\
\text { trials }\end{array}$ & $\mathbf{N}$ & $\begin{array}{l}\text { Mean differ- } \\
\text { ence }\end{array}$ & $\begin{array}{l}95 \% \text { Confidence in- } \\
\text { terval }\end{array}$ & $I^{2}$ \\
\hline Overall outcome & 4 & 177 & 0.08 & -0.21 to 0.38 & $0 \%$ \\
\hline Atomoxetine & 3 & 144 & 0.04 & -0.29 to 0.37 & $0 \%$ \\
\hline Fixed-effect model & 4 & 177 & 0.08 & -0.21 to 0.38 & $0 \%$ \\
\hline Low risk of randomisation bias & 2 & 53 & 0.13 & -0.41 to 0.68 & $0 \%$ \\
\hline $\begin{array}{l}\text { Excluding high risk of attrition and } \\
\text { other bias }\end{array}$ & 2 & 48 & 0.23 & -0.34 to 0.80 & $0 \%$ \\
\hline Excluding high risk of reporting bias & 3 & 68 & 0.13 & -0.34 to 0.61 & $0 \%$ \\
\hline
\end{tabular}

Table 8. Cognitive functioning: 2.13 Average verbal learning/memory score (SMD, negative favours NRI) - shortterm (2-12wks) - Subgroup and sensitivity analysis

\begin{tabular}{|c|c|c|c|c|c|}
\hline $\begin{array}{l}\text { Subgroup or sensitivity analysis } \\
\text { (see text for details) }\end{array}$ & $\begin{array}{l}\text { Number of } \\
\text { trials }\end{array}$ & $\mathbf{N}$ & $\begin{array}{l}\text { Mean differ- } \\
\text { ence }\end{array}$ & $\begin{array}{l}95 \% \text { Confidence in- } \\
\text { terval }\end{array}$ & $\mathbf{I}^{2}$ \\
\hline Overall outcome & 4 & 181 & 0.01 & -0.31 to 0.32 & $7 \%$ \\
\hline Atomoxetine & 3 & 148 & -0.08 & -0.40 to 0.25 & $0 \%$ \\
\hline Fixed-effect model & 4 & 181 & 0.01 & -0.28 to 0.30 & $7 \%$ \\
\hline Low risk of randomisation bias & 2 & 55 & -0.06 & -1.04 to 0.93 & $69 \%$ \\
\hline Excluding high risk of other bias & 2 & 48 & 0.26 & -0.31 to 0.83 & $0 \%$ \\
\hline $\begin{array}{l}\text { Excluding high risk of attrition and re- } \\
\text { porting bias }\end{array}$ & 3 & 70 & -0.04 & -0.66 to 0.58 & $38 \%$ \\
\hline
\end{tabular}


Table 9. Clinical global response: 4.1 Average clinical global status score (CGI-S, negative MD favours NRI) - shortterm (2-12wks) - Subgroup and sensitivity analysis

\begin{tabular}{|c|c|c|c|c|c|}
\hline $\begin{array}{l}\text { Subgroup or sensitivity analysis (see } \\
\text { text for details) }\end{array}$ & $\begin{array}{l}\text { Number of } \\
\text { trials }\end{array}$ & $\mathbf{N}$ & $\begin{array}{l}\text { Mean differ- } \\
\text { ence }\end{array}$ & $\begin{array}{l}95 \% \text { Confidence } \\
\text { interval }\end{array}$ & $I^{2}$ \\
\hline Overall outcome & 5 & 160 & -0.03 & -0.35 to 0.28 & $7 \%$ \\
\hline Reboxetine; excluding high risk of bias & 4 & 137 & 0.10 & -0.23 to 0.42 & $0 \%$ \\
\hline Fixed-effect model & 5 & 160 & -0.03 & -0.32 to 0.27 & $7 \%$ \\
\hline
\end{tabular}

Table 10. Adverse effects: 6.2.1 All cause withdrawals (lower RR favours NRI) - short-term (2-12wks) - Subgroup and sensitivity analysis

\begin{tabular}{llllll}
\hline $\begin{array}{l}\text { Subgroup or sensitivity analysis } \\
\text { (see text for details) }\end{array}$ & $\begin{array}{l}\text { Number of } \\
\text { trials }\end{array}$ & $\mathbf{N}$ & Relative risk & $\begin{array}{l}\text { 95\% Confidence in- } \\
\text { terval }\end{array}$ & $\mathbf{I}^{\mathbf{2}}$ \\
\hline Overall outcome & 8 & 401 & 0.94 & 0.63 to 1.39 & $0 \%$ \\
\hline Atomoxetine & 3 & 173 & 0.89 & 0.49 to 1.59 & 0.54 to 1.70 \\
\hline Reboxetine & 5 & 200 & 0.95 & 0.64 to 1.41 & $0 \%$ \\
\hline Fixed-effect model & 8 & 401 & 0.95 & 0.54 to 1.56 & $0 \%$ \\
\hline Low risk of bias in randomisation & 5 & 182 & 1.03 & 0.62 to 1.70 & $0 \%$ \\
\hline Excluding high risk of other bias & 7 & 248 & 0.97 & 0.58 to 1.60 & $0 \%$ \\
\hline Excluding high risk of reporting bias & 7 & 252 & 369 & 0.96 & 0.63 to 1.45 \\
\hline Excluding high risk of attrition bias & 8 & & & $0 \%$ \\
\hline
\end{tabular}

Table 11. Adverse effects: 6.2.2 Withdrawal due to adverse events (lower RR favours NRI) - short-term (2-12wks) Subgroup and sensitivity analysis

\begin{tabular}{|c|c|c|c|c|c|}
\hline $\begin{array}{l}\text { Subgroup or sensitivity analysis } \\
\text { (see text for details) }\end{array}$ & $\begin{array}{l}\text { Number of } \\
\text { trials }\end{array}$ & $\mathbf{N}$ & Relative risk & $\begin{array}{l}95 \% \text { Confidence in- } \\
\text { terval }\end{array}$ & $\mathbf{I}^{2}$ \\
\hline Overall outcome & 4 & 178 & 2.08 & 0.70 to 6.21 & $0 \%$ \\
\hline Atomoxetine & 3 & 153 & 1.88 & 0.58 to 6.08 & $0 \%$ \\
\hline Fixed-effect model & 4 & 178 & 2.18 & 0.79 to 6.02 & $0 \%$ \\
\hline Low risk of bias in randomisation & 2 & 53 & 1.15 & 0.15 to 8.60 & $18 \%$ \\
\hline Excluding high risk of other bias & 2 & 45 & 4.48 & 0.56 to 35.52 & $0 \%$ \\
\hline
\end{tabular}


Table 11. Adverse effects: 6.2.2 Withdrawal due to adverse events (lower RR favours NRI) - short-term (2-12wks) Subgroup and sensitivity analysis (Continued)
Excluding high risk of reporting bias
3
73
1.66
0.36 to 7.72
$0 \%$

Table 12. Included and excluded studies and relevant Cochrane Reviews

\begin{tabular}{|c|c|c|c|c|}
\hline Study tag & $\begin{array}{l}\text { Specific patient charac- } \\
\text { teristics }\end{array}$ & Intervention & Control & Relevant Cochrane Reviews \\
\hline Barnes 2009 & Negative symptoms & Citalopram & Placebo & Rummel-Kluge 2006 \\
\hline Hou 2007 & Negative symptoms & Citalopram & Placebo & Rummel-Kluge 2006 \\
\hline Mueller 2005 & & Celecoxib & Placebo & Akhondzadeh 2011 \\
\hline Salokangas 1997 & & Citalopram & Placebo & - \\
\hline \multirow[t]{6}{*}{ Shafti 2004} & & Clomipramine & Placebo & - \\
\hline & & Citalopram & & - \\
\hline & & Fluoxetine & Placebo & - \\
\hline & & Nortiptyline & & - \\
\hline & & Fluvoxamine & Placebo & - \\
\hline & & Maprotiline & & - \\
\hline Hinkelmann 2013 & Negative symptoms & Reboxetine & Placebo & Rummel-Kluge 2006 \\
\hline Li 2008 & Negative symptoms & Reboxetine & Placebo & Rummel-Kluge 2006 \\
\hline Shafti 2015 & Negative symptoms & Reboxetine & Placebo & Rummel-Kluge 2006 \\
\hline Usall 2014 & Negative symptoms & Reboxetine & Placebo & Rummel-Kluge 2006 \\
\hline Yu 2012 & Negative symptoms & Reboxetine & Control & Rummel-Kluge 2006 \\
\hline Zhao 2013 & Negative symptoms & Reboxetine & Placebo & Rummel-Kluge 2006 \\
\hline
\end{tabular}

\section{Table 13. Suggested design for a future NRI trial}

$\begin{array}{ll}\text { Methods } & \text { Allocation: randomised (with sequence generation and allocation concealment clearly described) } \\ \text { Blinding: double blind (participants, clinicians, and outcome assessors) and blinding tested } \\ \text { Duration: at least } 3 \text { months } \\ \text { Design: parallel group } \\ \text { Setting: inpatient and outpatient } \\ \text { Participants } & \text { Diagnosis: people with schizophrenia (duration }>6 \mathrm{~m} \text { ) and prominent negative symptoms (e.g. } \\ \text { PANSS negative }>30) \\ \mathrm{N}=300\end{array}$


Table 13. Suggested design for a future NRI trial (Continued)

Age: Any, but with recruitment focused on those aged 18-65yrs

Sex: Men and women

Interventions

1. Reboxetine (8-12mg daily) in combination with any antipsychotic treatment including clozapine

2. Placebo in combination with any antipsychotic treatment including clozapine

Outcomes

Mental state: significant improvement in negative symptoms defined as $20 \%$ reduction in the PANSS negative scale*, average scores on the PANSS negative scale, significant improvement and average scores on the PANSS total, general, and positive scales and the HRSD

Cognitive functioning: significant improvement in a clinical scale of cognitive functioning such as the Schizophrenia Cognition Rating Scale (SCORS) total score, average scores on neurocognitive testing such as the MATRICS Consensus Cognitive Battery (MCCB) overall composite T-score and individual domain scores

Quality of life: significant improvement in a quality of life scale, average scores on a quality of life scale

Clinical global response: significant improvment in clinical global status defined as CGI-I scores of 1 or 2, average scores on the CGI-S

Service utilisation

Adverse effects: leaving the study early (for any reason), serious adverse effects, average weight gain

Satisfaction with treatment

Social or general functioning

* recommended primary outcome, other outcomes listed are suggestions rather than an exhaustive list

\section{AP PE N D I C E S}

\section{Appendix 1. Previous Searches}

\section{November 2012}

\section{Electronic searches}

\section{Cochrane Schizophrenia Group Trials Register (November 2012)}

We searched the register using the phrase:

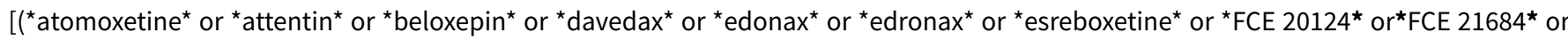

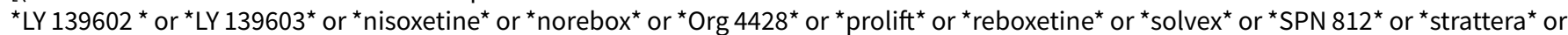
${ }^{\star}$ talopram ${ }^{\star}$ or ${ }^{\star}$ talsupram ${ }^{\star}$ or ${ }^{\star}$ tomoxetin ${ }^{\star}$ or ${ }^{\star}$ vestra ${ }^{\star}$ or ${ }^{\star} \mathrm{NRI}^{\star}$ or ${ }^{\star}$ noradrenaline reuptake ${ }^{\star}$ or ${ }^{\star}$ norepinephrine reuptake ${ }^{\star}$ in interventions of STUDY) or ("norepinephrine reuptake* or *noradrenaline reuptake* in title, abstract or index terms of REFERENCE)]

This register is compiled by systematic searches of major databases, handsearches and conference proceedings (see group module).

\section{Searching other resources}

\section{Reference searching}

We will inspect references of all identified studies for further relevant studies.

\section{Personal contact}

We will contact the first author of each included study for information regarding unpublished trials. 


\section{H IS T O R Y}

Protocol first published: Issue 11, 2012

Review first published: Issue 1, 2018

\begin{tabular}{lll}
\hline Date & Event & Description \\
\hline 17 November 2015 & Amended & $\begin{array}{l}\text { Search was updated and 4 studies were added to 'Classification } \\
\text { pending references' section of the review. }\end{array}$ \\
\hline 20 August 2014 & Amended & $\begin{array}{l}\text { Search was updated and six new references were added to 'Clas- } \\
\text { sification pending references' section of the the review. }\end{array}$ \\
& $\begin{array}{l}\text { Note: References from previous search are still in 'Studies await- } \\
\text { ing classification' section of the review. }\end{array}$
\end{tabular}

\section{CONTRIBUTIONS OF AUTHORS}

Paul Matthews: screening retrieved papers against eligibility criteria, appraising the quality of papers, extracting data, contacting authors for additional information, handsearching references, calculating additional useable data from reported values, entering data into RevMan 5, analysis of data, interpretation of data, writing the protocol and review, liaison with the Cochrane Schizophrenia Group.

Mike Pearce: appraising the quality of papers, extracting data, interpretation of data, writing the review.

Jamie Horder: screening retrieved papers against eligibility criteria, writing the protocol and review.

\section{DECLARATIONS OF INTEREST}

Paul Matthews: had a short clinical attachment to the Schizophrenia Research Program at NIMH in 2009 during the period that Apud 2007a was conducted there, but did not have direct involvement in the trial. From 2011 to 2016 PM has attended a total of two days of educational meetings organised by Lundbeck and one day organised by Eli Lilly in the UK and received travel expenses on two of these occasions.

Jamie Horder: none known.

Michael Pearce: none known.

\section{SOURCES OF SUPPORT}

\section{Internal sources}

- Oxford Health NHS Foundation Trust, Oxford, UK.

Previously employed lead author Paul RL Matthews and employs review author Michael Pearce.

- King's College London, UK.

Employs review author Jamie Horder.

- Berkshire Healthcare NHS Foundation Trust (Bracknell), UK.

Previously employed review authors Paul Matthews and Michael Pearce.

\section{External sources}

- No sources of support supplied

\section{DIFFERENCES BETWEEN PROTOCOLANDREVIEW}

The protocol for this review was published as Matthews 2012.

\section{Inclusion criteria}

We decided to broaden the inclusion criteria of the review beyond placebo-controlled trials to better reflect the title of the review and identified two trials using citalopram as an active comparator that were also included in the placebo-controlled analysis. 


\section{Search methods}

After discussion with the Cochrane Schizophrenia Group TSC we broadened the search terms in order to include more potential selective NRIs. One additional study was identified utilising viloxazine.

In the protocol we stated that we would contact the first author of each study but due to the practicalities involved we made the pragmatic decision to contact the corresponding author for each study instead as contact details were more readily accessible and we considered they would be more likely to have relevant data available.

\section{Data collection and analysis}

We specified in the protocol that PM and JH would extract data and analyse risk of bias but PM and MP carried out this role when MP joined the final review.

In the protocol we stated that we would use the equation from the Cochrane manual for relating baseline, change, and endpoint SD in order to impute the SD for endpoint data where we only have baseline and change scores. However, this was mathematically underdetermined and we instead substituted the baseline SD as advised in the Cochrane Handbook for Systematic Reviews of Interventions (Higgins 2011).

We continued to utilise the assumption of a 0.5 correlation between baseline and endpoint scores to estimate the SD of change scores as outlined in the protocol but we were unable to validate this assumption by deriving empirical estimates of the correlation from other studies as none reported all the relevant values.

It was not clear in the original protocol that we would analyse outcomes based on the duration of the trial as we had also listed these under subgroup analyses, so we clarified this in the Methods and specified how to handle multiple outcomes within the same duration.

We made the post hoc decision to perform subgroup and sensitivity analyses for secondary outcomes as well as for the primary outcomes. This was because there was limited data available for our primary outcome measures and interpretation of secondary outcomes of significant interest (e.g. negative symptom scores) was difficult without this.

In the original protocol we had specified that we would only produce a meta-analytic outcome when there were at least three trials available to combine; however this restriction is the not the usual practice of the Cochrane Schizophrenia Group and after discussions we agreed to remove this requirement. As a consequence of this decision we added the clarification that subgroup and sensitivity analyses would only be conducted when there were more than three studies as sensitivity analysis is not meaningful if there are only one or two trials.

Although we could not determine whether change scores were skewed we were often able to calculate endpoint scores from these and to estimate the SD which frequently suggested skew. While using the change scores rather than endpoint scores could correct this skew it was not possible to test this so we made a post hoc decision to perform a sensitivity analysis where we excluded those change scores which were associated with skewed endpoint scores.

In the protocol we stated that we would produce a 'Summary of findings' table listing an important specific adverse effect but did not define what this adverse effect would be. Prior to performing the analysis of adverse effects, we selected 'nausea' based on the most common adverse effects listed for reboxetine and atomoxetine in the British National Formulary (BNF 2016).

As we had not specified in advance the duration of studies to include in the 'Summary of findings' table we made a post hoc decision to use the analysis with the longest period of follow-up providing there were more than three studies to include for that time period; otherwise we used the duration which included the most studies. Where there was more than one outcome measure for a time point, we used the one with the most studies.

\section{N DEX TERMS}

\section{Medical Subject Headings (MeSH)}

Adrenergic Uptake Inhibitors [ ${ }^{*}$ therapeutic use]; Atomoxetine Hydrochloride [therapeutic use]; Citalopram [therapeutic use]; Cognition [drug effects]; Morpholines [therapeutic use]; Quality of Life; Randomized Controlled Trials as Topic; Reboxetine; Schizophrenia [ ${ }^{*} \mathrm{drug}$ therapy]; Serotonin and Noradrenaline Reuptake Inhibitors [ ${ }^{*}$ therapeutic use]; Viloxazine [therapeutic use]

\section{MeSH check words}

Adult; Humans 